\title{
Replicating DNA at top speed : chromatin at risk
}

Citation for published version (APA):

Spaapen, F. A. (2010). Replicating DNA at top speed : chromatin at risk. [Doctoral Thesis, Maastricht University]. Maastricht University. https://doi.org/10.26481/dis.20100305fs

Document status and date:

Published: 01/01/2010

DOI:

$10.26481 /$ dis.20100305fs

Document Version:

Publisher's PDF, also known as Version of record

\section{Please check the document version of this publication:}

- A submitted manuscript is the version of the article upon submission and before peer-review. There can be important differences between the submitted version and the official published version of record.

People interested in the research are advised to contact the author for the final version of the publication, or visit the DOI to the publisher's website.

- The final author version and the galley proof are versions of the publication after peer review.

- The final published version features the final layout of the paper including the volume, issue and page numbers.

Link to publication

\footnotetext{
General rights rights.

- You may freely distribute the URL identifying the publication in the public portal. please follow below link for the End User Agreement:

www.umlib.nl/taverne-license

Take down policy

If you believe that this document breaches copyright please contact us at:

repository@maastrichtuniversity.nl

providing details and we will investigate your claim.
}

Copyright and moral rights for the publications made accessible in the public portal are retained by the authors and/or other copyright owners and it is a condition of accessing publications that users recognise and abide by the legal requirements associated with these

- Users may download and print one copy of any publication from the public portal for the purpose of private study or research.

- You may not further distribute the material or use it for any profit-making activity or commercial gain

If the publication is distributed under the terms of Article $25 \mathrm{fa}$ of the Dutch Copyright Act, indicated by the "Taverne" license above, 
Replicating DNA at Top Speed

Chromatin at Risk 
Printed by GVO printers \& designers B.V. | Ponsen \& Looijen ISBN 978-90-9025128-8

(C) Frank Spaapen, Maastricht 2010 


\title{
Replicating DNA at Top Speed
}

\section{Chromatin at Risk}

\author{
PROEFSCHRIFT \\ ter verkrijging van de graad van doctor \\ aan de Universiteit Maastricht, \\ op gezag van de Rector Magnificus, \\ Prof. mr. G.P.M.F. Mols \\ volgens het besluit van het College van Decanen, \\ in het openbaar te verdedigen \\ op vrijdag 5 maart 2010 om 14.00 uur \\ door
}

Franciscus Alfonsus Spaapen

Geboren te Haarlem op 7 februari 1978 
Promotor:

Prof. dr. J.P.M. Geraedts

Copromotor:

Dr. J.W. Voncken

Beoordelingscommissie:

Prof. dr. F.C.S. Ramaekers (voorzitter)

Prof. dr. C.M. Verfaillie (KU-Leuven)

Dr. J. Robben (KU-Leuven)

Prof.dr. J. Kleinjans

Dr. R.C.J. Langen

This research was supported by

NWO

Reumafonds.

Printing of this thesis was financially supported by Greiner Bio-one.

greinerbio-one 


\section{Contents}

$\begin{array}{ll}\text { Chapter } 1 & 7\end{array}$

$\begin{array}{ll}\text { General Introduction } & 11 \\ \text { Aim of the thesis } & 11\end{array}$

Chapter 2

Replicating DNA at Top Speed - Chromatin at Risk. (New tales from aging bones).

Chapter 3

A novel in vivo model to study enchondral bone formation; HIF-1 $\alpha$ activation and BMP expression

\section{Chapter 4}

The immediate early gene EGR1 controls epigenetic reprogramming through Polycomb Group proteins during chondrogenesis.

\section{Chapter 5}

Polycomb Repressive Complex 1 Coordinates Enhanced Replication and Transcription in Transit Amplifying Chondrogenic Progenitors.

\section{Chapter 6}

Proliferative Capacity Defines Sensitivity to Valproic Acid During Chondrogenesis.

Chapter 7

General Discussion

\section{Summary}

Samenvatting

Dankwoord 



\section{Chapter 1}

\section{General introduction}


Chapter 1

\section{Introduction}

DNA stores all genetic information that is required for a cell to manufacture the molecules it needs to function. Although most somatic cells in a human body contain identical DNA sequences, the vast variety of cellular phenotypes implies that the information stored in the DNA sequence on its own is not sufficient to explain cell diversity and function. Cells depend on the acquisition and the maintenance of epigenetic traits to acquire and maintain a specific phenotype. An epigenetic trait is formally defined as "a stably heritable phenotype resulting from changes in a chromosome without alterations in the DNA sequence" " . Genomic DNA is a major constituent of chromatin, a complex structure consisting of DNA, and all associated proteins and RNA. The nucleosome is the fundamental unit of chromatin, consisting of two copies of the 4 core histones $(\mathrm{H} 2 \mathrm{~A}, \mathrm{H} 2 \mathrm{~B}, \mathrm{H} 3$ and H4) with 147 base pairs of DNA wrapped around it. N-terminal histone 'tails' protrude from chromatin and are subject to a wide variety of posttranslational modifications. Over recent years, many enzymes and chromatin associated proteins have been identified that can write, erase and/or read these modifications, providing an epigenetic register of, for instance, transcriptional activity. Increasing evidence suggests that epigenetic processes dynamically regulate partitioning of the genome in 'active' and 'inactive' chromatin. Chromatin can undergo dynamic changes in structure, resulting in various states of compaction, which either facilitate or block accessibility of the DNA template. Chromatin structure modification is an important regulatory aspect of all DNA templated processes, including e.g. transcription, replication and repair ${ }^{2}$.

\section{Epigenetics}

Increasing insights into the molecular mechanisms underlying epigenetic regulation reveals an incredibly complex apparatus that has major implications for development and disease. Although epigenetic regulation controls several processes, regulation of transcription is understood best, and this occurs at the level of RNA, DNA and proteins.

At the DNA level, cytosines in $\mathrm{CpG}$ dinucleotides were found to be methylated by DNA methyltransferases (DNMTs) and promoter DNA methylation by and large correlates with transcriptional silencing ${ }^{3}$.

At the RNA level, non-coding RNAs were identified as transcriptional repressors ${ }^{4}$. Perhaps the best known non-coding RNA is Xist: Xist covers the outside of the inactive X-chromosome and thereby maintains the transcriptionally inactive state ${ }^{5}$. Another mechanism by which non-coding RNAs silence gene expression is RNA interference (RNAi): microRNA's and small interfering RNA's (siRNAs) bind RNA through (sometimes imperfect) sequence homology thereby regulate gene expression through blocking gene transcription, regulating RNA translation, RNA processing or RNA stability ${ }^{4}$. For example, non-coding RNA can bind messenger RNA (mRNA) to prevent translation or to activate the RNAi pathway to degrade mRNAs by recruiting the Risc complex to mRNAs. Interestingly, binding of non-coding RNAs to specific mRNAS was also shown to enhance translation. 
At the protein level, N-terminal histone tails in nucleosomes are subject to numerous posttranslational modifications (i.a. (mono-, di-, or tri-) methylation, phosphorylation, acetylation, ubiquitylation) by a diversity of enzymes, such as histone methyltransferases (HMTs), kinases, histone acyltransferases (HATs) and ubiquitylases ${ }^{2}$. Other enzymes are specialized in removing histone modifications, e.g. histone demethylases (HDMs), phosphatases, histone deacetylases (HDACs) and deubiquitylases ${ }^{2}$. Proteins can recognize and bind to specific modifications through specialized domains, forming chromatin-associated multi-protein complexes with relevant epigenetic activity. In addition to the various types of modifications that can occur, histones can be modified at multiple sites and may be recognized in combination. In the context of transcription, these collective histone modifications constitute an epigenetic index of gene activity. How epigenetics precisely regulates gene activity is incompletely understood. Certain modifications strongly correlate to transcriptional activity, or inactivity, although no perfect correlation has been found to date. Together, this array of writers, erasers and binders of epigenetic modifications suggests complex and dynamic epigenetic regulation of gene activity.

\section{Polycomb Group proteins}

Polycomb Group (PcG) proteins are best known as transcriptional repressors. First identified in Drosophila melanogaster as important regulators of segmentation, PcG complexes maintain expression boundaries of homeobox (Hox) genes within the Homeotic gene clusters (HOM-C) along the antero-posterior (AP) axis. Hox genes are important regulators of segment identity in the fly. It is clear that PcG proteins exert a repressive function and that PcG proteins and their targets are conserved throughout evolution. Also in mammals PcG control segmentation: Hox genes determine vertebral identity along the AP-axis. PcG-mutations in the mouse cause vertebral identity changes and other skeletal defects ${ }^{6}$. PcG proteins form large multiprotein complexes that combine intrinsic with associated epigenetic activities. PcG repressive complex 2 (PRC2) for instance harbors Eed, SUZ12 and the HMT EZH2, which harbors a SET domain with HMT activity for lysine 27 on histone 3 (Figure 1). This PcG associated posttranslational modification is recognized and bound by specific chromobox proteins (CBX) in the PRC1 complex that also contains PHC, BMI1, RING1 and RNF2 (Figure 1). In addition the PRC1 complex harbors E3 ligase activity for lysine 119 of $\mathrm{H} 2 \mathrm{~A}$. Both PRCs are associated with many other factors relevant for regulation of transcription. In the fruit fly larvae PRC2 is believed to initiate PcG-based gene silencing, whereas PRC1 takes over as a maintenance complex. Although PcG are known as transcriptional repressors, how PRCs control repression of promoter activity is mechanistically far from understood. 


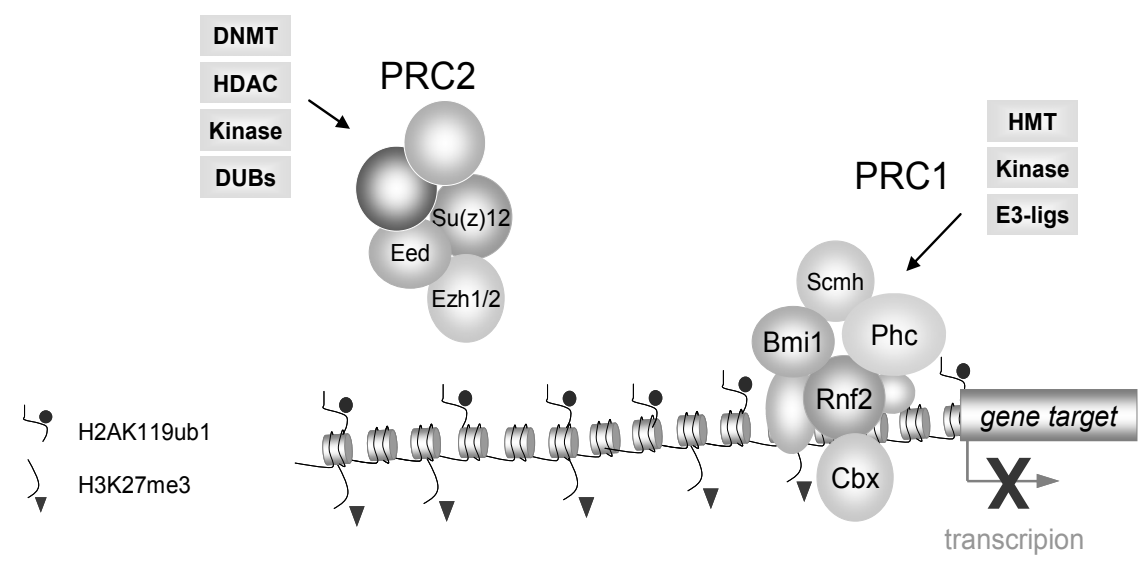

Figure 1 Schematic overview PRC1 and PRC2 complexes

\section{Enchondral ossification}

Enchondral ossification is a multistep process in which the ossified skeleton is formed from a cartilaginous matrix. The local microenvironment plays an important role in regulation of the different steps of Enchondral ossification. Locally produced soluble factors, such as insulin-like growth factors, bone morphogenetic proteins, fibroblast growth factors and many others ${ }^{7}$ regulate the different stages of chondrocyte and osteocytes maturation.

Cartilage is a dense connective tissue, consisting of collagen, proteoglycan and elastin fibres, excreted by chondrocytes. Cartilage is characterized by the absence of blood vessels, and thus the hypoxic environment. Therefore chondrocytes receive nutrients and oxygen by diffusion from synovial fluids, and depend heavily on hypoxia-inducible factor 1 (HIF-1) and anaerobic metabolism to function and survive ${ }^{7}$.

Longitudinal growth of bones is accomplished by two growth plates (epiphysary regions) that move away from each other as condensations of mesenchymal (stem/progenitor) cells differentiate into chondrocytes. The growth plates are arranged in distinct regions that reflect the different stages of chondrocyte differentiation. The first phase of chondrocyte differentiation is characterized by activation of the proliferative capacity of resting chondrocytes and longitudinal growth occurs. Next, the proliferating chondrocytes become flattened and packed in condensed clusters. Through a phase of pre-hypertrophy, the chondrocytes terminally differentiate into hypertrophic chondrocytes. In addition to excretion of the cartilage matrix, hypertrophic chondrocytes secrete i.a. VEGF to induce the formation of blood vessels, and TGF $\beta$ to attract mesenchymal cells and to differentiate these cells into osteocytes. As blood vessels and osteocytes enter the cartilage matrix, chondrocytes undergo programmed cell death and osteocytes calcify the extracellular matrix to form bone. 


\section{Aim and outline of this thesis}

Mechanisms underlying PcG mediated transcriptional repression are beginning to unravel. In addition, although poorly understood, PcG proteins have been associated with replication and DNA repair. However, many questions about Polycomb group biology remain: how are PcG proteins regulated themselves? How are PcG proteins involved in mediating gene-environment interactions? How are PCG proteins targeted to cell-type specific targets? What is the mechanism behind PcG mediated transcriptional repression? What is the role of PcG in replication and DNA repair? What is the role of PcG in enchondral ossification?

We here specifically focus on the role of PcG proteins in chondrogenesis. PcG mutant mice display skeletal malformations along the AP-axis, associated with deregulation of Hox gene expression boundaries. However, very little is known about the direct involvement of PcG proteins in enchondral ossification. The working hypothesis of the research described in this thesis is that PcG proteins mediate gene-environment interactions, thereby allowing the epigenomic landscape to change and to facilitate chondrogenic differentiation. The research described in this research aimed to elucidate the role of PcG proteins and underlying molecular mechanisms in chondrogenic differentiation.

In chapter 2, a detailed review of our current understanding of epigenetic regulation of transcription and replication, with a particular focus on PcG proteins, is presented. Chapter 3 describes a novel in vivo model to study enchondral ossification in relation to gene-environment interactions, with important relevance to alternative tissue repair strategies in regenerative medicine. In chapter 4, Egr1 was identified as an important immediate early transcription factor, rapidly induced upon chondrogenic differentiation. We find that Egr1 directly controls epigenetic remodelling required for chondrogenesis, and present evidence for interaction with PcG function at several levels. Chapter 5 describes the role for Bmi1 in regulating transcription and replication during transit amplification of differentiating chondrocytes. In chapter 6, we show that the epigenetic drug valproic acid induces replication stress during transit amplifying chondrocytes, leaving the cells incapable of fulfilling the differentiation program. Finally, in chapter 7 the results obtained in these studies are discussed. 
Chapter 1

\section{References}

$1 \quad$ Berger, S. L., Kouzarides, T., Shiekhattar, R., and Shilatifard, A., Genes Dev 23 (7), 781 (2009).

$2 \quad$ Kouzarides, T., Cell 128 (4), 693 (2007).

3 Rountree, M. R., Bachman, K. E., Herman, J. G., and Baylin, S. B., Oncogene 20 (24), 3156 (2001).

$4 \quad$ Nowotny, M. and Yang, W., Curr Opin Struct Biol 19 (3), 286 (2009).

$5 \quad$ Chow, J. and Heard, E., Curr Opin Cell Biol 21 (3), 359 (2009).

$6 \quad$ Alkema, M. J. et al., Nature 374 (6524), 724 (1995); del Mar, L. M. et al., Development 127 (23), $5093(2000)$.

7 Mackie, E. J. et al., Int J Biochem Cell Biol 40 (1), 46 (2008). 


\title{
Chapter 2
}

\section{Replicating DNA at Top Speed \\ - Chromatin at Risk.}

(New tales from aging bones).

\author{
F. Spaapen, Y. Takihara, H. Brock, J.W. Voncken
}




\begin{abstract}
Polycomb Group proteins have been associated with numerous cellular processes that use chromatin as a template, including transcription, replication and DNA repair. The link between Polycomb function and DNA replication is a long-standing one, yet poorly understood. Recent observations position Polycomb proteins at the cross road of transcription and replication and begin to offer explanations for cellular and morphological phenotypes. Here we review this emerging role for Polycomb in the context of development and cancer.
\end{abstract}

\title{
Introduction
}

Lineage commitment and terminal differentiation in the context of development are dependent on acquisition and maintenance of heritable epigenetic traits. An epigenetic trait is formally defined as "a stably heritable phenotype resulting from changes in a chromosome without alterations in the DNA sequence" 2 . Although gradually unfolding, the epigenetic mechanisms underlying stable inheritance of cell fate decisions (i.e. in mitosis and meiosis) are still far from being completely understood. Chromatin remodeling underlies all DNA-templated cellular processes such as replication, transcription and repair. Continuous redefinition of chromatin states by covalent modification of DNA (i.e. CpG methylation) and/or chromatin associated proteins (e.g. posttranslational modifications of core histones), allows a cell to undergo cell fate determination and to function in its micro-environment, i.e. to respond to constantly changing physiological or metabolic conditions. Lineage commitment and early differentiation are likely to inflict massive strain on the genome, which, under these conditions, is simultaneously transcriptionally redefined and replicated. Both the transcriptome and the genome are subject to dynamic changes throughout differentiation. Within the context of differentiation, however, the underlying epigenetic mechanisms that help tuning replication to transcription and vice versa remain poorly understood. It is conceivable that differential epigenomic states functionally partition the genome and help guide cells through differentiation, establish and maintain novel physiological states, while allowing genome duplication without compromising genetic integrity and genome stability. Thus DNA transcription, DNA replication and DNA repair require coordination if they are to occur from one and the same template. Perhaps, predictably, transcription, replication and repair 'machineries' share basal components. It is likely that such proteins and the regulatory pathways they control evolved to tune these processes in the context of differentiation in multi-cellular organisms. Polycomb Group proteins are connected to many processes that require chromatin structure remodeling, among which transcription, replication and repair ${ }^{3,4}$. Although biochemical processes relevant to epigenetic regulation of transcriptional repression by $P_{c G}$ complexes are becoming increasingly clear, how exactly cellular memory is installed and whether and how this connects to DNA replication remain enigmatic. We here review how specialized pathways and chromatin structure control help cells cope with several important chromatin-based processes (like 
transcription and replication) that, although seemingly in conflict with each other, often occur simultaneously in the context of differentiation and development. Specific focus will be on the potential role of Polycomb Group (PcG) proteins in these control mechanism.

\section{Epigenetics in transcription}

Perhaps the most studied and mechanistically relatively best understood example of epigenetic control of a DNA-templated process is that of transcription. Transcription, production of RNA from DNA, is a process highly coordinated by a variety of proteins surrounding the key factor in transcription: RNA polymerase. Polyadenylated RNA synthesis requires formation of a preinitiation complex (PIC) by RNA polymerase II (RPOL II) with the general transcription factors (GTF) TFIID, TFIIA and TFIIB ${ }^{5}$. GTF direct RPOLII to these core elements and transcription is initiated by ATPdependent TFIIH helicase activity, which uncoils and denatures the DNA allowing RPOLII to bind single stranded DNA. To date, in eukaryotes seven core promoter elements have been identified (e.g. TATA box), which comprise specific DNA sequences required for promoter function and for proper assembly of the PIC ${ }^{6}$. Both initiation of transcription and elongation of RNA by RPOLII are phosphorylation dependent events ${ }^{7,8}$. Access to DNA by local chromatin structure remodelling events is essential for initiation and elongation. This is controlled at the epigenetic level and includes both covalent histone modifications and nucleosome remodeling machineries ${ }^{9}$. Although the order of these events is currently under debate, they essentially recruit transcriptional activators and expose DNA binding elements.

Select post-translational modifications (PTM) of histone proteins are linked to specific biological processes. Examples include histone variant $\mathrm{H} 2 \mathrm{AX}$ phosphorylation in the context of DNA damage responses and specific histone $\mathrm{H} 3$ acetylation and methylation events that are often associated with activation and repression of transcription, respectively ${ }^{3,7,10}$. Histone modifications often occur and function in conjunction, for example, histone acetylation and methylation events are collectively responsible for nucleosome displacement and transcriptional activation ${ }^{11}$. Overall, the epigenome is defined by a combination of molecular mechanisms at all three macromolecular levels, DNA, RNA and protein, which are thought to collectively constitute a highly complex register of gene status ${ }^{4}$. Some modifications occur in correlation with higher-order chromatin structures. For example, as opposed to euchromatin, condensed constitutive heterochromatin is often associated with $\mathrm{H} 3 \mathrm{~K} 9 \mathrm{me} 3$. Compared to euchromatin, heterochromatin often displays distinct subnuclear localisation, is replicated later during S-phase and heterochromatic DNA repair requires additional components ${ }^{12}$. Although a putative inductive role for H3K9me3 in chromatin condensation is neither proven nor clear, the correlation does illustrate an important concept: the eukaryotic genome is functionally partitioned by the epigenome. 


\section{Polycomb in Transcription}

Polycomb Group (PcG) proteins are best known as transcriptional repressors. First described in Drosophila melanogaster as important regulators of segmentation, it is clear that PcG genes, and hence protein structure, their repressive function and their targets are conserved throughout evolution. PcG proteins form large macromolecular complexes that combine intrinsic with associated epigenetic activities. PcG repressive complex 2 (PRC2) for instance harbors EZH, a SET domain protein with histone H3K27-directed methyltransferase (HMT) activity. This PcG associated PTM is recognized and engaged by specific chromobox proteins (CBX) in the PRC1 complex. Both PRCs comprise many other factors relevant for regulation of transcription (see table 1). In the fruit fly larvae PRC2 is believed to initiate PcG-based gene silencing, whereas PRC1 takes over as a maintenance complex ${ }^{13}$. Although experimental models, seemingly with very few exceptions, define PcG as transcriptional repressors, how PRCs control repression of promoter activity is mechanistically far from completely understood. The now somewhat antiquated notion that PcG silencing is based on chromatin compaction (i.e. higher order structures) and consequently reduced accessibility for transcription factors is based on steric principle, finds limited experimental support ${ }^{14}$. Recent studies identifying PcG association with the general transcription factory machinery provide new insights into PcG silencing mechanisms. In the fruit fly, PcG act by assembling on Polycomb Responsive Elements (PREs), DNA sequences that can regulate silencing of a nearby promoter in a PcG-dependent fashion ${ }^{15}$. PcG silencing only slightly reduces accessibility of restriction enzymes to the promoter and binding of TATA-binding-protein (TBP) and Rpol2 to the HSP26 promoter was not lost, arguing against chromatin compaction as a major feature in PcG-mediated repression. Drosophila PRC1 proteins interact with several TBPassociated factors (TAFs), that complex with the general transcription factor TFIID 16,17. Furthermore, the Xenopus eed homologue, a PRC2 member, interacts with $\mathrm{TAF}_{\|} 32$, a key component of the TFIID complex in Xenopus ${ }^{18}$. Relevantly, Rpol2 still associates with PcGsilenced promoters but is inhibited in its ability to initiate transcription ${ }^{16}$. Trithorax group proteins are epigenetic activators known to contain histone methyl transferase activity for $\mathrm{H} 3 \mathrm{~K} 4$, which is correlated with active transcription. Interestingly, trithorax proteins activate transcription by counteracting PCG function and by promoting the early phase of transcription elongation ${ }^{19}$. Although this points to PCG silencing taking place at primary chromatin fibers, the seemingly paradoxical co-occurrence of PCG proteins and RPOLII at repressed promoters, and conversely, the finding that numerous $\mathrm{H} 3 \mathrm{~K} 27$ me3-associated promoter are transcriptionally active ${ }^{20,21}$ indicate there is more to PRC-mediated repression than meets the eye. An overview of PcG-associated proteins involved in regulation of transcription is presented in table 1. 


\section{Epigenetics in replication}

DNA replication serves to duplicate DNA in cells in preparation of mitosis. DNA replication is controlled by mechanisms highly conserved throughout evolution. The first step in replication initiation is licensing of replication by recruitment of multiprotein complexes to discrete foci within the nucleus, called replication origins. Replication origins acquire replication competence during $\mathrm{G} 1$ phase in eukaryotes. Binding of the origin recognition complex (ORC) functionally defines DNA replication origins by formation of a pre-replication complex (pre-RC) through complexation with Cdc6 and Cdt1, a replication licensing factor that recruits the MCM2-7 helicase complex. Subsequently, additional proteins including Cdc45 and DNA polymerases join the pre-RC to form the replication fork. The assembly of these multiprotein complexes at replication origins signals the beginning of S-phase. The helicase activity of the complex unzips the DNA, and next the ssDNA is bound by the heterotrimeric protein RPA preventing the DNA from winding back on itself. The ringstructured replication factor PCNA is loaded onto DNA and associates with topoisomerases and replicative DNA polymerases. By sliding along the DNA ahead of the replication fork PCNA enhances the processivity of enzymes in the replication fork ${ }^{22,23}$.

Termination of replication involves Geminin (Gmnn). Gmnn binds free Cdt1, and prevents access of the MCM complex to Cdt1, thereby preventing the initiation of novel pre-RC's. As such Gmnn prevents inappropriate reinitiation of replication, and thus ensures that DNA is replicated only once. Gmnn expression commences at the onset of S-phase and its expression is maintained until the end of mitosis; subsequent inactivation of Gmnn then allows the cell to prepare for a new round of replication ${ }^{23,24}$.

Several lines of evidence implicate chromatin structure in spatial and temporal regulation of DNA replication. As for transcription, chromatin needs to be made accessible for DNA replication. (Hetero)chromatin needs to be decompacted and DNA needs to unwind for replication to occur. Although very little is known about chromatin states around ORCs and pre-RC complexes, this is likely to involve some form of epigenetic regulation. Cell cycle dependent chromatin remodelling is most dramatically visible at the G2/M transition, when chromosomes condense. Although relatively little is known about epigenetic changes that accompany this condensation, some well-known histone-PTMs precede chromosome condensation, e.g. H3S10, H3S28 and H2AX phosphorylation. Many chromatin associated proteins are also phosphorylated in $\mathrm{mid} / \mathrm{late} S$ and released from chromatin. Combined phosphorylation may be required for ensuring chromosome condensation and/or new transcriptional states are epigenetically defined at this time point in the cell cycle when chromatin is assembled. Not only DNA is replicated faithfully, epigenetic information is also transferred from one cell to the other. Chromatin-proteins, like histones, are believed to play a crucial role in this cross-generational transcriptional memory. DNA replication commences at the onset of S-phase and is subject to dynamic programming. Classic nucleotide analogue pulse-labelling experiments have established that DNA replication is differentially regulated throughout the nucleus, defining characteristic S-phase replication patterns in space and 
Chapter 2

time. In 1963 Hay and Revel showed that euchromatin is replicated early, whereas heterochromatin is replicated late in S-phase ${ }^{25}$. In line with this, DNA replication occurs early in Sphase within genomic regions which are transcriptional active, whereas late replicating DNA is found in transcriptionally inactive regions ${ }^{26}$. Goldman showed that housekeeping genes, active in all cells are replicated during the first half of S-phase ${ }^{27}$. Also, tissue-specific genes are replicated early in tissues in which their transcriptional activity is high, whereas non-tissue specific genes are replicated late ${ }^{27}$. As this differential replication is maintained over subsequent rounds of replication and cell division ${ }^{28}$, it seemed reasonable to assume that structural organization of chromatin defines both transcriptional status and replication timing. Hence replication timing profiling may provide information about the developmental status and lineage potential of a cell. Indeed, recent comparisons of replication timing of lineage specific and non-specific genes between pluripotent embryonal stem cells (ESCs) and uni-potent T lymphocytes or neural cells revealed that replication timing is closely linked to lineage restriction and developmental status ${ }^{20,29}$.

The most direct evidence for coordinate tuning of transcription and replication comes from 3Dfluorescence imaging studies in mouse and human diploid fibroblasts. Euchromatic DNA replication and transcription are constrained to defined nuclear areas, indicating that an underlying genomic architecture, likely involving epigenomic marking, segregates the two processes in time and place ${ }^{30}$. Combined the data shows that replication timing is not a static process but that it dynamically shifts across the genome in the contexts of commitment and terminal differentiation ${ }^{20,29}$. Thus, replication timing is an intrinsic cellular feature and is closely linked to transcription. A perhaps logical consequence of this is the notion that transcription and replication need to be tuned to each other during replication. This raises a number of questions: 1) is replication timing a consequence of chromatin structure regulation in the context of transcription? or 2) vice versa does replication timing provide a cellular mechanism to transmit epigenetic information to the next generation, with transcriptional regulation as a consequence? 3) What epigenetic mechanisms are responsible for definition and proper coordinated regulation of both transcription and replication throughout S-phase?

The first cause-or-consequence issues have been studied extensively in the mammalian $\beta$-globin locus. The $\beta$-globin locus is replicated form a single bidirectional origin, firing early in S-phase in erythroid cells, and late in all other cell types ${ }^{31}$. Transcriptional activity of the $\beta$-globin locus is controlled by a locus control region (LCR). Although loss of the LCR at the $\beta$-globin locus significantly reduces transcription of the $\beta$-globin genes chromatin structure, measured by Dnasel sensitivity, and replication timing are unaffected ${ }^{32}$. This suggests that at least for the $\beta$-globin locus, replication timing is not defined by transcriptional status. However, whether specific histone modifications at the LCR are involved in transcriptional regulation of the $\beta$-globin locus, and whether those participate in replication timing is currently unknown.

To begin to probe causal and consequential relationships between transcription and replication timing, it was proposed that chromatin structure is (re)established by virtue of differential 
expression of chromatin modifiers (epigenators; ${ }^{2}$ ) at specific time points during S-phase ${ }^{26,33}$. Proteins involved in assembly of euchromatin would be expressed early in S-phase where proteins involved in heterochromatin formation would be expressed late in S-phase. In support of this, the de novo DNA methyltransferase DNMT1, a transcriptional repressor implicated in heterochromatin formation, targets DMAP1, a transcriptional co-repressor, to replication foci during the entire Sphase. Also HDAC2 associates with DNMT1 and DMAP1, although this interaction was restricted to late S-phase ${ }^{34}$. Thus, factors involved in the formation of transcriptionally repressed epigenetic state are recruited to chromatin during the period in S-phase when heterochromatin is duplicated. It is conceivable that e.g. micro-environmental cues (i.e. morphogens, growth/differentiation factors, cell-cell contacts) may provide the signalling required for (re)activation of epigenators and tissue specific transcription factors to lineage relevant genes and networks ${ }^{2}$. At the bases of coordinated regulation of DNA-templated processes, the assumption that euchromatin is readily available and accessible for replication, and is consequently replicated early, whereas the heterochromatin fraction is less accessible and needs to be remodeled before replication can take place may be a workable starting point. X-chromosome inactivation provides a model to study epigenetic mechanisms in replication timing and transcriptional inactivation. Differentiating female embryonic stem cells inactivate one of two X chromosomes through mechanisms involving a non-coding RNA, called Xist. Xist covers the entire inactive $\mathrm{X}(\mathrm{Xi})$ chromosome ${ }^{35}$. The $\mathrm{Xi}$ is hypoacetylated on histone 3 and 4, shows increased H3K9 and DNA methylation, and relevantly, $\mathrm{Xi}$ is replicated late in $S$ phase ${ }^{36}$. Interestingly, mutations in the DNA methyltransferase $d n m t 3 b$ in female lymphoblasts are associated with DNA hypomethylation and shift Xi-linked gene replication to earlier time points in S-phase ${ }^{37}$. In keeping with this, inhibition of DNA methylation results in both reactivation of gene expression of $\mathrm{Xi}$ genes and advanced replication timing ${ }^{38}$. Although epigenetic mechanisms are clearly involved, not one chromatin modification perfectly correlates with replication timing, hence there must be some degree of flexibility to replication timing and/or additional mechanisms must be responsible for regulation of replication timing in relation to transcription.

\section{Polycomb in replication}

During development expression patterns have to be maintained through several rounds of cell divisions. Histone modifiers and modifications provide a mechanism by which cellular memory of gene expression can be inherited to next cell generations. Polycomb proteins and their counterparts Trithorax proteins are known to be involved in transmitting epigenetic information to next generations during replication ${ }^{39}$. During every cell cycle, when DNA is replicated in S-phase, chromatin-associated proteins and histone modifications are diluted, and need to be re-established on the newly formed daughter strands. Current models imply that modified parental histones are randomly distributed across the two replicating daughter strands ${ }^{40}$. Interestingly, besides for PRC1, H3K27me3 acts as a recognition mark for the PRC2 complex; this suggests a possible 


\section{Chapter 2}

mechanism for transmission of H3K27me3 marks by PRC2 to newly assembled nucleosomes ${ }^{41}$. Recent studies also suggest a more direct role for PcG proteins in DNA replication. Drosophila mutants for PRC1 members Ph, Pc and Psc, and the Enhancer of Trithorax and Polycomb (ETP) gene Asx show severe chromatin segregation defects in syncytial embryos ${ }^{42}$. Although poorly understood, these and other earlier observations provided an association between PRC-function and DNA replication ${ }^{42}$. Importantly, Drosophila Grp/Chk1 and Chk2 (DmChk2) mutants also accumulate segregation defects, as a result of failure to activate checkpoint (metaphase to anaphase delay) in response to DNA damage or incompletely replicated DNA ${ }^{43}$. These raises the question whether the accumulation of segregation defects in PcG mutant Drosophila is the direct consequence of problems in DNA replication and whether a DNA damage response is involved (please see discussion below).

Direct and genetic interactions exist between PcG proteins and proteins involved in regulating DNA replication. Topoisomerase II (Topo2), well-known for its role in replication, and Barren (BARR), a potential activator of Topo2 ${ }^{44}$, both associate with genomic PcG target sequences in Drosophila ${ }^{45}$. In line with the finding that Topo2 and Barren bind PREs, they directly bind to the PcG protein Polyhomeotic (Ph). In addition, Cramped (CRM), a Drosophila PcG protein, localizes to the nucleus during S-phase and CRM expression disappears at mitosis. The cell cycle-dependent localization resembles that of PCNA and suggested a functional relationship. Indeed, CRM and PCNA colocalize on salivary gland polytene chromosomes and a double mutant for crm and pcna (Drosophila mus209 allelle), displays an exaggerated phenotype in anteroposterior and distalproximal transformations. Besides shared function, more importantly, this illustrates that defective replication is a determining factor in abnormal segmentation ${ }^{46}$. In further support of functional kinship, a transient association of Gmnn with the mammalian PRC1 members Scmh1 and Phc1/Mphc/Rae28 was recently discovered ${ }^{47}$. And finally, the mammalian PcG protein Bmi1 was identified as a direct interactor of the replication licensing factor CDC6: the transition of late to early replication of the INK4A/ARF locus in senescing embryonic fibroblasts underscores the functional association between PCG and replication timing ${ }^{48}$. Overall, whilst experimental proof in this area will undoubtedly expand, the collective data suggests that PcG function is directly implicated in regulation of replication. An overview of $\mathrm{PcG}$-associated proteins involved in regulation of replication and repair is presented in table 1.

\section{Coordinated regulation replication and transcription by PcG}

Although PcG proteins are classically associated with transcriptional repression, mounting evidence supports a broader role for PCG in other DNA templated processes besides transcriptional regulation. In Drosophila, regulatory elements of lab-4 and mcp are enriched for both Polycomb (PC) and Trithorax (TRX) early in embryogenesis, while seemingly in conflict with the classic view that PRC alter chromatin for transcriptional repression, both genes are transcriptionally active ${ }^{49}$. Analogously, bivalently marked promoters in murine embryonic stem 
cells, carrying both repressing $(\mathrm{H} 3 \mathrm{~K} 27 \mathrm{me} 3)$ and activating $(\mathrm{H} 3 \mathrm{~K} 4 \mathrm{me} 3)$ marks, were found to be transcriptionally active, albeit at low levels ${ }^{21}$. We recently found evidence for significant transcriptional induction of putative PcG-target genes in vitro and in vivo, despite persistence of H3K27me3 marks; in vitro phospho-dependent release of PRC1-complex members correlated with activation of expression $\left({ }^{50}+\right.$ in preparation). These findings begin to show that the role of $\mathrm{H} 3$ trimethylation, and possibly PRC1 function, exceeds that of transcriptional silencing only.

Cells undergo replication stress under conditions that affect replication fork progression during Sphase. As stalled replication can be mutagenic and induce cell death, several pathways have evolved to repair damaged DNA and to get collapsed replication forks back on track ${ }^{51,52}$. Activation of sophisticated intraS-phase checkpoints allows cells to resolve replication stress and DNA damage, and to complete $S$ phase to allow cells to proceed to mitosis ${ }^{53}$. The majority of DNA damage in replicating cells, even under normal conditions, probably comes from highly frequent collision between replication forks and transcription machineries, as both processes move along the same template ${ }^{51,54}$. Hence, these processes need to be tightly regulated to prevent DNA damage. Under conditions of rapid proliferation, e.g. in response to enhanced mitogenic signalling by, among others, oncogenic E2Fs, cMyc, Ras, Braf, and RUNX, the increased replication rate puts a tremendous strain on chromatin. Upon initial oncogene-induced hyperproliferation, sustained expression of oncogenes in fibroblasts activates a senescence response termed oncogene-induced senescence (OIS) ${ }^{55,56}$. In support of the notion that under hyperproliferative conditions, cells are unable to cope with the stress that the increased demand for DNA replication puts on chromatin, cells expressing oncogenic RasV12, for example, amass replication-dependent double strand DNA breaks (DSB) and accumulate stalled replication forks; senescent cells harbour senescence-associated DNA-damage foci (SDF), containing ATM, NBS1, ATR, ATRIP and RPA ${ }^{56}$. Activation of a DNA damage response (DDR) is instrumental in the induction of OIS, as knockdown of various DDR proteins (ATM, ATR, Chk1, Chk2 and p53) rescues proliferation ${ }^{56}$. Intriguingly, co-expression of Bmi1 in Ras $\mathrm{V} 12$ expressing cells bypasses senescence to a large extent ${ }^{57}$. Although mechanistically not worked out in detail, analogy with oncogenic cooperation between c-Myc and Bmi1 points to a possible involvement of INK4A/ARF suppression by Bmi ${ }^{58}$. Recent data from our group support a role for PRC1 in replicative control and begins to shed some light on underlying mechanisms. In analogy to expression of oncogenes in fibroblasts, activation of differentiation in vitro often sets off a transient hyperproliferative phase commonly referred to as transit amplification TA: transit amplifying cells (TAC) provide the necessary expansion of progenitor populations to provide for adequate tissue repair/replacement from limited numbers of mobilized stem cells (SC). Although hard to pinpoint in vivo, TA has been suggested to underlie the rapid regeneration of tissues, among which intestinal villi from SCs located in the crypts of Lieberkühn ${ }^{59}$. One of the few places in the body where TAC are readily identifiable is the proliferative zone in the developing skeleton: during endochondral ossification (the formation of bony skeletal elements from cartilage scaffolds) histologically distinctive tracts of rapidly 


\section{Chapter 2}

proliferating cells are readily visible ${ }^{60}$. TA can be mimicked in vitro, in chondrogenic models: the mesenchymal stem cell ATDC5 will undergo a proliferative burst upon induction of differentiation ${ }^{61}$. Despite the characteristic effect of many PRC1 null-mutations on antero-posterior skeletal morphogenesis in mice ${ }^{62}$, a direct role for PRC1 in chondrogenesis was never established. Unexpectedly, PRC1-deficient ATDC5 cells fail to undergo TA, and activate an intraS-phase block in response to massive accumulation of DNA damage. PRC1-KD cells are very sensitive to replication poisons; accumulate DSB at sites of de novo DNA synthesis and ultimately senesce, exposing a causal relationship between defective PRC1-fuction and replication induced DNA damage (this thesis).

An obvious question is: how does PRC1 prevent replication stress? A number of observations points in the direction of coordinated regulation of transcription and replication during TA. Whereas G1 nuclei typically stain brightly for Ser5-phosphorylated Rpol2 (pRpol2), cells progressing through S-phase normally undergo a transition from genome-wide transcriptional activity to complete shutdown of transcription initiation at mid/late S-phase. Co-staining for $\mathrm{BrdU}$ and $\mathrm{pRpol} 2$ revealed enhanced BrdU incorporation at sites where transcription initiation was low, or put differently, low transcriptional activity at site of DNA replication $\left({ }^{30}\right.$, this thesis). Remarkably, this functional genome partitioning is completely lost in S-phase arrested Bmi1-deficient cells. In addition, cells carrying DSB display a generally deregulated transcription, which is also reflected in comparative expression array analysis in the context of differentiation: transcription levels of many genes were consistently upset throughout differentiation; in addition, genes irrelevant to chondrogenesis were induced, whereas other genes that should have been induced were not. Although deregulation of specific pathways awaits further analysis, it is clear that redefinition of transcription and replication activity are closely coupled during chondrogenesis and that somehow PRC1 is required to facilitate differentiation under hyperproliferative conditions.

Recently, PRC1 was identified as an E3 ligase for Gmnn: Rnf2/Bmi1-mediated ubiquitylation of Gmnn targets it for proteasomal degradation. In vivo, loss of the PRC1 protein Phc1, through which Gmnn is associated to PRC1 and a direct interactor of Bmi1 and Rnf2, results in stabilization of $\mathrm{Gmnn}$ and blocks haematopoietic stem cell proliferation ${ }^{63}$. Stabilization of $\mathrm{Gmnn}$ indeed prevents cell from completing their cell cycle ${ }^{64}$. To explain the leukopenia in Phc1-null mice, PRC1mediated control of Gmnn expression was suggested to affect the proliferative potential and/or differentiation capacity of HSC ${ }^{65}$. The earlier described physical interaction between PcG complexes with elements of transcription factories as well as replication machineries, in addition to the physical and biochemical interactions between PRC1 and Gmnn, suggest a mechanism for PRC1-mediated orchestration of transcription in respect to replication and/or vice versa. We here propose a model in which PCR1/Gmnn acts as a molecular switch between transcriptional and replicative choices in mid-S phase (figure 1): H3K27me3-mediated recruitment of the core complex CBX/Rnf2/Bmi1 installs repressive H2AK119ub1 marks, which interfere with transcription initiation, possibly through recruitment of additional PRC (associated) proteins ${ }^{1}$. Local presence of 
Rnf2/Bmi1 E3 ligase activity would target Gmnn for degradation, relieving a potential replication block. Conversely, loss of Bmi1/Rnf2 stabilizes GMNN, hence blocking replication, yet permitting transcription. Chromatin dissociation of Bmi1/Rnf2 is likely controlled by post-translational modification (PTM). In line with this idea, we recently observed a positive correlation between PRC-phosphorylation, PRC1/chromatin dissociation and Gmnn stabilization under various conditions of cell stress (JWV, YT unpublished observations). Stress signaling induces chromatin dissociation of PRC1 members (Bmi1, Ring and CBX proteins; ${ }^{50}$ ). In contrast, Phc1/Rae28 ${ }^{50}$ undergoes a nuclear redistribution under these conditions and may provide a docking point for Gmnn stabilization.

As indicated before, Gmnn levels gradually increase toward late S-phase and chromatin-bound Gmnn blocks rereplication of DNA at the in G2/M. Increased Gmnn-chromatin association correlates with PRC1 dissociation from chromatin during mid S-phase ${ }^{66}$. Relevantly, Bmi1deficient chondrogenic progenitors arrest in midS-phase and display significantly increased Gmnn chromatin association (this thesis). The inverse correlation between Gmnn and Bmi1/Rnf2 chromatin association during mid-S is in perfect agreement with the ubiquitin-mediated induction of Gmnn proteolysis by Bmi1/Rnf2 ${ }^{65}$. Relevantly, the most dramatic replication-retiming happens at mid-S-phase in the context of differentiation, when replication origins are spatially redefined and replication timing and transcription are re-tuned ${ }^{26}$. The dynamic relocation of PRC1 and Gmnn during this phase suggests a functional connection with replication timing in the context lineage commitment and differentiation from a pluri- or multipotent stem cell.

Of note, the primary task of H3K27me3 (and possibly H3K4me3) in the proposed model would not be to mediate transcriptional repression (or activation, respectively) per se. Rather global (and local) histone trimethyl (and possibly additional) marking may provide the required epigenomic context for tuning transcription and replication. Our observation that cell stress consistently coinduces global $\mathrm{H} 3$ trimethylation at $\mathrm{K} 4, \mathrm{~K} 27$ and $\mathrm{K} 9$, supports the idea that these marks regulate more than transcription. Hence, PcG and trxG-associated epigenetic marks may functionally partition the genome and help coordinate DNA-templated processes, for example in the context of development.

\section{Concluding remarks}

Early observations in Drosophila melanogaster, although incompletely understood, provided an early indication of a link between PRC-LOF phenotypes and DNA replication ${ }^{42}$. We here provide a tentative explanation for the replication defects in PRC-mutants: PRC1 orchestrates chromatinbased processes, such as increased transcriptional reprofiling and enhanced DNA replication during transit amplification in the context of differentiation. The consequence of this observation in relation to the etiology of PRC1 phenotypes in vivo is as of yet unclear. PRC1 proteins classically maintain HOX gene expression boundaries set by segmentation and pair-rule genes, and skeletal phenotypes correlate with defective maintenance of HOX gene expression boundaries ${ }^{67}$. In line 
Chapter 2

with published data on regulation of proliferation by HOX-genes, in addition to long-standing speculations about contribution of a proliferative component to axial and appendicular phenotypes in HOX mutants, it is possible that proliferation and HOX gene expression both contribute to abnormal replication ${ }^{68}$. However, since no two single PRC1 mutants affect the same HOX genes; although by and large, most PCR1-null mutants display relatively similar posterior transformations, this automatically begs the question whether HOX deregulation may be consequential rather than causal in this context. Multiple additional interesting questions arise: What is the exact molecular nature of functional genome partitioning? Does replication timing determine transcriptional activity, or vice versa? Is coordination of transcription and replication subject to clustered regulation, i.e. groups of genes or networks in the context of lineage commitment, and how exactly are PRCs implicated in these matters? What post-translational modifications and protein interactions help direct PRC activity toward transcriptional repression or replication? Can the findings reported herein be readily projected onto embryonal stem cells, i.e. does failure to properly coordinate simultaneous DNA templated processes contribute to self-renewal defects? And, in the context of OIS, is PRC1 inactivation involved in OIS or, conversely could Polycomb overexpression in cancer be required for protection during hyperreplication? Chromatin has been called the most relevant physiological substrate in a cells' life. Coordinated regulation of processes like transcription, replication and DNA repair is important to prevent DNA damage and help maintain genome integrity, cell identity and provide regenerative capacity. As all these processes use one and the same template, DNA, it should come to no surprise that many regulatory components are shared, including Polycomb group proteins and geminin. An important future challenge will be to determine the exact molecular mechanisms and contributions of Polycomb Group proteins at the cross road of chromatin templated processes, like transcription and replication. 


\begin{tabular}{|c|c|c|c|}
\hline Drosophila & Mammals & Associated proteins & Process \\
\hline$E(z)$ & Ezh1/Ezh2 & Xnp ${ }^{69}$ & DNA repair \\
\hline Esc & Eeed & $\mathrm{TAF}_{\| \mid} \mathrm{D}\left(\right.$ Xenopus) ${ }^{18}$ & Transcription \\
\hline Su(z)12 & Suz12 & & \\
\hline $\mathrm{Pc}$ & $\mathrm{Cbx} 4, \mathrm{Cbx} 6, \mathrm{Cbx} 7, \mathrm{Cbx} 8$ & $\mathrm{TBP}^{11}$ & Transcription \\
\hline $\mathrm{Pcl}$ & Phf1 & $\begin{array}{l}\text { Rad50, Smc1, Dhx9 and } \\
\text { p53 }\end{array}$ & DNA repair \\
\hline $\mathrm{Ph}$ & Phc1/Phc2/Phc3 & $\begin{array}{l}\text { Tbp }{ }^{1 /}, \mathrm{Gmnn}^{41} \\
\text { Topo2, }^{41}{ }^{4}{ }^{4}\end{array}$ & Transcription/Replication \\
\hline Sce/dRing & Ring1A/Rnf2 & $\mathrm{Tbp}^{11}$ & Transcription \\
\hline Psc & Bmi1/ Mel18 & $\operatorname{Tbp}^{17}, \mathrm{Gmnn}^{47}, \mathrm{Cdc} 6^{48}$ & $\begin{array}{l}\text { Transcription/ } \\
\text { Replication }\end{array}$ \\
\hline Pho & YY1 & $\mathrm{Tbp}^{16}$ & Transcription \\
\hline Smc & Smch1 & $\mathrm{Gmnn}^{4 \prime}$ & Replication \\
\hline Crm & & Pcna ${ }^{46}$ & Replication \\
\hline & Rybp & $\mathrm{Mdm}^{11}$ & DNA repair \\
\hline
\end{tabular}

Table 1 Overview PcG binding proteins

Figure 1

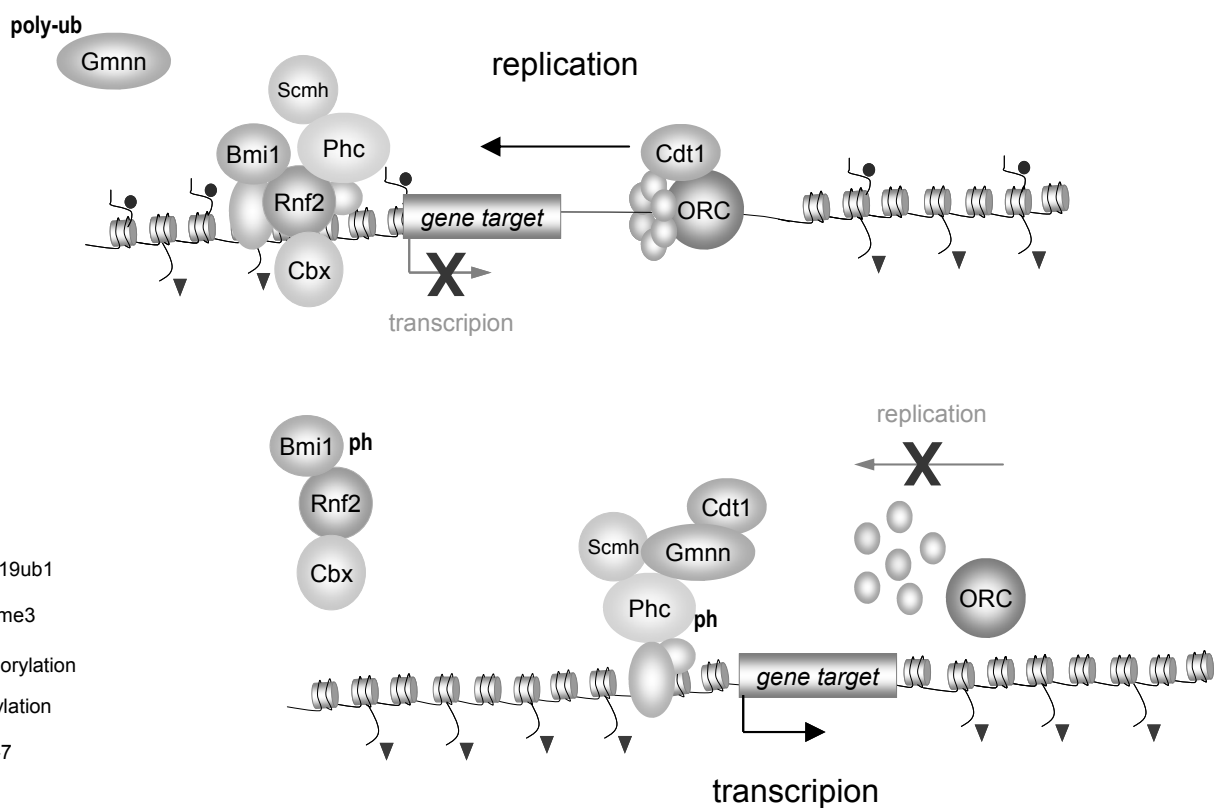

Figure 1. Proposed model for segregation of transcription and replication by PcG proteins.

H3K27me3-mediated recruitment of the core complex CBX/Rnf2/Bmi1 installs repressive H2AK119ub1 marks, which interfere with transcription initiation, possibly through recruitment of additional PRC (associated) proteins 1. Local presence of Rnf2/Bmi1 E3 ligase activity would target Gmnn for degradation, relieving a potential replication block. Conversely, loss of Bmi1/Rnf2 stabilizes GMNN, hence blocking replication, yet permitting transcription. 


\section{Acknowledgements}

The authors apologize to their colleagues whose relevant work was not cited. The authors thank M. Vidal (Madrid, Spain) and R.Shiri-Sverdlov for critically reading the manuscript. The authors received financial support from the European Molecular Biology Organization (Germany) ASTF52009; the Dutch Science Organization (ZonMW-NWO) VIDI grant 016.046.362, the National Rheuma Foundation (Reumafonds) grant LLP14 and transnational University of Limburg (tUL) grant to JWV.

\section{References}

1. Garcia, E. et al., Embo J 18 (12), 3404 (1999); Arrigoni, R. et al., FEBS Lett 580 (26), 6233 (2006).

2. Berger, S. L., Kouzarides, T., Shiekhattar, R., and Shilatifard, A., Genes Dev 23 (7), 781 (2009).

3. Groth, A., Rocha, W., Verreault, A., and Almouzni, G., Cell 128 (4), 721 (2007).

4. $\quad$ Kouzarides, T., Cell 128 (4), 693 (2007).

5. Thomas, M. C. and Chiang, C. M., Crit Rev Biochem Mol Biol 41 (3), 105 (2006).

6. Sandelin, A. et al., Nat Rev Genet 8 (6), 424 (2007).

7. Li, B., Carey, M., and Workman, J. L., Cell 128 (4), 707 (2007).

8. $\quad$ Buratowski, S., Nat Struct Biol 10 (9), 679 (2003).

9. $\quad$ Cairns, B. R., Nat Struct Mol Biol 14 (11), 989 (2007).

10. Dinant, C., Houtsmuller, A. B., and Vermeulen, W., Epigenetics Chromatin 1 (1), 9 (2008).

11. Wysocka, J. et al., Nature 442 (7098), 86 (2006).

12. Goodarzi, A. A. et al., Mol Cell 31 (2), 167 (2008).

13. Schwartz, Y. B. and Pirrotta, V., Nat Rev Genet 8 (1), 9 (2007).

14. Dellino, G. I. et al., Mol Cell 13 (6), 887 (2004).

15. .Francis, N. J. and Kingston, R. E., Nat Rev Mol Cell Biol 2 (6), 409 (2001); Mahmoudi, T., Zuijderduijn, L. M., Mohd-Sarip, A., and Verrijzer, C. P., Nucleic Acids Res 31 (14), 4147 (2003); Mohd-Sarip, A., Venturini, F., Chalkley, G. E., and Verrijzer, C. P., Mol Cell Biol 22 (21), 7473 (2002).

16. Breiling, A., Turner, B. M., Bianchi, M. E., and Orlando, V., Nature 412 (6847), 651 (2001).

17. Saurin, A. J. et al., Nature 412 (6847), 655 (2001).

18. Showell, C. and Cunliffe, V. T., Gene 291 (1-2), 95 (2002).

19. Srinivasan, S. et al., Development 132 (7), 1623 (2005); Srinivasan, S., Dorighi, K. M., and Tamkun, J. W., PLoS Genet 4 (10), e1000217 (2008).

20. Azuara, V. et al., Nat Cell Biol 8 (5), 532 (2006).

21. Bernstein, B. E. et al., Cell 125 (2), 315 (2006).

22. Bambara, R. A., Murante, R. S., and Henricksen, L. A., J Biol Chem 272 (8), 4647 (1997); Burgers, P. M., J Biol Chem 284 (7), 4041 (2009); Kunkel, T. A. and Bebenek, K., Annu Rev Biochem 69, 497 (2000); Moldovan, G. L., Pfander, B., and Jentsch, S., Cell 129 (4), 665 (2007); Nitiss, J. L., Nat Rev Cancer 9 (5), 327 (2009); Stillman, B., Cell 78 (5), 725 (1994).

23. Nishitani, H. and Lygerou, Z., Genes Cells 7 (6), 523 (2002).

24. Lee, C. et al., Nature 430 (7002), 913 (2004).

25. Hay, E. D. and Revel, J. P., J Cell Biol 16, 29 (1963).

26. Gilbert, D. M., Curr Opin Cell Biol 14 (3), 377 (2002).

27.

28.

29.

30.

31.

32.

Ma, H. et al., J Cell Biol 143 (6), 1415 (1998).

Perry, P. et al., Cell Cycle 3 (12), 1645 (2004).

Wei, X. et al., Science 281 (5382), 1502 (1998).

Aladjem, M. I. et al., Science 270 (5237), 815 (1995); Simon, I. et al., Embo J 20 (21), 6150 (2001). Cimbora, D. M. et al., Mol Cell Biol 20 (15), 5581 (2000).

Hiratani, I., Takebayashi, S., Lu, J., and Gilbert, D. M., Curr Opin Genet Dev 19 (2), 142 (2009).

Rountree, M. R., Bachman, K. E., and Baylin, S. B., Nat Genet 25 (3), 269 (2000).

Ng, J. H., Heng, J. C., Loh, Y. H., and Ng, H. H., Mutat Res 647 (1-2), 52 (2008).

Keohane, A. M. et al., Dev Biol 180 (2), 618 (1996); Wutz, A. and Jaenisch, R., Mol Cell 5 (4), 695 (2000).

37. Hansen, R. S. et al., Hum Mol Genet 9 (18), 2575 (2000).

38. Subramanian, P. S. and Chinault, A. C., Somat Cell Mol Genet 23 (2), 97 (1997).

39. Bantignies, F. and Cavalli, G., Curr Opin Cell Biol 18 (3), 275 (2006); Mahmoudi, T. and Verrijzer, C. P., Oncogene 20 (24), 3055 (2001). 
40. Probst, A. V., Dunleavy, E., and Almouzni, G., Nat Rev Mol Cell Biol 10 (3), 192 (2009); Nakatani, Y., Tagami, H., and Shestakova, E., Ernst Schering Res Found Workshop (57), 89 (2006).

41. Hansen, K. H. et al., Nat Cell Biol 10 (11), 1291 (2008).

42. O'Dor, E., Beck, S. A., and Brock, H. W., Dev Biol 290 (2), 312 (2006).

43. Royou, A., Macias, H., and Sullivan, W., Curr Biol 15 (4), 334 (2005); Xu, J. and Du, W., FEBS Lett 545 (2-3), 209 (2003).

44. Bhat, M. A., Philp, A. V., Glover, D. M., and Bellen, H. J., Cell 87 (6), 1103 (1996).

45. Lavoie, B. D. et al., Mol Biol Cell 11 (4), 1293 (2000).

46. Yamamoto, Y. et al., Development 124 (17), 3385 (1997).

47. Luo, L. et al., Nature 427 (6976), 749 (2004).

48. Agherbi, H. et al., PLoS One 4 (5), e5622 (2009).

49. Orlando, V. et al., Embo J 17 (17), 5141 (1998).

50. Voncken, J. W. et al., J Biol Chem 280 (7), 5178 (2005).

51. Brewer, B. J., Cell 53 (5), 679 (1988).

52. Cox, M. M. et al., Nature 404 (6773), 37 (2000).

53. Giono, L. E. and Manfredi, J. J., J Cell Physiol 209 (1), 13 (2006); Chen, Y. and Poon, R. Y., Front Biosci 13, 5016 (2008).

54. Rudolph, C. J., Dhillon, P., Moore, T., and Lloyd, R. G., DNA Repair (Amst) 6 (7), 981 (2007); Mirkin, E. V. and Mirkin, S. M., Microbiol Mol Biol Rev 71 (1), 13 (2007).

55. Bartkova, J. et al., Nature 444 (7119), 633 (2006).

56. Di Micco, R. et al., Nature 444 (7119), 638 (2006).

57. Datta, S. et al., Cancer Res 67 (21), 10286 (2007).

58. Jacobs, J. J. et al., Genes Dev 13 (20), 2678 (1999).

59. Pinto, D. and Clevers, H., Exp Cell Res 306 (2), 357 (2005).

60. Mackie, E. J. et al., Int J Biochem Cell Biol 40 (1), 46 (2008).

61. Atsumi, T., Miwa, Y., Kimata, K., and Ikawa, Y., Cell Differ Dev 30 (2), 109 (1990).

62. Akasaka, T. et al., Development 122 (5), 1513 (1996); del Mar Lorente, M. et al., Development 127 (23), 5093 (2000); Alkema, M. J. et al., Nature 374 (6524), 724 (1995).

63. Ohtsubo, M. et al., Proc Natl Acad Sci U S A (2008).

64. Yoshida, K., Front Biosci 12, 2984 (2007).

65. Ohtsubo, M. et al., Proc Natl Acad Sci U S A 105 (30), 10396 (2008).

66. Voncken, J. W. et al., J Cell Sci 112 ( Pt 24), 4627 (1999).

67. Beuchle, D., Struhl, G., and Muller, J., Development 128 (6), 993 (2001); del Mar, L. M. et al., Development 127 (23), 5093 (2000); Hanson, R. D. et al., Proc.Natl.Acad.Sci.U.S.A 96 (25), 14372 (1999); Isono, K. et al., Mol Cell Biol 25 (15), 6694 (2005); van der Lugt, N. M., Alkema, M., Berns, A., and Deschamps, J., Mech.Dev. 58 (1-2), 153 (1996); Zhang, H. et al., Nat Genet 36 (5), 507 (2004).

68. Duboule, D., Curr Opin Genet Dev 5 (4), 525 (1995).

69. Cardoso, C. et al., Hum Mol Genet 7 (4), 679 (1998).

70. Hong, Z. et al., Nucleic Acids Res 36 (9), 2939 (2008).

71. Chen, D. et al., EMBO Rep 10 (2), 166 (2009). 
Chapter 2 


\section{Chapter 3}

\section{A novel in vivo model to study enchondral bone formation;}

HIF-1 $1 \alpha$ activation and

\section{BMP expression}

Pieter J. Emans, Frank Spaapen, Don A.M. Surtel, Keryn M. Reilly, Andy Cremers, Lodewijk W. van Rhijn, Sjoerd K. Bulstra, Jan Willem Voncken, Roel Kuijer. 


\begin{abstract}
Numerous growth and transcription factors have been implicated in endochondral bone formation of the growth plate. Many of these factors are up-regulated during hypoxia and downstream of Hypoxia-Inducible Factor (HIF)-1 $\alpha$ activation. However, the specific function of these factors, in the context of oxygenation and metabolic adaptation during adult periosteal endochondral bone

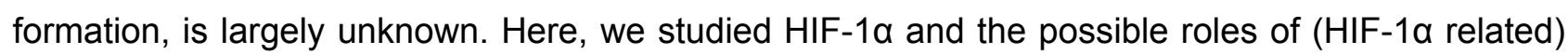
growth and transcription factors in a recently developed in vivo model for adult periosteal endochondral bone formation.

At different phases of periosteal endochondral bone formation, mRNA levels of Transforming Growth Factor (TGF)- $\beta 1$, Bone Morphogenetic Proteins (BMP)-2, -4, and -7, Indian Hedgehog (Ihh), Parathyroid Hormone-related Protein (PTHrP), Sox9, Runx2, HIF-1a, Vascular Endothelial Growth Factor (VEGF), periostin (POSTN), and Glyceraldehyde-3-Phophate Dehydrogenase (GAPDH) were evaluated with RT-real time-PCR. Also protein levels of TGF- $\beta 1$, BMP-2, -4 , and -7 , HIF-1 $\alpha$, and POSTN were examined. During the chondrogenic phase, the expression of Sox9, Ihh, and HIF-1 $\alpha$ was significantly up-regulated. TGF- $\beta 1$ mRNA levels were rather constant, and the mRNA levels of BMPs were significantly lower. Immunohistochemical detection of corresponding gene products, however, revealed the presence of the proteins of TGF- $\beta 1$, BMP-2, -4 , and -7 , HIF$1 \alpha$, and POSTN within the chondrocytes during chondrogenesis. This discrepancy in gene expression between mRNA and protein level for TGF- $\beta 1$ and the different BMPs is indicative of post-transcriptional regulation of protein synthesis. HIF-1 $\alpha$ activation and up-regulation of GAPDH support a hypoxia-induced metabolic shift during periosteal chondrogenesis. Our model recapitulates essential steps in osteochondrogenesis and provides a new experimental system to study and ultimately control tissue regeneration in the adult organism.
\end{abstract}




\section{Introduction}

Endochondral bone formation is a multistage process; early commitment of mesenchymal stem cells (MSC) to a chondrogenic cell fate is accompanied by morphological changes and activation of a cartilage specific gene program. At progressive differentiation stages toward osteogenesis, chondrocytes resume proliferation, mature into hypertrophic chondrocytes, and then calcify their surrounding matrix and undergo programmed cell death ${ }^{1}$. Simultaneously, angiogenesis is stimulated and the calcified cartilage is replaced by bone. Transforming Growth Factor (TGF)- $\beta 1$ and members of its superfamily, among which are the Bone Morphogenetic Proteins (BMPs), play an important role in endochondral bone formation and fracture healing ${ }^{2}$. BMPs are known to interact with the Indian Hedgehog (Ihh)/Parathyroid Hormone related Protein (PTHrP) pathway and exert their function, at least in part, via expressional regulation of Sox9, an essential transcription factor in chondrogenesis ${ }^{1-7}$. In addition, hypoxia, as a micro-environmental determinant, is thought to promote differentiation of mesenchymal cells along a chondrocyte pathway, in part by activating Sox-9 via an HIF-1a-dependent mechanism ${ }^{8}$. Although conditions are hypoxic within the endochondral growth plate ${ }^{9}$, not much information is available on oxygenation and metabolic adaptation during periosteal callus formation. Several observations directly link oxygen tension to osteochondrogenic differentiation. The transcription factor Runx2 (Aml3, Osf2/Cbfa1) is required for differentiation of osteoblast progenitors during the final stages of endochondral bone formation and is also downstream of BMP signalling ${ }^{10,11}$. Runx2 mRNA is subject to IRES-dependent translational control, which is thought to permit continued translation under conditions suboptimal for cap-dependent translation (e.g. under low oxygen concentration) ${ }^{12}$. The cell adhesion protein periostin (POSTN, Osf2-not to be confused with Osf2/Cbfa1) is highly homologous with ßigH3/ $\beta i g H 3$ and is induced by TGF- $\beta 1$ and hypoxia ${ }^{13-16}$. POSTN may play a role in the recruitment and attachment of osteoblast precursors and is specifically found in periosteum and the periodontal ligament ${ }^{14}$. The mRNA expression for this protein is highly up-regulated during fracture healing and also increases with mechanical stress in periodontal ligament tissues ${ }^{17-19}$. Most of these findings stem from in vitro models. A possible connection between oxygenation and expression of these growth and transcription factors during periosteal endochondral bone formation in vivo has so far not been studied. Studies on endochondral bone formation in vivo often use whole embryos or growth plates as paradigms ${ }^{5,6,20}$. In addition, fracture healing models in adult rodents are used in which the bone marrow is penetrated ${ }^{21,22}$. To date, experimental models for endochondral bone formation in adult organisms have, to our knowledge, not been described, whereas such models are likely relevant in respect to clinical situations. We have recently established a novel in vivo model for osteochondrogenesis, in which periosteum is damaged without breaking the bone and accessing the bone marrow ${ }^{23}$. Periosteum contains pluripotent progenitor cells that harbor MSC characteristics ${ }^{24,25}$ and can differentiate to chondrocytes and osteoblasts during normal bone growth and fracture healing ${ }^{26,27}$. We demonstrated the chondro-regenerative capacity of periosteal MSC ${ }^{23}$. This novel model can be used to study the function of periosteum during cartilage and 


\section{Chapter 3}

endochondral bone formation. In addition, the model is expected to provide important mechanistic insight for tissue engineering of cartilage and/or bone and for fracture healing ${ }^{23}$. The aim of this study was to evaluate expression of essential factors, among which is the hypoxia-responsive factor HIF-1 $\alpha$, during the in vivo generation of adult periosteum derived cartilage and bone.

\section{Materials and methods}

\section{Surgical procedures}

Six-month-old female New Zealand White rabbits were used for this study. European and Dutch laws on animal experimentation were strictly followed throughout the study, and the animal experimental protocol was approved by the Maastricht University committee for animal experiments. Surgical procedures were performed under general anesthesia and with the use of sterile techniques. Anesthetic induction was obtained with $35 \mathrm{mg} / \mathrm{kg}$ ketamine hydrochloride and 5 $\mathrm{mg} / \mathrm{kg}$ xylazine hydrochloride administered by intramuscular injection. Anesthesia was maintained by means of a mixture of $2 \%$ halothane and oxygen/nitrous oxide delivered by an automatic ventilator using an especially designed mask. All rabbits received a preoperative intramuscular injection with antibiotics $(10 \mathrm{mg} / \mathrm{kg}$ ceftiofur sodium, Pharmacia and Upjohn, Woerden, The Netherlands). Postoperative analgesia provided by administering $0.05 \mathrm{mg} / \mathrm{kg}$ buphenomorfine intramuscular once a day for 2 days. Bilaterally, the skin and the overlying fascia were opened over the upper medial tibia. A piece of approximately $7 \times 15 \mathrm{~mm}$ of the periosteum was dissected. The harvested tissue of five rabbits was used to assess expression levels of the genes of interest in undifferentiated periosteum ( $\mathrm{t}=0$ day). The overlying fascia was closed with Polysorb 2.0 sutures (Tyco Healthcare, USA). Special care was taken that the tendon of the semitendinosus muscle was sutured on top of the lower, short side of the rectangular periosteum defect. Hereafter the skin was closed with Polysorb 4.0 (Tyco Healthcare, USA). At days 10, 20, and 40 postoperative, the reactive tissue of five rabbits per time point was harvested. For this purpose, the rabbits were anesthetized as described above. The reactive tissue was dissected with a scalpel or osteotome (40 days). Again the overlying fascia and skin were closed with Polysorb (Tyco Healthcare, USA). Specimens were cut in two halves, one part was used for RNA isolation, and the other was fixed in $4 \%$ paraformaldehyde, dehydrated and embedded in paraffin.

\section{RNA isolation}

Immediately after harvest, the tissue was frozen in liquid nitrogen and stored at $-80^{\circ} \mathrm{C}$. The frozen tissue was cooled further in liquid nitrogen and pulverized using a microdismembrator $S$ (Bauer, Goettingen, Germany). The resulting powder was collected in TriZol reagent, and RNAwas extracted according to the protocol of the manufacturer (Invitrogen, Breda, The Netherlands). Quantity and purity of the RNAwere determined 260/280 nm absorbance measurements. The RNA samples were subjected to RNase-free DNase digestion to remove any genomic DNA and to 
agarose gel electrophoresis to assess the intactness of RNA. Complete removal of genomic DNA was verified by PCR.

\section{Rabbit primer design}

Since sequence information on the rabbit genome is limited, conserved human and murine gene sequences were determined to design primer sets for Ihh, Runx2, Sox9, Collagen Type II and Type $X$ and POSTN by using Blast2 software (www.ncbi.nlm.nih.gov). PCR primer sets were designed for homologous gene sequences and tested on rabbit cDNA samples (Supplemental table 1). The identities of the PCR products were confirmed by direct sequencing and comparison with known human and mouse genes and then submitted to GenBank (AY633663, AY598932, AY598933, AY598934, AY598935, AY598936, AY598937).

\section{Semi-quantitative reverse transcription-polymerase chain reaction}

Although multiple real time primer sets were tested, the expression of Collagen Type (COL) X, TGF- $\beta 1$ and Ihh could only be determined with a semiquantitative RT-PCR. To this end, we performed PCR reactions on serially diluted cDNAs using primer sets for Ihh, TGF- $\beta 1$, COLX, either alone or in combination with a primer set for 28S. 28S cDNA amplification was also performed separately. Ethidiumbromide signals were quantified using a GELDoc 1000 system with Multi-Analyst imaging analysis (Bio-Rad, CA, USA). Marker mRNA levels are expressed relative to $28 \mathrm{~S}$ rRNA. Two hundred nanograms of heat denatured RNA was reverse transcribed with $100 \mathrm{U}$ MMLV reverse transcriptase (Promega, WI, USA) in $50 \mathrm{mM}$ Tris- $\mathrm{HCl}(\mathrm{pH} 8.3), 75 \mathrm{mM} \mathrm{KCl}$, and 3 $\mathrm{mM} \mathrm{MgCl} 2$ and $10 \mathrm{U}$ RNAsin (Promega, WI, USA). Two microliters c-DNA from each sample was amplified in $10 \mu \mathrm{L}$ PCR reaction mixture. For semi-quantitative expression measurements, $200 \mathrm{ng}$ cDNA was diluted in five-fold increments (i.e. undiluted, $5 \times, 25 \times, 125 \times, 625 \times$ diluted). Eight microliters of each PCR sample was run on a $2 \%$ agarose gel containing $0.5 \mathrm{mg} / \mathrm{mL}$ ethidiumbromide (Invitrogen, Breda, The Netherlands). Positive bands were visualized and quantified with a Geldoc 1000 system using Multi-Analyst software (Bio-Rad, CA, USA). Values were normalized to $28 \mathrm{~S}$ rRNA expression.

\section{Real time PCR assays}

Real time PCR assays were performed in triplicates with qPCR mastermix for SYBR® Green I (Eurogentec, Seraing, Belgium) in 96-well optical plates with primer concentrations of $300 \mathrm{nM}$. Oligonucleotide sequences for Collagen Type I and II, aggrecan, BMP-2, -4, and -7, periostin, PTHrP, VEGF, 28S rRNA, GAPDH, Sox9, and HIF-1a (Supplemental table 2) were based on the gene sequences which were submitted to or obtained from GenBank (Supplemental table 1) using Primer Express 2.0 software (Applied Biosystems, Foster City, CA, USA). cDNA was used for the real time quantitative PCR reactions using an $A B I P R I S M \circledast 7700$ Sequence Detection System (Applied Biosystems, Foster City, CA, USA, user bulletin \#2). Serially diluted standard curves were 


\section{Chapter 3}

included to quantify the samples. PCR conditions were: 2 min at $50^{\circ} \mathrm{C}, 10 \mathrm{~min}$ at $95^{\circ} \mathrm{C}, 40$ cycles for $15 \mathrm{~s}$ at $95^{\circ} \mathrm{C}$, and $1 \mathrm{~min}$ at $60^{\circ} \mathrm{C}$ followed by a melt curve $15 \mathrm{~s}$ at $95^{\circ} \mathrm{C}$ ramp to $60^{\circ} \mathrm{C}$ for $1 \mathrm{~min}$, followed by $20 \mathrm{~s}$ at $95^{\circ} \mathrm{C}$. Data were analyzed using Sequence Detection Software version 1.7 (Applied Biosystems, Foster City, CA, USA). Values were normalized to 28S rRNA.

\section{Staining protocols}

Paraffin sections were deparaffinated, hydrated and stained with thionine for $10 \mathrm{~min}$. After washing and drying, coverslips were mounted using Entellan (Merck-Europe, Darmstad, Germany). For immunostaining, (paraffin-embedded) sections were deparaffinated and hydrated. Primary antibodies were diluted in PBS as follows: goat-polyclonal anti-BMP-2 (Santa Cruz, CA, USA, sc6895) 1:25; goat-polyclonal anti-BMP-4 (Santa Cruz, CA, USA, sc-6896) 1:12.5; goat-polyclonal anti BMP-7 (Santa Cruz, CA, USA, sc-9305) 1:800; goat-polyclonal anti-TGF- $\beta 1$ (R\&D Systems Inc, MN, USA) 1:30; mouse-monoclonal anti-periostin (5H8) 1:600 [28,29]; mouse monoclonal antiHIF-1a (Abcam Limited, Cambridge, UK, ab463) 1:50; and mouse monoclonal anti-Collagen Type II (II-II6B3, diluted 1:50; Developmental Studies Hybridoma Bank, University of lowa, lowa City, IA). Prior to the incubation with the goat-polyclonal anti-TGF- $\beta 1$, sections were placed in $250 \mathrm{~mL}$ Antigen Retrieval buffer (Dako Cytomation BV, Glostrup, Denmark) supplemented with $4.5 \mathrm{~mL}$ 0.1 Mcitric acid $+0.5 \mathrm{~mL} 0.1 \mathrm{MNa}$ citrate and incubated for $5 \mathrm{~min}$ in a microwave at $750 \mathrm{~W}$. After incubation with the primary antibody, sections were washed with PBS and incubated with an appropriate biotin-labeled secondary antibody. Subsequently, sections were washed with PBS and incubated with the avidin-biotin binding complex with horseradish peroxidase (HRP) or alkaline phosphatase (ALP) (Dako Cytomation BV, Glostrup, Denmark). Diaminobenzidine (DAB) (MerckEurope, Darmstad, Germany) was used as a substrate for HRP and Vectastain (Vector Laboratories, Burlingame, USA) for ALP. The sections were counterstained with hematoxylin dried and coverslipped.

\section{Statistical analysis}

Differences in mRNA levels were analyzed using the Mann-Whitney $U$ test with a Hochberg post hoc correction test. Statistical significance was assigned for $p<0.05$. 


\section{Results}

Dissection of periosteum induced reactive tissue (periosteal callus-PC) which demonstrated cartilaginous characteristics at 10 and 20 days postoperative (PO) and osteogenic at 40 days (PO) as previously published ${ }^{23}$. To validate the new osteo/ chondrogenic model, we mapped gene expression profiles during PC formation and differentiation and compared these to published data.

A

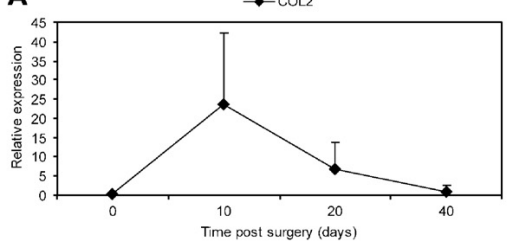

C

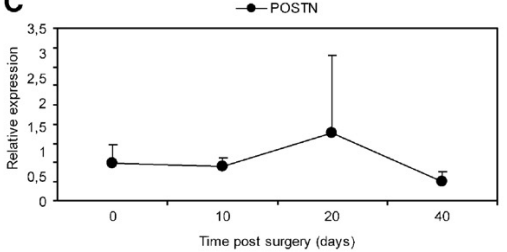

$\mathbf{E}$

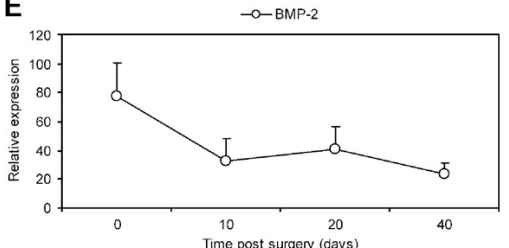

G
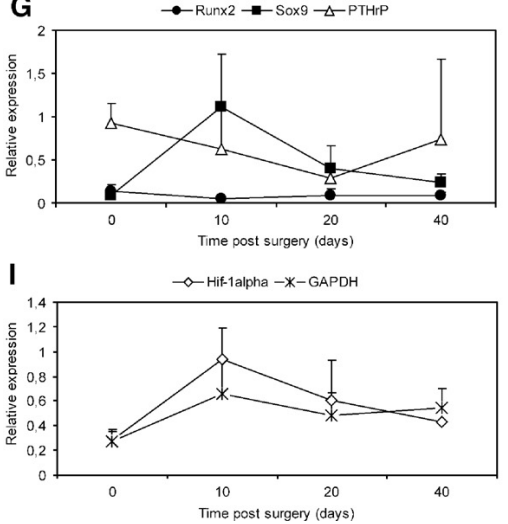

B

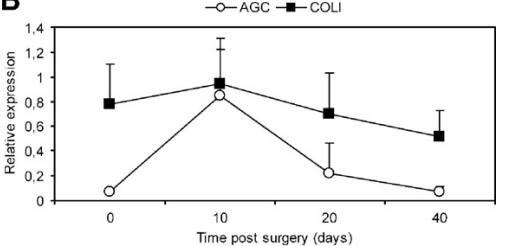

D

$\rightarrow$ TGF-beta

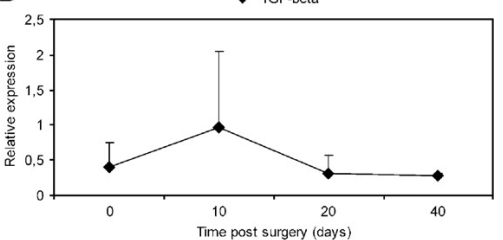

$\mathbf{F}$
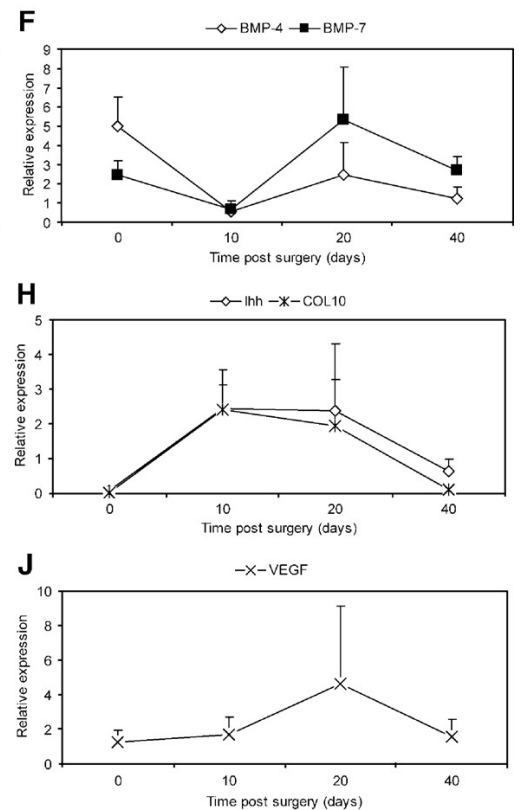

Fig. 1. Gene expression profiles throughout periosteal callus formation and differentiation ( $n=5$ per time point). mRNA expression level of Collagen Type II (COL2;A), aggrecan/AGC (B), Sox9 (F), COLX and Ihh (G), and HIF-1a (I) are significantly up-regulated during periosteal chondrogenesis $(t=10)$. No significant mRNAup- or down-regulation is observed for COLI (B), TGF- $\beta 1$ (C), Runx2 (F), and periostin/POSTN (H) during follow-up. BMP-2, -4 , and -7 are significantly downregulatedduring chondrogenesis (D, E). VEGF expression is up-regulated 20 days post-surgery (J). Quantitative RT-PCR expression measurements are normalized to $28 \mathrm{~S}$ ribosomal RNA. Relative COLX, Ihh, and TGF- $\beta 1$ mRNA levels were measured by semi-quantitative RT-PCR (see Materials and methods for details). Briefly,relevant marker gene cDNAs were either separately amplified or in combination with 28S rRNA. SD is indicated by error bars. 


\section{Matrix and adhesion molecules in PC}

As expected, in undamaged periosteum $(\mathrm{t}=0)$, Collagen Type II (COL2) expression was not detected. At 10 days $\mathrm{PO}$, a significant up regulation of this marker for chondrogenesis was clearly detectable (Fig. 1A). Forty days post-surgery, COL2 expression had almost returned to basal level (Fig. 1A). A comparable expression profile was seen for aggrecan (AGC). Collagen Type I (COLI) was not significantly up- or downregulated (Fig. 1B). Thionine staining and immunohistochemical detection of COL2 correlated nicely with mRNA expression levels: no AGC and COL2 expression was detectable in control periosteum ( $\mathrm{t}=0$; not shown). Ten and twenty days $\mathrm{PO}$, thionine staining indicated the presence of Glycosaminoglycans (GAG, i.a. AGC) and immunohistochemical detectable COL2 confirmed the expression of these proteins (Fig. 2A). Twenty days PO, ossification of the PC was first observed at the site attached to the tibial cortex (Fig. 2B). Forty days $\mathrm{PO}$, full ossification was observed and only remnants of cartilaginous matrix molecules were detected (Fig. 2C). mRNA levels of the adhesion molecule periostin (POSTN) were not significantly up- or down-regulated during follow-up (Fig. 1C). In good agreement with this, a positive immunohistochemical staining for POSTN was observed in periosteum. This adhesion molecule was found intra- and peri-cellular in both the cambium layer and the fibrous layer of periosteum $(t=0)(F i g .3 A)$. The matrix of the overlying fibrous layer remained positive during follow-up. At 10 days PO, POSTN was predominantly found in both hypertrophic and immature chondrocytes. The intensity of staining and number of POSTN positive cells were higher in chondrocytes located at then outside of the newly formed cartilage (Fig. 3C). Twenty days PO, only a few of the chondrocytes stained positive for POSTN. At 40 days $\mathrm{PO}$, the adhesion molecule could only be detected in some of the bone marrow cells (Fig. 3D).

\section{Growth factors in PC}

Throughout PC formation and differentiation, relative expression of TGF- $\beta 1$ did not significantly change (Fig. 1D). BMP-2, -4 , and -7 mRNA were down-regulated $(2.4 ; 8.9 ; 3.7$ decrease respectively) during the chondrogenic phase (Figs. 1E,F). Immunohistochemical staining of TGF$\beta 1$ confirmed protein presence in periosteum. TGF- $\beta 1$ was found predominantly peri-cellular in both the cambium layer and the fibrous layer of periosteum (not shown). BMP-2, -4 , and -7 proteins were not detected in normal periosteum. Ten days PO, BMP-2, -4 , and -7 were clearly detectable in the chondrocytes in the newly formed cartilaginous tissue; intense staining for all BMPs was specifically seen in the pre-hypertrophic chondrocytes. Fig. 4D only shows BMP-2 staining, a similar staining was observed for BMP-2, -4 , and -7 ; however, BMP-4 positive cells were detected less frequent and appeared to be more restricted to the hypertrophic zone (Figs. 4A, B). Ten days PO, TGF- $\beta 1$ was also found in both hypertrophic and mature chondrocytes and the overlying fibrous layer (not shown). Twenty days PO, BMP-2 and -4 could no longer be detected by 
immunohistochemical staining (Fig. 4C). The intensity of BMP-7 staining was lower; a positive staining was only found in some hypertrophic chondrocytes and some osteoblasts in the newly formed trabecular bone (not shown). At this follow-up, the staining intensity for TGF- $\beta 1$ also decreased and was only found in the newly formed trabecular bone. Forty days post-surgery, the newly formed bone marrow was positive for TGF- $\beta 1$ and BMP-2, -4 , and -7 (Fig. 4E, only BMP-2 is shown as example). Newly formed trabecular bone and bone marrow stained intensely for BMP-7; only a few cells were positive for BMP-2.

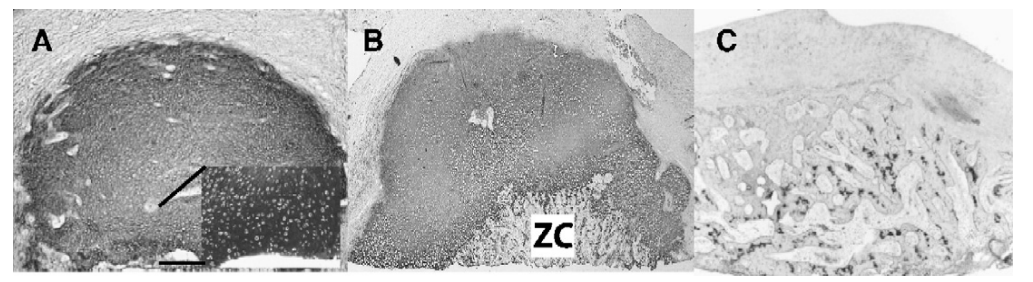

Fig. 2. Immunohistochemical detection of Collagen Type II in periosteal reactive tissue (callus); (A) 10 days post-surgery, this collagen is present in the newly formed cartilage (a thionine stained section is enlarged). (B) At 20 days postsurgery, the presence of Collagen Type II decreases at the zone of calcification (ZC and bone formation. (C) 40 days post-surgery, remnants of Collagen Type II are still visible (brown). Sections counterstained with hematoxylin. Original magnification $(A) \times 16$; $(B) \times 16$; $(C) \times 16$; insert in $(A) \times 100$.

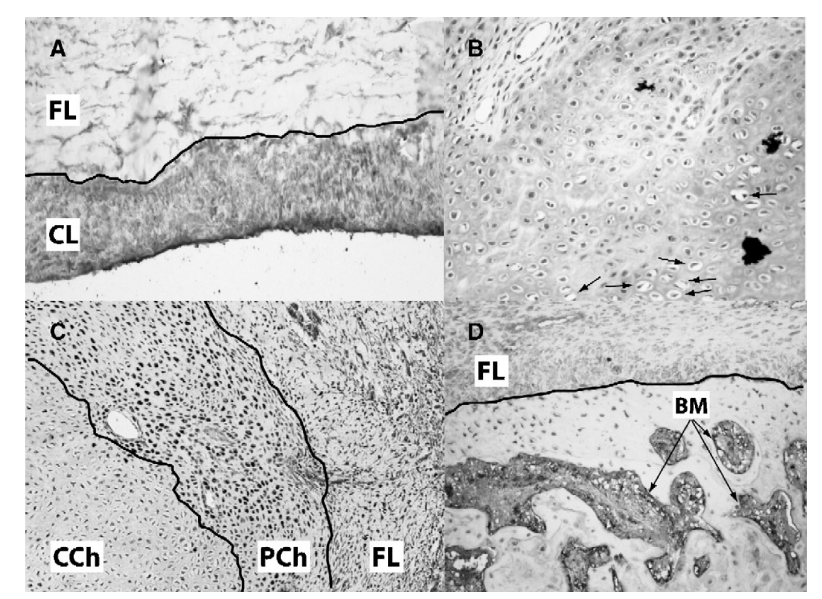

Fig. 3. Immunohistochemical detection of periostin/POSTN in periosteum. (A) POSTN is detected (brown color) especially in the cambium layer (CL) but is also found in the fibrous layer (FL) of periosteum. (C) 10 days post-surgery, the number of POSTN-positive cells is higher in the peripheral chondrocytes (PCh) compared to the chondrocytes localized centrally (CCh). (D) 40 days post-surgery, POSTN is present in the newly formed bone marrow (BM). (B) Immunohistochemical staining of HIF-1a shows its presence (red) in chondrocytes, 10 days post-surgery, no HIF-1a staining is found in hypertrophic chondrocytes (arrows). Original magnification (A) $\times 200 ;(B) \times 200 ;(C) \times 200 ;(D) \times 200$. 


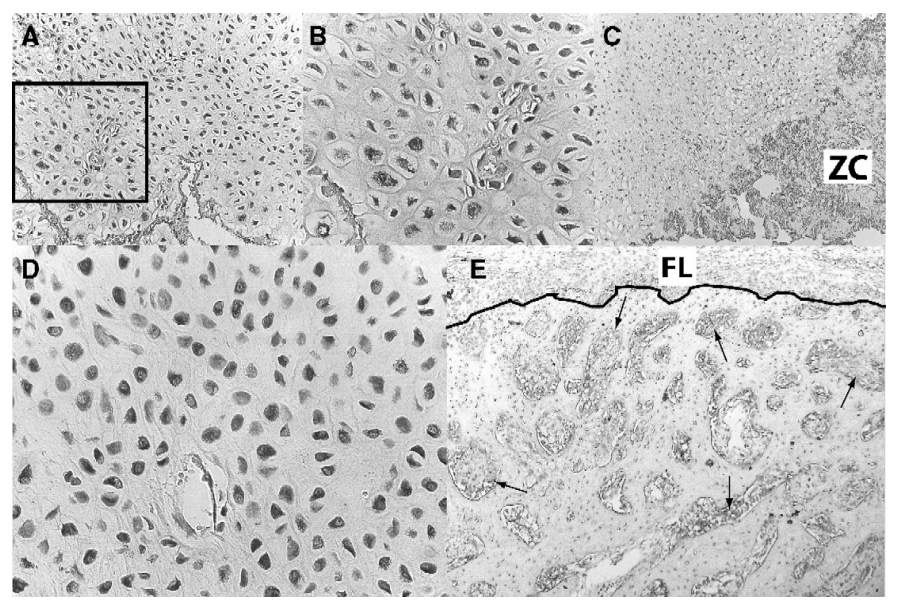

Fig. 4. Immunohistochemical detection of BMP-4 in periosteal callus; (A) 10 days post surgery, chondrocytes stain positive (red) for BMP-4. (B) is an enlargement of the box in (A). (C) 20 days post-surgery, BMP-4 is neither detected in chondrocytes nor in the zone of calcification $(Z C)$. Sections counterstained with hematoxylin. Immunohistochemical detection of BMP-2 in periosteal callus; (D) 10 days post-surgery, chondrocytes stain positive (red) for BMP-2. (E) 40 days post-surgery, BMP-2 is detected in the newly formed bone marrow (arrows), the overlying fibrous layer is indicated with FL. Original magnification $(A) \times 200 ;(B) \times 400 ;(C) \times 200 ;(D) \times 400 ;(E) \times 100$.

\section{Key lineage specific signaling and programming molecules in PC.}

The expression of the transcription factor Sox9 was significantly up-regulated (14 increase) at 10 days $P O$ compared to $t=0$ (Fig. 1G). At 20 and 40 days PO, the expression level of Sox9 gradually decreased. No significant up- or down-regulation was observed for Runx2 (Fig. 1G). At 40 days post-surgery, then mRNA expression level of this transcription factor returned to the same level as detected in periosteum ( $\mathrm{t}=0$; Fig. $1 \mathrm{G}$ ). In contrast to Parathyroid Hormone-related Protein (PTHrP) (Fig. 1G), Indian Hedgehog (Ihh) was not expressed in periosteum (Fig. 1H). During the chondrogenic phase $(\mathrm{t}=10)$ a significant up-regulation was seen for Ihh. At all later stages during osteogenesis, mRNA levels decreased for both these genes (Figs. 1G, H).

\section{Oxygenation and angiogenic marker genes}

A simultaneous increase in the mRNA expression level of GAPDH (2.6-fold increase) and a significant increase in expression level of HIF-1 $\alpha$ (3.4-fold increase) were detected at 10 days PO (Fig. 11). Immunohistochemical detection of HIF-1 $\alpha$ protein was reflected in the mRNA expression profile; HIF-1 $\alpha$ was only detectable in the chondrocytes present at 10 days post-surgery. Mature chondrocytes and early hypertrophic chondrocytes stained positive for HIF-1a (Fig. 3B). The protein was not detected in late hypertrophic chondrocytes (Fig. 3B). At 20 days PO, a few chondrocytes in the outer layer stained positive for HIF-1a. In periosteum and at 40 days PO, HIF$1 \alpha$ protein could not be detected by immunohistochemical staining. VEGF mRNA levels expression increased 20 days post-surgery (2.7-fold increase) (Fig. $1 \mathrm{~J}$ ). 


\section{Discussion}

We here report on a first analysis of lineage specific marker gene expression in a novel in vivo model for osteo/chondrogenesis in full-grown animals. The model makes use of experimentally induced local damage to the periosteum. The damaged periosteum responds by callus formation; within this periosteal callus (PC), MSC-like activity is mobilized, which subsequently supports and recapitulates several well-described stages of chondrogenesis and osteogenesis. During the chondrogenic phase of PC formation, we find that cells of the cambium layer first commit to a chondrogenic cell fate; mRNA of the transcription factors Sox9, Ihh, is up-regulated and cartilage matrix molecules like aggrecan and Collagen Types II and $X$ are produced. This stage is followed by chondrocyte hypertrophy and osteogenesis as indicated by extensive calcification of surrounding matrix (to independently verify these results, we partially repeated expression analysis of matrix molecules throughout differentiation, as reported in one of our earlier studies ${ }^{23}$ ). Thus far, whole embryos or the growth plate are the models of choice for study of endochondral bone formation in vivo ${ }^{5,6,20}$. This novel concept represents a relevant pre-clinical animal model, which thus enables us to study periosteum derived endochondral bone formation in vivo in the context of physiologically relevant signaling. Cartilage is known to lack vascularization, and oxygenation probably decreases during this first step of PC formation ${ }^{9}$. In addition, because of metabolic activity and concomitant oxygen consumption, oxygen tension is expected to be low in the direct vicinity of cells ${ }^{30}$. These factors combined most likely contribute to reduced oxygen tension in our model system. The increase in mRNA expression levels of GAPDH and HIF-1 $\alpha$ and the presence of HIF-1 $\alpha$ protein are indicative of a response to a change in oxygenation, and a metabolic shift indicative of adaptation to hypoxia. Under normoxia, the HIF-1 $\alpha$ subunit is rapidly degraded via a concerted action of prolyl hydroxylases, which hydroxylate HIF-1 $\alpha$, and the von Hippel-Lindau tumor suppressor gene product VHL, which ubiquitylates HIF-1a and thereby targets it for proteasomal degradation. Oxygen deprivation blocks hydroxylation and thereby stabilizes HIF-1a. These processes play an important role in chondrocyte growth arrest and survival under hypoxia ${ }^{31,31}$. During the chondrogenic phase, many essential factors are activated by HIF-1a-dependent

mechanisms ${ }^{8,14,33-35}$. HIF-1 $\alpha$ plays an important role in the "Pasteur effect" which includes decreased oxidative phosphorylation and an increase in glycolysis ${ }^{36}$. Since glycolytic ATP production is significantly less efficient per molecule of glucose compared to that by oxidative phosphorylation, glycolytic metabolism is up-regulated to sustain free ATP levels in the hypoxic cell. In good agreement with published data in other model systems, our PC formation model shows a concomitant increase of HIF-1 $\alpha$ and GAPDH mRNA levels, one of the key enzymes in glycolysis and a known HIF-1 $\alpha$ target ${ }^{36}$. Interestingly, while the mRNA expression levels of BMP-2, -4 , and -7 were significantly decreased during chondrogenesis, these growth factors were readily detectable by immunohistochemical staining at 10 and 40 days PO. Reports on BMP expression levels during chondrogenesis are inconsistent: Yaoita et al. report an unaffected expression of BMPs after inducing a fracture, while Kloen et al. report a positive staining of BMPs in human 


\section{Chapter 3}

callus ${ }^{37-38}$. Others report a rapid upregulation of BMP-2, only $24 \mathrm{~h}$ after surgical release of the periosteum ${ }^{39}$. Uusitalo et al. reported an inducing effect on periosteal endochondral callus formation in mice upon adenovirus-mediated gene delivery of BMP-2 ${ }^{7}$. The downregulation of BMP-2 at the RNA level we observe is part of a genetic reprogramming controlled by autologous regulatory sequences, which may be absent in adenoviral vectors. Factors like these most likely contribute to fine-tuning of the in vivo response and further substantiate the complexity of BMP-2 regulation during chondrogenesis. The results of the study presented herein indicate that, throughout periosteal callus formation and osteochondrogenic differentiation, BMP expression is regulated both by transcriptional and post-transcriptional mechanisms. Increased association with polysomes during conditions of cell stress (e.g. hypoxia) has recently been shown to result in preferential translation of select mRNAs ${ }^{40}$. It is tempting to speculate that such mechanisms act on BMP and other mRNAs. The exact mechanism of translational regulation of cartilage matrix molecules, BMPs, Sox9, Ihh, PTHrP, and periostin during chondrogenesis under conditions of lowered oxygen availability remains unknown and is subject of further study in our laboratories. PTHrP expression was found in periosteum. In the growth plate, this molecule diffuses to sites of hypertrophic differentiation of chondrocytes to inhibit this process ${ }^{2,41}$. Ihh was not expressed in periosteum, but its expression was markedly increased during chondrogenesis at 10 days, which is an indicator of hypertrophic chondrocyte differentiation ${ }^{41}$. Combined, the presence of both Ihh and PTHrP, the sites at which they are co-expressed, and the timing of their expression are highly indicative of a function for the Ihh/PTHrP interplay in periosteal callus formation equivalent to their proposed role in growth plate models ${ }^{41}$. In contrast to other reports ${ }^{14,19}$, in which POSTN mRNA was not detected in chondrocytes, the present study shows that POSTN protein was detectable in mature chondrocytes. POSTN is an extracellular matrix/adhesion molecule ${ }^{16}$, however, its exact function during osteo- and chondrogenesis is unclear. Our immunolocalization studies confirmed this notion, in that POSTN is also detected in the cartilaginous matrix around POSTN-positive chondrocytes. POSTN expression, both at the mRNA and protein level, is increased during fracture healing possibly to enhance the recruitment and adhesion of chondro- and osteo-progenitors from essential sources such as bone marrow and blood ${ }^{19}$. The higher number of peripherally located POSTN-positive cells in the newly formed cartilage is consistent with this idea. In line with our observation that both TGF- $\beta$ and POSTN have similar mRNA expression profile, several studies report a TGF- $\beta$ dependency of POSTN regulation ${ }^{42}$. POSTN expression is regulated downstream of cellular stress responses, including hypoxia, i.e. during vascular remodeling in different systems 13,42,43. Ectopic POSTN expression in several models increases adhesion, migration, and invasiveness ${ }^{44,45}$. These properties may relate to its proposed role in epithelial-mesenchymal transition ${ }^{44}$. As a ligand for alpha-V/beta-3 and alpha-V/beta-5, POSTN mediates that growth promoting and angiogenic signaling in experimental cancers ${ }^{46,47}$. We do not find significant upregulation of POSTN at the mRNA level, yet protein detection suggests that POSTN may be regulated in a cell type specific manner during chondrogenesis in our model. The combined above 
data suggest that POSTN may mediate comparable processes during chondrogenesis and endochondral bone formation. Whether regulation of POSTN includes a selective translational component, for instance in PC, awaits further analysis. In conclusion, in our periosteal callus formation model, the sequential steps of endochondral bone development are recapitulated and we were able to describe these processes in some cellular and molecular detail: presumptive chondrocytes undergo well-ordered and controlled phases of proliferation, hypertrophic differentiation, mineralization of the surrounding matrix, and finally the cartilaginous tissue is replaced by bone. We have provided evidence that HIF-1 $\alpha$ is activated during the chondrogenic phase of periosteal callus formation, which suggests that conditions during callus formation and differentiation are, at least transiently, hypoxic. Interestingly, expression of BMPs appears to be regulated at a posttranscriptionallevel during these processes.

\section{Acknowledgments}

This study was supported by grant BTS00021 from Senter a ZonMw VIDI grant 016.046.362 and by grant LLP14 of the Dutch Reumafonds. The periostin antibodies were kindly provided by Lan Bo Chen, Sasaki Hidefumi, and Meiru Dai (Harvard Medical School and University of Pittsburgh). The monoclonal antibodies developed by T.F. Linsenmayer et al. (IIII6B3) were obtained from the Developmental Studies Hybridoma Bank under auspices of the NICHD and maintained by the Department of Biological Sciences of the University of lowa (lowa City, IA). The help of May Bost, Monique de Jong, Joyce Suyk, and Petra Dijkstra from the Central animal facility in the animal experiments is gratefully acknowledged. 


\section{References}

1. Zhao Q, Eberspaecher $\mathrm{H}$, Lefebvre V, De Crombrugghe B. Parallel expression of Sox9 and Col2a1 in cells undergoing chondrogenesis. Dev Dyn 1997;209:377-86.

2. Yoon BS, Lyons KM. Multiple functions of BMPs in chondrogenesis. J Cell Biochem 2004;93:93103.

3. Akiyama H, Chaboissier MC, Martin JF, Schedl A, de Crombrugghe B. The transcription factor Sox9 has essential roles in successive steps of the chondrocyte differentiation pathway and is required for expression of Sox5 and Sox6. Genes Dev 2002;16:2813-28.

4. Bridgewater LC,Walker MD, Miller GC, Ellison TA, Holsinger LD, Potter JL, et al. Adjacent DNA sequences modulate Sox9 transcriptional activation at paired Sox sites in three chondrocyte-specific enhancer elements. Nucleic Acids Res 2003;31:1541-53.

5. Lefebvre V, Li P, de Crombrugghe B. A new long form of Sox5 (L-Sox5), Sox6 and Sox9 are coexpressed in chondrogenesis and cooperatively activate the type II collagen gene. EMBO J 1998;17:5718-33.

6. Lefebvre V, Behringer RR, de Crombrugghe B. L-Sox5, Sox6 and Sox9 control essential steps of the chondrocyte differentiation pathway. Osteoarthr Cartil 2001;9(Suppl A):S69-75.

7. Uusitalo H, Hiltunen A, Ahonen M, Kahari VM, Aro H, Vuorio E. Induction of periosteal callus formation by bone morphogenetic protein-2 employing adenovirus-mediated gene delivery. Matrix Biol 2001;20:123-7.

8. Robins JC, Akeno N, Mukherjee A, Dalal RR, Aronow BJ, Koopman P, et al. Hypoxia induces chondrocyte-specific gene expression in mesenchymal cells in association with transcriptional activation of Sox9. Bone 2005;37:313-22.

9. Schipani E. Hypoxia and HIF-1alpha in chondrogenesis. Semin Cell Dev Biol 2005;16:539-46.

10. Gersbach CA, Byers BA, Pavlath GK, Garcia AJ. Runx2/Cbfa1 stimulates transdifferentiation of primary skeletal myoblasts into a mineralizing osteoblastic phenotype. Exp Cell Res 2004;300:40617.

11. Otto F, Thornell AP, Crompton T, Denzel A, Gilmour KC, Rosewell IR, et al. Cbfa1, a candidate gene for cleidocranial dysplasia syndrome, is essential for osteoblast differentiation and bone development. Cell 1997;89:765-71.

12. Xiao ZS, Simpson LG, Quarles LD. IRES-dependent translational control of Cbfa1/Runx2 expression. J Cell Biochem 2003;88:493-505.

13. Li P, Oparil S, Feng W, Chen YF. Hypoxia-responsive growth factors upregulate periostin and osteopontin expression via distinct signalling pathways in rat pulmonary arterial smooth muscle cells. J Appl Physiol 2004;97:1550-8 (discussion 1549).

14. Horiuchi K, Amizuka N, Takeshita S, Takamatsu H, Katsuura M, Ozawa H, et al. Identification and characterization of a novel protein, periostin, with restricted expression to periosteum and periodontal ligament and increased expression by transforming growth factor beta. J Bone Miner Res 1999;14:1239-49.

15. LeBaron RG, Bezverkov KI, Zimber MP, Pavelec R, Skonier J, Purchio AF. Beta IG-H3, a novel secretory protein inducible by transforming growth factor-beta, is present in normal skin and promotes the adhesion and spreading of dermal fibroblasts in vitro. J Invest Dermatol 1995;104:8449.

16. Takeshita S, Kikuno R, Tezuka K, Amann E. Osteoblast-specific factor 2: cloning of a putative bone adhesion protein with homology with the insect protein fasciclin I. Biochem J 1993;294(Pt 1):271-8.

17. Wilde J, Terai K, Yokozeki M, Hiura K, Horiuchi K, Kudo A, et al. Compressive mechanical stress increases periostin mRNA expression in the periochondrium. J Bone Miner Res 2000;15:S348.

18. Wilde J, Yokozeki M, Terai K, Kudo A, Moriyama K. The divergent expression of periostin mRNA in the periodontal ligament during experimental tooth movement. Cell Tissue Res 2003;312:345-51.

19. Nakazawa T, Nakajima A, Seki N, Okawa A, Kato M, Moriya H, et al. Gene expression of periostin in the early stage of fracture healing detected by cDNA microarray analysis. J Orthop Res 2004;22:520-5.

20. Lefebvre V, Huang W, Harley VR, Goodfellow PN, de Crombrugghe B. SOX9 is a potent activator of the chondrocyte-specific enhancer of the pro alpha1(II) collagen gene. Mol Cell Biol 1997;17:233646.

21. Einhorn TA. The science of fracture healing. J Orthop Trauma 2005;19: S4-6.

22. Bostrom MP, Lane JM, Berberian WS, Missri AA, Tomin E,Weiland A, et al. Immunolocalization and expression of bone morphogenetic proteins 2 and 4 in fracture healing. J Orthop Res 1995;13:35767.

23. Emans PJ, Surtel DA, Frings EJ, Bulstra SK, Kuijer R. In vivo generation of cartilage from periosteum. Tissue Eng 2005;11:369-77.

24. Zohar R, Sodek J, McCulloch CA. Characterization of stromal progenitor cells enriched by flow cytometry. Blood 1997;90:3471-81. 
25. Ghilzon R, McCulloch CA, Zohar R. Stromal mesenchymal progenitor cells. Leuk Lymphoma 1999;32:211-21.

26. O'Driscoll SW, Recklies AD, Poole AR. Chondrogenesis in periosteal explants. An organ culture model for in vitro study. J Bone Jt Surg, Am 1994;76:1042-51.

27. Sanyal A, Sarkar G, Saris DB, Fitzsimmons JS, Bolander ME, O'Driscoll SW. Initial evidence for the involvement of bone morphogenetic protein-2 early during periosteal chondrogenesis. J Orthop Res 1999;17:926-34.

28. Sasaki H, Roberts J, Lykins D, Fujii Y, Auclair D, Chen LB. Novel chemiluminescence assay for serum periostin levels in women with preeclampsia and in normotensive pregnant women. Am J Obstet Gynecol 2002;186:103-8.

29. Sasaki H, Yu CY, Dai M, Tam C, Loda M, Auclair D, et al. Elevated serum periostin levels in patients with bone metastases from breast but not lung cancer. Breast Cancer Res Treat 2003;77:245-52.

30. Metzen E, Wolff M, Fandrey J, Jelkmann W. Pericellular PO2 and O2 consumption in monolayer cell cultures. Respir Physiol 1995;100:101-6.

31. Schipani E, Ryan HE, Didrickson S, Kobayashi T, Knight M, Johnson RS. Hypoxia in cartilage: HIF1alpha is essential for chondrocyte growth arrest and survival. Genes Dev 2001;15:2865-76.

32. Lee JW, Bae SH, Jeong JW, Kim SH, Kim KW. Hypoxia-inducible factor (HIF-1)alpha: its protein stability and biological functions. Exp Mol Med 2004;36:1-12.

33. Minchenko A, Bauer T, Salceda S, Caro J. Hypoxic stimulation of vascular endothelial growth factor expression in vitro and in vivo. Lab Invest 1994;71:374-9.

34. Levy NS, Chung S, Furneaux H, Levy AP. Hypoxic stabilization of vascular endothelial growth factor mRNA by the RNA-binding protein HuR. J Biol Chem 1998;273:6417-23.

35. Lu S, Gu X, Hoestje S, Epner DE. Identification of an additional hypoxia responsive element in the glyceraldehyde-3-phosphate dehydrogenase gene promoter. Biochim Biophys Acta 2002;1574:1526.

36. Seagroves TN, Ryan HE, Lu H, Wouters BG, Knapp M, Thibault $P$, et al. Transcription factor HIF-1 is a necessary mediator of the Pasteur effect in mammalian cells. Mol Cell Biol 2001;21:3436-44.

37. Yaoita H, Orimo H, Shirai Y, Shimada T. Expression of bone morphogenetic proteins and rat distalless homolog genes following rat femoral fracture. J Bone Miner Metab 2000;18:63-70.

38. Kloen P, Di Paola M, Borens O, Richmond J, Perino G, Helfet DL, et al. BMP signaling components are expressed in human fracture callus. Bone 2003;33:362-71.

39. Simon TM, Van Sickle DC, Kunishima DH, Jackson DW. Cambium cell stimulation from surgical release of the periosteum. J Orthop Res 2003;21:470-80.

40. Koritzinsky M, Magagnin MG, van den Beucken T, Seigneuric R, Savelkouls K, Dostie J, et al. Gene expression during acute and prolonged hypoxia is regulated by distinct mechanisms of translational control. EMBO J 2006;25:1114-25.

41. Kronenberg $\mathrm{HM}$, Chung $\mathrm{U}$. The parathyroid hormone-related protein and Indian hedgehog feedback loop in the growth plate. Novartis Found Symp 2001;232:144-52 (discussion 152-7).

42. Chen YF, Feng JA, Li P, Xing D, Ambalavanan N, Oparil S. Atrial natriuretic peptide-dependent modulation of hypoxia-induced pulmonary vascular remodeling. Life Sci 2006:1357-65.

43. Li G, Oparil S, Sanders JM, Zhang L, Dai M, Chen LB, et al. Phosphatidylinositol-3-kinase signaling mediates vascular smooth P.J. Emans et al. / Bone 40 (2007) 409-418 417 muscle cell expression of periostin in vivo and in vitro. Atherosclerosis 2005 [Electronic publication ahead of print].

44. Yan W, Shao R. Transduction of a mesenchyme-specific gene periostin into 293T cells induces cell invasive activity through epithelial-mesenchymal transformation. J Biol Chem 2006:19700-8.

45. Kii I, Amizuka N, Minqi L, Kitajima S, Saga Y, Kudo A. Periostin is an extracellular matrix protein required for eruption of incisors in mice. Biochem Biophys Res Commun 2006;342:766-72.

46. Shao R, Bao S, Bai X, Blanchette C, Anderson RM, Dang T, et al. Acquired expression of periostin by human breast cancers promotes tumor angiogenesis through up-regulation of vascular endothelial growth factor receptor 2 expression. Mol Cell Biol 2004;24:3992-4003. [47] Gillan L, Matei D, Fishman DA, Gerbin CS, Karlan BY, Chang DD. Periostin secreted by epithelial ovarian carcinoma is a ligand for alpha(V) beta(3) and alpha(V)beta(5) integrins and promotes cell motility. Cancer Res 2002;62:5358-64. 


\section{Supplemental Tables}

Table 1

Semi-quantitative RT-PCR primer sets

\begin{tabular}{|c|c|c|c|c|c|}
\hline cDNA & Size & Primer sequence & & GenBank accession & Homology with human \\
\hline \multirow[t]{2}{*}{ COLI } & 511 & 5'-GCACCCATGGACATTGGAGGG-3' & $\mathrm{S}$ & AY633663 & $92 \%$ \\
\hline & & 5'-GACACGGAGTAGCACCATCG-3' & AS & & \\
\hline \multirow[t]{2}{*}{ COLX } & 329 & 5'-ACAGGAATGCCTGTGTCTGCTTTT-3' & $\mathrm{S}$ & AY598937 & $91 \%$ \\
\hline & & 5'-TTGGGAAGCTGGAGCCACACCTGGT-3' & AS & & \\
\hline \multirow[t]{2}{*}{ TGF- $\beta_{1}$} & 271 & 5'-CGGCAGCTGTACATTGACTT-3' & $\mathrm{S}$ & AF000133 & $\mathrm{nc}$ \\
\hline & & 5'-AGCGCACGATCATGTTGGAC-3' & AS & & \\
\hline \multirow[t]{2}{*}{ Sox 9} & 553 & 5'-TGAATCTCCTGGACCCCTTC-3' & $\mathrm{S}$ & AY598935 & $96 \%$ \\
\hline & & 5'-CCGTTCTTCACCGACTTCCT-3' & AS & & \\
\hline \multirow[t]{2}{*}{ Runx2 } & 280 & 5'-TGATGACACTGCCACCTGTG-3' & $\mathrm{S}$ & AY598934 & $99 \%$ \\
\hline & & 5'-ACTCTGGCTTTGGGAAGAGC-3' & AS & & \\
\hline \multirow[t]{2}{*}{ POSTN } & 293 & 5'-CCACGAGGTGTCCTAGAAAGG-3' & $\mathrm{S}$ & AY598936 & $91 \%$ \\
\hline & & 5'-TGTTTTCCAGCCAGCTCAAT-3' & AS & & \\
\hline \multirow[t]{2}{*}{ Ihh } & 318 & 5'-TTCCAGGTCATCGAGACTCA-3' & $\mathrm{S}$ & AY598933 & $90 \%$ \\
\hline & & 5'-CCAGAAGGCCAACTGAGC-3' & AS & & \\
\hline \multirow[t]{2}{*}{$28 \mathrm{~S}$ rRNA } & 188 & 5'-GGGTTTAGACCGTCGTGAGA-3' & $\mathrm{S}$ & AF460236 & $\mathrm{nc}$ \\
\hline & & 5'-GCGGGATTCTGACTTAGAGG-3' & AS & & \\
\hline
\end{tabular}

Table 2

Real time RT PCR primer sets

\begin{tabular}{|c|c|c|c|c|}
\hline cDNA & Size & Primer sequence & & GenBank accession \\
\hline COLI & 81 & $5^{\prime}$-CTGACTGGAAGAGCGGAGAGTAC- $3^{\prime}$ & $\mathrm{S}$ & AY633663 \\
\hline COLII & 84 & $\begin{array}{l}\text { 5'-TGGGTGTTCTATTTATTTATTGTCTTCCT-3' } \\
\text { 5'-GCGTTGGACTCACACCAGTTAGT-3' }\end{array}$ & S & $\mathrm{S} 83370$ \\
\hline BMP-2 & 76 & $\begin{array}{l}\text { 5'-AGAAAAGCGTCAAGCGAAACA-3' } \\
\text { 5'-GTCCACGTACAAAGGGTGTCTCT-3' }\end{array}$ & $\begin{array}{l}\mathrm{S} \\
\mathrm{AS}\end{array}$ & $\mathrm{AF} 041421$ \\
\hline BMP-4 & 66 & $\begin{array}{l}\text { 5'-ACCCGCAGCCTAGCAAGA-3' } \\
\text { 5'-CTGACTGGAGCCGGTAAAGATC-3' }\end{array}$ & AS & $\mathrm{AF} 042497$ \\
\hline BMP-7 & 79 & $\begin{array}{l}\text { 5'-GCAACCACTGGGTGGTCAAC-3' } \\
\text { 5'-TGATGCTCT GCCCATCCA-3' }\end{array}$ & $\begin{array}{l}\text { S } \\
\text { AS }\end{array}$ & AF413111 \\
\hline Sox 9 & 79 & $\begin{array}{l}\text { 5'-AGTACCCGCACCTGCACAAC-3' } \\
\text { 5'-CGCTTCTCGCTCTCGTTCAG-3' }\end{array}$ & $\begin{array}{l}\text { S } \\
\text { AS }\end{array}$ & AY598935 \\
\hline Runx2 & 70 & $\begin{array}{l}\text { 5'-TGATGACACTGCCACCTCTGA-3' } \\
\text { 5'-GCACCTGCCTGGCTCTTCT-3' }^{\prime}\end{array}$ & $\begin{array}{l}\text { S } \\
\text { AS }\end{array}$ & AY598934 \\
\hline POSTN & 87 & $\begin{array}{l}\text { 5'-AATACTCTCCAGTGTTCTGAGGCTATT-3' } \\
\text { 5'-CAGCTTATCTCAATTGTGTTTCCTT-3' }\end{array}$ & $\begin{array}{l}\text { S } \\
\text { AS }\end{array}$ & AY598936 \\
\hline $\mathrm{HIF}-1 \alpha$ & 79 & $\begin{array}{l}\text { 5'-GGATATAAATTTGGCAATGCTTCCAT-3' } \\
\text { 5'-GAGTGCAGGGTCAGCACTACTTC-3' }\end{array}$ & AS & AY 273790 \\
\hline VEGF & 73 & $\begin{array}{l}\text { 5'-ACGAAGTGGTGAAGTTCATGGAA-3' } \\
\text { 5'-AAGATGTCCACCAAGGTCTCGAT-3' }\end{array}$ & $\begin{array}{l}\text { S } \\
\text { AS }\end{array}$ & AY 196796 \\
\hline GAPDH & 107 & $\begin{array}{l}\text { 5'-ACTTTGTGAAGCTCATTTCCTGGTA-3' } \\
\text { 5'-GTGGTTTGAGGGCTCTTACTCCTT-3' }\end{array}$ & $\begin{array}{l}\text { S } \\
\text { AS }\end{array}$ & L 23961 \\
\hline 28S rRNA & 73 & $\begin{array}{l}\text { 5'-GCCATGGTAATCCTGCTCAGTAC-3' } \\
\text { 5'-GCTCCTCAGCCAAGCACATAC-3' }\end{array}$ & $\begin{array}{l}\text { S } \\
\text { AS }\end{array}$ & AF 460236 \\
\hline
\end{tabular}

Abbreviations: AS, antisense; S, sense. 


\section{Chapter 4}

\section{The immediate early gene EGR1 controls epigenetic reprogramming through Polycomb Group proteins during chondrogenesis.}

Frank Spaapen ${ }^{1}$, Guus van den Akker, ${ }^{1,4}$ Marjolein Caron ${ }^{2,4}$, Peggy Prickaerts ${ }^{1,4}$, Vivian Dahlmans ${ }^{1}$, Don A.M. Surtel ${ }^{2}$, Yvette Paulis ${ }^{1}$, Finja Schweizer ${ }^{1}$, Celine Rofel ${ }^{1}$, Tim Welting ${ }^{2}$, Lars Eijssen ${ }^{3}$, Jan Willem Voncken ${ }^{1}$.

1 Department of Molecular Genetics, GROW - School for Oncology and Developmental Biology, Maastricht University, Maastricht, The Netherlands

2 Department of Orthopaedics, GROW - School for Oncology and Developmental Biology,

Maastricht University, Maastricht, The Netherlands

3 Department of Bioinformatics - BiGCaT; NUTRIM School for Nutrition Research Maastricht University, Maastricht, The Netherlands

4 authors with equal contribution

* Corresponding author: J.W. Voncken (w.voncken@gen.unimaas.nl)

In preparation 


\begin{abstract}
Initiation of and progression through chondrogenesis is driven by changes in the cellular microenvironment. For instance, Insulin Receptor signaling is known to mobilize resting mesenchymal stem cells and to initiate a complex, step-wise chondrogenic differentiation program in vivo and in vitro. Differentiation requires coordinated transcriptomic reprofiling and increased progenitor proliferation, both processes require chromatin remodeling. The nature of early molecular responses that relay differentiation signals to chromatin is poorly understood. We here show that immediate early genes are rapidly and transiently induced in response to a changed cellular microenvironment. Functional ablation of Egr1 severely affects induction of genes required for proper differentiation. In addition, differentiating cells accumulate DNA damage, activate a DNA damage response and induce a senescence-like response, which terminates in global histone hypermethylation. Novel molecular connections with Polycomb Group function identified in this report begin to explain the replicative arrest. We here for the first time describe an important role for Egr1 in early epigenomic reprogramming, exceeding its classical role as transcription factor, to accommodate early gene-environment interactions in chondrogenesis.
\end{abstract}




\section{Introduction}

Differentiation requires orchestration of numerous parallel cellular responses, processes and altered physiological states associated with the novel cell fate. Such changes are often induced by environmental cues (i.a. soluble factors, cell-cell contacts), which are signaled to the nucleus and translated into spatio-temporal re-profiling of gene expression. As coding and non-coding genes are 'packed' in chromatin, access to and activation of genes during differentiation is subject to important epigenetic regulation, including covalent modifications to histone proteins and chromatin structure remodeling. Post-translational modifications on histone tails serve to recruit either transcriptional activators or repressors and nucleosome remodeling machineries, and as such constitute an epigenetic register of expression potential ${ }^{1-3}$. Recent studies have provided important insights in epigenomic changes that accompany lineage commitment of ES cells. ES cells respond rapidly to changes in the micro environment: as ES cells are induced to differentiate, their epigenome is remodeled such that lineage-related genetic programs are selectively activated, whereas non-lineage relevant genes are progressively inactivated. Part of these epigenetic alterations includes post-translational modifications at amino-terminal tails of histones, which are closely associated with DNA, i.e. genes, as they are the major constituents of nucleosomes. Interestingly, many key developmental regulator genes in pluripotent ES cells appear bivalently marked: they carry both 'activating' (e.g. H3K4me3; histone $\mathrm{H} 3$, lysine 4, trimethyl) as well as 'inactivating' (e.g. H3K27me3) epigenetic marks in the chromatin surrounding transcription start sites. This bivalent marking switches to monovalency as the differentiation program proceeds ${ }^{4}$. These observations suggest that although common epigenetic principles exist to initiate cell fate decisions, specific cellular cues are integrated that eventually allow successful lineage demarcation.

Microenvironmental factors play an important role in chondrogenesis. As chondrogenic progenitors move away from the growth plate, their microenvironment changes constantly. These changes include soluble growth and differentiation factors, hormones, oxygen tension, cell-cell and cell ECM contacts ${ }^{5-8}$. Cells respond to these changes in the microenvironment by altering their gene expression profiles, which in turn are accompanied by locally changing in the epigenome. An important open question concerning the most primary choices that are made by undifferentiated cells is: What connects these environmental cues to chromatin? Which signaling factors are involved in early epigenomic remodeling and, hence, in differentiation? The Polycomb repressive Complex (PRC) proteins Ezh2 and Bmi1 are important factors in cell fate determination in that they provide cells with an epigenetic memory function: Ezh2 is a histone lysine methyl transferase (HKMT) in Polycomb repressive complex 2 (PRC2), which writes the H3K27me3 mark ${ }^{9}$. Bmi1 is part of Polycomb repressive complex 1 (PRC1), which is recruited to H3K27me3 marks and maintains transcriptionally repressed states ${ }^{10-12}$. As such, PRCs are recognized to sustain lineage commitment in the context of development. We and others have recently shown that Polycomb 


\section{Chapter 4}

function is regulated by post-translational modifications (reviewed by HN JWV; in press), suggesting a link between cell signaling and epigenetic re-programming involving PRC. The observation that PRC LOF mutant mice often display abnormal skeletogenesis indicates a potential link to endochondral ossification (i.e. formation of an ossified skeleton from a cartilagenous scaffold) ${ }^{13-16}$.

We here study chondrogenesis as a function of environmental changes. On their way to becoming terminally differentiated chondrocytes, chondrogenic progenitor cells in vivo undergo a welldescribed sequential series of events at the cell biology level: initially resting growth plate stem cells undergo a transit replicative burst. In vivo, this rapid progenitor expansion in the so called proliferative zone is a distinctive feature of developing cartilage. During endochondral ossification, as cells move away from the growth plate, they differentiate, become hypertrophic and are replaced by bone tissue ${ }^{17,18}$. Chondrogenesis is controlled by numerous well-described environmental and endocrinoid factors ${ }^{6-8,17-19}$. Per example, signaling through the insulin receptor has been intensively studied because of its mobilizing effect on resting stem cells and stimulatory effects on cells in the proliferative zone ${ }^{20}$. ATDC5 cells were originally identified as stem cells of mesenchymal origin that harbor chondrogenic potential ${ }^{21}$. The ATDC5 model uniquely combines a number of relevant elements. Chondrogenic differentiation of ATDC5 cells reiterates numerous micro-environmental changes that accompany dynamic and strictly timed transcriptomic re-profiling observed during embryogenesis ${ }^{21-23}$. In addition, proliferation is rapidly upregulated early in differentiation ${ }^{21}$. Hence ATDC5 cells represent an excellent model to study gene-environment interaction at the epigenetic level.

The rapid activation of immediate early genes (IEG) denotes the first line of cellular responses to environmental stimuli. Expression of IEG encoded proteins precedes de novo synthesis of other proteins; as such IEGs represent an important gateway to genomic responses and physiological adaptation. For instance, FOS, JUN and EGR1 (KROX24/NGFR1) induction is a well-described feature of neurotransmission ${ }^{24,25}$. In the context of cancer, EGR1 acts either oncogenic or tumorsuppressive ${ }^{26}$, hence, in some cancers it is overexpressed, in others egr1 is deleted.

Not much is known about the role of EGR1 in chondrogenesis. We here report that IEG family members (i.a. cFos, cJun, EGR) are rapidly induced in response to insulin signaling. We show that egr1 mRNA induction in chondrogenesis is transient and precedes transcriptional upregulation of SOX9, a known key regulator of chondrogenesis. Acute loss of Egr1 prevents SOX9 induction, supporting a role for Egr1 in transcription of SOX9. In addition, loss of Egr1 leads to replication arrest in culture, which correlates with increased TRP53 and P $21^{\text {CIPWAF }}$ expression. In line with the above, loss of Egr1 has long-lasting effects on epigenomic reprogramming. Finally, we here describe a novel biological association between Egr1 and Polycomb Group function, providing at least a partial explanation for the observed defective differentiation in the Egr1-deficient model. 


\section{Materials and methods}

\section{Cell culture}

ATDC5 cells were cultured at $37^{\circ} \mathrm{C}, 5 \% \mathrm{CO} 2,100 \%$ humidity in DMEM/ $\mathrm{F}-12$ supplemented with $5 \%$ fetal calf serum (FCS), antibiotics (100 units $/ \mathrm{ml}$ penicillin and $100 \mu \mathrm{g} / \mathrm{ml}$ streptomycin), $200 \mathrm{mM}$ L-glutamine on tissue culture plates (Greiner Bio-One). For differentiation experiments cells were seeded at 6400 cells $/ \mathrm{cm}^{2}$ and were allowed to attach overnight. Growth medium was changed for differentiation medium, which includes ITS $\left(10 \mu \mathrm{g} / \mathrm{ml}\right.$ insulin, $10 \mu \mathrm{g} / \mathrm{ml}$ transferrin and $3 \times 10^{-8} \mathrm{M}$ sodium selenite). Differentiation medium was replaced every two days.

\section{Retroviral infections}

Ecotropic retroviral supernatants were produced by transfection of phoenix cells with calciumphosphate precipitation; 24-48 hours post-transfection, the supernatant was harvested, filtered and used for infection of ATDC5 cells in presence of $4 \mu \mathrm{g} / \mathrm{ml}$ polybrene. Cells were incubated for 12 hours and then allowed to recover for 24 hours on fresh medium before selection pressure was applied. Infected cells were selected with puromycin $(8 \mu \mathrm{g} / \mathrm{ml})$ for 72 hours, before experiments were initiated. Short-hairpin (sh)RNA target sequence for Egr1: 5'- ACAAAGTAACCTGTTTGGC3'. Short hairpin sequence targeting shGFP was used as reference sh sequence [44].

\section{Chrystal violet}

ATDC5 cells were plated in 12-multiwell plates (Greiner Bio-one). At each time point cells were washed twice with phosphate-buffered saline, and fixed for 10 minutes with $3.7 \%$ formaldehyde at room temperature. Next, cells were rinsed 5 times with demiwater. Cells were stained with $0.1 \% \mathrm{X}$ tal violet for 30 minutes or overnight, respectively, and washed 5 times with demiwater. X-tal was extracted with $10 \%$ acetic acid and absorbance was measured spectrophotometrically at $590 \mathrm{~nm}$ (Benchmark, Biorad).

\section{RNA isolation and cDNA synthesis}

Cells were suspended in Tri-reagent, and RNA was extracted according to the protocol of the manufacturer (Sigma). Quantity and purity of the RNA were determined by 260/280 nm absorbance measurements. cDNA was synthesized using iScript ${ }^{\mathrm{TM}} \mathrm{CDNA}$ synthesis kit (Biorad). Primers used are listed in supplemental Table 1.

\section{Western analysis}

Cells were lysed in RIPA buffer containing $5 \mathrm{mM}$ Benzamidine, $5 \mu \mathrm{g} / \mathrm{ml}$ Antipain, $5 \mu \mathrm{g} / \mathrm{ml}$ Leupeptin, $5 \mathrm{\mu g} / \mathrm{ml}$ Aprotinin, $1 \mathrm{mM}$ Sodium Vanadate, $10 \mathrm{mM}$ Sodium Fluoride, $10 \mathrm{mM}$ Pyrophosphate, $10 \mathrm{mM}$ ß-glycerophosphate, $0.5 \mathrm{mM}$ DTT and $1 \mathrm{mM}$ PMSF. Lysates were subjected to 3 freeze-thaw cycles in liquid nitrogen, and to 3 cycles of sonication. After centrifugation for 10 minutes at $13.2 \mathrm{krpm}$, protein concentration was determined using a BCA 


\section{Chapter 4}

protein assay kit (Pierce). Equal amounts of protein were boiled in Laemmli buffer and loaded on 9-15\% polyacrylamide gels. Proteins were transferred to polyvinylidene fluoride (PVDF) membranes. After blocking with $5 \%$ non-fat dry milk in PBS containing $0.1 \%$ Tween-20, membranes were incubated $\mathrm{o} / \mathrm{n}$ at $4^{\circ} \mathrm{C}$ with the following antibodies: anti $\beta$-actin Mab (C4, 691001, MP Biomedicals), anti Gapdh, anti Ezh2 Mab (BD43; coutesy D. Pasin Copenhagen, Denmark), anti Bmi1 Mab (F6; Courtesy M. van Lohuizen; Amsterdam, The Netherlands), anti Egr1 Poab sc-110 (Santa Cruz Biotechnology), anti Chk1-DCS-310 (Abcam Ab22610), anti p-Chk2 (Thr68) \#2661 (Cell signaling), anti $\gamma \mathrm{H} 2 \mathrm{~A} . X$ (Ser139) Poab \#2577 (Cell signaling), anti TRP53 Mab, anti geminin (FL-209) Poab (Santa Cruz Biotechnology), anti H3K4me3 Poab (ab8580) (Abcam), anti H3K27me3 Poab, \#07-449 (Upstate), anti Kap1 Poab A300-275A (Bethyl Laboratories, anti H3K9/14Ac Poab (\#06-599, Upstate Biotechnology). After extensive washing, membranes were incubated with corresponding horseradish peroxidase conjugated secondary antibodies for $1 \mathrm{~h}$ at room temperature. Signals were detected using enhanced chemoluminescence.

\section{Immunofluorescence}

Briefly, cells were washed and fixed for $10-15$ minutes in $100 \%$ methanol at $-20^{\circ} \mathrm{C}$ and stored at $4^{\circ} \mathrm{C}$ in $70 \%$ ethanol or used directly for immuno-histochemistry. Cells were permeabilized for $5-10$ minutes in $0.2 \&$ triton-X in PBS. To prevent epitope loss in combination with acid treatment for BrdU detection, primary antisera were incubated for 1.5 hours at $37^{\circ} \mathrm{C}, 4-5 \mathrm{x}$ washed in $0.02 \%$ triton-X/PBS and fixed in $2 \%$ formaldehyde/PBS for 10 minutes at room temperature. Cells were washed with PBS and incubated for 20 minutes in $2.0 \mathrm{~N} \mathrm{HCl}$ at $37^{\circ} \mathrm{C}$, followed by two rinses of 0.1 $\mathrm{M}$ sodium tetraborate solution, $\mathrm{pH} 8.5$ for in total 2 minutes. Cells were than incubated with the primary antisera against BrdU, washed at indicated before and incubated with secondary fluorescently labeled antibodies. All Antisera were diluted and incubated in $0.02 \%$ triton-X in PBS. Nuclei were counterstained with 4'-6-Diamidino-2-phenylindole (DAPI) and washed in $0.02 \%$ tritonX/PBS. The last wash step was in PBS, upon which cells were dehydrated: 1 minute in $70 \%$ ethanol, 2x 1 minute in 100\% ethanol and air-dried. Cells were mounted in Vectashield (Vector Laboratories, Inc. Burlingame, CA) and analyzed using a NIKON TE200 Eclipse fluorescence microscope and photographed using a NIKON DXM1200 digital camare in combination with NIS Elements 3.0 Imaging software. The following antibodied were used: anti YH2A.X Mab JBW301 (Upstate), anti BrdU (BD biosciences) and anti H3K9me3 \#07-442 (Upstate), anti H3K27me3 Poab, \#07-449 (Upstate). 


\section{Chromatin Immunoprecipitation}

ChIPs were performed and analyzed essentially as described previously ${ }^{51}$.

Briefly, U373 and HCT116 cells were fixed in 1\% formaldehyde. Cross-linking was allowed to proceed for $10 \mathrm{~min}$ at room temperature and stopped by addition of glycine at a final concentration of $0.125 \mathrm{M}$, followed by an additional incubation for $5 \mathrm{~min}$. Fixed cells were washed twice with PBS and harvested in SDS Buffer (50 mM Tris at pH 8.1, 0.5\% SDS, $100 \mathrm{mM} \mathrm{NaCl}, 5 \mathrm{mM}$ EDTA), supplemented with protease inhibitors (Aprotinin, Antipain and Leupeptin all at $5 \mu \mathrm{g} / \mathrm{mL}$ and $1 \mathrm{mM}$ PMSF). Cells were pelleted by centrifugation, and suspended in IP Buffer (100 mM Tris at pH $8.6,100 \mathrm{mM} \mathrm{NaCl}, 0.3 \% \mathrm{SDS}, 1.7 \%$ Triton $\mathrm{X}-100$, and $5 \mathrm{mM}$ EDTA), containing protease inhibitors. Cells were disrupted by sonication, yielding genomic DNA fragments with a bulk size of 200-500 bp. For each immunoprecipitation, $1 \mathrm{~mL}$ of lysate was precleared by addition of $35 \mu \mathrm{L}$ of blocked protein A beads (50\% slurry protein A-Sepharose, Amersham; $0.5 \mathrm{mg} / \mathrm{mL}$ fatty acid-free BSA, Sigma; and $0.2 \mathrm{mg} / \mathrm{mL}$ herring sperm DNA in TE), followed by clarification by centrifugation. $10 \mu \mathrm{l}$ aliquots of precleared suspension were reserved as input DNA and kept at $4^{\circ} \mathrm{C}$. Samples were immunoprecipitated overnight at $4^{\circ} \mathrm{C}$ with antibodies for either $\mathrm{HA}$ as a negative control (sc805; Santa Cruz), H3K27me3 (07-449; Upstate), anti H3K4me3 Poab (ab8580) (Abcam), anti Egr1 Poab sc-110 (Santa Cruz Biotechnology). Immune complexes were recovered by adding $40 \mu \mathrm{L}$ of blocked protein $A$ beads and incubated for $4 \mathrm{~h}$ at $4^{\circ} \mathrm{C}$. Beads were washed three times in $1 \mathrm{~mL}$ of Mixed Micelle Buffer $(20 \mathrm{mM}$ Tris at $\mathrm{pH} 8.1,150 \mathrm{mM} \mathrm{NaCl}, 5 \mathrm{mM}$ EDTA, $5 \% \mathrm{w} / \mathrm{v}$ sucrose, $1 \%$ Triton $\mathrm{X}-100$, and $0.2 \%$ SDS), twice in $1 \mathrm{~mL}$ of Buffer 500 (50 mM HEPES at pH 7.5, 0.1\% w/v deoxycholic acid, $1 \%$ Triton X-100, and $1 \mathrm{mM}$ EDTA), twice in $1 \mathrm{~mL}$ of LiCl Detergent Wash Buffer (10 mM Tris at $\mathrm{pH} 8.0,0.5 \%$ deoxycholic acid, 0.5\% NP-40, $250 \mathrm{mM} \mathrm{LiCl}$, and $1 \mathrm{mM}$ EDTA), and once in $1 \mathrm{~mL}$ of TE. Immuno-complexes were eluted from beads in $250 \mu \mathrm{L}$ elution buffer (1\% SDS; and $0.1 \mathrm{M} \mathrm{NaHCO} 3$ ) for $2 \mathrm{~h}$ at $65^{\circ} \mathrm{C}$ with continuous shaking at $1000 \mathrm{rpm}$, and after centrifugation supernatants were collected. $250 \mu \mathrm{L}$ elution buffer was added to input DNA samples and these were processed in parallel with eluted samples. Crosslinks were reversed overnight at $65^{\circ} \mathrm{C}$ followed by a $2 \mathrm{~h}$ digestion with RNAse $A$ at $37^{\circ} \mathrm{C}$ and $2 \mathrm{~h}$ proteinase $\mathrm{K}(0.2 \mu \mathrm{g} / \mu \mathrm{L})$ at $55^{\circ} \mathrm{C}$. DNA fragments were recovered using QIAquick PCR purification columns, according to manufacturers' instructions. Samples were eluted in $75 \mu \mathrm{L}$ EB buffer and then further $1 / 5$ diluted in TE buffer. The immunoprecipitated DNA was quantified by real-time qPCR. Primers used are listed in supplemental Table 1.

\section{Affymetrix gene arrays}

Affymetrix NuGO mouse arrays ( $24 k)$ were run on RNA from ATDC5 cultures sampled over time post induction of differentiation (pid). For each time point 3 samples were taken from both a wildtype (WT) and a Egr1 knock-down (KD) culture. After scanning, data preprocessing and analysis were done using R2.7.1 with the use of Bioconductor libraries ${ }^{80,81}$. Data were ${ }^{2} \log$ transformed and normalized using gcRMA ${ }^{82}$. 
Chapter 4

\section{Results}

\section{Egr1 is rapidly induced in differentiation}

To identify early responses to differentiation stimuli, ATDC5 cells were stimulated to undergo chondrogenesis. An expression-array experiment was designed to assay immediate early, early and late changes in gene expression profiles, as a function of time $(0,2,4,8,16,24$ and $72 \mathrm{hrs})$. Expression analysis revealed a rapid upregulation of a number of immediate early growth response genes (IEGs): expression of IEGs belonging to the FOS, JUN and EGR1 subfamilies is significantly enhanced within 2 hours upon induction of chondrogenesis in murine mesenchymal progenitor ATDC5 cultures (Suppl. Figure 1A). Also c-MYC is induced, albeit at more moderate levels (2x; FS, GvdA, JWV; unpublished data).

A

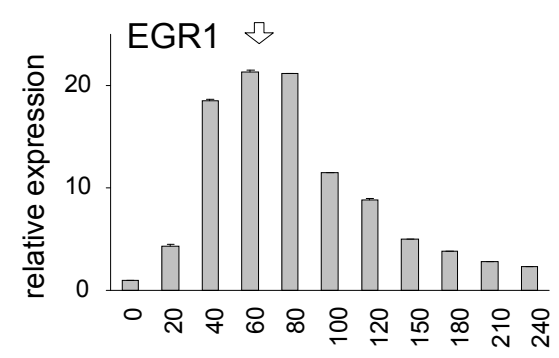

time pid (minutes)

C

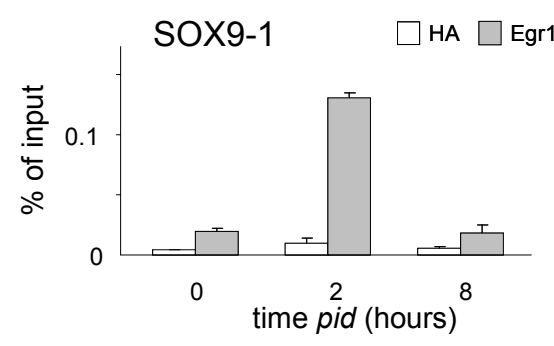

E

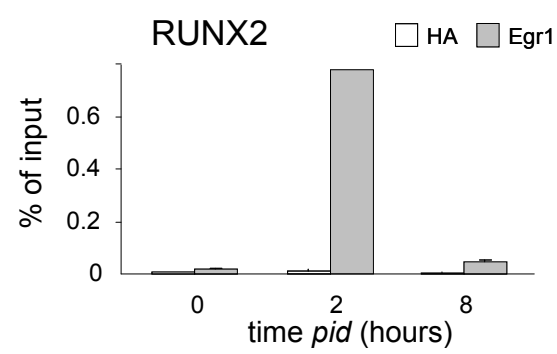

G

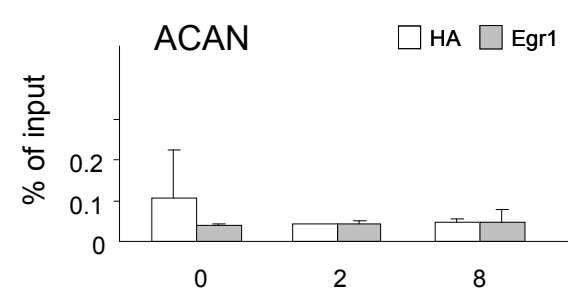

B

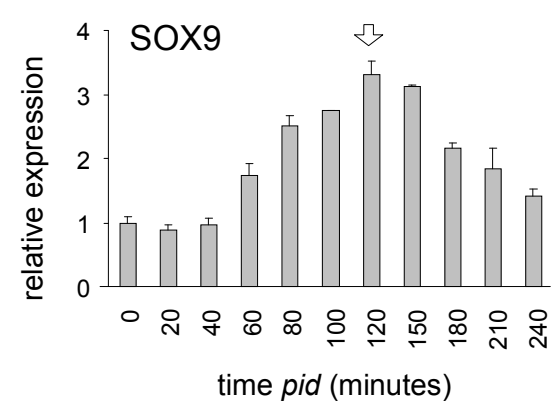

D $\square$ HA $\square$ H3K27me3 $\square$ H3К4mе3

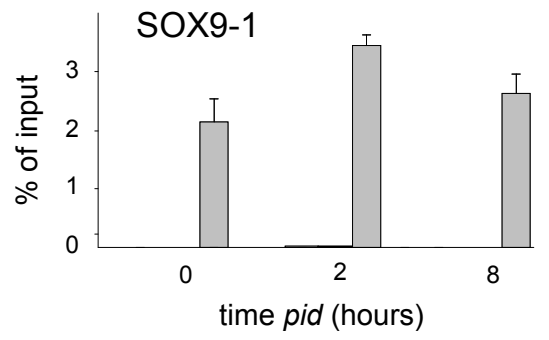

$\mathbf{F}$

$\square$ HA $\square$ H3K27me3 $\square$ H3K4me3

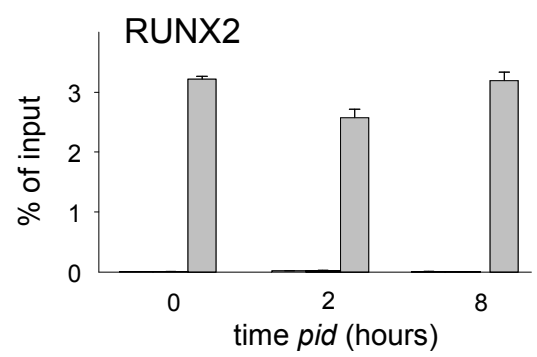

H $\square$ HA $\square$ HзK27me3 $\square$ HзК4mе3

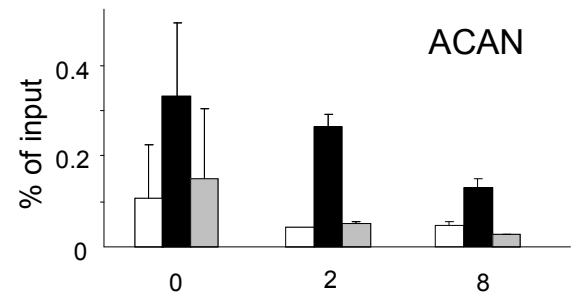


Figure 1. Egr1 is rapidly induced in chondrogenic differentiation and binds to chondrogenic promoters.

(A) qRT rtPCR analysis of Egr1 expression up to 4 hours pid with 20 or 30 minute intervals. (B) qRT rtPCR analysis of Sox9 expression up to 4 hours pid with 20 or 30 minute intervals. (C, E, G) ChIP using anti-Egr1 antibodies and n; 0,2 and 8 hours pid immunoprecipitated DNA was analyzed by qRT rtPCR for the respective promoters. (D,F,H) ChIP using anti-H3K27me3 and anti-H3K4me3 antibodies and n; 0, 2 and 8 hours pid immunoprecipitated DNA was analyzed by qRT rtPCR for the respective promoters.

Expression of most IEGs rapidly declines over the next 2 hours. To investigate a role for IEGs in chondrogenesis we focused on EGRs; EGR1 and EGR3 are both massively induced upon adding ITS: EGR1 expression is induced up to $200 \mathrm{x}$ at the mRNA and returns to baseline levels within 4 hours pid (post-induction of differentiation). An independent real time expression analysis using a time resolution of 20 minute intervals essentially confirmed the microarray findings: EGR1 mRNA increases within 20 minutes into differentiation, peaks at 1-2 hours and falls off to pre-induction levels within 2-3 hours (Figure 1A). EGR1 expression increases again later during chondrogenesis around 6 days (Suppl Figure 1B). Protein expression parallels the observed changes in EGR1 mRNA levels nicely: whereas absent at $\mathrm{t}=0$, Egr1 protein is first detected at 1 hour pid, peaks at 2 hours and rapidly declines thereafter (Figure 2A).

\section{Egr1 initiates chondrogenesis through SOX9 and RUNX2 expression}

This early IEG-induction profile suggested a role for EGR1 in activating downstream differentiation programs. To chart potential target genes for Egr1 and to build a chondrogenic gene-interaction network, we combined published data, with a mouse genome-wide screen for Egr1 binding sites and software tools to predict promoter binding sites for numerous transcription factors. Multiple consensus binding sequences for Egr familiy members exist ${ }^{27-29}$ we confined our genomic scan to GCGG/TGGGCG motifs and its reverse complementary sequence, both in sense and antisense orientation. Genes containing predicted binding sites were mapped using pathway analysis tools (Pathvisio with Wikipathways content; Suppl Figure 2). Several known key-regulators of chondrogenesis, such as SOX9 and RUNX2 ${ }^{30-33}$, contain predicted Egr1 binding sites. At the mRNA level, SOX9 shows a transient, approximately 1 hour out-of-phase induction in response to differentiation medium as compared to EGR1 (Figure 1B). In silico SOX9 promoter analysis using GENOMATIX software revealed several putative binding sites for Egr family members a number of which correspond to potential Egr1 consensus binding sites (Suppl Figure 3). To confirm the in silico mapping, we performed chromatin immunoprecipitation (ChIP) with anti-Egr1 antibodies on ATDC5 cells differentiated for 0,2 and 8 hours. Two independent primer sets were designed to detect co-precipitated SOX9 promoter sequences using real time PCR. In good agreement with the expression kinetics of EGR1 upon induction of differentiation, both SOX9 primer sets show substantial enrichment of Egr1 within the vicinity of the Egr1 binding sites at 2 hours pid (10-15 fold; Figure 1C; Suppl Figure 4A). Likewise, the RUNX2 promoter is enriched 67-fold for Egr1 at 2 hrs (Figure 1E). Both promoters carry histone $\mathrm{H} 3 \mathrm{~K} 4 \mathrm{me} 3$, an epigenetic status permissive for transcriptional activity (Figure 1D, F; Suppl Figure 4B). Consistent with a sharp drop in protein 


\section{Chapter 4}

levels (Figure 2A), 8 hours into differentiation, Egr1 occupation at these promoters was restored to levels found in undifferentiated cells. Conversely, the Aggrecan (ACAN) promoter, appears not enriched for Egr1 at 2 hours pid, and, in contrast to the SOX9 and RUNX2 promoters, ACAN is marked for repression with H3K27me3 (Figure 1G, H). Both findings are consistent with a significantly later transcriptional activation of ACAN in chondrogenesis as opposed to SOX 9 and RUNX2 at approximately 10 days (Suppl Figure $4 \mathrm{C}-\mathrm{H}$ ). To firmly establish a role for Egr1 in chondrogenesis, we studied chondrogenic marker gene expression in the context of RNAinterference mediated knock-down (KD) of EGR1. A shRNA vector targeting murine EGR1 mRNA (shEGR1) was designed based on criteria published before ${ }^{34}$. Expression analysis at both mRNA level and protein level shows that the retroviral shEGR1 vector efficiently targets EGR1 expression (Figure 2A,B). Both SOX9 and RUNX2 display a multi-phasic expression profile throughout differentiation (Suppl Figure 4C-F). Relevantly, loss of EGR1 function (EGR1-LOF) blunts early SOX9 and RUNX2 expression (Figure 2C; Suppl Figure 4D). Egr1 enrichment in the SOX6 promoter is only marginally changed and EGR1-LOF appears to have very little effect on mRNA expression (Suppl Figure 5A,D).

A

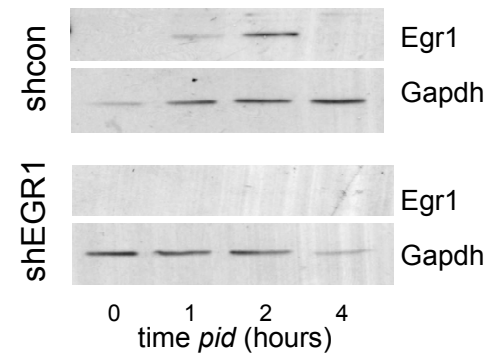

C

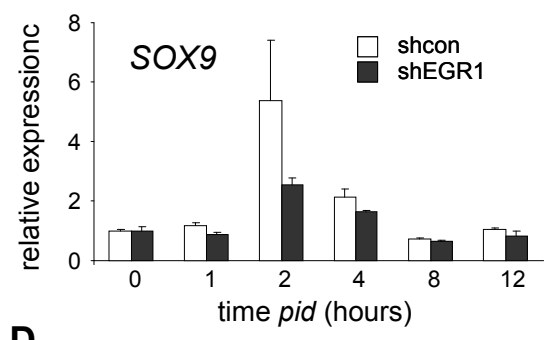

D

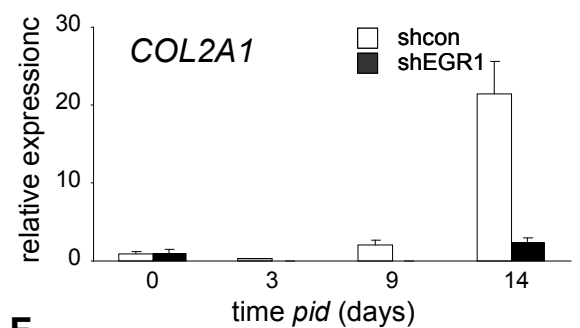

$\mathbf{F}$

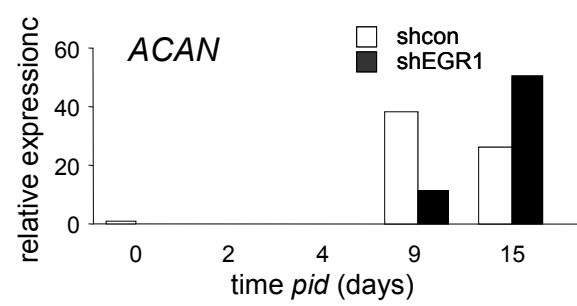

B

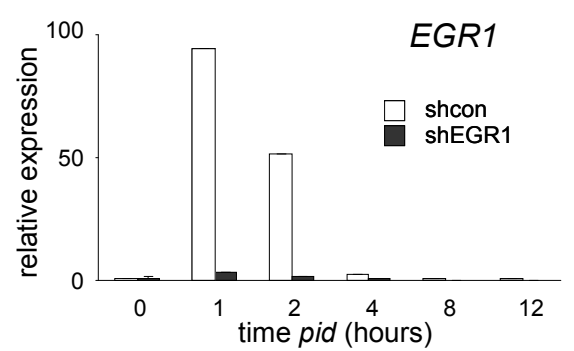

E
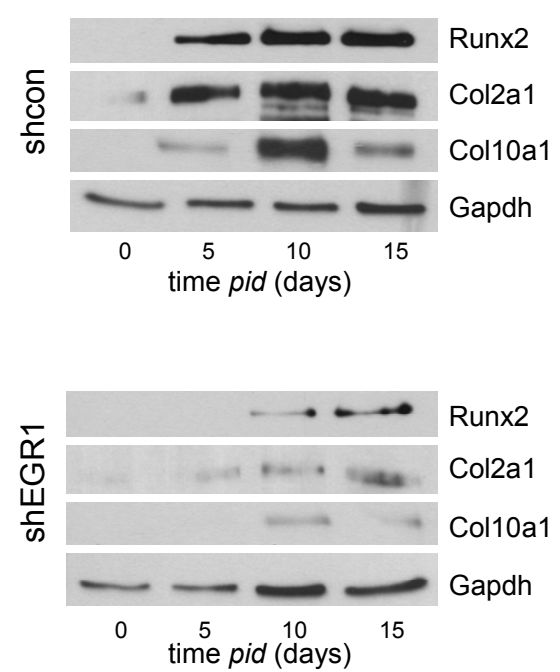
Figure 2. Egr1 is required for induction of chondrogenic differentiation.

(A) Western blot analysis of Egr1 expression in ATDC5 cells stably expressing shControl or shEgr1 at $0,1,2$ and 4 hours pid. GAPDH is used as loading control (B) qRT rtPCR analysis of Egr1 expression in ATDC5 cells stably expressing shControl or shEgr1 up to 4 hours pid. (C) qRT rtPCR analysis of Sox9 expression in ATDC5 cells stably expressing shControl or shEgr1 up to 12 hours pid. (D,F) qRT rtPCR analysis of collagen 2a1 and agrrecan expression in ATDC5 cells stably expressing shControl or shEgr1 up to 15 days pid. (E) Western blot analysis of chondrogenic marker expression in ATDC5 cells stably expressing shControl or shEgr1 up to 15 days pid.

This suggests that SOX6 and SOX4 loci may not be prime Egr1 targets for transcriptional activation early in chondrogenesis; indeed both SOX6 and SOX4 are not expressed until later in chondrogenesis (Suppl Figure 5C). Expression of genes encoding the ECM proteins Collagen type II (Col2A1) and Acan is activated 7-10 days pid in reference cultures; Egr1-KD significantly reduces COL2A1 and ACAN expression at the mRNA and protein level (Figure 2D,E; Suppl Figure 4C). RUNX2-induced Collagen type X (COL10A1) expression coincides with hypertrophic differentiation ${ }^{35}$; Col10A1 levels are severely reduced in Egr1-KD cultures (Figure 2E). Thus, activation of Egr1 early in chondrogenesis directly activates transcription of key regulators of downstream differentiation pathways.

\section{Loss of Egr1 elicits replication stress induced senescence-like cell cycle arrest.}

An important feature of early chondrogenesis is the capacity of chondrocytes to rapidly proliferate. We studied ATDC5 differentiation induced hyperproliferation as a function of EGR1 expression. Proliferation rate of control cells is approximately 3 fold increased from 1 day pid onward as compared to cells under normal, non-differentiating culture conditions (Figure 3A). Differentiating cells typically overgrow each other and form nodules, i.e focal points of chondrogenesis (Suppl Figure 6). The proliferative burst coincides with a second period of EGR1 upregulation (Suppl Figure 1B), indicating a potential requirement for EGR1 during enhanced cell division. Remarkably, EGR1-LOF completely abrogates the ability of ATDC5 cells to hyperproliferate and cells cease proliferation when they reach confluency (Figure 3B; Suppl Figure 6). The proliferative block was independently confirmed using incorporation of $\mathrm{BrdU}$ as a read-out (Figure $3 \mathrm{C}$ ). The hyperproliferative block is accompanied by dramatic morphological changes: EGR1-LOF cells display the 'large flat cell' phenotype typically associated with cellular senescence (Figure 3D). Consistent with this, ATDC5 EGR1-LOF cultures show polyploidy and reveal transcriptional induction of numerous genes implicated in cellular senescence, DNA damage and stress signaling (Suppl Figure 7). 
A

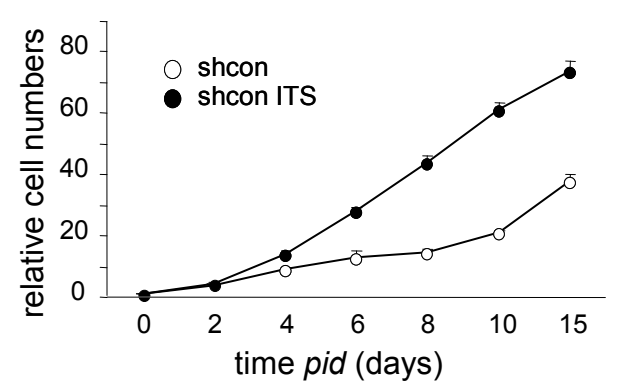

C

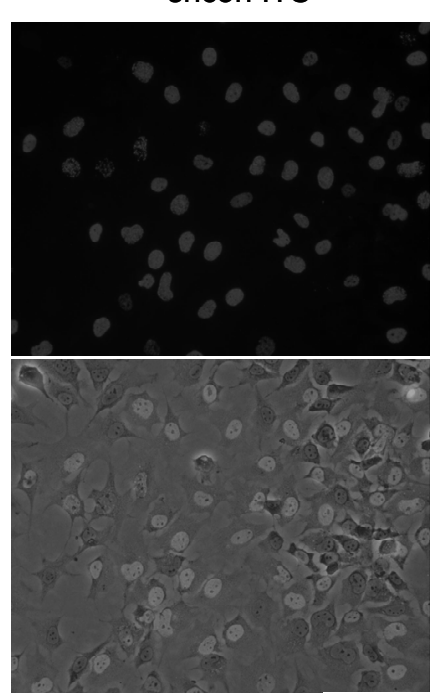

$\mathrm{t}=2$ pid (days)

D

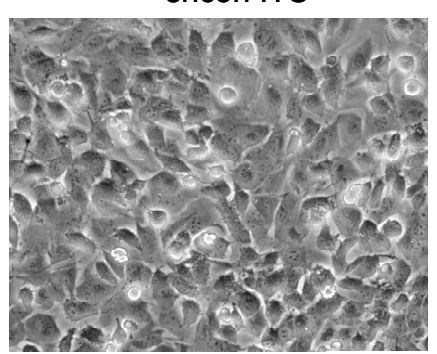

B

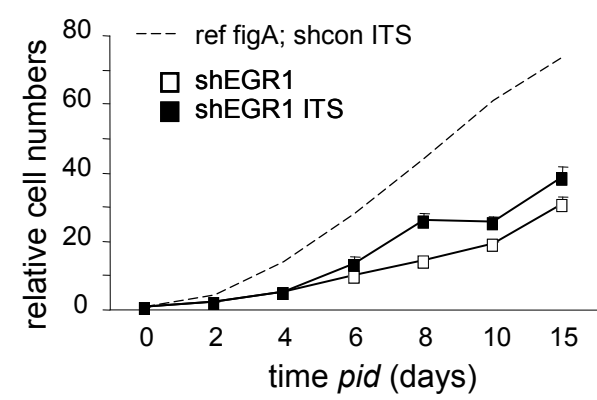

shEGR1 ITS

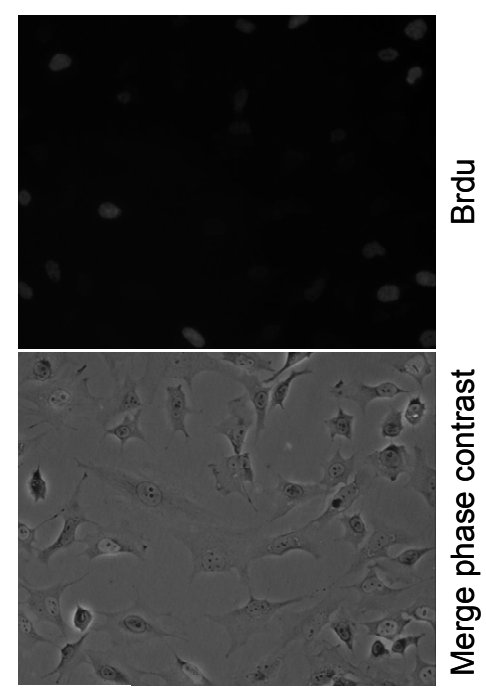

shEGR1 ITS

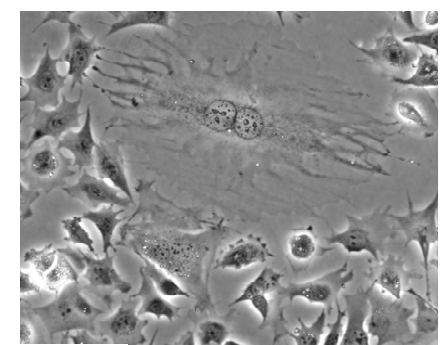

$\mathrm{t}=4$ pid (days)

Figure 3. Loss of Egr1 elicits replication stress induced senescence-like cell cycle arrest.

(A) ATDC5 cells hyperproliferate upon induction of chondrogenic differentiation. Proliferation determined by Crystal violet incorporation with subsequent extraction and spectrophotometric analysis. (B) ATDC5 cells stably expressing shEgr1 fail to undergo differentiation induced hyperproliferation, measured by Crystal violet incorporation. (C) ATDC5 cells stably expressing shEgr1 or shControl were differentiated for 2 days, then BrdU was administered to the cells for 45 minutes. BrdU was visualized with anti-BrdU antibodies. (D) Morphological analysis of ATDC5 cells stably expressing shEgr1 or shControl at 4 days pid.

Activation of a DNA damage response (DDR) was confirmed by immunoblotting of phosphorylated H2A.X ( $\gamma \mathrm{H} 2 \mathrm{~A} . \mathrm{X})$ and Chk2 in chondrogenic cultures (Figure 4A-B). In control cells Chk2 is phosphorylated at a basal level and slightly reduced during hyperproliferation, differentiation in the absence of Egr1 induces a substantial increase in pChk2 at day 3 (Figure 4A). Upregulation of 
several cell stress markers suggests that the Atm/Atr DDR (pChk2, $\gamma \mathrm{H} 2 \mathrm{~A} . \mathrm{X}$; Chk1) pathway and cell cycle arrest (Trp53, Gmnn) are activated. Consistent with this, immunohistochemical staining for $\gamma \mathrm{H} 2 \mathrm{~A}$.X confirms DNA damage in Egr1-KD cells under differentiating conditions (Figure 4C). To map processes regulated by Egr1, the genes identified in a genome-wide blast for Egr1 binding sites were analyzed using GenMAPP; this yielded a number of overrepresented processes known to be involved in chondrogenesis and differentiation in general (table 1).

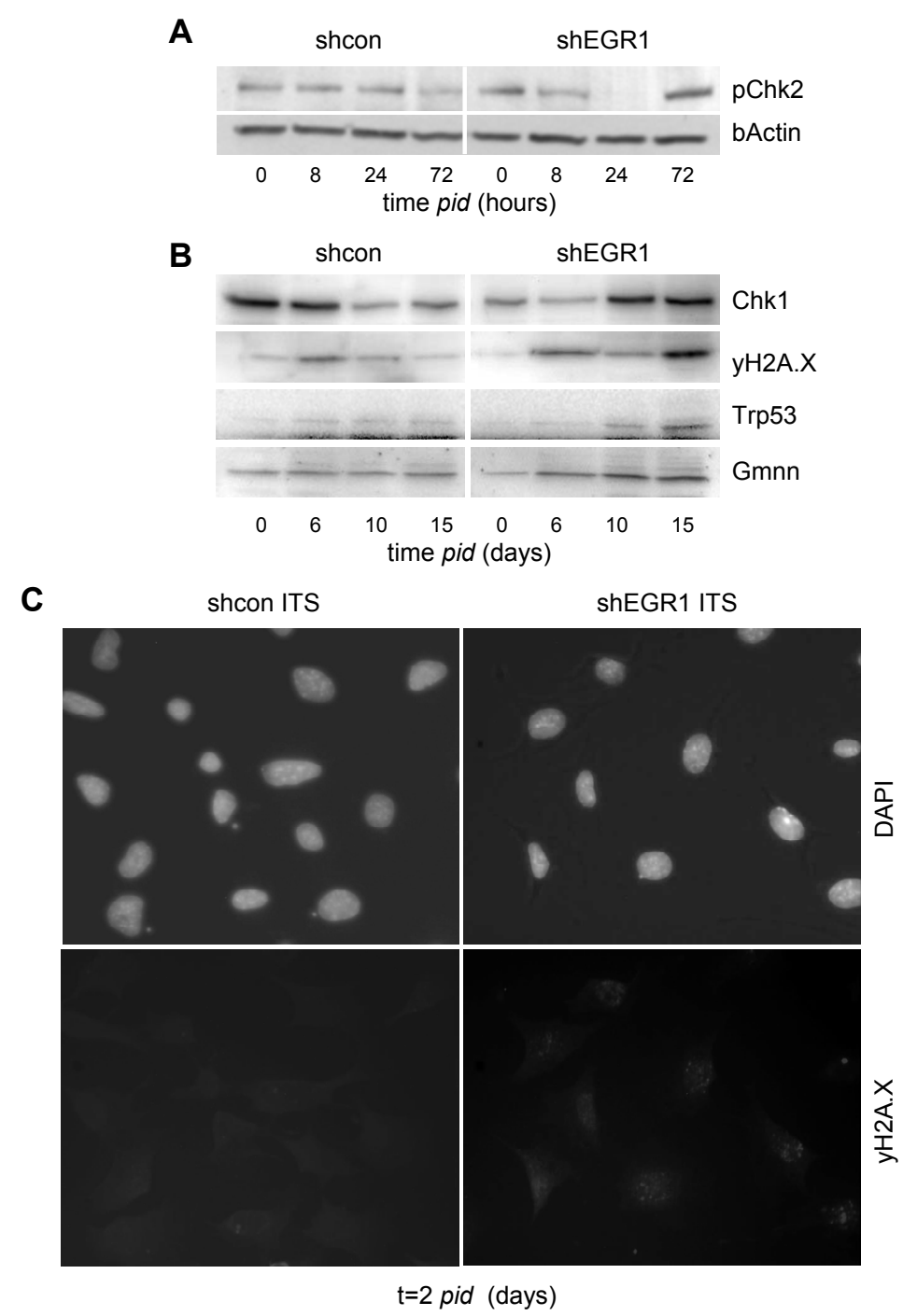

Figure 4. Egr1-LOF elicits DNA damage in transit amplifying chondrocytes

A, B) Western blot analysis of proteins related to DNA damage responses in ATDC5 cells stably expressing shControl or shEgr1. bActin is used as loading control. (C) Protein expression of $\mathrm{yH} 2 \mathrm{Ax}$ in ATDC5 shControl and shEgr1, at 2 days pid. Counterstaining by DAPI.

In addition, we identified cytokine signalling pathways as potential targets of Egr1. Relevantly, interleukins, like IL6, are known to play an important role in establishment of senescence ${ }^{36,37}$. In good agreement with this, IL6 is significantly induced upon differentiation in the absence of Egr1 (Suppl Figure 7). Taken together, this data clearly identifies Egr1 as an essential regulator protein for hyperproliferation in the context of chondrogenesis and shows loss of Egr1 results in cellular senescence-like response. 


\section{Chapter 4}

\section{Egr1 controls epigenomic remodeling}

As Egr1 controls both transcriptional reprogramming and hyperproliferation, we studied global epigenomic changes throughout chondrogenesis as a function of Egr1. Histone acetylation (H3K9/K14ac and H4K12ac), trimethylation at lysine 27 (H3K27me3), lysine 4 (H3K4me3) and lysine 9 (H3K9me3) all undergo dynamic changes throughout chondrogenesis: all trimethyl marks peak during hyperproliferation and gradually increase toward hypertrophy (Figure 5A). In contrast, histone acetylation is high in shcon chromatin at the initial stages of chondrogenesis, and gradually declines over time (Figure 5A). Remarkably, all epigenomic changes were substantially perturbed in Egr1-KD cultures. Consistent with a role in recruiting HAT (histone acetyl transferase) activity, EGR1-LOF cells display a striking inability to induce and/or maintain acetylation levels throughout hyperproliferation (Figure 5B). In contrast, trimethylation levels at histone H3K4, H3K9 and H3K27 appear initially low and substantial elevate at later time points in the absence of Egr1 (Figure 5B, C). We next investigated the effect of Egr1-KD on a number of H3K27me3 and K9me3-associated proteins. Polycomb Group proteins are known to affect skeletogenesis in vivo. The Polycomb PRC2 and PRC1 complex are both functionally connected to the H3K27me3 mark. Egr1-KD cultures fail to properly induce BMI1 both at the mRNA and protein level, suggesting a direct effect of EGR1 on BMI1 expression (Figure 5D; Suppl Figure 8A). Also Ezh2 expression is deregulated in Egr1-KD cultures: under normal conditions Ezh2 protein levels slightly increases in response to early signaling in chondrogenesis; this induction fails in Egr1-KD cultures (Figure 5E; Suppl Figure 8A). Conversely, Ezh2 is substantially induced in Egr1-KD cells at late time points in differentiation (Figure 5E). The enhanced Ezh2 expression correlates well with the increase in H3K27me3 (Figure 5C,G).

The presence of multiple predicted EGR-family member consensus binding sites in the Bmi1 promoter, and a more than 50 fold increased Egr1 presence at the Bmi1 promoter (Suppl. Figure $8 \mathrm{C}, \mathrm{D})$ are consistent with direct control of Bmi1 expression by Egr1. The Ezh2 promoter is substantially less enriched for Egr1 ( \pm 4 fold) and Ezh2 mRNA levels are not consistently suppressed in the absence of Egr1 arguing for a possibly secondary effect of EGR1-LOF on expression of Ezh2. (Suppl Figure 8B,E). A substantial increase of KAP1/TIF1B expression, a factor associated with $\mathrm{HP} 1 / \mathrm{H} 3 \mathrm{~K} 9 \mathrm{me} 3$, correlated well with the observed increase in $\mathrm{H} 3 \mathrm{~K} 9 \mathrm{me} 3$ levels at late time points in Egr1 LOF cultures (Figure 4C,F,G). In summary, the above data demonstrate that Egr1 is crucial for initiation of an epigenome wide reprogramming during chondrogenesis and that differentiation in the absence of Egr1 results in abnormal epigenetic responses. 
A

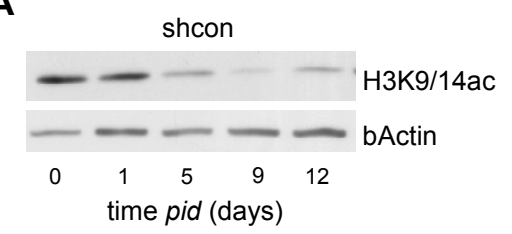

C

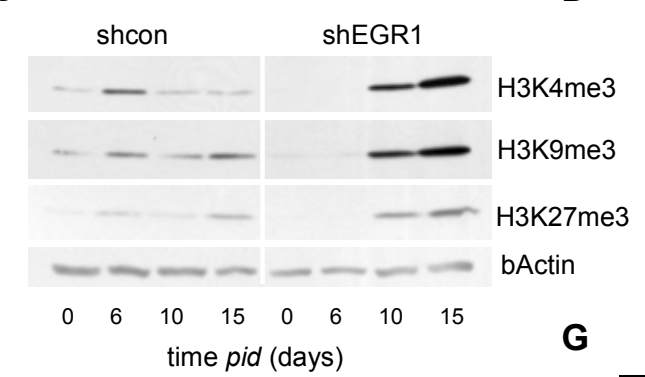

E

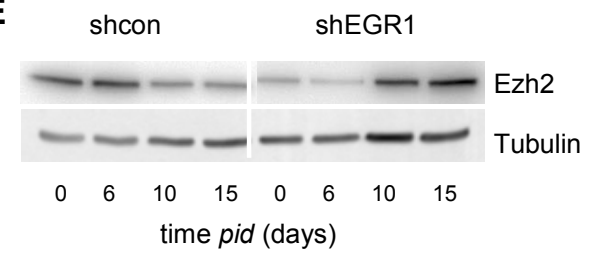

$\mathbf{F}$

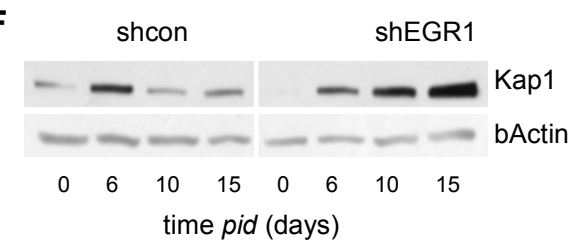

B

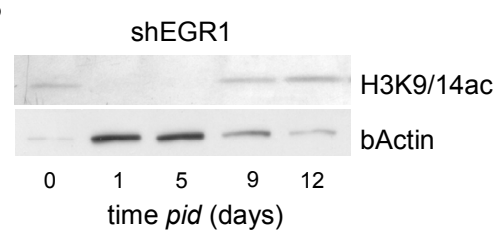

D

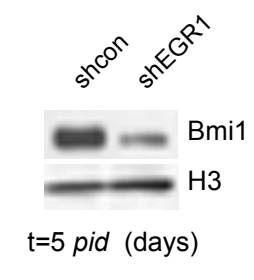

shcon shEGR1
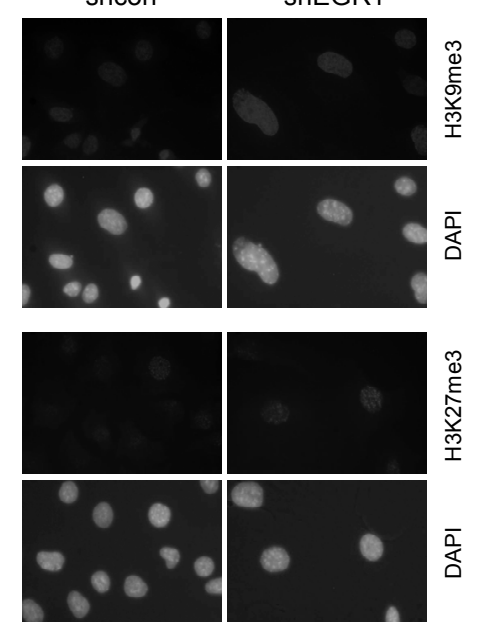

Figure 5. Loss of Egr1 affects histone modifications and histone modifiers.

$(A, B, C)$ Westernblot analysis of histone modifications in ATDC5 shControl and shEgr1 throughout chondrogenesis. (D,E,F) Westernblot analysis of PcG proteins and Kap1, a novel PcG interacting protein, in ATDC5 cells stably expressing shControl or shEgr1. (G) Immunocytochemical analysis histone modifications in ATDC5 cells stably expressing shControl or shEgr1.

\section{Egr1-promoter binding is not sufficient for transcriptional activation}

The genome-wide abundance of Egr1-family consensus binding sites and of Egr1 in particular, combined with the dramatic effects of Egr1-LOF on global epigenomic reprogramming during chondrogenesis suggested that Egr1 may not enhance transcriptional activity by itself upon DNA binding. The SOX9 promoter is predicted to be in a relatively 'open' chromatin configuration at $\mathrm{t}=0$, based on the presence of transcriptional activity-associated H3K4me3 marks and the absence of repressive $\mathrm{H} 3 \mathrm{~K} 27 \mathrm{me} 3$ marks. This chromatin state is maintained early in chondrogenesis (Figure 1D). The same holds true for RUNX2, which, as SOX9, is free of repressive H3K27me3 marks (Figure 1F), and for PRC gene promoters of BMI1 and EZH2 (Suppl Figure 8F, G). All 'open' chromatin promoters, i.e. only marked H3K4me3, appear enriched for Egr1 at $2 \mathrm{hrs}$ of induction of 


\section{Chapter 4}

differentiation. Yet, not all these "open" genes neither follow the induction profile typical of e.g. SOX9, nor lose induction of gene activity in the absence of Egr1 as SOX9 does. In contrast, gene promoters of $A C A N$ and SOX6, appear initially silenced by PRC1 (Figure 1H, Suppl Figure 5B): the ACAN promoter carries $\mathrm{H} 3 \mathrm{~K} 27 \mathrm{me} 3$ marks, consistent with ACAN being a PRC1 target in cells of mesenchymal origin ${ }^{11}$. Indeed, a comparison of chondrogenic promoters with putative Egr1binding sites and reported Polycomb-associated gene promoters yielded a number of potential common target genes for both Egr1 and Polycomb (Suppl Figure 2). SOX6, a presumed Sox9 target, gradually loses its PRC-associated repressive mark over the initial 8 hrs in differentiation. Regardless of these initial epigenetic conversions, however, neither ACAN nor SOX6 are transcriptionally activated until late in chondrogenesis. Neither gene is substantially enriched for Egr1, despite the presence of predicted Egr1 binding sites in their promoters (Suppl Figure 3) suggesting that a repressive chromatin confirmation prevents Egr1 access.

To independently test the hypothesis that Egr1 binding does not induce gene activity per se, we analyzed HOX cluster gene expression in the context of Egr1 presence. HOX genes are key developmental regulators of anterio-posterior (AP) patterning and skeletal development ${ }^{38,39}$. HOX genes are functionally grouped in 4 multi-gene clusters in mammals; expression within these clusters is subject to co-linear regulation, hence expression boundaries within a cluster are sharply defined in a cell-context specific manner ${ }^{40,41}$. HOX clusters carry predicted Egr1 binding sites (Suppl Figure 9B).

To examine induction of HOX gene activity in relation to Egr1 enrichment, HOXA cluster genes (A1, A4, A6, A7, A9 and A11) were subjected to ChIP analysis for Egr1, H3K27me3 and H3K4me3 during early chondrogenic differentiation. Relevantly, a gradient of epigenetic marking over the HoxA cluster is readily visible: HOXA1 and HOXA4 promoters are up to 400 -fold enriched for H3K4me3, whereas HOXA6 shows a lower, approximately 20 -fold enrichment compared to the HA-control (Figure 6A). None of these HOXA genes are enriched for H3K27me3. The HOXA7 promoter appears only moderately enriched for both epigenetic modifications, whereas HOXA9 and HOXA11 lack H3K4me3, they display enrichment for the repressive H3K27me3 mark (Figure $6 \mathrm{~A})$. These findings predict an expression boundary within the HOXA cluster at HOXA6/A7 in nondifferentiated ATDC5 cells. Strikingly, Egr1 is only enriched at epigenetically accessible HOXA promoters, as defined by $\mathrm{H} 3 \mathrm{~K} 4 \mathrm{me} 3-$ marking; none of the H3K27me3 promoters are enriched (Figure 6B). Despite the increased Egr1 occupation at 2 hours pid, Egr1-binding does not induce transcriptional regulation of any gene within the HOXA cluster, irrespective of epigenetic status (Figure 6C). Consistent with this, none of the Hox gene clusters respond to EGR1-LOF (Suppl Figure 9). Taken together the above data shows that Egr1 binding of consensus sequence motifs is not sufficient for activation of transcription. 
A
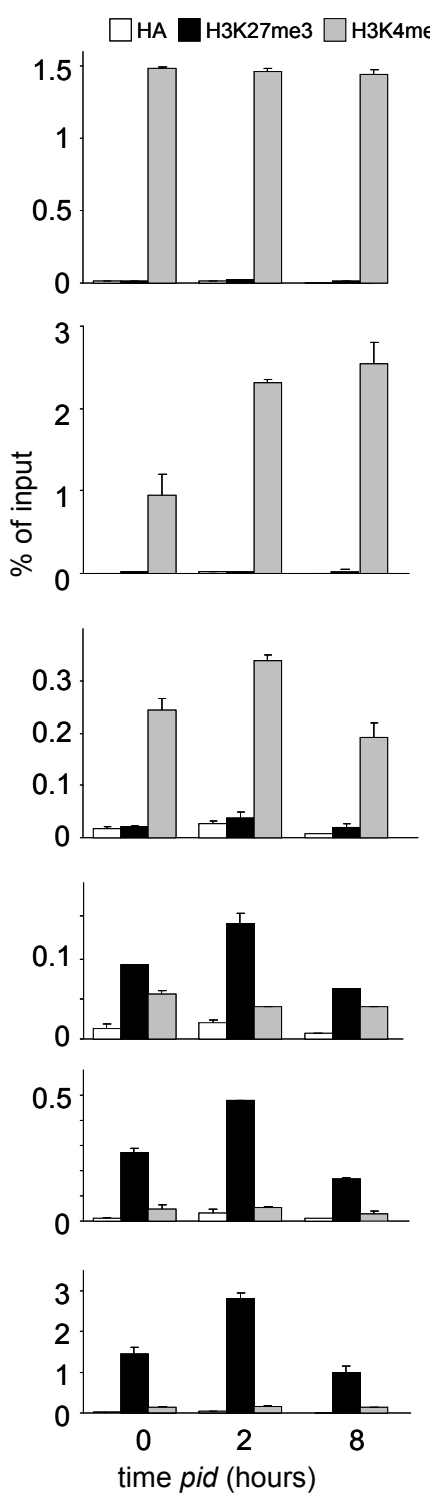

B
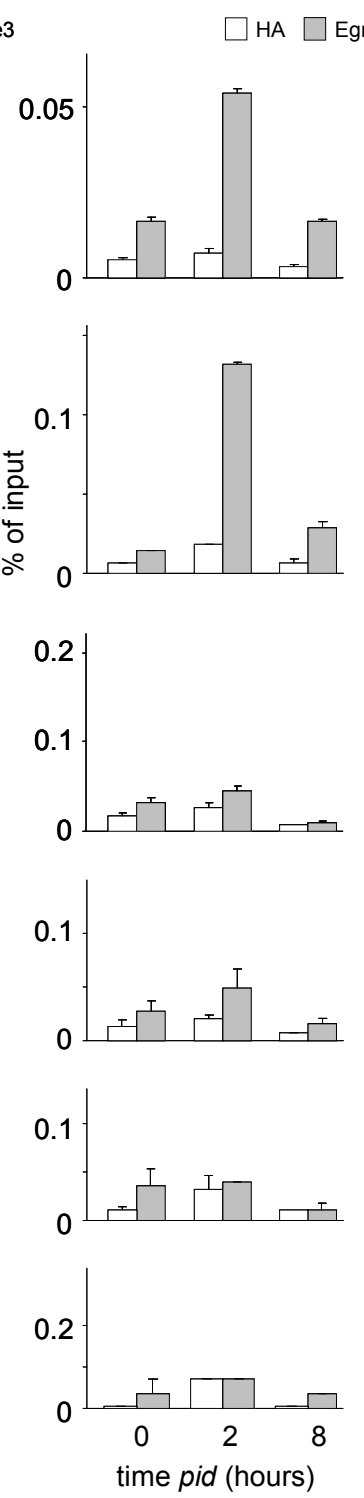

C
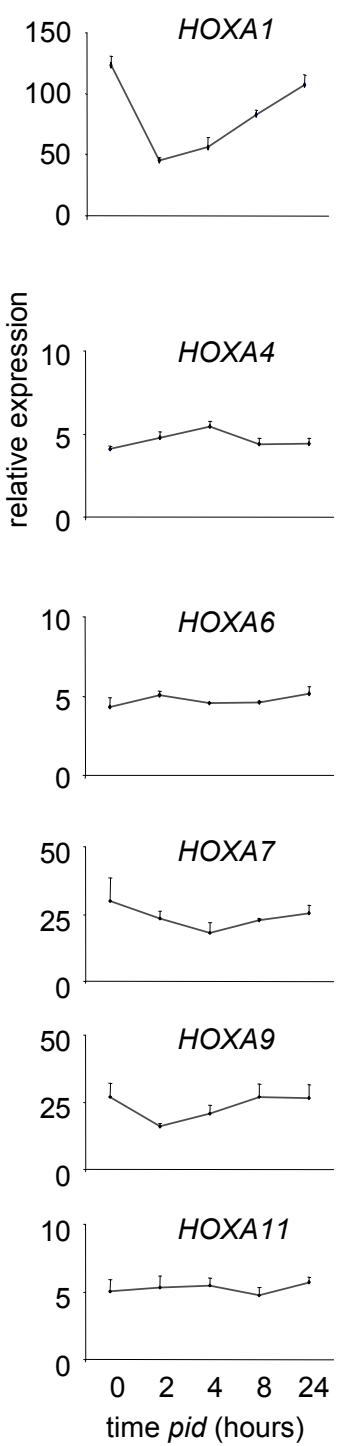

Figure 6. Egr1-promoter binding is not sufficient for transcriptional activation. (A,B) ChIP analysis for enrichment of H3K27me3, H3K4me3 and Egr1 on promoters of the HoxA cluster. (C) Relative mRNA expression of the HoxA cluster in ATDC5 cells differentiated for up to 24 hours.

\section{Discussion}

We here report for the first time that many IEGs are rapidly induced in the context of chondrogenesis: Egr1 is highly induced and controls relevant chondrogenic pathways: RNA interference-mediated knock-down of Egr1 affects early global chromatin acetylation and severely impedes chondrogenesis: both expression of key regulator genes of chondrogenesis, like SOX9 and RUNX2, as well as transit amplification are blocked. The block in rapid progenitor expansion correlates with DNA-damage induced signaling and display characteristics of a senescence-like response. Our analysis also shows that Egr1 promoter binding does not activate transcription per se, and suggests a more general role for Egr1 in epigenomic reprogramming during differentiation. 


\section{Chapter 4}

The observation that Egr1-KD specifically blocks rapid differentiation-induced progenitor expansion strongly suggests that replication stress is involved in the proliferative block. The conditions at onset of differentiation show remarkable resemblance to oncogenic stress and several lines of evidence suggest parallels with oncogene induced senescence (OIS). Firstly, ATDC5 cells undergo a transit proliferative amplification, a situation identical to the early increase in proliferation induced by oncogenes ${ }^{42-44}$. Secondly Egr1-KD cells accumulate DNA damage and activate a DDR, as evidenced by phosphorylation of H2AX, Chk2 and Kap1, and upregulation of multiple DDR genes. Thirdly, in the context OIS, cell cycle exit and irreversible senescence are dependent on activation of a DDR ${ }^{43}$. We show here that DDR in Egr1-KD cells coincides with a strong replicative block and a senescence-like phenotype: cells display distinctive features associated with senescence: large flat cell morphology, polyploidy, expression of numerous senescenceassociated marker genes. Combined this data strongly argues that Egr1 facilitates proliferative expansion in transit amplifying chondrogenic progenitors.

The early abnormal global acetylation in the absence of Egr1 is in good agreement with Egr1's ability to recruit HAT activity ${ }^{45}$. C-Myc, one of the first IEGs identified, was recently found to augment global acetylation through both transcriptional regulation and recruitment of GCN5 ${ }^{46}$. Early acetylation may be required for making rapid proliferation possible. It is conceivable that global acetylation in response to DNA binding of IEG-encoded proteins may help define regions within a network of increased DNA-templated activity, and support differentiation-specific changes in transcription and proliferation.

It is currently only possible to speculate about the biological relevance of the co-regulation of seemingly conflicting trimethyl levels on H3K4, H3K9 and H3K27 under normal and stressed conditions. It seems unlikely, given their stability across cell generations, that these changes in global trimethylation reflect a cell cycle dependent enrichment. Besides with transcriptional activity, each mark is associated with distinctive other processes. H3K9me3 is a hallmark of heterochromatin. H3K9me3 and HP1 accumulation at distinct nuclear foci has been associated with replication stress induced senescence ${ }^{47}$. Increased accumulation of H3K9me3 at these senescence-associated heterochromatin foci (SAHF) is the consequence of subnuclear redistribution rather than increased global $\mathrm{H} 3 \mathrm{~K} 9 \mathrm{me} 3$ levels ${ }^{48}$. We identified several indicators of a senescence-like response in Erg1-KD cultures pid. In addition, however, a global H3K9me3 increase at later time points $(9,15$ days) is observed. The elevated $\mathrm{H} 3 \mathrm{~K} 9 \mathrm{me} 3$ is preceded by increased KAP1 expression, which was reported to increase heterochromatin formation through recruitment of SETDB1 ${ }^{49}$. KAP1 is specifically required to resolve DNA damage in heterochromatin, down stream of ATM signaling ${ }^{50}$. As Kap1 and HP1 physically interact ${ }^{51}$, the elevated global H3K9me3 levels may point to increased heterochromatin formation. DNA damage in senescent cells was reported to persist for weeks on end ${ }^{52}$. As such H3K9me3 and Kap 1 may provide a means to protect damaged DNA from replication. 
Egr1 controls epigenetic reprogramming

Ezh2 levels were found to decrease in the context of replicative senescence ${ }^{53}$. Consistent with this, Ezh2 levels decrease during replicative stress in Egr1-KD cultures at 6 days pid. At late time points, elevated H3K27me3 correlates with increased Ezh2 expression. It is conceivable, that the formation of trimethylated chromatin represents a protective response against abnormal differentiation, cell death or transformation. Of note: all three trimethyl marks on histone 3 increase during transit amplification, suggesting a role under conditions of increased chromatin stress. In line with this notion, increased H3K4me3 and H3K27me3 have both been associated with other cellular stress responses: we and others have observed increased histone $3 \mathrm{~K} 4 \mathrm{me} 3$ and $\mathrm{K} 27 \mathrm{me} 3$ in response to hypoxia or conditions that evoke replication stress $\left({ }^{54}, \mathrm{FS}, \mathrm{PP}\right.$ and JWV; unpublished data). In addition, H3K4me3 was identified as a crucial determinant of Rag2-mediated V(D)J recombination in $B$ and $T$ cells, and is linked to DDR-induced cellular responses via ING family proteins ${ }^{55,56}$. These combined observations suggest roles for global trimethyl marking in processes in addition to local regulation of transcription.

Interestingly we observe potential mechanistic interactions of Egr1 with PRC function at several levels. Firstly, loss of Egr1 affects, directly or indirectly, expression of two important PRC members, Ezh2 and Bmi1. Both proteins have been associated with cell cycle regulation: Ezh2 is a direct target of E2F1 ${ }^{57}$, and Bmi1 controls expression of P16/INK4A and P14 ${ }^{\mathrm{ARF}}$, both encoded by the CDKN2/INK4A locus ${ }^{58,59}$. Secondly, comparative analysis of published PRC targets and a genome wide scan for Egr1 consensus binding sites yielded potential common targets and suggests coordinated regulation. Indeed we observe a close correlation between the presence of PRC-repressive H3K27me3 marks and lack of Egr1 enrichment 2 hours pid, suggesting that PRCs prevent Egr1 access to promoters. Interestingly, likewise, H3K27me3 prevents Myc from interacting with its genomic targets ${ }^{60}$. Importantly, we recently found that PRC1-LOF has comparable effects on chondrogenesis as Egr1-LOF, suggesting that at least part of the Egr1-LOF phenotype can be explained by PRC-LOF (FS, JWV: in preparation).

Finally, Egr1 was reported to be sumoylated in a $\mathrm{P} 14^{\mathrm{ARF}}$ mediated process ${ }^{61}$. The responsible E3 ligase, UBC9, also interacts with PRC1 \& $2{ }^{62,63}$. Egr1-sumoylation is required for transcriptional activation of the Egr1-target PTEN. The protein phosphatase PTEN deactivates PI3K, which in turn will no longer activate AKT. In our experimental setting, however, we observe no induction of PTEN mRNA and Egr1-KD only marginally affects PTEN expression. This would suggest, that either most regulation occurs at the level of post-translational modification or that the EGR1-PTENAKT-INK4AARF axis may not be a prime regulatory module early in chondrogenesis. In line with cell context dependent responses, several studies report cell type-dependent responses an Egr1targeted reporter gene was activated in neural cells, whereas in cartilage and heart it was suppressed $^{64,65}$.

Epigenomic reprogramming by Egr1 may serve to define concerted transcriptional or replication activity of gene-networks to guide cells through chondrogenesis. In agreement with such a coordinating role, Egr1 is known to transactivate and recruit CBP/P300, the consequence of which 


\section{Chapter 4}

is increased local HAT activity ${ }^{66}$. In contrast, Egr1 interaction with NAB1/2 and recruitment of the NuRD complex would repress transcription by bringing HDAC activity to target genes ${ }^{67-69}$. The global distribution of Egr1 binding sites may point to a more general task in epigenomic reprogramming, not exclusively linked to transcription. By analogy, recent studies on genomic distribution of transcription factor (TF) binding sites (PPARy, ERa) identified up to half of such binding sites either in intragenic regions (introns) or at distant locations ( $>25 \mathrm{~kb}$ away from the nearest gene), suggest additional epigenomic roles besides TF binding in gene promoters ${ }^{70-72}$. It is tempting to hypothesize a role for IEGs in early epigenomic pre-programming, such that ensuing processes (concurrent transcription and replication) are facilitated in the context of development. Several observations support a close relationship between epigenetic marking and changed DNA replication during differentiation. Replication of lineage-relevant and non-relevant genes appears functionally separated in time: relevant genes ('open' chromatin ${ }^{73,74}$ ) is replicated earlier in Sphase than non-lineage related genes. Dynamic change of replication timing throughout differentiation is crucial, and regulation of these processes is provided by the micro-environment of a stem cell or progenitor. Although still far from completely understood, translation of this epigenomic 'landscape' ${ }^{75}$ provides the 'traffic signs and road maps' to terminally differentiated cell fates. It will be of considerable interest to map global and local acetylation in respect to Egr1 binding and replication activity. Likewise, correlation of K4, K9 and K27 trimethyl marks, and likely numerous additional epigenetic marks, in the ATDC5 Egr1-KD system to DNA-templated activity will help advance insight into epigenetic regulation of the genome. These experiments are currently underway.

Although epigenetic mechanisms in differentiation begin to emerge, exact knowledge of how cells communicate environmental changes to chromatin and how global epigenomic remodeling accompanies differentiation is lacking. At the onset of the study reported here, knowledge on IEGs in the context of chondrogenesis was limited. A general loss of Egr1 expression was reported in degenerating human cartilage ${ }^{76}$. Although EGR family members may regulate each others expression ${ }^{77}$ several studies provide evidence for distinctive functions for individual Egr1 family members. In adipogenesis, Egr1 and Egr2 have different roles ${ }^{78}$. In keeping with this, the here reported strong phenotypic response to Egr1-LOF, despite induction of other closely related Egrfamiliy IEGs, point to absence of functional compensation and argue for a unique role for EGR1 in early chondrogenesis: the acute nature of an RNAi mediated knock-down approach used in this study, may prevent activation of redundant mechanisms often observed in null-mutant models ${ }^{79}$. Here, we report a dual function for the transcription factor Egr1 in activating a lineage-specific transcriptional re-programming, as well as transit progenitor expansion. The inappropriate chromatin reprogramming in the absence of Egr1 strongly support an important task for Egr1 in early epigenomic remodeling during chondrogenesis and pave the way for further studies in geneenvironment interactions. 
Table 1: Genmapp overrepresentation table (database used: Mm_Derby_20090509.pgdb; processes with a $Z$ score $>1.96$ are presented).

\begin{tabular}{|c|c|c|c|c|c|}
\hline Pathway & positive (r) & $\begin{array}{l}\text { measured } \\
\text { (n) }\end{array}$ & total & $\%$ & Z Score \\
\hline Insulin Signaling & 46 & 156 & 160 & $29,49 \%$ & 5,10 \\
\hline EGFR1 Signaling Pathway & 48 & 171 & 172 & $28,07 \%$ & 4,83 \\
\hline IL -4 signaling Pathway & 21 & 58 & 60 & $36,21 \%$ & 4,50 \\
\hline Regulation of Actin Cytoskeleton & 37 & 146 & 150 & $25,34 \%$ & 3,50 \\
\hline G Protein Signaling Pathways & 24 & 84 & 87 & $28,57 \%$ & 3,47 \\
\hline IL -6 signaling Pathway & 26 & 96 & 97 & $27,08 \%$ & 3,30 \\
\hline Wnt Signaling Pathway NetPath & 28 & 107 & 109 & $26,17 \%$ & 3,22 \\
\hline Calcium Regulation in the Cardiac Cell & 36 & 148 & 151 & $24,32 \%$ & 3,17 \\
\hline IL-2 Signaling Pathway & 20 & 73 & 74 & $27,40 \%$ & 2,94 \\
\hline IL -5 Signaling Pathway & 18 & 65 & 66 & $27,69 \%$ & 2,84 \\
\hline miR-1 in cardiac development & 3 & 5 & 5 & $60,00 \%$ & 2,80 \\
\hline IL_-3 Signaling Pathway & 24 & 95 & 97 & $25,26 \%$ & 2,78 \\
\hline Kit Receptor Signaling Pathway & 17 & 65 & 66 & $26,15 \%$ & 2,49 \\
\hline Heme Biosynthesis & 4 & 9 & 9 & $44,44 \%$ & 2,45 \\
\hline MAPK Cascade & 9 & 29 & 30 & $31,03 \%$ & 2,39 \\
\hline Nuclear Receptors & 11 & 38 & 38 & $28,95 \%$ & 2,38 \\
\hline Mouse ESC Pluripotency Pathways & 25 & 109 & 112 & $22,94 \%$ & 2,30 \\
\hline Proteasome Degradation & 19 & 78 & 81 & $24,36 \%$ & 2,29 \\
\hline Circadian Exersice & 13 & 49 & 49 & $26,53 \%$ & 2,23 \\
\hline Circadian Exersice & 13 & 49 & 49 & $26,53 \%$ & 2,23 \\
\hline Id Signaling Pathway & 13 & 49 & 51 & $26,53 \%$ & 2,23 \\
\hline Myometrial Relaxation and Contraction & 34 & 160 & 161 & $21,25 \%$ & 2,20 \\
\hline MAPK signaling pathway & 29 & 134 & 138 & $21,64 \%$ & 2,13 \\
\hline Wnt Signaling Pathway & 15 & 61 & 62 & $24,59 \%$ & 2,07 \\
\hline IL-9 Signaling Pathway & 7 & 23 & 24 & $30,43 \%$ & 2,05 \\
\hline IL-1 Signaling Pathway & 10 & 37 & 38 & $27,03 \%$ & 2,02 \\
\hline IL-7 Signaling Pathway & 11 & 42 & 44 & $26,19 \%$ & 2,00 \\
\hline p38 MAPK Signaling Pathway (BioCarta) & 8 & 28 & 30 & $28,57 \%$ & 1,98 \\
\hline
\end{tabular}

\section{Acknowledgements}

The authors thank B. Wouters (Toronto, Canada) and M Starmans (Maastricht, The Netherlands) for critically reading the manuscript. The authors received financial support from the European Molecular Biology Organization (Germany) ASTF5-2009; the Dutch Science Organization (ZonMW-NWO) VIDI grant 016.046.362, the National Rheuma Foundation (Reumafonds) grant LLP14 and transnational University of Limburg (tUL) grant to JWV. 


\section{References}

1. Jenuwein, T. \& Allis, C.D. Translating the histone code. Science 293, 1074-1080 (2001).

2. Berger, S.L. The complex language of chromatin regulation during transcription. Nature 447, 407412 (2007).

3. Rando, O.J. Global patterns of histone modifications. Curr Opin Genet Dev 17, 94-99 (2007).

4. Bernstein, B.E. et al. A bivalent chromatin structure marks key developmental genes in embryonic stem cells. Cell 125, 315-326 (2006).

5. Burdan, F. et al. Morphology and physiology of the epiphyseal growth plate. Folia Histochem Cytobiol 47, 5-16 (2009).

6. Koay, E.J. \& Athanasiou, K.A. Hypoxic chondrogenic differentiation of human embryonic stem cells enhances cartilage protein synthesis and biomechanical functionality. Osteoarthritis Cartilage 16, 1450-1456 (2008).

7. Liu, F., Kohlmeier, S. \& Wang, C.Y. Wnt signaling and skeletal development. Cell Signal 20, 9991009 (2008).

8. Goldring, M.B., Tsuchimochi, K. \& ljiri, K. The control of chondrogenesis. J Cell Biochem 97, 33-44 (2006).

9. Kuzmichev, A., Nishioka, K., Erdjument-Bromage, H., Tempst, P. \& Reinberg, D. Histone methyltransferase activity associated with a human multiprotein complex containing the Enhancer of Zeste protein. Genes Dev 16, 2893-2905 (2002).

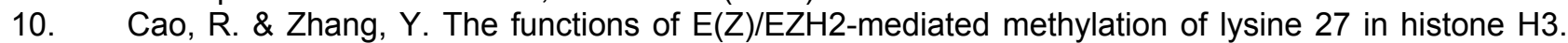
Curr Opin Genet Dev 14, 155-164 (2004).

11. Bracken, A.P., Dietrich, N., Pasini, D., Hansen, K.H. \& Helin, K. Genome-wide mapping of Polycomb target genes unravels their roles in cell fate transitions. Genes Dev 20, 1123-1136 (2006).

12. Hemenway, C.S., Halligan, B.W. \& Levy, L.S. The Bmi-1 oncoprotein interacts with dinG and MPh2: the role of RING finger domains. Oncogene 16, 2541-2547 (1998).

13. Akasaka, T. et al. A role for mel-18, a Polycomb group-related vertebrate gene, during theanteroposterior specification of the axial skeleton. Development 122, 1513-1522 (1996).

14. Suzuki, M. et al. Involvement of the Polycomb-group gene Ring1B in the specification of the anteriorposterior axis in mice. Development 129, 4171-4183 (2002).

15. van der Lugt, N.M., Alkema, M., Berns, A. \& Deschamps, J. The Polycomb-group homolog Bmi-1 is a regulator of murine Hox gene expression. Mech Dev 58, 153-164 (1996).

16. Wang, J., Mager, J., Schnedier, E. \& Magnuson, T. The mouse PcG gene eed is required for Hox gene repression and extraembryonic development. Mamm Genome 13, 493-503 (2002).

17. Mackie, E.J., Ahmed, Y.A., Tatarczuch, L., Chen, K.S. \& Mirams, M. Endochondral ossification: How cartilage is converted into bone in the developing skeleton. Int J Biochem Cell Biol 40, 46-62 (2008).

18. Nilsson, O., Marino, R., De Luca, F., Phillip, M. \& Baron, J. Endocrine regulation of the growth plate. Horm Res 64, 157-165 (2005).

19. Wu, X., Shi, W. \& Cao, X. Multiplicity of BMP signaling in skeletal development. Ann N Y Acad Sci 1116, 29-49 (2007).

20. Hutchison, M.R., Bassett, M.H. \& White, P.C. Insulin-like growth factor-I and fibroblast growth factor, but not growth hormone, affect growth plate chondrocyte proliferation. Endocrinology 148, 31223130 (2007).

21. Atsumi, T., Miwa, Y., Kimata, K. \& Ikawa, Y. A chondrogenic cell line derived from a differentiating culture of AT805 teratocarcinoma cells. Cell Differ Dev 30, 109-116 (1990).

22. Fujii, M. et al. Roles of bone morphogenetic protein type I receptors and Smad proteins in osteoblast and chondroblast differentiation. Mol Biol Cell 10, 3801-3813 (1999).

23. Shukunami, C. et al. Chondrogenic differentiation of clonal mouse embryonic cell line ATDC5 in vitro: differentiation-dependent gene expression of parathyroid hormone (PTH)/PTH-related peptide receptor. J Cell Biol 133, 457-468 (1996).

24. Abu-Shakra, S.R., Cole, A.J. \& Drachman, D.B. Nerve stimulation and denervation induce differential patterns of immediate early gene mRNA expression in skeletal muscle. Brain Res Mol Brain Res 18, 216-220 (1993).

25. Carter, D.A. Neurotransmitter-stimulated immediate-early gene responses are organized through differential post-synaptic receptor mechanisms. Brain Res Mol Brain Res 16, 111-118 (1992).

26. Baron, V., Adamson, E.D., Calogero, A., Ragona, G. \& Mercola, D. The transcription factor Egr1 is a direct regulator of multiple tumor suppressors including TGFbeta1, PTEN, p53, and fibronectin. Cancer Gene Ther 13, 115-124 (2006).

27. Cao, X.M. et al. Identification and characterization of the Egr-1 gene product, a DNA-binding zinc finger protein induced by differentiation and growth signals. Mol Cell Biol 10, 1931-1939 (1990).

28. Gashler, A. \& Sukhatme, V.P. Early growth response protein 1 (Egr-1): prototype of a zinc-finger family of transcription factors. Prog Nucleic Acid Res Mol Biol 50, 191-224 (1995). 
29. Swirnoff, A.H. \& Milbrandt, J. DNA-binding specificity of NGFI-A and related zinc finger transcription factors. Mol Cell Biol 15, 2275-2287 (1995).

30. Smith, N. et al. Overlapping expression of Runx1(Cbfa2) and Runx2(Cbfa1) transcription factors supports cooperative induction of skeletal development. J Cell Physiol 203, 133-143 (2005).

31. Stricker, S., Fundele, R., Vortkamp, A. \& Mundlos, S. Role of Runx genes in chondrocyte differentiation. Dev Biol 245, 95-108 (2002).

32. Akiyama, H., Chaboissier, M.C., Martin, J.F., Schedl, A. \& de Crombrugghe, B. The transcription factor Sox9 has essential roles in successive steps of the chondrocyte differentiation pathway and is required for expression of Sox5 and Sox6. Genes Dev 16, 2813-2828 (2002).

33. Bell, D.M. et al. SOX9 directly regulates the type-Il collagen gene. Nat Genet 16, 174-178 (1997).

34. Brummelkamp, T.R., Bernards, R. \& Agami, R. A system for stable expression of short interfering RNAs in mammalian cells. Science 296, 550-553 (2002).

35. Zheng, Q. et al. Type X collagen gene regulation by Runx2 contributes directly to its hypertrophic chondrocyte-specific expression in vivo. J Cell Biol 162, 833-842 (2003).

36. Hong, D.S., Angelo, L.S. \& Kurzrock, R. Interleukin-6 and its receptor in cancer: implications for Translational Therapeutics. Cancer 110, 1911-1928 (2007).

37. Kuilman, T. et al. Oncogene-induced senescence relayed by an interleukin-dependent inflammatory network. Cell 133, 1019-1031 (2008).

38. Deschamps, J. Developmental biology. Hox genes in the limb: a play in two acts. Science 304, 1610-1611 (2004).

39. Deschamps, J. et al. Initiation, establishment and maintenance of Hox gene expression patterns in the mouse. Int J Dev Biol 43, 635-650. (1999).

40. Soshnikova, N. \& Duboule, D. Epigenetic temporal control of mouse Hox genes in vivo. Science 324, 1320-1323 (2009).

41. Duboule, D. \& Morata, G. Colinearity and functional hierarchy among genes of the homeotic complexes. Trends Genet 10, 358-364 (1994).

42. Courtois-Cox, S. et al. A negative feedback signaling network underlies oncogene-induced senescence. Cancer Cell 10, 459-472 (2006).

43. Di Micco, R. et al. Oncogene-induced senescence is a DNA damage response triggered by DNA hyper-replication. Nature 444, 638-642 (2006).

44. Mallette, F.A., Gaumont-Leclerc, M.F. \& Ferbeyre, G. The DNA damage signaling pathway is a critical mediator of oncogene-induced senescence. Genes Dev 21, 43-48 (2007).

45. Thorne, J.L., Campbell, M.J. \& Turner, B.M. Transcription factors, chromatin and cancer. Int $J$ Biochem Cell Biol 41, 164-175 (2009).

46. Knoepfler, P.S. et al. Myc influences global chromatin structure. Embo J 25, 2723-2734 (2006).

47. Narita, M. et al. Rb-mediated heterochromatin formation and silencing of E2F target genes during cellular senescence. Cell 113, 703-716 (2003).

48. Grewal, S.I. \& Jia, S. Heterochromatin revisited. Nat Rev Genet 8, 35-46 (2007).

49. Schultz, D.C., Ayyanathan, K., Negorev, D., Maul, G.G. \& Rauscher, F.J., III SETDB1: a novel KAP1-associated histone $\mathrm{H} 3$, lysine 9-specific methyltransferase that contributes to HP1-mediated silencing of euchromatic genes by KRAB zinc-finger proteins. Genes Dev. 16, 919-932 (2002).

50. Goodarzi, A.A. et al. ATM Signaling Facilitates Repair of DNA Double-Strand Breaks Associated with Heterochromatin. Molecular Cell 31, 167-177 (2008).

51. Ryan, R.F. et al. KAP-1 corepressor protein interacts and colocalizes with heterochromatic and euchromatic HP1 proteins: a potential role for Kruppel-associated box-zinc finger proteins in heterochromatin-mediated gene silencing. Mol Cell Biol 19, 4366-4378 (1999).

52. Rodier, F. et al. Persistent DNA damage signalling triggers senescence-associated inflammatory cytokine secretion. Nat Cell Biol (2009).

53. Bracken, A.P. et al. The Polycomb group proteins bind throughout the INK4A-ARF locus and are disassociated in senescent cells. Genes Dev 21, 525-530 (2007).

54. Johnson, A.B., Denko, N. \& Barton, M.C. Hypoxia induces a novel signature of chromatin modifications and global repression of transcription. Mutat Res (2008).

55. Matthews, A.G. et al. RAG2 PHD finger couples histone H3 lysine 4 trimethylation with V(D)J recombination. Nature 450, 1106-1110 (2007).

56. Shi, X. et al. ING2 PHD domain links histone H3 lysine 4 methylation to active gene repression. Nature 442, 96-99 (2006).

57. Bracken, A.P. et al. EZH2 is downstream of the pRB-E2F pathway, essential for proliferation and amplified in cancer. Embo J 22, 5323-5335 (2003).

58. Jacobs, J.J., Kieboom, K., Marino, S., DePinho, R.A. \& van Lohuizen, M. The oncogene and Polycomb-group gene bmi-1 regulates cell proliferation and senescence through the ink4a locus. Nature 397, 164-168 (1999).

59. Jacobs, J.J. et al. Bmi-1 collaborates with c-Myc in tumorigenesis by inhibiting c-Myc- induced apoptosis via INK4a/ARF. Genes Dev. 13, 2678-2690 (1999). 
60. Goodliffe, J.M., Wieschaus, E. \& Cole, M.D. Polycomb mediates Myc autorepression and its transcriptional control of many loci in Drosophila. Genes Dev 19, 2941-2946 (2005).

61. $\mathrm{Yu}$, J. et al. PTEN regulation by Akt-EGR1-ARF-PTEN axis. Embo J 28, 21-33 (2009).

62. Zhang, J. \& Sarge, K.D. Mel-18 interacts with RanGAP1 and inhibits its sumoylation. Biochemical and Biophysical Research Communications 375, 252-255 (2008).

63. Riising, E.M., Boggio, R., Chiocca, S., Helin, K. \& Pasini, D. The Polycomb Repressive Complex 2 Is a Potential Target of SUMO Modifications. PLOS ONE 3, e2704 (2008).

64. Tan, L. et al. Egr-1 mediates transcriptional repression of COL2A1 promoter activity by interleukin1beta. J Biol Chem 278, 17688-17700 (2003).

65. Erno, H., Kury, P., Botteri, F.M. \& Monard, D. A Krox binding site regulates protease nexin-1 promoter activity in embryonic heart, cartilage and parts of the nervous system. Mech Dev 60, 139150 (1996).

66. Silverman, E.S. et al. cAMP-response-element-binding-protein-binding protein (CBP) and p300 are transcriptional co-activators of early growth response factor-1 (Egr-1). Biochem J 336 ( Pt 1), 183189 (1998).

67. Svaren, J. et al. NAB2, a corepressor of NGFI-A (Egr-1) and Krox20, is induced by proliferative and differentiative stimuli. Mol Cell Biol 16, 3545-3553 (1996).

68. Thiel, G. et al. The human transcriptional repressor protein NAB1: expression and biological activity. Biochim Biophys Acta 1493, 289-301 (2000).

69. Srinivasan, R., Mager, G.M., Ward, R.M., Mayer, J. \& Svaren, J. NAB2 represses transcription by interacting with the CHD4 subunit of the nucleosome remodeling and deacetylase (NuRD) complex. J Biol Chem 281, 15129-15137 (2006).

70. Nielsen, R. et al. Genome-wide profiling of PPARgamma:RXR and RNA polymerase II occupancy reveals temporal activation of distinct metabolic pathways and changes in RXR dimer composition during adipogenesis. Genes Dev 22, 2953-2967 (2008).

71. Welboren, W.-J. et al. ChIP-Seq of ER[alpha] and RNA polymerase II defines genes differentially responding to ligands. EMBO J 28, 1418-1428 (2009).

72. Carroll, J.S. et al. Genome-wide analysis of estrogen receptor binding sites. Nat Genet 38, 12891297 (2006).

73. Gilbert, D.M. Replication timing and transcriptional control: beyond cause and effect. Curr Opin Cell Biol 14, 377-383 (2002).

74. Hiratani, I., Takebayashi, S., Lu, J. \& Gilbert, D.M. Replication timing and transcriptional control: beyond cause and effect--part II. Curr Opin Genet Dev 19, 142-149 (2009).

75. Holliday, R. Epigenetics: a historical overview. Epigenetics 1, 76-80 (2006).

76. Wang, F.L. et al. Differential expression of egr-1 in osteoarthritic compared to normal adult human articular cartilage. Osteoarthritis Cartilage 8, 161-169 (2000).

77. Nagarajan, R. et al. EGR2 mutations in inherited neuropathies dominant-negatively inhibit myelin gene expression. Neuron 30, 355-368 (2001).

78. Boyle, K.B. et al. The transcription factors Egr1 and Egr2 have opposing influences on adipocyte differentiation. Cell Death Differ 16, 782-789 (2009).

79. Carter, J.H. \& Tourtellotte, W.G. Early growth response transcriptional regulators are dispensable for macrophage differentiation. J Immunol 178, 3038-3047 (2007).

80. R Development Core Team (2008). R: A language and environment for statistical computing. $R$ Foundation for Statistical Computing, Vienna, Austria. ISBN 3-900051-07-0,URL http://www.Rproject.org.

81. PMID: 15461798 - url: http://www.bioconductor.org/

82. PMID: 15175677 
A
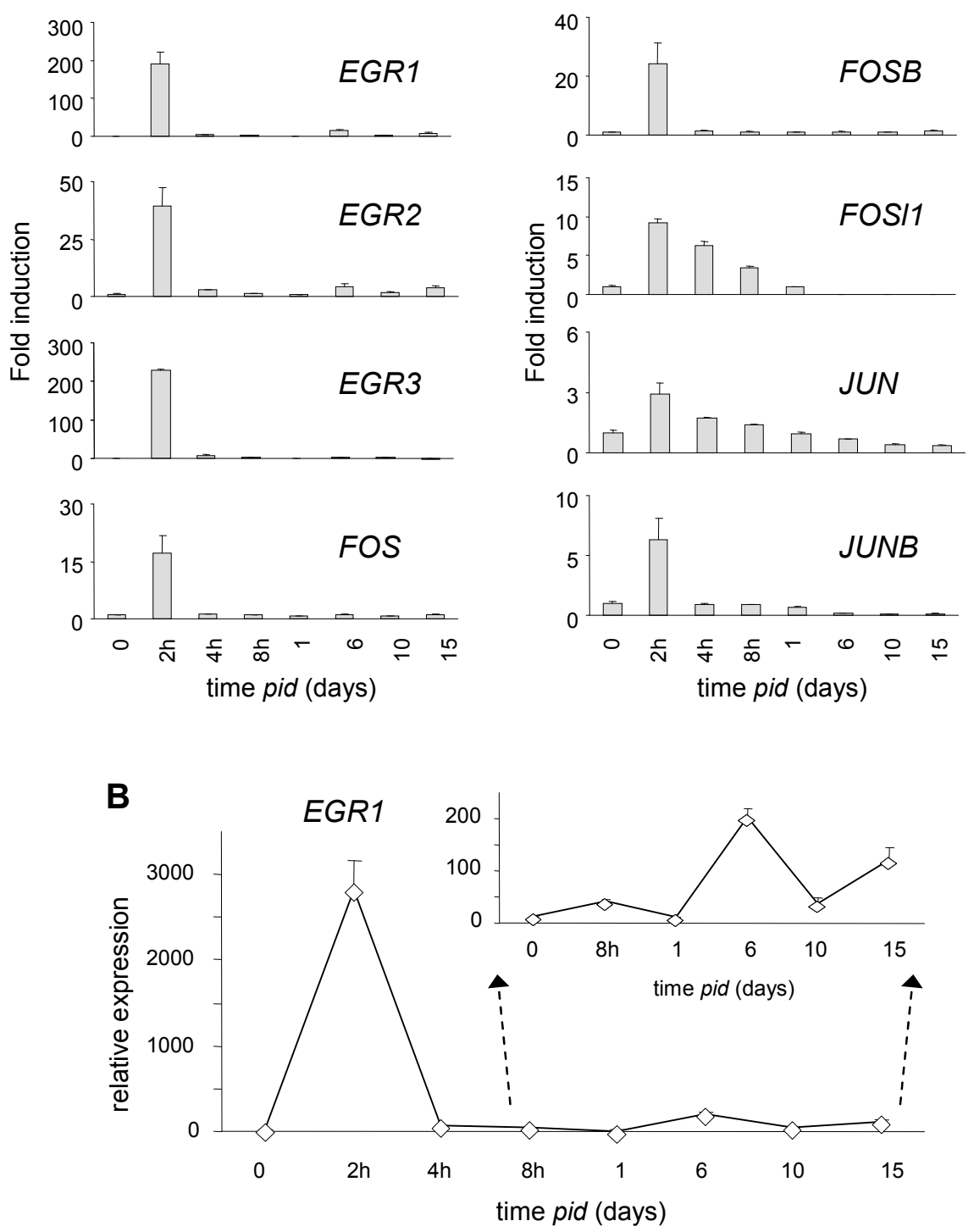

Figure S1. Expression profiles Inmediate Early Genes throughout chondrogenesis.

$(A, B)$ Microarray analysis of Immediate Early Genes mRNA expression in ATDC5 chondrogenic differentation presented as fold induction compared to $T=0(A)$, or arbitrary units $(B)$. 


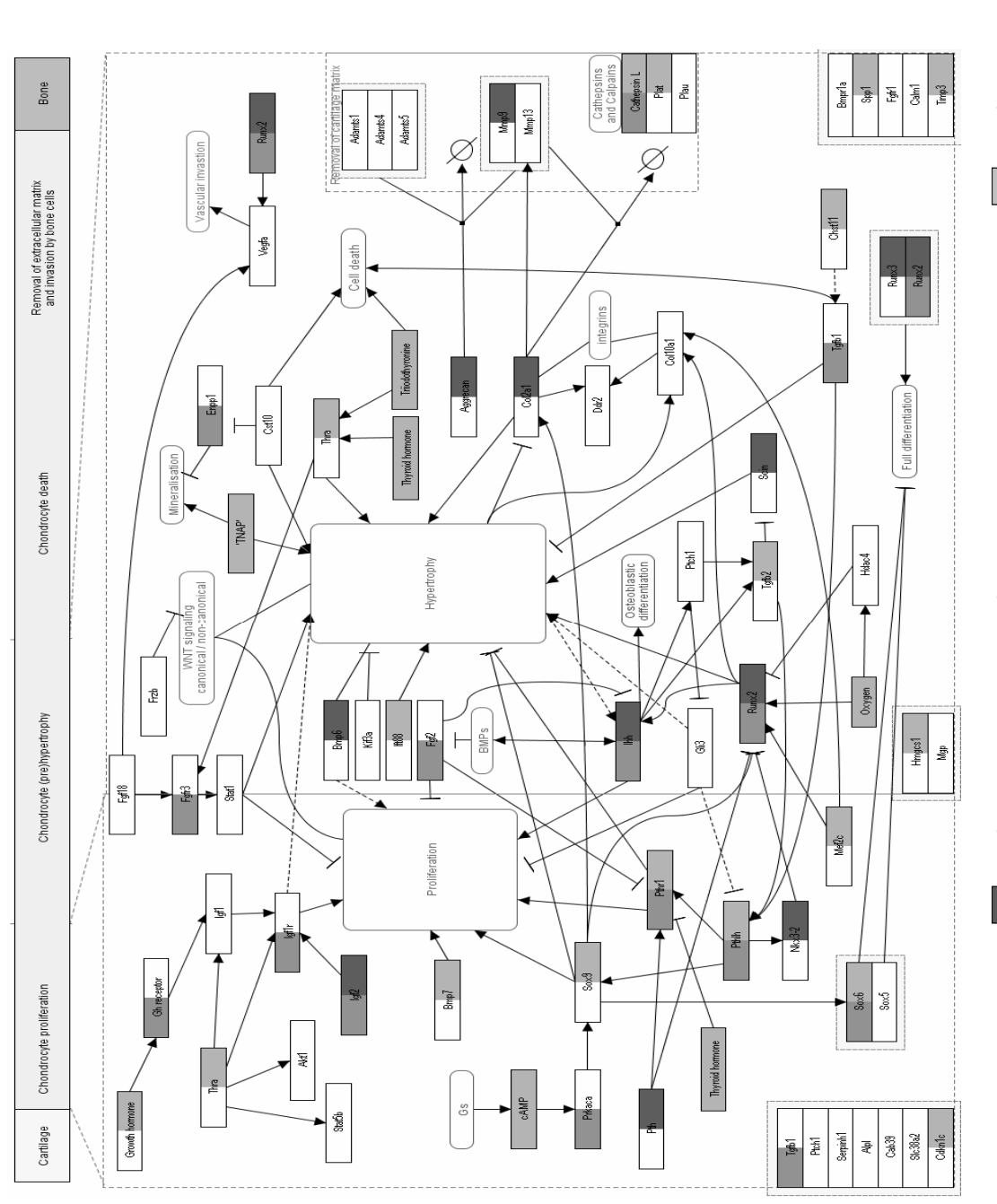

Figure S2. Enchondral ossification pathway analysis for predicted Egr1 targets and published PcG targets (Bracken et al.)

A genome-wide blast search was performed for Egr1 consensus binding sequences. The search was confined to GCGG/TGGGCG motifs and its reverse complementary sequence, both in sense and antisense orientation. Genes containing predicted binding sites were mapped using pathway analysis tools (Pathvisio with Wikipathways content). 


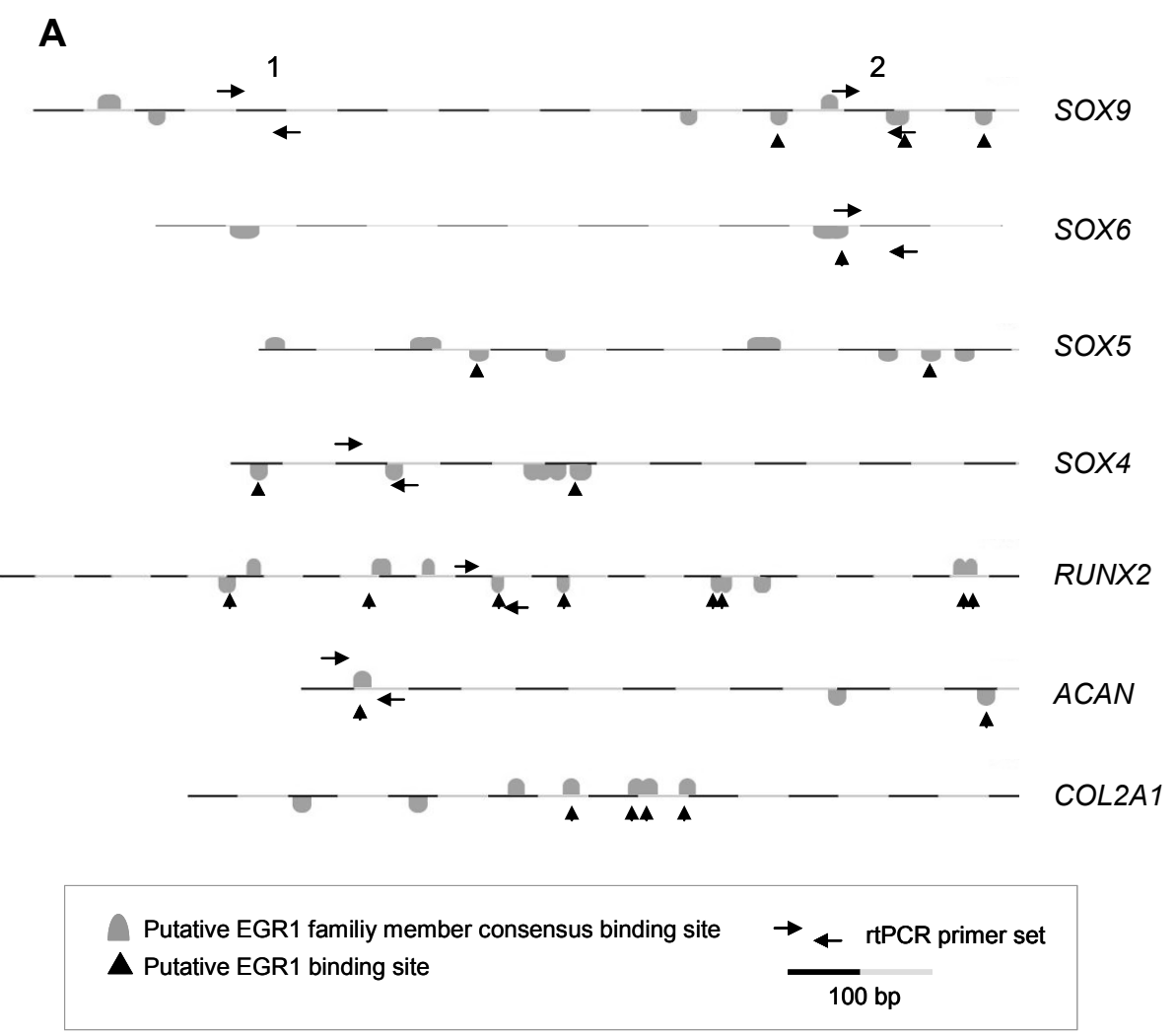

Figure S3. Genes involved in chondrogenesis contain multiple Egr1 consensus binding sites.

Using GENOMATIX promoter analysis software, promoters were analyzed for Egr1 binding sites. Arrows indicate primers designed for qRT rtPCR to quantify immunoprecipitated chromatin. 


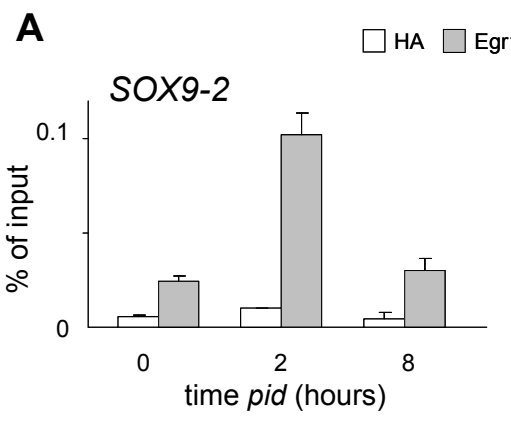

C
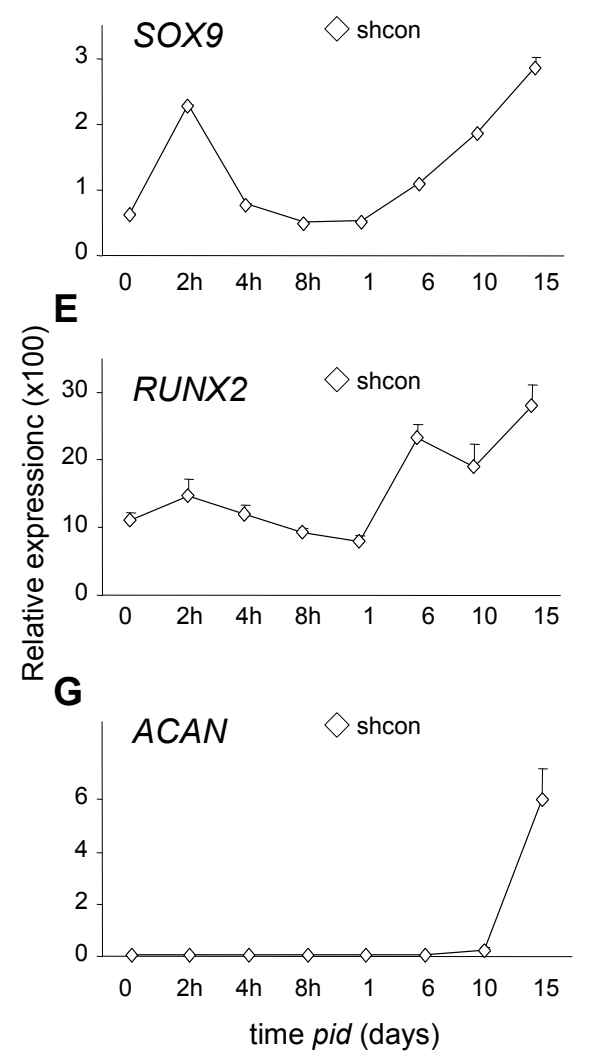

B $\square$ HA $\square$ H3K27me3 $\square$ H3K4me3

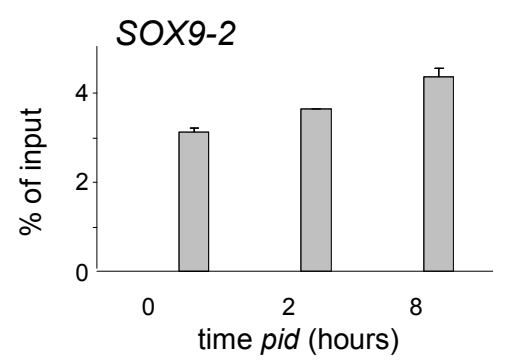

D
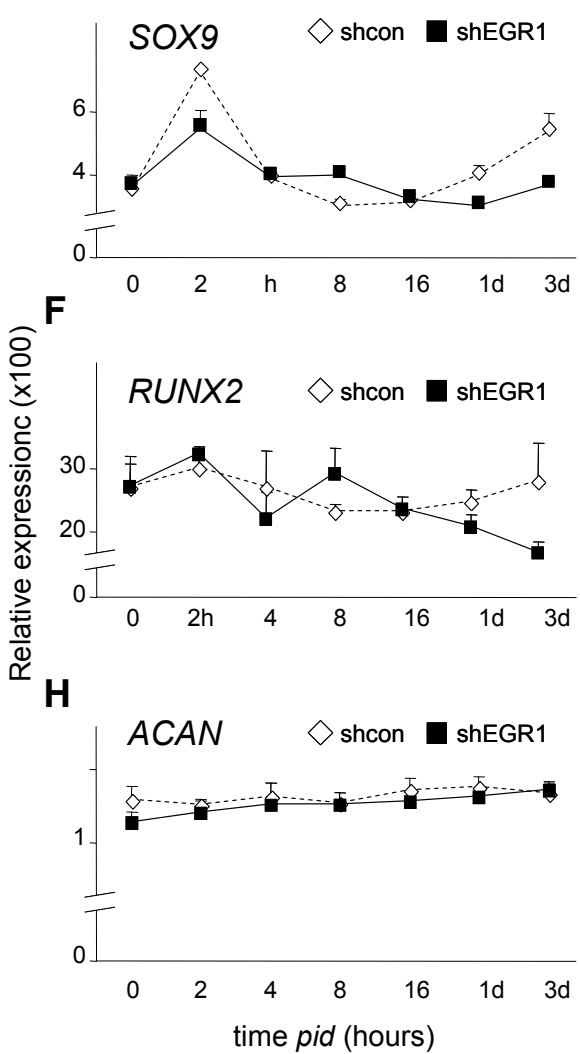

Figure S4. Egr1 directly binds and regulates the Sox9 promoter.

$(A, B)$ Chromatin immunoprecipitation using anti-Egr1, anti-H3K27me3 and anti-H3K4me3 antibodies. Precipitated DNA was analyzed by gRT rtPCR for enrichment on the Sox9 promoter using primer set 2 (see figure S3). (C-H) Microarray mRNA expression analysis of chondrogenic genes in ATDC5 cells stably expressing shControl or shEgr1 throughout chondrogenesis. 
A

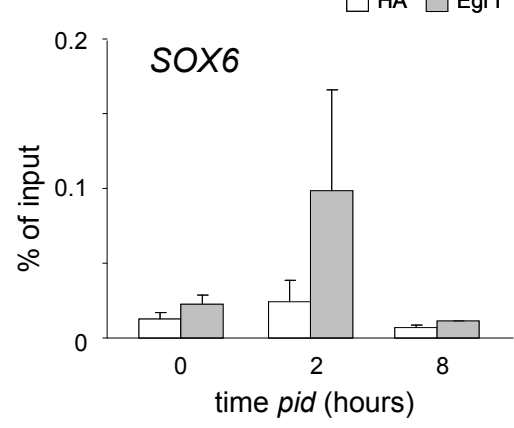

C

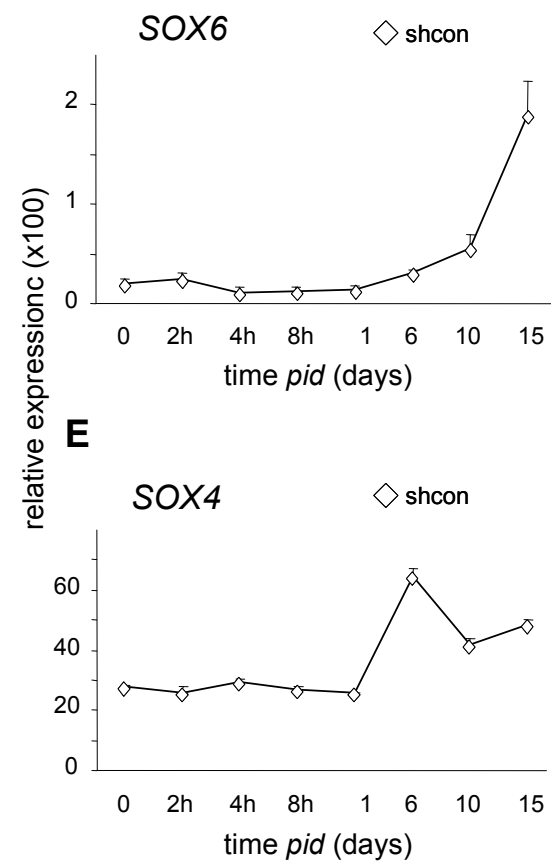

B $\square$ HA $\square$ H3K27me3 $\square$ H3K4me3

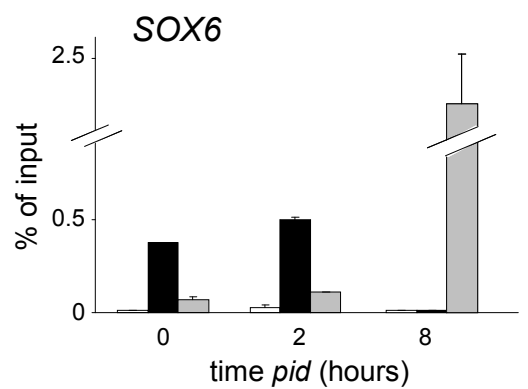

D

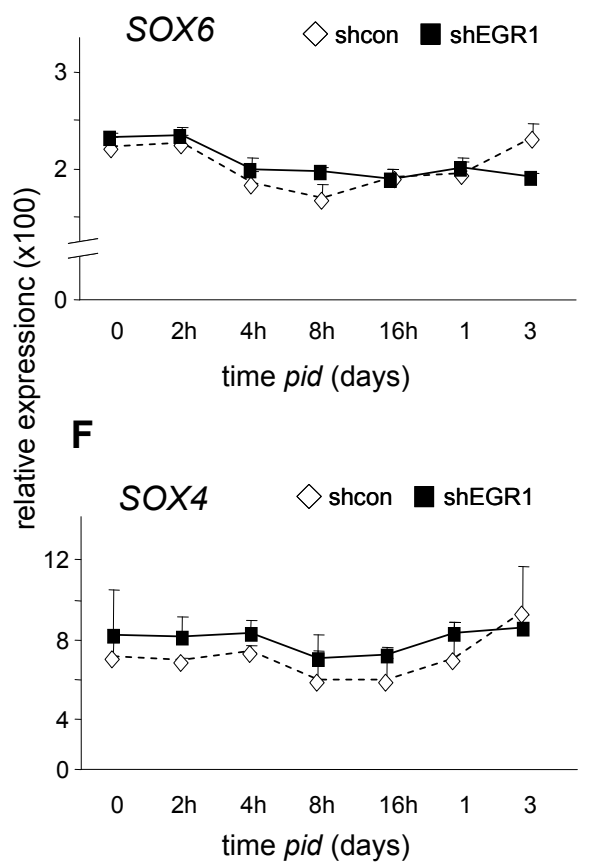

Figure S5. Egr1 directly binds and regulates the Sox6 promoter

$(A, B)$ Chromatin immunoprecipitation using anti-Egr1, anti-H3K27me3 and anti-H3K4me3 antibodies. Precipitated DNA was analyzed by gRT rtPCR for enrichment on the Sox6 promoter. (C-F) Microarray mRNA expression analysis of Sox6 and Sox4 in ATDC5 cells stably expressing shControl or shEgr1 throughout chondrogenesis. 


\section{Chapter 4}
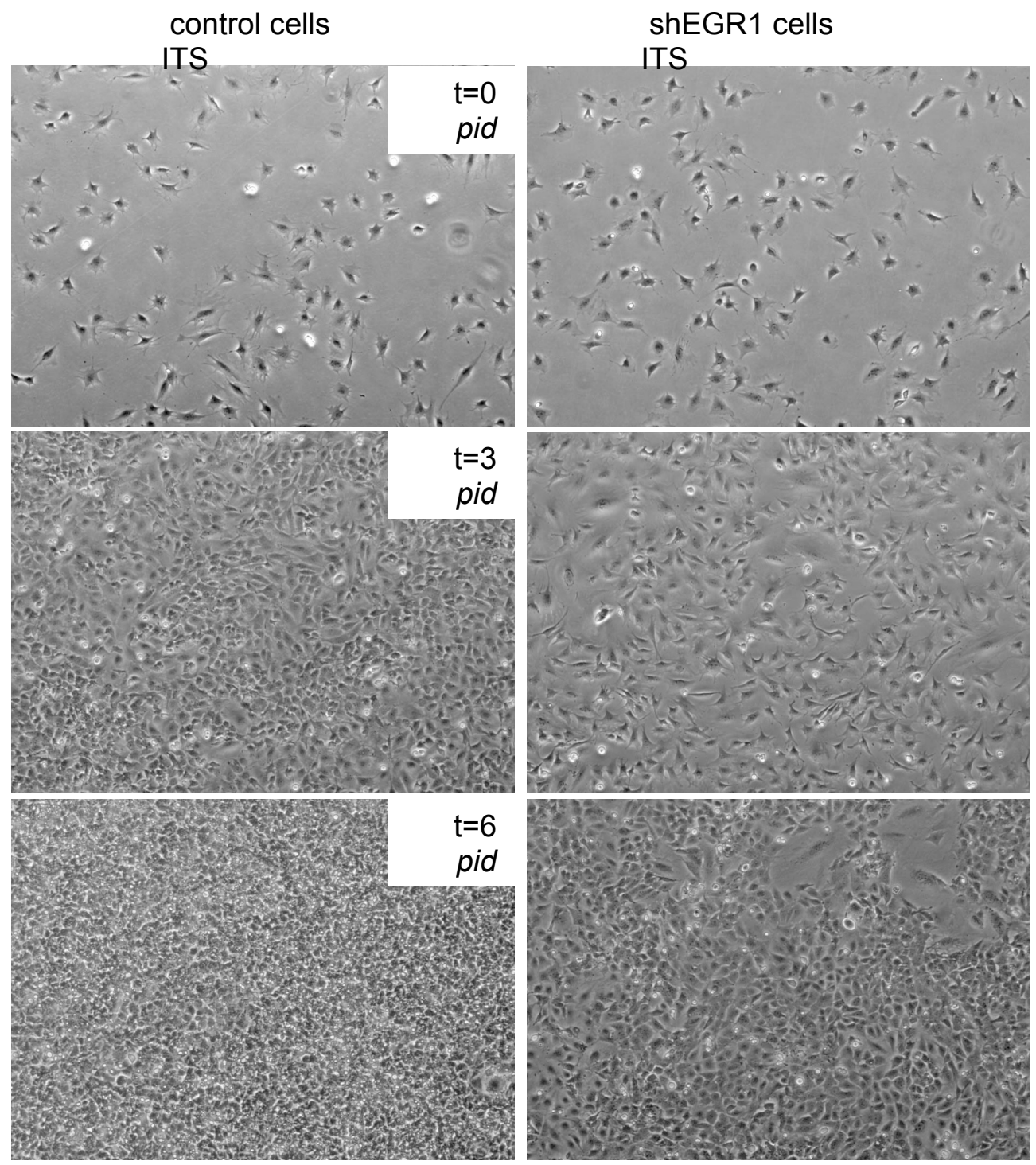

Figure S6. Loss of Egr1 affects differentiation induced hyperproliferation

Morphology analysis of ATDC5 stably expressing shControl or shEgr1 during the first 6 days of chondrogenesis. 


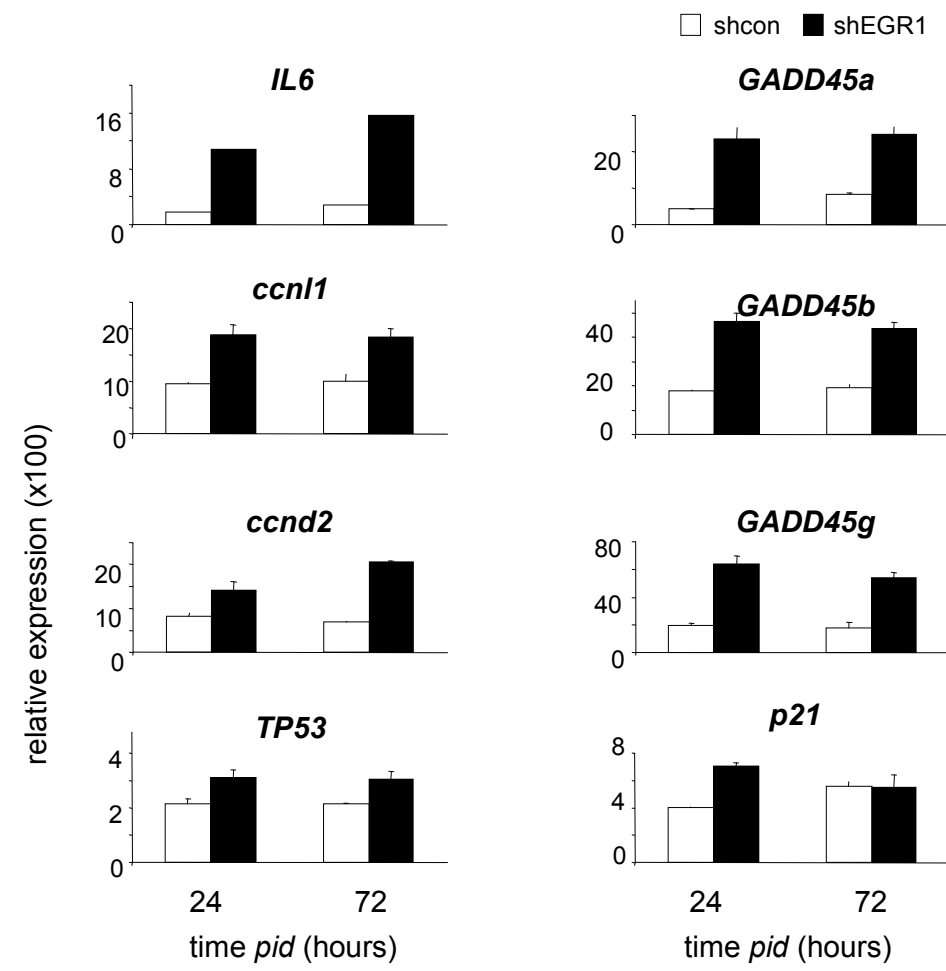

Figure S7. Loss of Egr1 induces numerous genes implicated in cellular senescence, DNA damage and stress signaling. Microarray mRNA expression analysis of genes involved in cellular senescence, DNA damage and stress signalling in ATDC 5 cells stably expressing shControl or shEgr1 throughout chondrogenesis. 
A

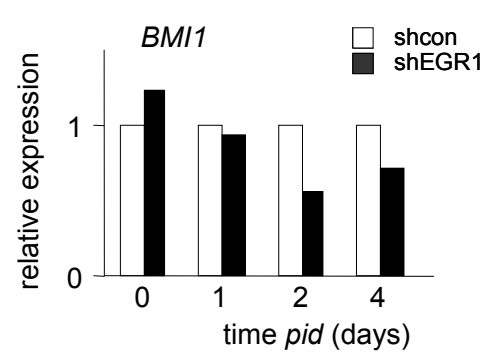

C

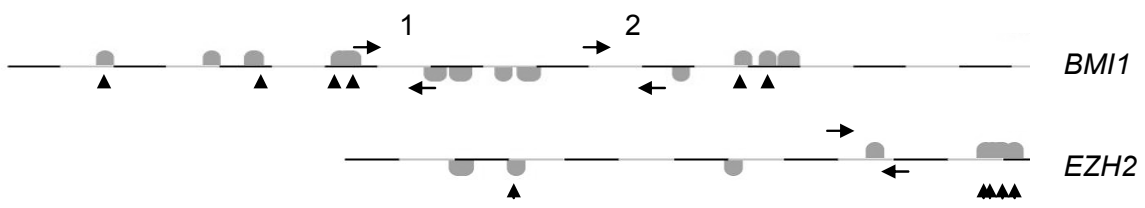

D

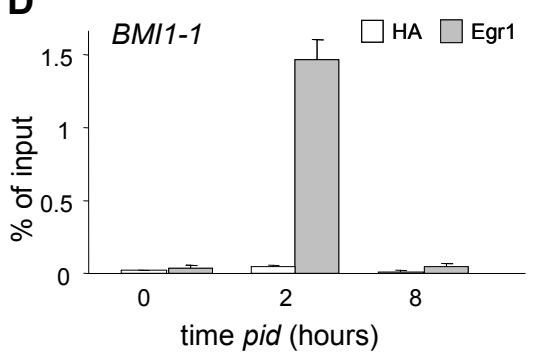

$\mathbf{F}$

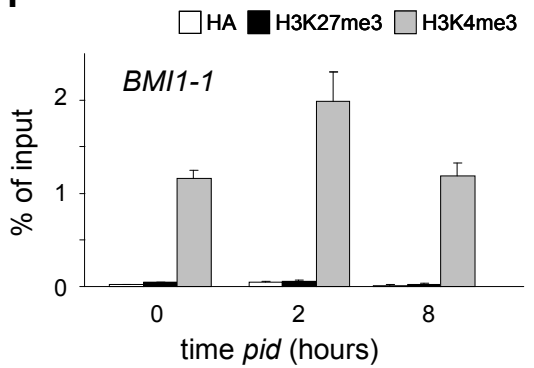

B

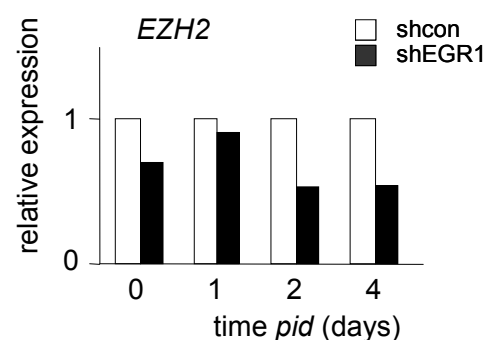

E

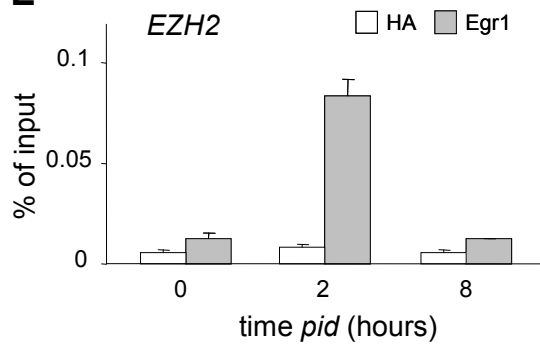

G

$\square$ HA $\square$ H3K27me3 $\square$ H3K4me3

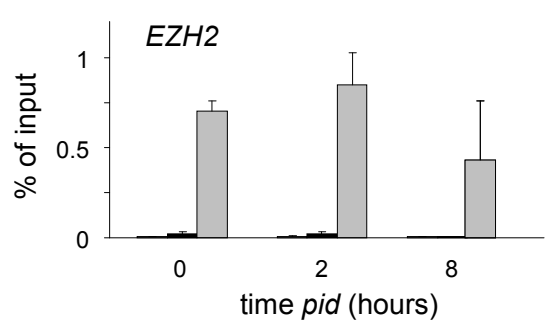

Figure S8. Egr1 directly binds PcG promoters and regulates PcG expression.

$(A, B)$ Relative mRNA expression of Bmi1 and Ezh2 in ATDC5 stably expressing shControl and shEgr1 up to 4 days pid. (C) using GENOMATIX promoter analysis software, Bmi1 and Ezh2 promoters were analyzed for Egr1 binding sites. Arrows indicate primers designed for qRT rtPCR to quantify immunoprecipitated chromatin. (D-G) Chromatin immunoprecipitation using anti-Egr1, anti-H3K27me3 and anti-H3K4me3 antibodies. Precipitated DNA was analyzed by gRT rtPCR for enrichment on the indicated promoter. 
A

HOX cluster genes

Shcon ITS

$A 1$

B2
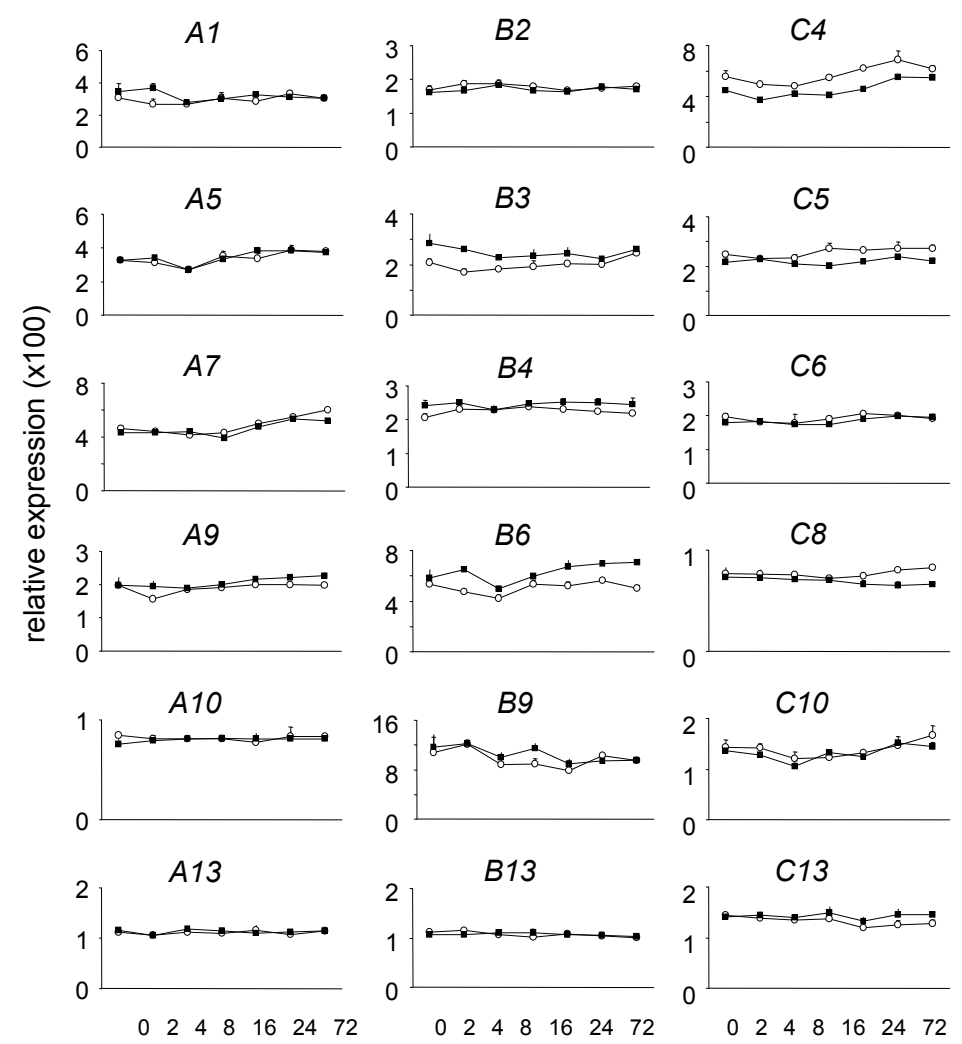

B3

C5
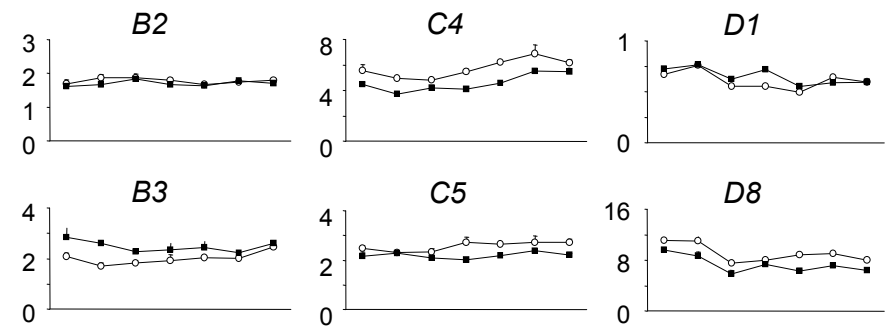

16. $D 8$
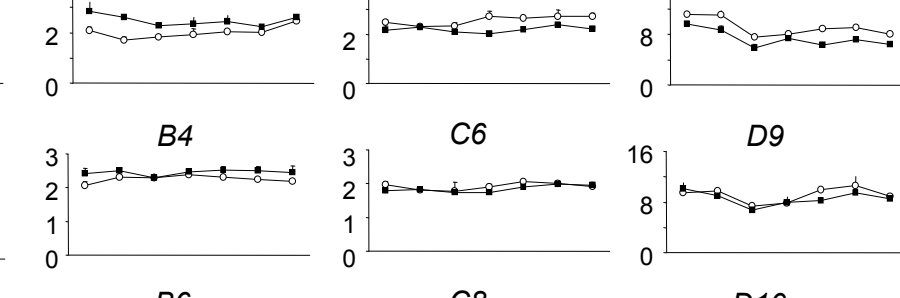

0

16 D9
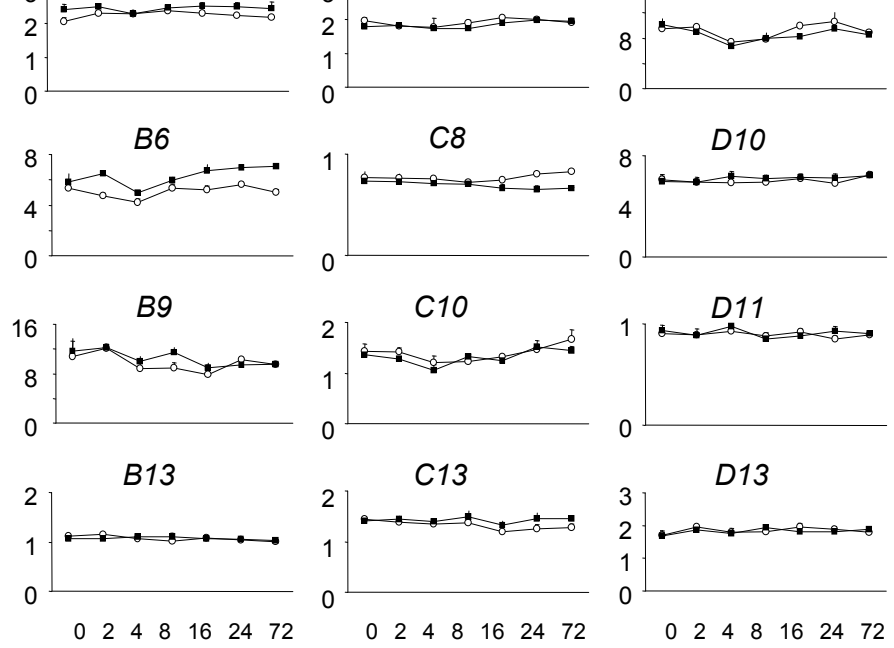

time pid (hours)

\section{B}

$\begin{array}{lcccc}\text { Cluster: } & \text { HOXA } & \text { HOXB } & \text { HOXC } & \text { HOXD } \\ \text { genes }-\mathrm{n}^{\#:} & \text { a4 }-3 & \text { b3 }-2 & \text { c11-1 } & \text { d1 }-1 \\ & \text { a10-1 } & \text { b4 }-2 & & \text { d3 }-1 \\ & & \text { b7 }-4 & & \text { d4 }-1 \\ & & \text { b8 }-1 & & \text { d8 }-2\end{array}$

Figure S9. Egr1 promoter binding is not sufficient for transcriptional activation.

(A) Microarray mRNA expression analysis of all Hox clusters in ATDC5 cells stably expressing shControl or shEgr1 throughout chondrogenesis. (B) The number of predicted Egr1 binding sites (GENOMATIX) in the promoters of the respective Hox genes. 
Chapter 4

Supplemental Table 1:

Primers used for qRT rtPCR

$\begin{array}{lll}\text { Ezh2 } & \text { Forward } & \text { GAAAAAAGATGAGACGTCCAGCTC } \\ \text { Aggrecan } & \text { Reverse } & \text { GAGGTTCAATATTTGGCTTCATCTTTAT } \\ & \text { Forward } & \text { CATGAGAGAGGCGAATGGAA } \\ \text { Sox9 } & \text { Reverse } & \text { TGATCTCGTAGCGATCTTTCTTCT } \\ & \text { Forward } & \text { AGTACCCGCACCTGCACAAC } \\ \text { Bmi1 } & \text { Reverse } & \text { TACTTGTAGTCCGGGTGGTCTTTC } \\ & \text { Forward } & \text { TGGAGAAGAATGGCCCACT } \\ \text { Collagen 2a } & \text { Forward } & \text { CAAGAACCCTGCTCGCACTT } \\ & \text { Reverse } & \text { CCGCTCTTCCACTCGGG } \\ \text { Egr1 } & \text { Forward } & \text { AGCCGAGCGAACAACCCTAT } \\ & \text { Reverse } & \text { TGTCAGAAAAGGACTCTGTGGTCA }\end{array}$

Primers used for Chromatin Immunoprecipitation

$\begin{array}{lll}\text { Sox9-1 } & \text { Forward } & \text { GTTCCAAAACTGTGACATTCCG } \\ \text { Sox9-2 } & \text { Reverse } & \text { TCCGCGTGTGTAAGTTTGCTCTCT } \\ & \text { Forward } & \text { TCTCCTGGACCCCTTCATGA } \\ \text { Sox6 } & \text { Foverse } & \text { GCCAGACAGGCCCTTCT } \\ & \text { Reverse } & \text { AAGCCAATTGTCCTTTGCAGTT } \\ \text { Hoxa1 } & \text { Forward } & \text { TCACTGAGTGATATTGGATCCTGC } \\ & \text { Reverse } & \text { GGAGGAAGTGAGAAAGTTGGCAC } \\ \text { Hoxa2 } & \text { Forward } & \text { GACAAGGTTGAAATTGGACCG } \\ & \text { Reverse } & \text { CAAATTGTCATTGGGCAGAAGC } \\ \text { Hoxa4 } & \text { Forward } & \text { CTCTGGAATAAAAGAAGGAGGC } \\ & \text { Reverse } & \text { GGACAAAGAATCAAAGGGCGAG } \\ \text { Hoxa6 } & \text { Forward } & \text { CTTTCCTTTTTGCCTTCATGG } \\ & \text { Reverse } & \text { TTGTCAGGTTTCCTGTTTGGG } \\ \text { Hoxa7 } & \text { Forward } & \text { AACCCTTCCCCTAAACGCCTC } \\ & \text { Reverse } & \text { AAAAGGTCGCCAGTCTTCCAG } \\ \text { Hoxa9 } & \text { Forward } & \text { ATCTGTATGCCTAGTCCCGCTCC } \\ & \text { Reverse } & \text { TTGATGTTGACTGGCGATTTTC } \\ \text { Hoxa11 } & \text { Forward } & \text { GGAAGCAACAGATCGTCACTCG } \\ & \text { Reverse } & \text { TGAGTTACACCGGCGATTACG } \\ \text { Ezh2 } & \text { Forward } & \text { GAGGCGCTTGATAGTGCTGG } \\ & \text { Reverse } & \text { ACCCAATCGCCATCGCT } \\ \text { Bmi1 } & \text { Forward } & \text { CATTTTGGAGCCGGTGTCA } \\ & \text { Reverse } & \text { AAAAAATGCACGGCTGAAGG }\end{array}$




\section{Chapter 5}

\section{Polycomb Repressive Complex 1 \\ Coordinates Enhanced Replication and Transcription in Transit Amplifying Chondrogenic Progenitors.}

Spaapen F, Eijssen L, Welting T, Salvaing J, Prickaerts P, Dahlmans V, Surtel DAM, Koseki H, Vidal M, Takihara Y, Wouters B, Voncken JW.

In preparation. 


\begin{abstract}
Segmentation phenotypes in Polycomb loss-of-function mutants correlate with defective maintenance of HOX gene expression boundaries along the antero-posterior axis, yet little is known about the possible direct involvement of Polycomb Repressive Complex 1 proteins (PRC1) in enchondral ossification. We here investigate chondrogenesis in relation to loss of Polycomb function. We provide evidence that normal chondrogenic hypertrophy in vitro and in vivo shares characteristics with replicative senescence. We find that acute shRNA-mediated reduction of PRC1-function compromises transit amplification during differentiation: rapid accumulation of massive DNA damage and activation of an intra-S-phase checkpoint result in a senescenceassociated secretory phenotype and a senescence-like replicative arrest. Using global nuclear analysis of active transcription and replication, we show that coordinated regulation of active transcription and replication is lost in Bmi1-deficient cells and that DNA damage occurs at sites of DNA synthesis. Hence, acute loss of PRC1-function during early replication in chondrogenesis renders cells unable to deal with increased chromatin stress. This is the first report revealing an important function for PRC1 in orchestrating simultaneous chromatin-associated processes, i.e. DNA replication and transcription damage repair, in differentiating transit amplifying cells.
\end{abstract}


Polycomb Group proteins act in transcriptionally repressive complexes (Polycomb Repressive Complexes; PRC), which vary in protein composition between cell types ${ }^{1}$. These multi-component PRCs maintain gene expression profiles in the context of differentiation and development. PRCmediated silencing is controlled at the chromatin level by epigenomic indexing: PRC complexes install and bind post-translational modifications on $\mathrm{N}$-terminal histone tails, often in conjunction with associated epigenetic activity ${ }^{2,3}$. Well-known PRC-associated epigenetic marks are H3K27me3 and H2AK119ub1, both are required for PRC-mediated transcriptional repression ${ }^{2-5}$. As such PRC are believed to constitute a molecular epigenetic memory and fulfill an important task in maintaining cell identity.

Analogous to PRC mutations in Drosophila Melanogaster, which cause abnormal segment identity development along the antero-posterior (AP) axis, the effect of PRC1 loss-of-function (LOF) on murine skeletal development correlates with defective maintenance of expression boundaries in HOX gene clusters ${ }^{6-9}$. The exact role of PRC1 in bone and cartilage formation however, is not clear. Moreover, the relatively mild skeletal phenotypes of numerous single PRC1-null mutants as opposed to compound PRC1 mutants ${ }^{9-13}$, suggest that redundant mechanisms may mask acute effects of loss-of-PRC1 function during skeletogenesis. The mature ossified mammalian skeleton develops from a cartilaginous scaffold (endochondral bone formation); we therefore studied chondrogenesis in vitro in the context of PRC1 loss-of-function (LOF). The murine ATDC5 embryonal mesenchymal stem cell line reiterates the multi-step chondrogenic differentiation program observed during endochondral bone formation and is an excellent, widely used in vitro model system to study chondrogenesis ${ }^{10}$ : a growth factor-induced differentiation program is initiated which is readily monitored by chondrogenic marker gene expression (e.g. SOX9, RUNX2, Collagen type II and X (COL2A1, COL10A1), Aggrecan (ACAN); ${ }^{11}$. We focused on PRC1 complex genes with known null-mutant skeletal phenotypes ${ }^{7,12-14}$. Several PRC1 complex genes are highly expressed, among which BMI1 (PCGF4), PHC1 and 2 (EDR1/MPH1/RAE28 and EDR2/MPH2) and $R N F 2$ (RING1B), and regulated throughout chondrogenesis (figure $S 1 \mathrm{~A}$ ): Bmi1 protein expression is elevated at 6 days (figure $1 \mathrm{~A}, \mathrm{~B}$ ) post induction of differentiation (pid), pointing to an increased reliance on Bmi1 function. Retroviral short hairpin RNAs (shRNA) were designed to target both human and mouse (shBM/hm) or exclusively human (shBM/h) BMI1 mRNA for RNAimediated degradation ((figure 1B,C; S1B). RNAi-mediated Bmi1 knock-down (KD) delays chondrogenesis as evidenced by reduced ACAN and COL2A1 expression (figure 1D); conversely shBM/h has no effect on differentiation, in line with its selective targeting of human BMI1 mRNA (figure S1C). In keeping with published gene dosage effects on skeletal phenotypes in PRC1 genetically modified mice ${ }^{6,8,12,13}$, re-expression of $m B M / 1$ cDNA in ATDC5-shBM/hm cells restores Bmi1 expression and induces the exact opposite effect of shBM/hm on chondrogenic marker gene expression (figure 1E, 1SD), Thus this data demonstrates the significance of the ATDC5 model for probing PRC1-function, and is consistent with a specific function for PRC1 in chondrogenesis. 
A

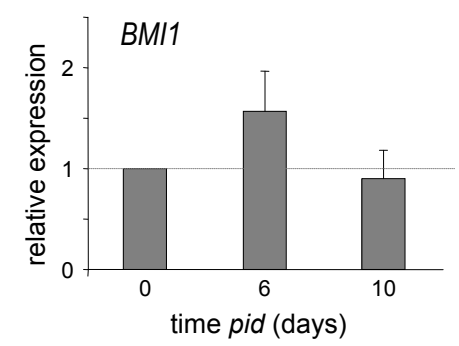

B

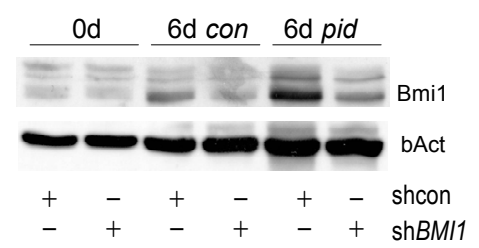

C

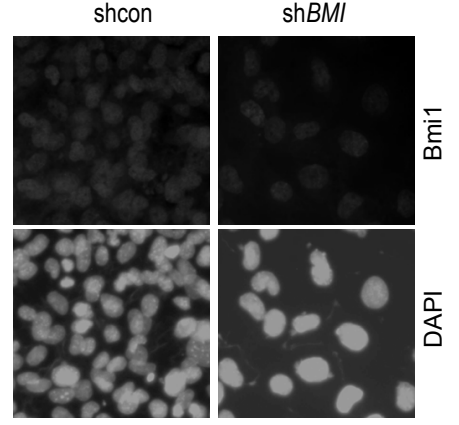

$\mathrm{t}=6$ pid (days)
D
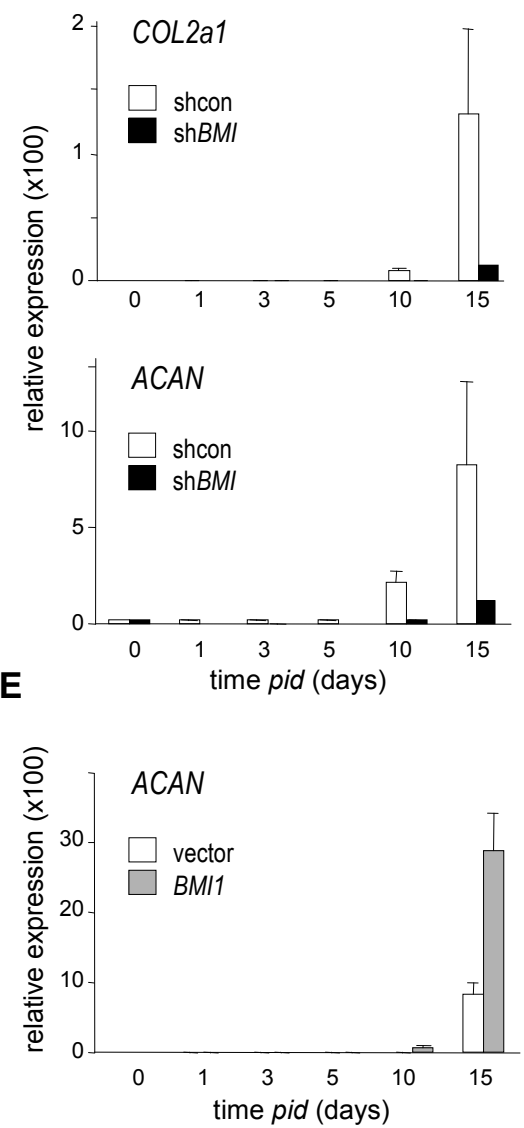

Figure 1. Bmi1 expression is required for chondrogenic differentiation.

(A) qRT rtPCR analysis of Bmi1 expression during ATDC5 chondrogenesis. (B) Western blot analysis of Bmi1 protein expression in ATDC5 cells stably expressing shControl or shBmi1 (control versus differentiation medium) at $\mathrm{t}=0$ and 6 days. (C) Immunofluorescence analysis of Bmi1 expression in ATDC5 shControl and shBmi1 and DAPI nuclear staining. (D) qRT rtPCR analysis of chondrogenic markers in ATDC5 shControl and shBmi1 up to 15 days pid. (E) qRT rtPCR analysis of ACAN in Bmi1 overexpressing ATDC5 and control cells up to 15 days pid.

Numerous published data support a role for PRC1 proteins, including Bmi1, in cell cycle regulation under normal and oncogenic conditions ${ }^{17-20}$. The exact mechanism(s) by which PRC control cell cycle in different settings, however, are insufficiently clear. Differentiating ATDC5 monolayer cultures typically become multi-layered and develop distinctive three-dimensional nodules that produce cartilage matrix proteins ${ }^{10}$. In line with a reduced chondrogenic potential, noduleformation is delayed and reduced in numbers in Bmi1-KD cultures (see below: figure 4C). As multilayering and nodule formation are, at least in part, dependent on sustained proliferation, we examined cell proliferation in relation to PRC1-LOF. Under non-differentiating conditions, Bmi1-KD marginally affects cell proliferation (figure $2 A, B$ ). Upon induction of differentiation, proliferation rate in control cultures increases substantially from 1 day pid (fig $2 A$ ) and is sustained until 5-6 days 
PRC1 Coordinates Replication and Transcription in Chondrogenic Progenitors pid; this early, rapid, transient increase in cell division, is observed in various cell systems and resembles transit amplification (TA) of progenitor cells ${ }^{15,16}$. Relevantly, transient rapid progenitor proliferation is part of normal endochondral ossification in vivo, and is apparent in the proliferative zone of growing long bones (fig S2A). Remarkably, Bmi1-KD cells fail to undergo transit amplification pid: cell proliferation does not exceed that of cells (Bmi1-KD or control) under continuous culture conditions (figure $2 \mathrm{~B}$ ). Of note: the proliferative burst of differentiating ATDC5 cells corresponds in time with elevated Bmi1 expression (figure 1A,B), suggesting transit amplification is either controlled by or controlling Bmi1 levels. Transit amplification responds positively to Bmi1 dosage: overexpression of Bmi1 increases proliferation slightly yet significantly (figure S2A). Under non-differentiating conditions, DNA profiling and BrdU incorporation assays reveal neither significant differences in cell cycle phase distribution nor in BrdU incorporation (figure 2C, S2B) between Bmi1-KD and control cells. In sharp contrast, differentiating Bmi1-KD cells show a substantially increased S-phase population at 6 days pid (figure S2B). Similarly Phc2KD reduces cell proliferation pid and increases S-phase accumulation, pointing to a general involvement of PRC1 in transit amplification (figure S2C). Transient BrdU exposure at 6 days pid reveals active incorporation of BrdU by rapidly dividing control cells and confirmed a block in de novo DNA synthesis in Bmi1-KD cultures (figure 2C). Consistent with this, differentiating ATDC5 Bmi1-KD cultures display significantly less G2/M related H3S10 phosphorylation (figure 2D). Importantly, normal healthy human cartilage-derived chondrogenic precursors respond similarly to BMI1-LOF: proliferative and chondrogenic capacity are severely reduced (figure 2E). Combined, the above data support the notion that cells lacking PRC1 cannot replicate their DNA as efficiently as control cells during the hyperproliferative phase. An intra-S-phase arrest is indicative of stalled replication forks; these are known to coincide with double strand DNA breaks (DSB) and locally induce Ser139 phosphorylation on histone $\mathrm{H} 2 \mathrm{AX}(\gamma \mathrm{H} 2 \mathrm{~A} . \mathrm{X})$. Under normal (non-differentiation) culturing conditions, ATDC5 cultures (independent of genotype) typically include some cells carrying $\gamma \mathrm{H} 2 \mathrm{~A}$.X foci, presumably due to local replication-associated DSB (figure S3A,B). Normally such DNA damage is rapidly repaired to ensure cell cycle completion ${ }^{17}$. At 6 days pid, however, $\gamma \mathrm{H} 2 \mathrm{~A}$.X detection in Bmi1-KD cultures is dramatically increased compared to control cells (figure 3A). Differences in DSB are first detectable at 1-2 days pid (figure 3B); at 10 days pid DNA damage is substantially reduced (figure $3 \mathrm{C}$ ); control cultures do not accumulate as much damage, yet show detectable $\gamma \mathrm{H} 2 \mathrm{~A} . \mathrm{X}$ throughout the proliferative phase (figure $3 \mathrm{C}$ ). Differentiating Bmi1deficient primary human h929 cells also show elevated $\gamma \mathrm{H} 2 \mathrm{~A} . \mathrm{X}$ (figure 3D). High-density culturing of chondrogenic models triggers differentiation even in the absence of ITS. Consistent with differentiation-induced DSB, high-density Bmi1-KD cultures show S-phase accumulation and increased DNA damage as opposed to control cultures (Figure S3C). Whereas Phc2-LOF has comparable effects on progenitor expansion under these conditions (figure S3D), interestingly, the effect of PRC1-LOF on chondrogenic potential differs between PRC1 members (figure S3E). 
A
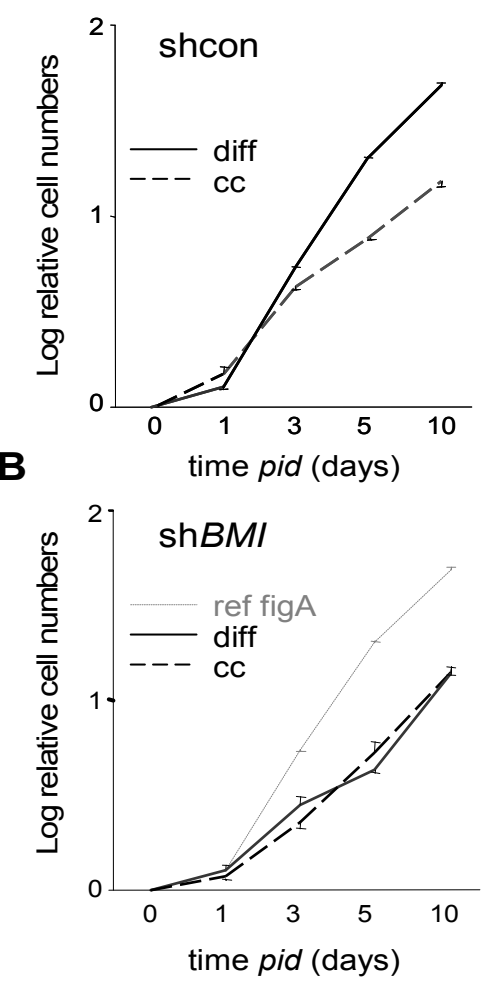

$\mathbf{E}$

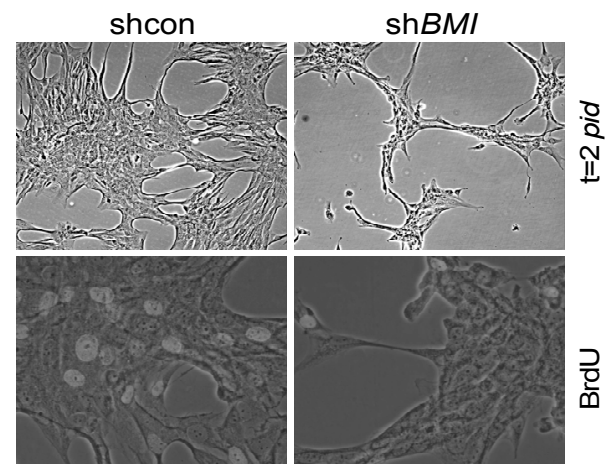

C

D
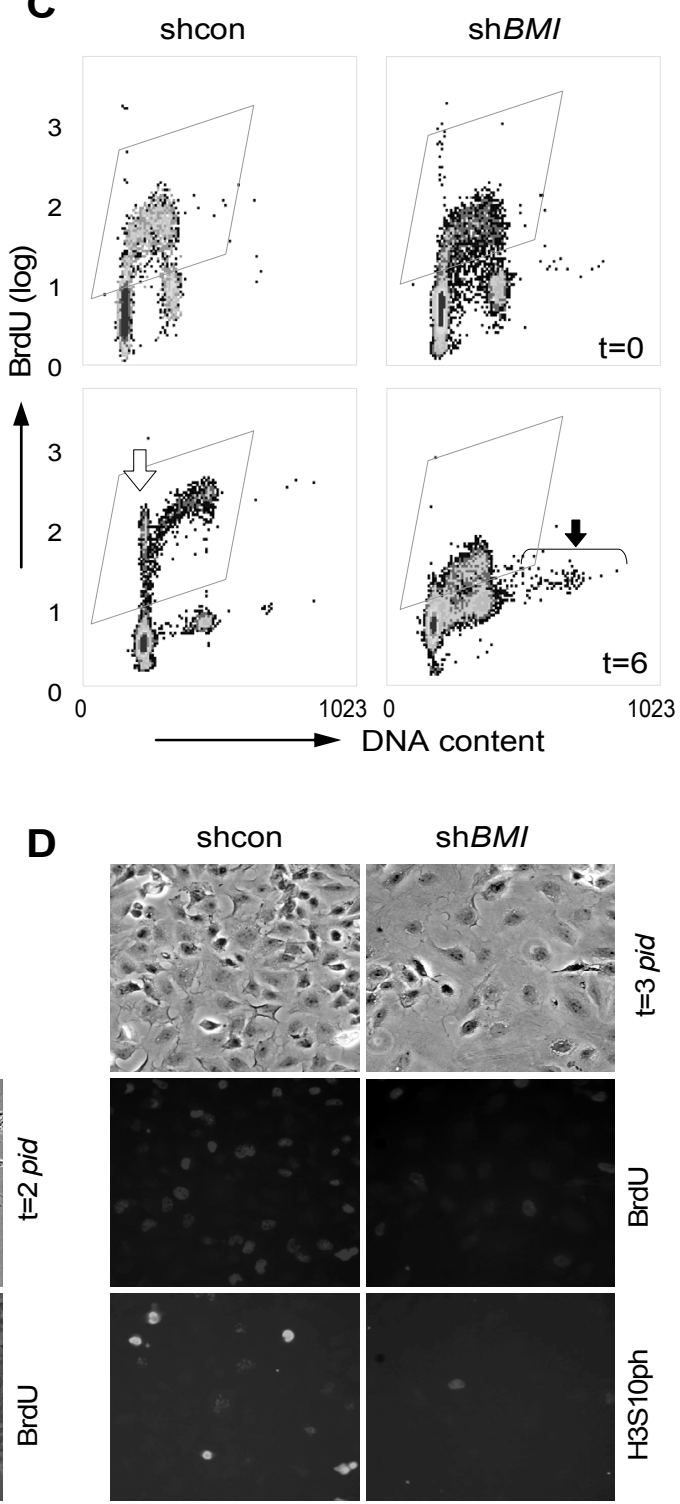

Figure 2. Loss of Bmi1 elicits replication stress induced S-phase arrest.

(A) ATDC5 cells hyperproliferate upon induction of chondrogenic differentiation, as compared to continuous culture conditions (cc). Proliferation determined by Crystal violet incorporation with subsequent extraction and spectrophotometric analysis. (B) ATDC5 cells stably expressing shBmi1 fail to undergo differentiation induced hyperproliferation, measured by Crystal violet incorporation. (C) ATDC5 cells stably expressing shEgr1 or shControl were differentiated for 6 days, then BrdU was administered to the cells for 45 minutes. BrdU was visualized with anti-BrdU antibodies and subsequently analyzed by flowcytometry. DNA content is determined by propidium iodide staining. (D) ATDC 5 shBmi1 or shControl were differentiated for 3 days, then BrdU was administered to the cells for 45 minutes. BrdU was visualized by immunofluorescence with anti-BrdU antibodies. (E) Human primary chondrogenic precursors stably expressing shBmi 1 or shControl were differentiated for 2 days, then BrdU was administered to the cells for 45 minutes. BrdU was visualized by immunofluorescence with anti-BrdU antibodies.

This phenotypic variation may reflect divergent effects of PRC1-LOF on skeletal morphogenesis in full genetic knock-outs in vivo ${ }^{6,14}$. In line with a proliferative block, detection of nuclear $\gamma \mathrm{H} 2 \mathrm{~A}$.X and H3S10ph inversely correlate between control and KD cells at 3 days pid (figure S4A). Expression array analysis at 6 days pid confirms massive upregulation of DNA damage repair pathways in Bmi1-KD cultures (Table S1); consistent with activation of a DNA damage response (DDR) Bmi1KD nuclei show ATM-induced Kap1S824 and Chk2-phosphorylation besides enhanced Rad51 and RPA70 chromatin-association (Figure S4B-D; and data not shown). 
A

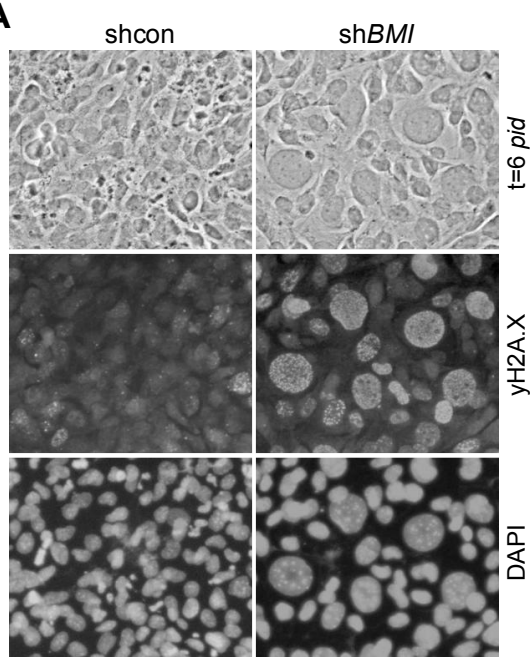

C

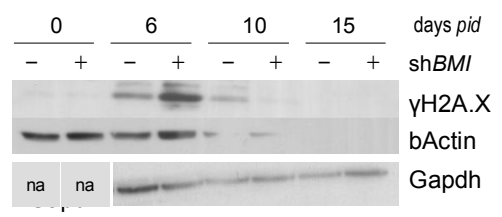

B

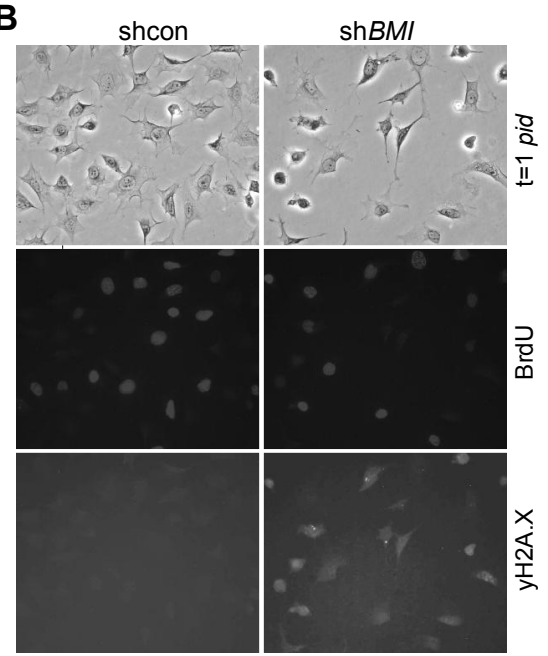

D

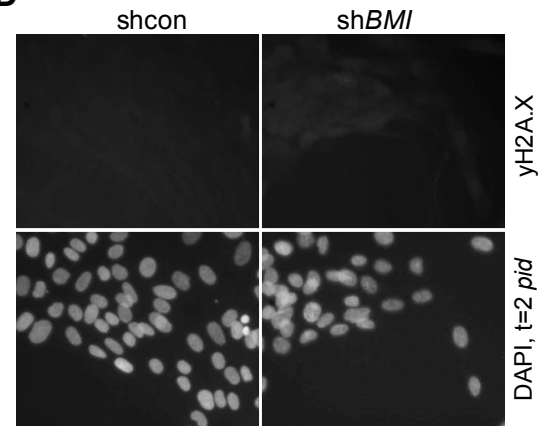

Figure 3. Replicating shBmi1 expression ATDC5 cells accumulate DNA damage during differentiation induced transit amplification.

(A) Immunofluorescence analysis of DNA damage in ATDC5 shControl and shBmi1 visualized with antiYH2A.X antibodies at 6 days pid. DAPI used as nuclear staining. (B) Immunofluorescence analysis of DNA damage and replication in ATDC5 shControl and shBmi1 visualized with anti-yH2A.X and anti-BrdU antibodies, respectively, at 1 day pid. DAPI used as nuclear staining. (C) Western blot analysis of $y H 2 A . X$ in ATDC5 shControl and shBmi1 throughout differentiation. B-actin and Gapdh as loading controls. (D) Immunofluorescence analysis of DNA damage in human primary chondrogenic progenitors expressing shControl and shBmi1 visualized with anti- $\mathrm{H} 2 \mathrm{~A} . \mathrm{X}$ antibodies at 2 days pid. DAPI used as nuclear staining.

Loss of PRC1-function causes premature replicative senescence in mouse embryo-fibroblasts cultures and other cell types ${ }^{18-20}$. Analogously, Bmi1-deficiency induces a cell senescence-like morphology as does LOF of other PRC1-proteins (figure 4A). The presence of $\gamma \mathrm{H} 2 \mathrm{~A}$.X in enlarged cells at 6 days pid suggests that these cells probably accrued DSB and activated a DDR and an associated senescence-like response (SLR) overtime (figure $3 A$ ), in agreement with the causal relationship between DNA damage and senescence ${ }^{21,22}$. In line with this notion, also sporadic large flat cells in non-differentiating cultures carry DNA damage and are polyploid irrespective of genotype; large flat Bmi1-KD cells again show substantially more DSB (figure S5A). The relative resistance of ATDC5 under normal growth conditions to Bmi1-LOF was unexpected given its otherwise significant anti-proliferative effect in cell cultures ${ }^{26,27,23}$. This prompted us to investigate 
senescence-related signaling in the context of differentiation. Of note: important checkpoints (i.a. contact inhibition, IR induced arrest/senescence; data not shown) are intact in non-differentiating ATDC5. Senescent fibroblasts typically express senescence-associated ß-Galactosidase (SAbGal/Glb1) ${ }^{20}$. Surprisingly, GLB1 mRNA is induced approximately 2 fold and SAbGal is activated during chondrogenesis in vitro (figure 4C, S5B). Importantly, we observe SAbGalactivation in all primary human and rabbit chondrogenic cultures examined (figure S5C). Rapidly expanding murine ATDC5 and primary human h929 cells display focal H3K9me3, a feature reminiscent of senescence associated heterochromatin foci (SAHF; ${ }^{29}$ ) and Bmi1-LOF further augments H3K9me3 levels (figure 4B,D, S5C). Besides a global increase of H3K9me3, H3K27me3 increases during hyperproliferative stress in Bmi1-KD cultures (figure 4C,E). Ezh2 protein is slightly more expressed in Bmi1-KD cells at 6 days pid and may contribute to enhanced H3K27me3 levels (figure S5E). However, the H3K27me3 demethylase Jmjd3, which is normally induced 4.2 fold during hyperproliferation, fails to do so in Bmi1-KD 6 days pid (figure S5F). Lack of JMJD3 was recently associated with replicative senescence ${ }^{24}$; hence it is likely that absence of Jmjd3 is responsible for the senescence-associated increase in H3K27me3. In good agreement with activation of SLR, numerous additional markers associated with cell cycle exit and/or a senescence-associated secretory phenotype $\left(\mathrm{SASP} ;{ }^{25}\right)$ are subject to dynamic regulation in the context of normal differentiation and show an exaggerated response (i.a. IL6, CCL5, CXCL10) in Bmi1-LOF cultures during hyperproliferation (figure S5G). Bmi1-LOF does not appear to change the onset of SAbGal activation (figure 4C). Remarkably, although overexpression of oncogenic RasV12 in chondrogenic control cultures enhances onset and intensity of SAbGal activation and, it does not induce replicative arrest and leaves chondrogenic potential fully intact (figure 4C, S5H).

We next explored involvement of known senescence-related checkpoints. PRC1-dependent transcriptional regulation of the $C D K N 2 A / I n k 4 A$ locus ${ }^{26}$ links Bmi1 directly to cell cycle regulation, stem cell self-renewal and oncogenesis ${ }^{27-33}$. We could not detect significant differences in

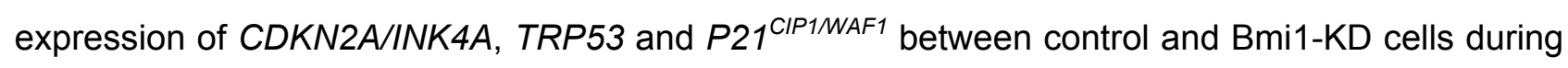
chondrogenesis (figure $\mathrm{S} 5 \mathrm{C}$ ). In addition, preliminary analyses indicate that inactivation of INK4A/ARF or TRP53 does not bypass cell cycle arrest in ATDC5 Bmi1-KD pid cells, but in stead reveals a dependency of chondrogenesis on these cell cycle regulators (FS, JWV unpublished observations). These findings are in agreement with published reports showing that the intra-Scheckpoint, although pRb and/or Trp53-dependent, is not induced by $\mathrm{p} 16^{\text {INK4A }}$ or P2 $1^{\text {CIP1/NAF } 3834}$. In support of this, a differentiation and S-phase arrest by CHAF1A/CAF1b-LOF in zebrafish was not rescued by Trp53-LOF ${ }^{35}$. Our observation in ATDC5 contrasts with a recently uncovered lineagespecific role of $\mathrm{p} 21^{\mathrm{CIP} 1 / \mathrm{WAF} 1}$ in NSC self-renewal ${ }^{36}$, and suggests lineage specific PRC-independent checkpoint mechanisms are operative during self-renewal and/or transit amplification in chondrogenic. Taken together, above observations suggest that the chondrogenic lineage is inherently refractory to signaling known to induce irreversible replicative arrest in other systems. 
A

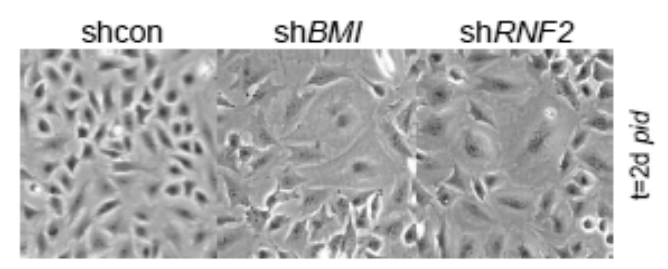

B

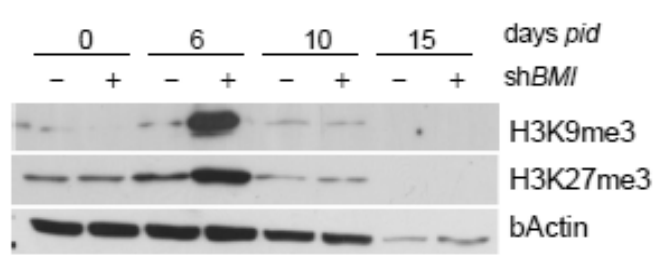

D

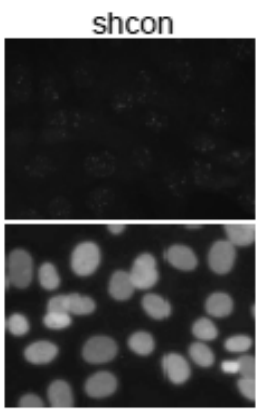

E

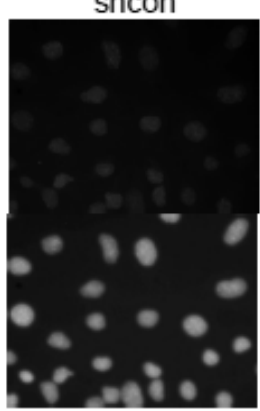

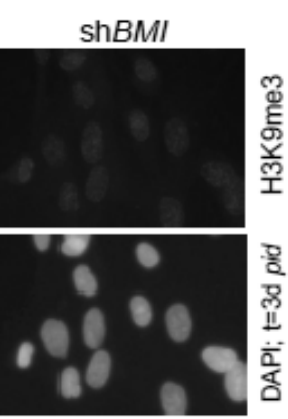

shBMI

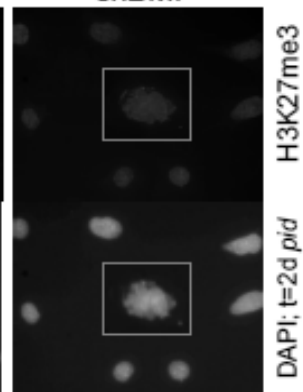

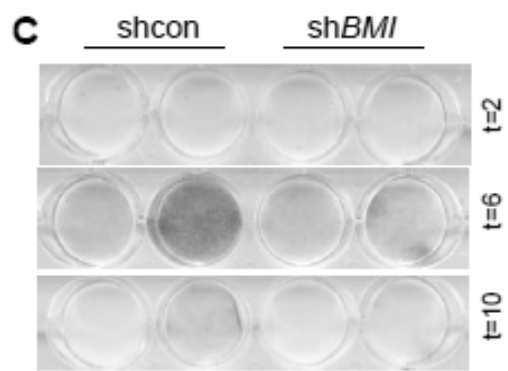

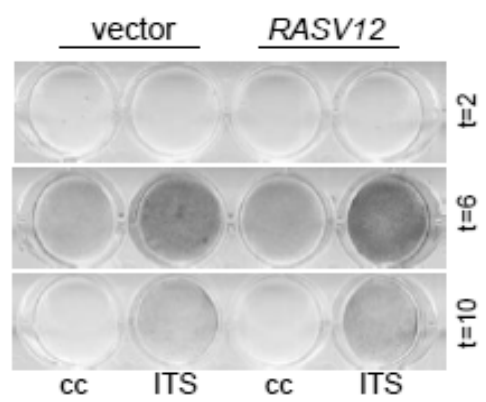

$\mathbf{F}$

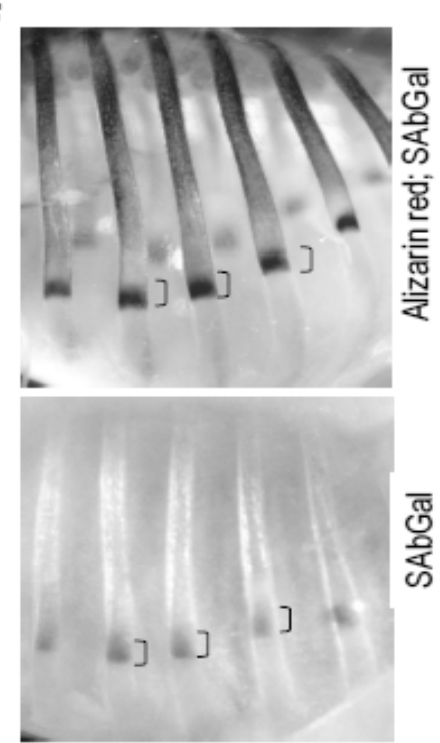

Figure 4. PRC1-LOF results in replicative senescence; terminal chondrogenic differentiation resembles senescence.

(A) Morphological analysis ATDC5 shControl, shBmi1, shRnf2 at $t=2 d$ pid. (B) Western blot analysis of senescence associated histone modifications in ATDC5 shControl and shBmi1 throughout chondrogenesis. (C) Senescenceassociated bGalactosidase staining on ATDC5 expressing shControl, shBmi1, vector or Rasv12 cultures under control conditions or differentiation conditions (ITS) for the indicated time points. (D,E) Immunofluorescence analysis of senescence associated histone modifications in ATDC5 shControl and shBmi1 at indicated time points. DAPI used as nuclear staining. (F) Senescence-associated bGalactosidase staining only or in combination with alizarin red on ribcages of WT mice. Brackets highlight the hypertrophic zone.

Chondrogenic differentiation during endochondral ossification terminates in hypertrophy and subsequent tissue replacement (mineralization/ossification) by invading osteoclasts and osteoblasts ${ }^{11}$. Importantly, RUNX2, an oncogene known to activate SLR, is implicated in hypertrophic differentiation ${ }^{37,11}$. Based on our findings thusfar, we hypothesized that endochondral differentiation may naturally terminate in a senescence-like phenotype, and thus SAbGal-positive cells should be detectable adjacent to growth plates in elongating bones of newborn mice. In line with activation of SAbGal during chondrogenic hyperproliferation in vitro, histological analysis of pp1 (day 1 post-partum) skeletal elements revealed prominent SAbGAl activity precisely at the prehypertrophic zone in long bones and intercalated disks (figure 4F, S6A,B). SAbGal positive 
zones are positioned proximal to mineralization zones in rib bones (figure 4F). We reasoned that PRC1-LOF may result in increased hypertrophy during endochondral ossification which should be reflected in the width of the $\mathrm{HZ}$. In good agreement with this, RING1A-KO pp1 mice, which display a comparatively prominent PRC1-LOF related skeletal phenotype ${ }^{14}$, revealed an increased $\mathrm{HZ}$ (Figure S6C). Thus, our collective findings corroborate a hitherto unrecognized role for PRC1 in chondrogenic transit proliferative amplification.

\section{A}

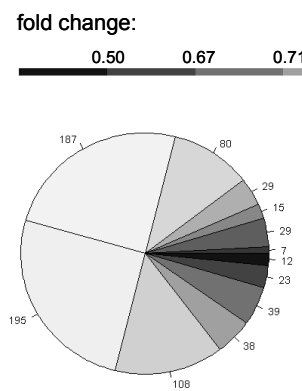

$\mathrm{t}=0 ; 12.7 \%[3.7 \%]$

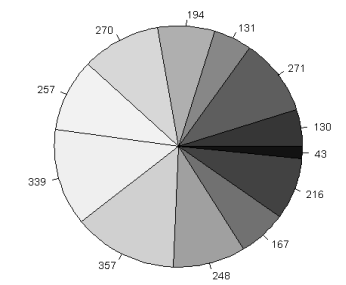

$\mathrm{t}=1 \mathrm{~d}$ pid; $43.4 \%[11.0 \%]$

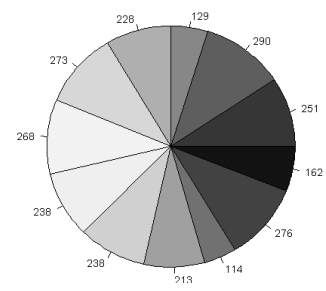

$\mathrm{t}=6 \mathrm{~d}$ pid; $43.3 \%[11.3 \%]$

B

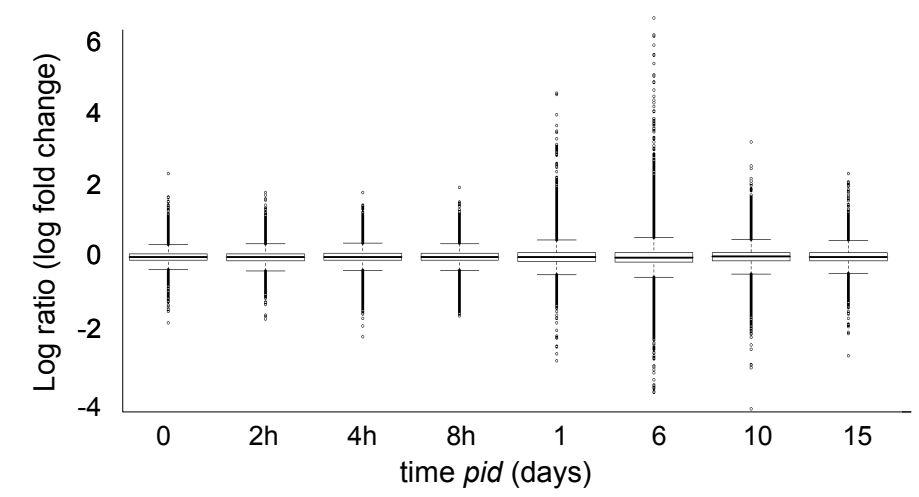

C
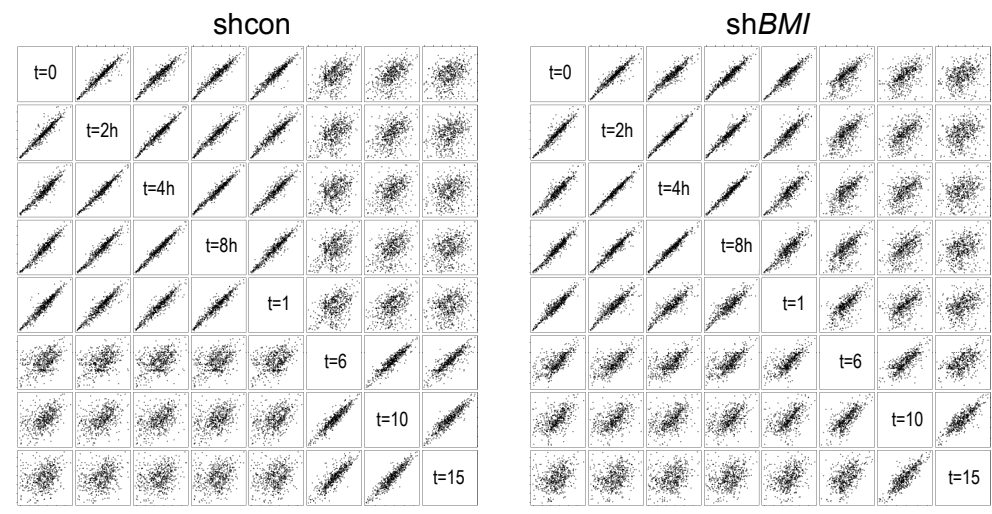

Figure 5. Fold change increases during transit amplification.

(A) Pie charts of all reporters fulfilling $p<0.05$, FC $>1.1$ up or down, expression $>{ }^{2} \log (100)$ in either group for time points 0,1 day and 6 days pid respectively (for all time points c.f. Figure S7A). For each time point two percentages are indicated: the first indicates the amount of reporters fulfilling all criteria as compared to the number of reporters above expression limit; the second indicates the amount of reporters fulfilling all criteria as compared to the total number of reporters. (B) Boxplots of WT vs. Bmi1-KD logratios for all reporters (regardless of expression level, fold change or significance) for each time point in the experiment. (C) Scatterplot for each timepoint versus each other timepoint of the ${ }^{2}$ log expression values in WT (left) and Bmi1-KD (right) cells, based on the reporters fulfilling $p<0.05$, absolute FC $>2$, expression $>{ }^{2} \log (100)$ in either group, at 1 or 6 days pid. 
We next investigated the origin of DNA damage. As differentiation requires coordinated regulation of several DNA-templated processes, among which transcription and replication, we analyzed the effect of Bmi1-LOF on global transcriptional responses in the context of differentiation. Contrary to expectation, loss of repressive function produced comparable percentages up or down regulated genes (figure 5A, S7A). Transcriptional re-profiling (i.e. number of genes involved and fold changes) intensifies when transit amplification is initiated both in control and Bmi1-LOF cultures; however, differences in log expression prior to and following transit amplification are substantially smaller in control cultures that in Bmi1-LOF cultures, indicating much tighter controlled transcriptome reprogramming in the presence of Bmi1 (figure $5 \mathrm{C}$ ). Differences in gene expression between control and Bmi-LOF cultures become more prominent between 1 and 6 days pid: expression of over one third of genes is more than 1.2 fold enhanced (figure 5B, S7B). Transcriptomic deregulation revealed distinctive clustering (figure $5 \mathrm{C}, \mathrm{S7C}$ ). Profile analysis of the most extreme deregulated genes (i.e. $\geq 2$ fold changed) reveals a number of different profile clusters: genes that are consistently a) higher or b) lower expressed in Bmi1-KD versus control throughout differentiation, c) genes of which transcription is low and not regulated during chondrogenesis in control cells, but that dramatically respond to PRC1-LOF at the time when control cells enter the hyperproliferative state, and vice versa d) genes that are expressed and induced at hyperproliferation in control but fail to do so in Bmi1 KD cells (figure S7D). Numerous of the abnormally highly induced genes in KD cultures, map to differentiation programs and pathways irrelevant to chondrogenesis (figure S7E). A number of genes in category $c$ is related to SLR/SASP (figure S7F; see also figure S5C). In addition, induction of some genes appears inconsistent with chondrogenic differentiation. Per example, $\gamma$ SCrystalline (CryGS), a major functional component of the lens, is normally exclusively expressed by cell of ectodermal origin (ATDC5 cells are mesodermal derivatives). In line with an aberrant induction of CryGS during chondrogenesis, the crystalline gene cluster was recently identified as PcG target in cells of mesenchymal origin ${ }^{38}$. The diversity of aberrant response profiles combined with the observation that many genes are downregulated upon PRC1-LOF suggests that besides a function in (direct) target gene repression, PRC1 fulfills additional tasks that impinge on transcription. We therefore focused on the potential role of transcription and replication in the origin of DDR and consequential S-phase arrest. Typically, BrdU-positive cells co-stain for the DNA polymerase processivity factor PCNA (Proliferating Cell Nuclear Antigen) in control cultures (figure S8B); PCNA-dim cells are either in G1 or G2. Consistent with an intra-S-phase arrest, we find a large percentage of cells PCNA positive, yet BrdU negative (figure S8B). Importantly, all BrdU-positive cells are also positive for $\gamma \mathrm{H} 2 \mathrm{~A} . \mathrm{X}$ in Bmi1-KD cultures (figure 6A); cells that exclusively show $\gamma \mathrm{H} 2 \mathrm{~A}$.X signals arrested prior to BrdU incubation and, moreover, DNA damage in Bmi1-KD cells perfectly co-localizes with BrdU signals (figure 6A). This strongly supports the idea that damage occurs at sites of active de novo DNA synthesis and that lack of PRC1 is causally related to replication induced DNA damage. The 
Chapter 5

more intense staining for Rpa70 (figure S4A), a protein which prevents single stranded DNA from back-folding during DNA-replication, is in good agreement with replication-induced damage. Indeed, Bmi1-KD cultures are significantly more sensitive to the replication poisons etoposide than control cells under normal culturing conditions (figure S8A) (camptothecin similar results; data not shown).

To study transcriptional status, cells were stained for active Ser5-phosphorylated RNA polymerase 2 (pRpol2). Control cells reveal a cell cycle dependent non-uniform nuclear distribution of active pRpol2: pRpol2-bright cells are generally PCNA-dim (G1/early S), whereas late in S-phase pRpol2-negative nuclei are strongly positive for PCNA; likewise, these cells are DAPI-bright (figure $\mathrm{S8C}$ ); pRpol2-dim nuclear regions in control cells show BrdU incorporation (figure $6 \mathrm{~B}$ ). This nuclear partitioning of DNA-templated activities indicates coordinated regulation of replication and transcription under normal chondrogenic conditions and is in good agreement with earlier reports on dynamic segregation of mRNA and DNA synthesis throughout S-phase ${ }^{39}$. Remarkably, the non-uniform distribution of pRpol2 is lost in PCNA-bright PRC1 LOF cells, and, moreover, Bmi1-KD nuclei arrested in S-phase show uniform nuclear staining for both pRpol2 and BrdU (figure 6B; $\mathrm{S} 8 \mathrm{C}$ ), suggesting that concerted regulation of replication and transcription is lost in the absence of intact PRC1 (figure 6B). In addition, damaged Bmi1-KD cells have reduced pRpol2 levels, suggesting reduced transcription as a functional consequence of global DNA damage (figure 6C). DNA damage as a result of failure to tune replication to transcription, and vice versa, is expected to not be restricted to abnormally derepressed transcriptional activity, but instead occurs throughout the genome. The finding that many genes show consistent deregulated transcription, regardless of chondrogenic induction (figure 5C) most likely directly relates to defective transcriptional/replicational coordination and enhanced chromatin stress in PRC1-KD cultures.

Bmi1 and Rnf2, were recently identified as a ubiquitin E3 ligase for Geminin (Gmnn/Gem), a factor important for termination of replication at late S-phase ${ }^{40,41}$. PRC1-LOF stabilizes Gmnn and interferes with haematopoietic stem cell (HSC) self renewal ${ }^{41}$. In good agreement with this, we find that Bmi1-KD cells stain positive for Gmnn (Figure 7A), whereas proliferating control cells generally do not. In line with the observed DDR and SLR, all Gmnn positive Bmi1-KD cells co-stain for yH2A.X, and the Cdt1/Gmnn ratio $<1$ is indicative for re-replication and polyploidy in Bmi1-KD cells (figure 7B). The enhanced Gmnn chromatin-association shows an inverse correlation with global loss of Bmi1 (figure 1C). We recently observed a positive correlation between PRCphosphorylation, PRC1/chromatin dissociation and Gmnn stabilization (VvL JWV unpublished observations). In the context of stress signaling, redistribution of Phc1 (Rae28), through which Gmnn is associated with PRC1, may provide a docking point for Gmnn stabilization; stress signaling induces chromatin dissociation of other PRC1 members (Bmi1, Ring and CBX proteins; JBC \& YT, JWV; unpublished data). 
A

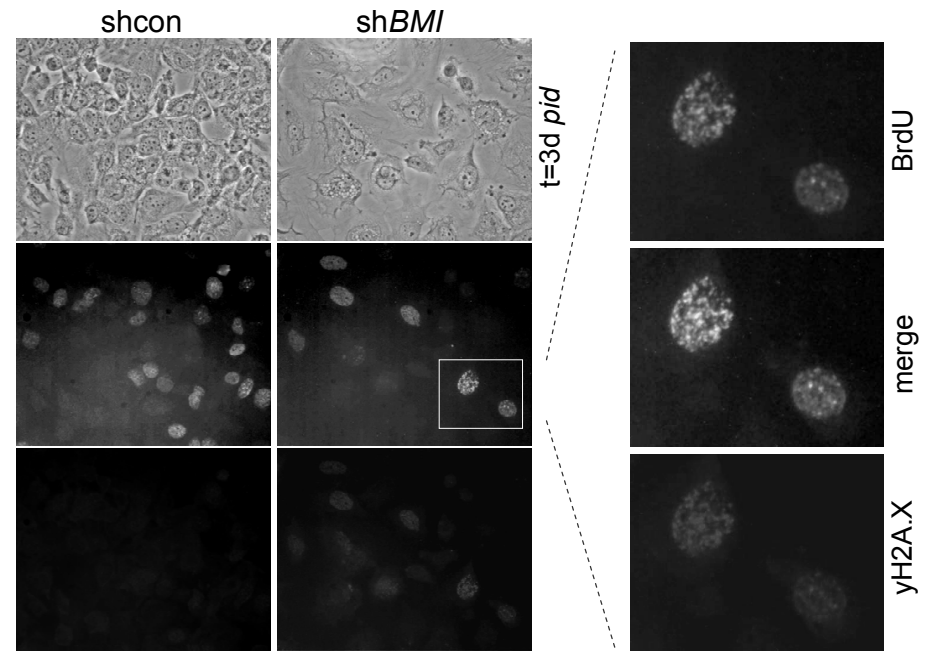

B

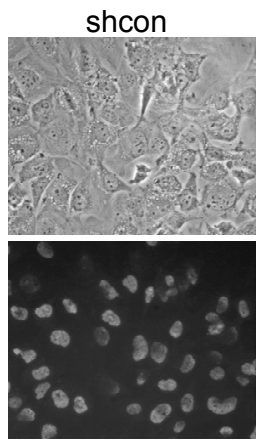

$\operatorname{sh} B M I$

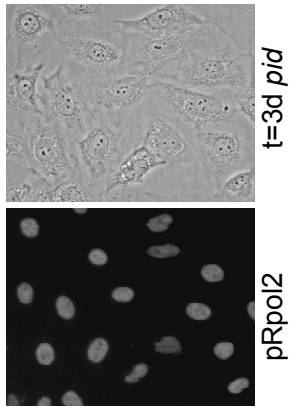

C
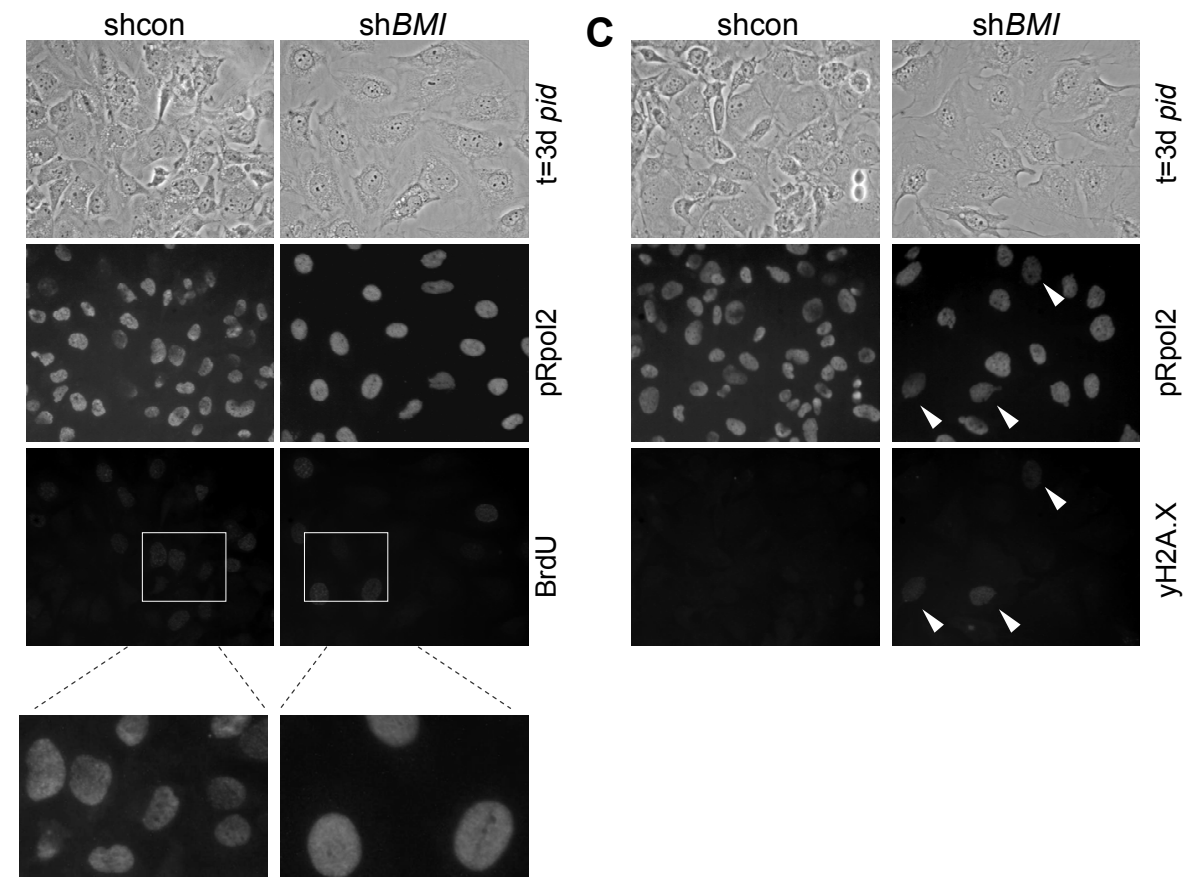

Figure 6. ATDC5 shBmi1 accumulate DNA damage at sites of deregulated transcription and replication.

$(A, B, C)$ Immunofluorescence analysis of replication (BrdU), active transcription (pRNA polymerase II) and DNA damage (YH2A.X) in ATDC5 stably expressing shControl or shBmi1 at 3 days pid.

Combining these observations, we propose a molecular switching mechanism that controls local transcription versus replication: local presence of Rnf2/Bmi1 represses transcription (H2AK119ub1), targets GMNN for proteolysis, relieving a potential replication block. Conversely, loss of BMI1/RNF2 stabilizes GMNN and blocks replication and is permissive for transcription (figure 7C).

The most direct interpretation of our data is that PRC1 generates a chromatin state which tunes transcription to DNA replication and prevents DNA-damage during a differentiation-coupled proliferative burst. Polycomb proteins are found at promoters also when genes are not silenced ${ }^{42}$. 
A
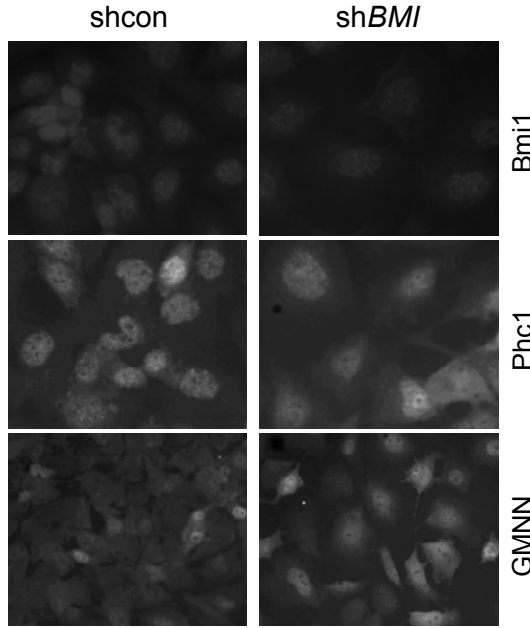

B
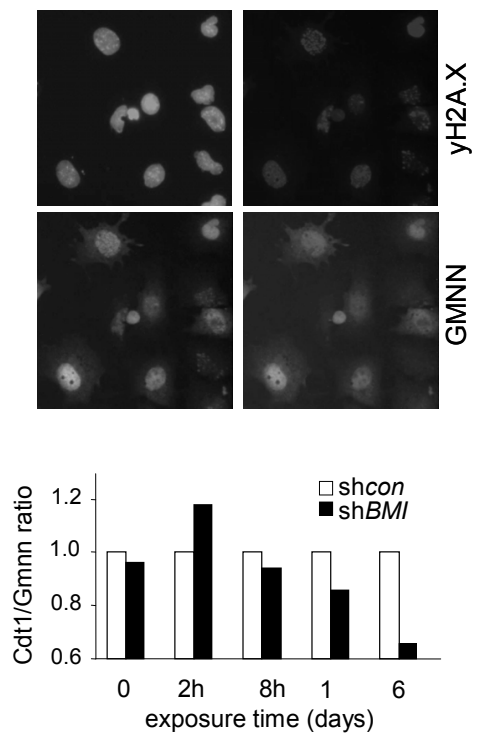

C

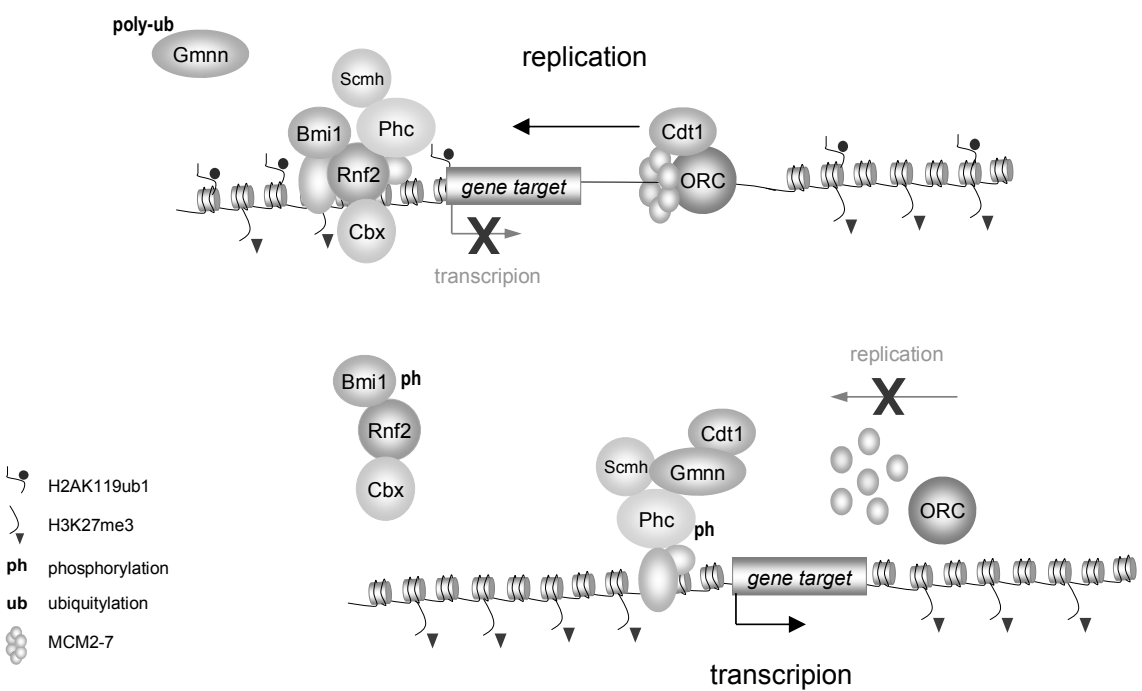

Figure 7. Accumulation of DNA damage correlates with stabilization of the replication inhibitor geminin.

(A) Immunofluorescence analysis of Bmi1, Phc1 and Geminin in ATDC5 shControl and shBmi1. (B) Immunofluorescence analysis of $\mathrm{yH} 2 \mathrm{~A} . \mathrm{X}$ and Geminin in ATDC5 shControl and shBmi1. Cdt1/Geminin ratio <1 is indicative for re-replication and polyploidy.

(C) Proposed model: PRC1 regulates a switching mechanism that controls local transcription versus replication: local presence of Rnf2/Bmi1 represses transcription (H2AK199ub1), targets geminin for degradation, relieving a potential replication block. Conversely, loss of Rnf2/Bmi1 stabilizes geminin and blocks replication and is permissive for transcription.

A substantial amount of H3K27me3 marked genes is expressed in ES cells; in addition, numerous gene promoters are bivalently marked (i.e. with both activation-associated $\mathrm{H} 3 \mathrm{~K} 4 \mathrm{me} 3$ and repression-associated $\mathrm{H} 3 \mathrm{~K} 27 \mathrm{me} 3 ;{ }^{43}$ ). Many of these promoters map to important developmental control genes, and bivalency often resolves into monovalency, dictated by their involved in specific lineage commitment programs. Gene expression and replication timing are strongly correlated: actively transcribed genes often replicate early. Both replication timing and transcription change substantially in the context of differentiation in mid S-phase, when replication origins are spatially redefined and replication timing and transcription are re-tuned ${ }^{44}$. Although a single study into 
PRC1 Coordinates Replication and Transcription in Chondrogenic Progenitors effects of PRC2-LOF on replication timing within the context of neurogenic differentiation revealed no obvious differences, a potential role for PRC1 in replication timing requires analysis in the context mesenchymal lineage commitment ${ }^{45}$. In addition to a direct involvement in transcriptional repression, it is conceivable that $\mathrm{H} 3 \mathrm{~K} 27 \mathrm{me} 3$ and $\mathrm{H} 3 \mathrm{~K} 4 \mathrm{me} 3$ (respectively PcG and trxG-associated epigenetic marks) functionally partition the genome and help coordinate DNA-templated processes like transcription and replication in the context of development. Indeed, PRC proteins interact with basal factors of the transcription machinery as well as replication factors (reviewed in Chapter 2). A link between PRC-function and DNA replication has been suggested before: Polycomb LOF in D.melanogaster, results in replication defect ${ }^{46}$. Hyperproliferation-associated replication damage and senescence are important features of oncogene induced senescence (OIS) and suggest a functional analogy. Chondrocytes appear to have a remarkable ability to hyper-replicate under conditions unfavorable for cell division (e.g. cartilage is extremely hypoxic). In addition, (pre)hypertrophic chondrocytes excrete vast amounts of ECM proteins that build and support the cartilaginous matrix. These conditions are known to induce endoplasmic reticulum (ER) stress signaling (ERSS) and the unfolded protein response (UPR); both ER stress and UPR are known inducers of OIS in the context of tumorigenesis ${ }^{47}$. ERSS/UPR and hypertrophic zone expansion are also at the basis for chondrodysplasia in mice and men ${ }^{47,48}$. The proposed biological analogy between hypertrophy and SLR is also consistent with a shared role for RUNX proteins in OIS and chondrocyte hypertophy ${ }^{11,37}$. These combined observations suggest that chondrogenic cells tolerate replication stress better than other cells. In agreement, we find that oncogenic RasV12 does not induce a replication arrest; in stead chondrogenic differentiation seems more robust. Indeed robust MAPK signaling is important in chondrogenesis ${ }^{49}$. PRC-LOF induces an exaggerated SASP/SMS response and blocks proliferation as a result of massive replication damage. Conversely, Bmi1 and RasV12 cooperate in oncogenesis ${ }^{50}$. Hence, transit amplification during chondrogenesis shares important characteristics of cellular responses to oncogenic activation and emphasizes a pivotal role for PRC1.

The findings reported here may point to a novel aspect of Polycomb biology during skeletogenesis: it is conceivable that proliferation and hence tissue expansion, co-determines positioning along the A-P axis and that premature termination of differentiation contributes to the PcG-phenotypes observed in null-mutants. Presence of SAbGAL in intervertebral disk cartilage possibly implicates a proliferative component in axial positioning. It is possible that abnormal HOX gene expression patterns contribute to the PRC1-phenotypes observed in null-mutants, both through direct effects on proliferation and differentiation. Indeed, recognition of a potential role for proliferation in axial patterning is long standing ${ }^{51}$. Combined our data suggest that PRC1 plays an important role in DNA damage avoidance in transit amplifying progenitor cells and that differentiation and senescence programs may be linked through DNA-damage responses. This then raises the interesting possibility that replication-mediated DNA-damage may also contribute to stem cell self- 
renewal defects, a possibility which appears to hold true for mesenchymal osteogenic progenitors in Bmi1-null mice ${ }^{52}$.

The findings presented herein consolidate a function for PRC1 at the cross road of transcription and replication. Our observations findings provide important connecting points for further research and have important implications for our mechanistic understanding of PRC1 proteins in embryogenesis and oncogenesis.

\section{Acknowledgements}

The authors thank H. Brock (Vancouver, Canada) and R.Shiri-Sverdlov (Maastricht, The Netherlands) for critically reading the manuscript. The authors received financial support from the European Molecular Biology Organization (Germany) ASTF5-2009; the Dutch Science Organization (ZonMW-NWO) VIDI grant 016.046.362, the National Rheuma Foundation (Reumafonds) grant LLP14 and transnational University of Limburg (tUL) grant to JWV.

\section{References}

1. Gunster, M. J. et al. Differential expression of human Polycomb group proteins in various tissues and cell types. J Cell Biochem Supp/ Suppl 36, 129-43 (2001).

2. Cao, R., Tsukada, Y. \& Zhang, Y. Role of Bmi-1 and Ring1A in H2A ubiquitylation and Hox gene silencing. Mol Cell 20, 845-54 (2005).

3. Cao, R. et al. Role of histone H3 lysine 27 methylation in Polycomb-group silencing. Science 298, 1039-43 (2002).

4. de Napoles, M. et al. Polycomb group proteins Ring1A/B link ubiquitylation of histone $H 2 A$ to heritable gene silencing and X inactivation. Dev Cell 7, 663-76 (2004).

5. Muller, J. et al. Histone methyltransferase activity of a Drosophila Polycomb group repressor complex. Cell 111, 197-208 (2002).

6. Akasaka, T. et al. Mice doubly deficient for the Polycomb Group genes Mel18 and Bmi1 reveal synergy and requirement for maintenance but not initiation of Hox gene expression. Development 128, 1587-97 (2001).

7. Suzuki, M. et al. Involvement of the Polycomb-group gene Ring1B in the specification of the anteriorposterior axis in mice. Development 129, 4171-83 (2002).

8. van der Lugt, N. M., Alkema, M., Berns, A. \& Deschamps, J. The Polycomb-group homolog Bmi-1 is a regulator of murine Hox gene expression. Mech Dev 58, 153-64 (1996).

9. Wang, J., Mager, J., Schnedier, E. \& Magnuson, T. The mouse PcG gene eed is required for Hox gene repression and extraembryonic development. Mamm Genome 13, 493-503 (2002).

10. Atsumi, T., Miwa, Y., Kimata, K. \& Ikawa, Y. A chondrogenic cell line derived from a differentiating culture of AT805 teratocarcinoma cells. Cell Differ Dev 30, 109-16 (1990).

11. Mackie, E. J., Ahmed, Y. A., Tatarczuch, L., Chen, K. S. \& Mirams, M. Endochondral ossification: how cartilage is converted into bone in the developing skeleton. Int J Biochem Cell Biol 40, 46-62 (2008).

12. Alkema, M. J., van der Lugt, N. M., Bobeldijk, R. C., Berns, A. \& van Lohuizen, M. Transformation of axial skeleton due to overexpression of bmi-1 in transgenic mice. Nature 374, 724-7 (1995).

13. Bel, S. et al. Genetic interactions and dosage effects of Polycomb group genes in mice. Development 125, 3543-51 (1998).

14. del Mar Lorente, M. et al. Loss- and gain-of-function mutations show a polycomb group function for Ring1A in mice. Development 127, 5093-100 (2000). 
15. Clarke, R. B. Isolation and characterization of human mammary stem cells. Cell Prolif $\mathbf{3 8}, \mathbf{3 7 5 - 8 6}$ (2005).

16. Pinto, D. \& Clevers, H. Wnt control of stem cells and differentiation in the intestinal epithelium. Exp Cell Res 306, 357-63 (2005).

17. Pommier, Y. et al. Repair of and checkpoint response to topoisomerase I-mediated DNA damage. Mutat Res 532, 173-203 (2003).

18. Sasaki, M., Ikeda, H., Sato, Y. \& Nakanuma, Y. Decreased expression of Bmi1 is closely associated with cellular senescence in small bile ducts in primary biliary cirrhosis. Am J Pathol 169, 831-45 (2006).

19. Itahana, K. et al. Control of the replicative life span of human fibroblasts by p16 and the polycomb protein Bmi-1. Mol Cell Biol 23, 389-401 (2003).

20. Jacobs, J. J., Kieboom, K., Marino, S., DePinho, R. A. \& van Lohuizen, M. The oncogene and Polycomb-group gene bmi-1 regulates cell proliferation and senescence through the ink4a locus. Nature 397, 164-8 (1999).

21. Bartkova, J. et al. Oncogene-induced senescence is part of the tumorigenesis barrier imposed by DNA damage checkpoints. Nature 444, 633-7 (2006).

22. Di Micco, R. et al. Oncogene-induced senescence is a DNA damage response triggered by DNA hyper-replication. Nature 444, 638-42 (2006).

23. Maertens, G. N. et al. Several distinct polycomb complexes regulate and co-localize on the INK4a tumor suppressor locus. PLoS One 4, e6380 (2009).

24. Agger, K. et al. The H3K27me3 demethylase JMJD3 contributes to the activation of the INK4A-ARF locus in response to oncogene- and stress-induced senescence. Genes Dev 23, 1171-6 (2009).

25. Coppe, J. P. et al. Senescence-associated secretory phenotypes reveal cell-nonautonomous functions of oncogenic RAS and the p53 tumor suppressor. PLoS Biol 6, 2853-68 (2008).

26. Bracken, A. P. et al. The Polycomb group proteins bind throughout the INK4A-ARF locus and are disassociated in senescent cells. Genes Dev 21, 525-30 (2007).

27. Jiang, L., Li, J. \& Song, L. Bmi-1, stem cells and cancer. Acta Biochim Biophys Sin (Shanghai) 41, 527-34 (2009).

28. Cui, H. et al. Bmi-1 regulates the differentiation and clonogenic self-renewal of I-type neuroblastoma cells in a concentration-dependent manner. J Biol Chem 281, 34696-704 (2006).

29. Fasano, C. A. et al. Bmi-1 cooperates with Foxg1 to maintain neural stem cell self-renewal in the forebrain. Genes Dev 23, 561-74 (2009).

30. He, S. et al. Bmi-1 over-expression in neural stem/progenitor cells increases proliferation and neurogenesis in culture but has little effect on these functions in vivo. Dev Biol 328, 257-72 (2009).

31. Merkerova, M., Bruchova, H., Kracmarova, A., Klamova, H. \& Brdicka, R. Bmi-1 over-expression plays a secondary role in chronic myeloid leukemia transformation. Leuk Lymphoma 48, 793-801 (2007).

32. Molofsky, A. V., He, S., Bydon, M., Morrison, S. J. \& Pardal, R. Bmi-1 promotes neural stem cell selfrenewal and neural development but not mouse growth and survival by repressing the p16Ink4a and p19Arf senescence pathways. Genes Dev 19, 1432-7 (2005).

33. Oguro, H. [Regulation of hematopoietic stem cell self-renewal by a polycomb group gene product, Bmi-1]. Rinsho Ketsueki 47, 363-70 (2006).

34. Knudsen, E. S., Buckmaster, C., Chen, T. T., Feramisco, J. R. \& Wang, J. Y. Inhibition of DNA synthesis by RB: effects on G1/S transition and S-phase progression. Genes Dev 12, 2278-92 (1998).

35. Fischer, S., Prykhozhij, S., Rau, M. J. \& Neumann, C. J. Mutation of zebrafish caf-1b results in S phase arrest, defective differentiation, and p53-mediated apoptosis during organogenesis. Cell Cycle 6, 2962-9 (2007).

36. Fasano, C. A. et al. shRNA knockdown of Bmi-1 reveals a critical role for p21-Rb pathway in NSC self-renewal during development. Cell Stem Cell 1, 87-99 (2007).

37. Kilbey, A., Terry, A., Cameron, E. R. \& Neil, J. C. Oncogene-induced senescence: an essential role for Runx. Cell Cycle 7, 2333-40 (2008).

38. Bracken, A. P., Dietrich, N., Pasini, D., Hansen, K. H. \& Helin, K. Genome-wide Mapping of Polycomb Target Genes Unravels Their Roles in Cell Fate Transitions. Genes and Development 20, 1123-36 (2006).

39. Wei, X. et al. Segregation of transcription and replication sites into higher order domains. Science 281, 1502-6 (1998).

40. Luo, L., Yang, X., Takihara, Y., Knoetgen, H. \& Kessel, M. The cell-cycle regulator geminin inhibits Hox function through direct and polycomb-mediated interactions. Nature 427, 749-53 (2004).

41. Ohtsubo, M. et al. Polycomb-group complex 1 acts as an E3 ubiquitin ligase for Geminin to sustain hematopoietic stem cell activity. Proc Natl Acad Sci U S A (2008). 
42. Orlando, V., Jane, E. P., Chinwalla, V., Harte, P. J. \& Paro, R. Binding of trithorax and Polycomb proteins to the bithorax complex: dynamic changes during early Drosophila embryogenesis. Embo $J$ 17, 5141-50 (1998).

43. Bernstein, B. E. et al. A bivalent chromatin structure marks key developmental genes in embryonic stem cells. Cell 125, 315-26 (2006).

44. Gilbert, D. M. Replication timing and transcriptional control: beyond cause and effect. Curr Opin Cell Biol 14, 377-83 (2002).

45. Azuara, V. et al. Chromatin signatures of pluripotent cell lines. Nat Cell Biol 8, 532-538 (2006).

46. O'Dor, E., Beck, S. A. \& Brock, H. W. Polycomb group mutants exhibit mitotic defects in syncytial cell cycles of Drosophila embryos. Dev Biol (2005).

47. Tsang, K. Y. et al. Surviving Endoplasmic Reticulum Stress Is Coupled to Altered Chondrocyte Differentiation and Function. PLoS Biology 5, e44 (2007).

48. Ho, M. S. P. et al. COL10A1 nonsense and frameshift mutations have a gain-of-function effect on the growth plate in human and mouse metaphyseal chondrodysplasia type Schmid. Hum. Mol. Genet., ddm067 (2007).

49. Stanton, L. A., Underhill, T. M. \& Beier, F. MAP kinases in chondrocyte differentiation. Dev Biol 263, 165-75 (2003).

50. Datta, S. et al. Bmi-1 Cooperates with H-Ras to Transform Human Mammary Epithelial Cells via Dysregulation of Multiple Growth-Regulatory Pathways. Cancer Res 67, 10286-10295 (2007).

51. Duboule, D. Vertebrate Hox genes and proliferation: an alternative pathway to homeosis? Curr Opin Genet Dev 5, 525-8 (1995).

52. Zhang, H. W. et al. Defects in Mesenchymal Stem Cell Self-Renewal and Cell Fate Determination Lead to an Osteopenic Phenotype in Bmi-1 Null Mice. J Bone Miner Res (2009).

53. R Development Core Team (2008). R: A language and environment for statistical computing. R Foundation for Statistical Computing, Vienna,

Austria. ISBN 3-900051-07- 0, URL http://www.R-project.org.

54. PMID: 15461798 - url: http://www.bioconductor.org/

55. PMID: 15175677

56. PMID: 18817533 - url: http://www.pathvisio.org

57. PMID: 18651794 - url: http://www.wikipathways.org 


\section{Supplemental Materials and methods}

\section{Cell culture}

ATDC5 cells were cultured at $37{ }^{\circ} \mathrm{C}, 5 \% \mathrm{CO} 2,100 \%$ humidity in DMEM/ F-12 supplemented with $5 \%$ fetal calf serum (FCS), antibiotics (100 units/ml penicillin and $100 \mu \mathrm{g} / \mathrm{ml}$ streptomycin), $200 \mathrm{mM}$ L-glutamine on tissue culture plates (Greiner Bio-One). For differentiation experiments cells were seeded at 6400 cells $/ \mathrm{cm}^{2}$ and were allowed to attach overnight. Growth medium was changed for differentiation medium, which includes ITS $\left(10 \mu \mathrm{g} / \mathrm{ml}\right.$ insulin, $10 \mu \mathrm{g} / \mathrm{ml}$ transferrin and $3 \times 10^{-8} \mathrm{M}$ sodium selenite). Differentiation medium was replaced every two days.

\section{Retroviral infections}

Ecotropic retroviral supernatants were produced by transfection of phoenix cells with calciumphosphate precipitation; 24-48 hours post-transfection, the supernatant was harvested, filtered and used for infection of ATDC5 cells in presence of $4 \mu \mathrm{g} / \mathrm{ml}$ polybrene. Cells were incubated for 12 hours and then allowed to recover for 24 hours on fresh medium before selection pressure was applied. Infected cells were selected with puromycin $(8 \mu \mathrm{g} / \mathrm{ml})$ for 72 hours, before experiments were initiated. Short-hairpin (sh)RNA target sequence for shBMI1hm 5'GTTCACAAGACCAGACCAC -3', shBMI1h 5'- GAATGGTCCACTTCCATTG-3', shRNF2 5'GTATCTGGCTGTGAGGTTA-3', shPHC2 5'- GTTCAAGCGTTCCAAGCGC-3'. Short hairpin sequence targeting shGFP was used as reference sh sequence ${ }^{57}$.

\section{Chrystal violet}

ATDC5 cells were plated in 12-multiwell plates (Greiner Bio-one). At each time point cells were washed twice with phosphate-buffered saline, and fixed for 10 minutes with $3.7 \%$ formaldehyde at room temperature. Next, cells were rinsed 5 times with demiwater. Cells were stained with $0.1 \% \mathrm{X}$ tal violet for 30 minutes or overnight, respectively, and washed 5 times with demiwater. X-tal was extracted with $10 \%$ acetic acid and absorbance was measured spectrophotometrically at $590 \mathrm{~nm}$ (Benchmark, Biorad).

\section{RNA isolation and cDNA synthesis}

Cells were suspended in Tri-reagent, and RNA was extracted according to the protocol of the manufacturer (Sigma). Quantity and purity of the RNA were determined by 260/280 nm absorbance measurements. cDNA was synthesized using iScript ${ }^{\mathrm{TM}} \mathrm{CDNA}$ synthesis kit (Biorad). Primers used are described in supplemental figure 2.

\section{Western analysis}

Cells were lysed in RIPA buffer containing $5 \mathrm{mM}$ Benzamidine, $5 \mu \mathrm{g} / \mathrm{ml}$ Antipain, $5 \mu \mathrm{g} / \mathrm{ml}$ Leupeptin, $5 \mathrm{\mu g} / \mathrm{ml}$ Aprotinin, $1 \mathrm{mM}$ Sodium Vanadate, $10 \mathrm{mM}$ Sodium Fluoride, $10 \mathrm{mM}$ Pyrophosphate, $10 \mathrm{mM}$ ß-glycerophosphate, $0.5 \mathrm{mM}$ DTT and 1mM PMSF. Lysates were 


\section{Chapter 5}

subjected to 3 freeze-thaw cycles in liquid nitrogen, and to 3 cycles of sonication. After centrifugation for 10 minutes at $13.2 \mathrm{k} \mathrm{rpm}$, protein concentration was determined using a $\mathrm{BCA}$ protein assay kit (Pierce). Equal amounts of protein were boiled in Laemmli buffer and loaded on 9$15 \%$ polyacrylamide gels. Proteins were transferred to polyvinylidene fluoride (PVDF) membranes. After blocking with $5 \%$ non-fat dry milk in PBS containing $0.1 \%$ Tween-20, membranes were incubated $\mathrm{o} / \mathrm{n}$ at $4^{\circ} \mathrm{C}$ with the following antibodies: anti $\beta$-actin Mab (C4, 691001, MP Biomedicals), anti Gapdh, anti Ezh2 Mab (BD43; coutesy D. Pasin Copenhagen, Denmark), anti Bmi1 Mab (F6; Courtesy M. van Lohuizen; Amsterdam, The Netherlands), anti $\gamma \mathrm{H} 2 \mathrm{~A} . \mathrm{X}$ (Ser139) Poab \#2577 (Cell signaling), anti H3K27me3 Poab, \#07-449 (Upstate) and anti H3K9me3 \#07-442 (Upstate). After extensive washing, membranes were incubated with corresponding horseradish peroxidase conjugated secondary antibodies for $1 \mathrm{~h}$ at room temperature. Signals were detected using enhanced chemoluminescence.

\section{Immunofluorescence}

Briefly, cells were washed and fixed for $10-15$ minutes in $100 \%$ methanol at $-20^{\circ} \mathrm{C}$ and stored at $4^{\circ} \mathrm{C}$ in $70 \%$ ethanol or used directly for immuno-histochemistry. Cells were permeabilized for $5-10$ minutes in $0.2 \&$ triton-X in PBS. To prevent epitope loss in combination with acid treatment for $\mathrm{BrdU}$ detection, primary antisera were incubated for 1.5 hours at $37^{\circ} \mathrm{C}, 4-5 \mathrm{x}$ washed in $0.02 \%$ triton-X/PBS and fixed in $2 \%$ formaldehyde/PBS for 10 minutes at room temperature. Cells were washed with PBS and incubated for 20 minutes in $2.0 \mathrm{~N} \mathrm{HCl}$ at $37^{\circ} \mathrm{C}$, followed by two rinses of 0.1 $\mathrm{M}$ sodium tetraborate solution, $\mathrm{pH} 8.5$ for in total 2 minutes. Cells were than incubated with the primary antisera against BrdU, washed at indicated before and incubated with secondary fluorescently labeled antibodies. All Antisera were diluted and incubated in $0.02 \%$ triton-X in PBS. Nuclei were counterstained with 4'-6-Diamidino-2-phenylindole (DAPI) and washed in $0.02 \%$ tritonX/PBS. The last wash step was in PBS, upon which cells were dehydrated: 1 minute in $70 \%$ ethanol, 2x 1 minute in 100\% ethanol and air-dried. Cells were mounted in Vectashield (Vector Laboratories, Inc. Burlingame, CA) and analyzed using a NIKON TE200 Eclipse fluorescence microscope and photographed using a NIKON DXM1200 digital camare in combination with NIS Elements 3.0 Imaging software. The following antibodied were used: anti YH2A.X Mab JBW301 (Upstate), anti BrdU (BD biosciences), anti H3K9me3 \#07-442 (Upstate), anti H3K27me3 Poab, \#07-449 (Upstate), anti Bmi1 Mab (F6; Courtesy M. van Lohuizen; Amsterdam, The Netherlands), anti H3S10Ph Poab \#06-570 (Upstate), anti Pcna PC10 Poab (Chemicon International), anti Phc1 (03041; Courtesy of H. Koseki), anti pRNA polymerase II Mab, ab24759 (Abcam), anti Rad51 Poab sc-8349 (Santa Cruz), anti pKap1 (S824) Poab A300-767A (Bethyl Laboratories), anti RPA70 Poab ab12320 (Abcam)an ant Geminin Poab (FL-209) sc-13015 (Santa Cruz). 


\section{Affymetrix espression arrays}

A total of 48 Affymetrix NuGO mouse arrays ( $24 k)$ were run on RNA from cell cultures sampled over time at $0,2,4,8$ hours and 1, 6, 10, 15 days post induction of differentiation (pid). For each time point 3 samples were taken from both a wildtype (WT) and a Bmi1 knock-down (KD) culture. After scanning, data preprocessing and analysis were done using R2.7.1 with the use of Bioconductor libraries ${ }^{53,54}$. First, data were ${ }^{2} \log$ transformed and normalized using gcRMA ${ }^{55}$. Hereafter several statistics were computed for each reporter on the arrays: the average ( ${ }^{2}$ logged) expression for each group at each time point, the logratio ( ${ }^{2} \log$ fold change, logFC) between the two groups at each time point, and the $\mathrm{t}$ - and $\mathrm{p}$ - value of a Student t-test at each time point. After computing statistics, subsets of reporters fulfilling specific criteria based on these statistics were selected for the several analyses, where generally cut-offs on p-value, logratio, and minimum expression were imposed and controls removed from the data set. Unless otherwise specified, the cut-off limits had to be met at either 1day pid or 6 days pid. Specific cut-off limits chosen are indicated where applicable in the paper. Overrepresentation of biological processes was determined using PathVisio, using the pathways available from WikiPathways ${ }^{56,57}$. 
A
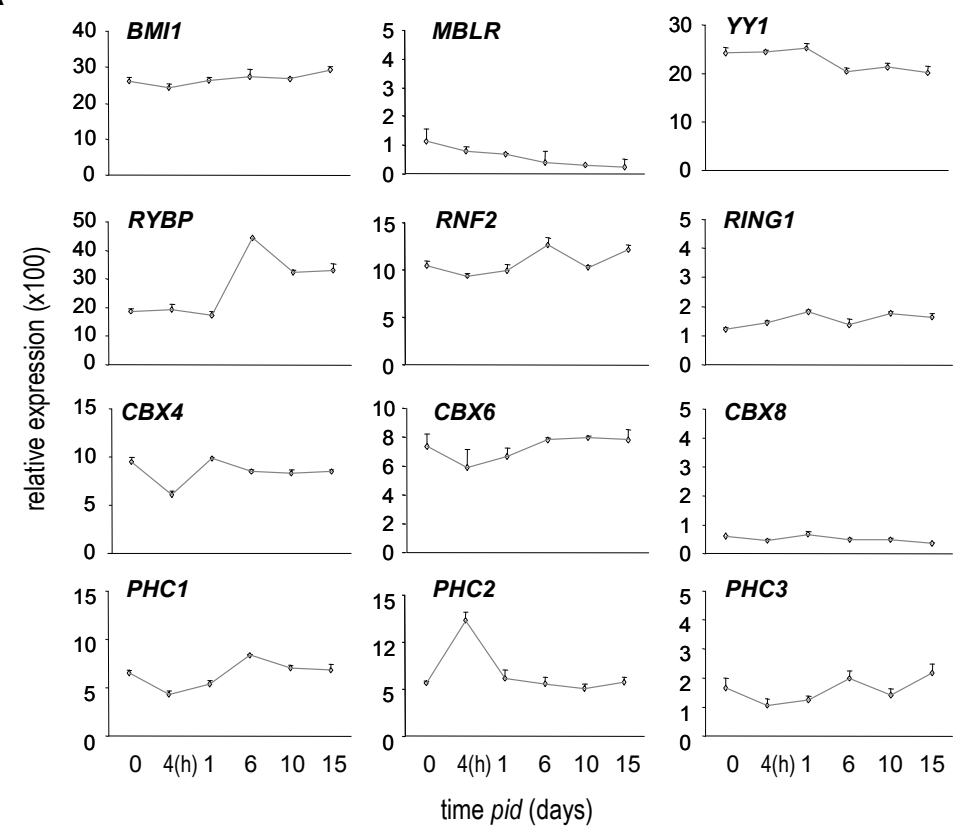

RING1

B

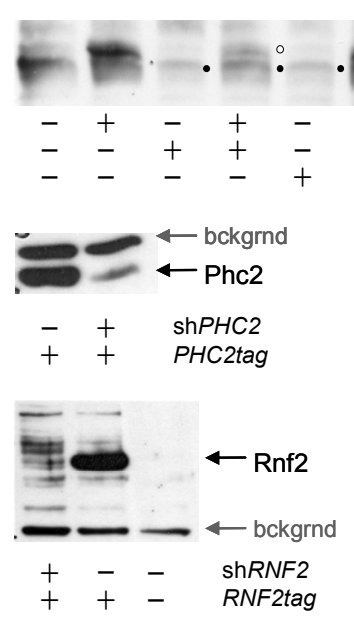

C

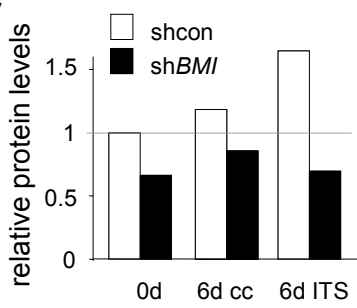

D

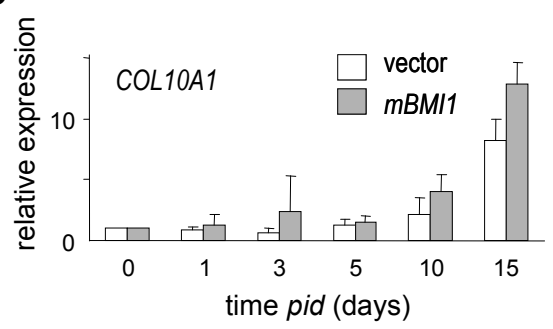

Figure S1. Expression analysis of PRC1 complex genes.

(A) mRNA expression of PRC1 complex genes during chondrogenesis by microarray analysis. (B) Western blots confirming functionality of short hairpins designed to target PcG mRNAs for RNAi mediated degradation (C) quantification of Bmi1 protein levels shown in figure 1b. (D) qRT rtPCR analysis of collagen 10A1 mRNA expression during differentiation in ATDC5 cells overexpressing Bmi1 and control cells. 
A

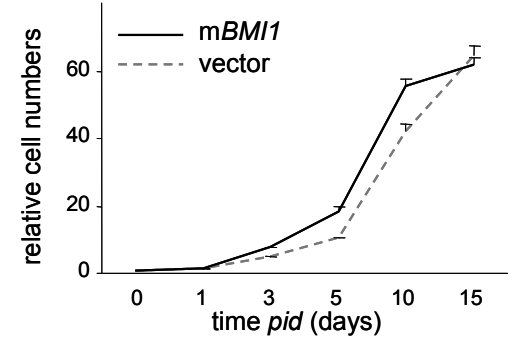

B

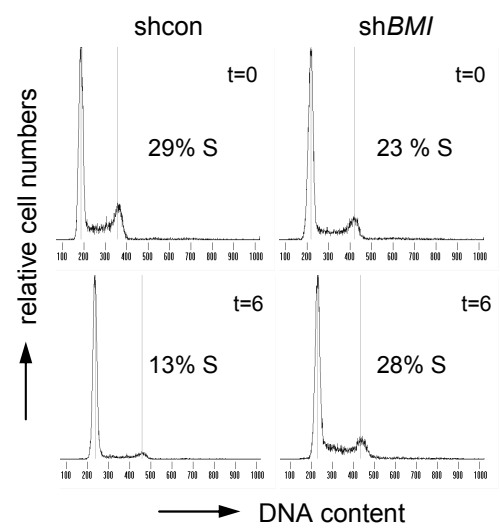

D

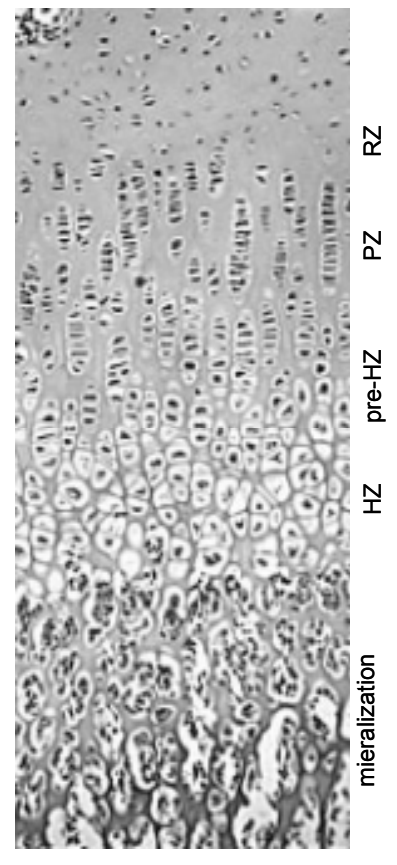

C
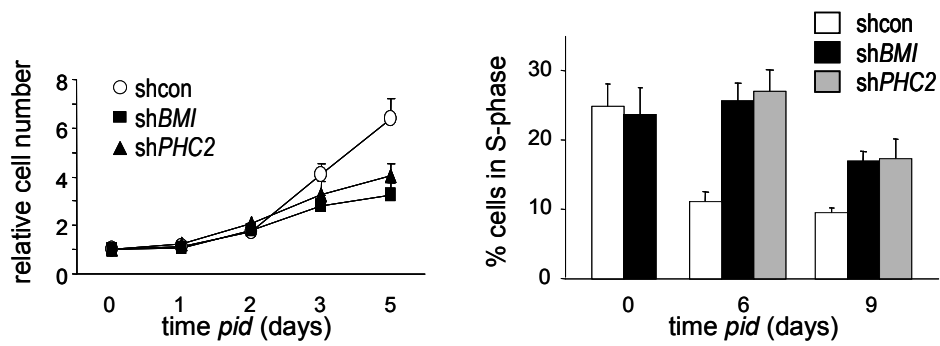

Figure S2. PRC1-LOF cells accumulate in S-phase during transit amplification.

(A) ATDC5 cells overexpressing Bmi1 show enhanced hyperproliferation upon induction of chondrogenic differentiation compared to control cells. Proliferation determined by Crystal violet incorporation with subsequent extraction and spectrophotometric analysis. (B) Representative cell cycle profiles of ATDC5 shControl and shBmi1 at $t=0$ and $t=6 \mathrm{~d}$ pid. Values are percentage of S-phase cells compared to total cell population, in addition quantification S-phase positive cells of total experiment. (C) Proliferation of ATDC5 cells expressing shControl, shBmi1 and shPhc2 upon induction of chondrogenic differentiation. Proliferation determined by Crystal violet incorporation with subsequent extraction and spectrophotometric analysis. (D) overview of growthplate. RZ= Resting Zone, PZ= Proliferation Zone, pre-HZ= preHypertrophic Zone, $\mathrm{HZ}=$ Hypertrophic Zone, the transition from cartilage to bone is located between $\mathrm{HZ}$ and mineralization. 
A

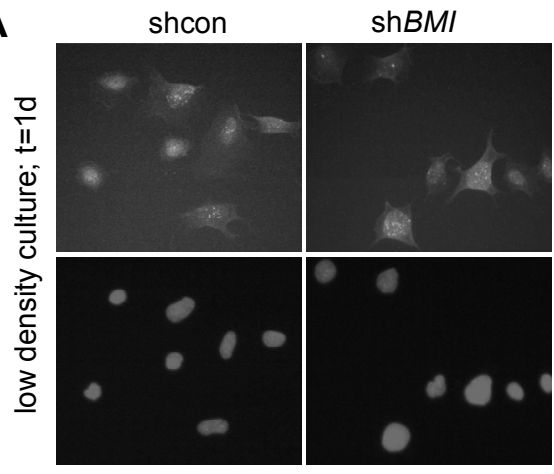

\section{B}

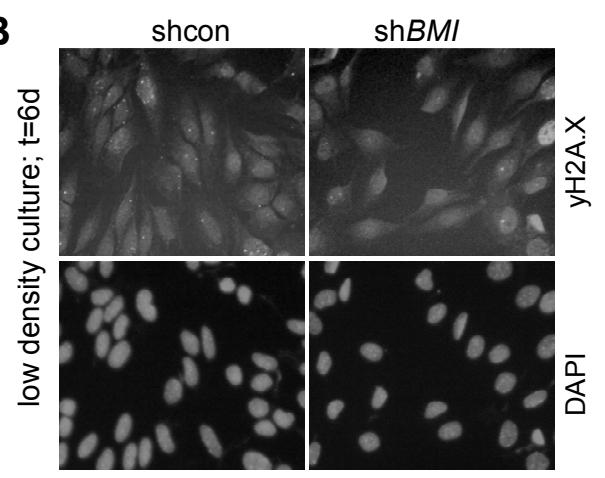

C

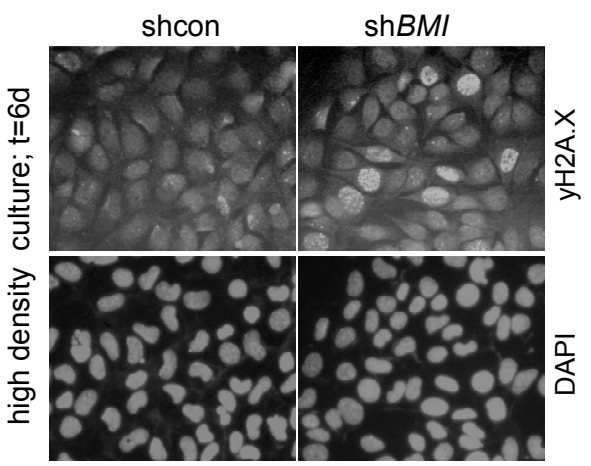

D

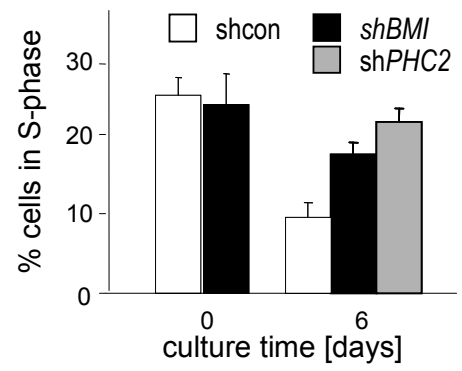

$\mathbf{E}$

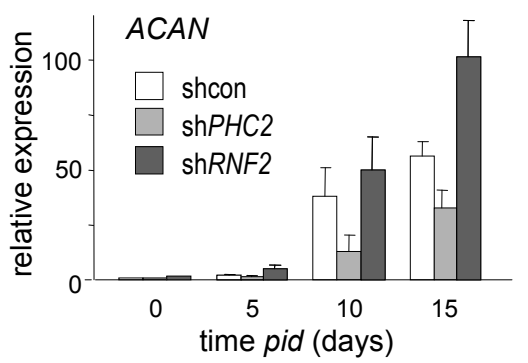

Figure S3. Accumulation of DNA damage in shBmi1 cultures.

(A, B, C) Immunofluorescence staining for $\mathrm{YH} 2 \mathrm{~A}$.X in ATDC5 shControl and shBmi1 cultures at varying densities. DAPI used as nuclear staining. (D) Quantification of S-phase positive cells in ATDC5 shControl, shBmi1 and shPhc2 cultures 0 and 6 days pid. (E) ACAN mRNA expression measured by qRT rtPCR in ATDC5 shControl, shBmi1 and shPhc2 throughout differentiation. 
A

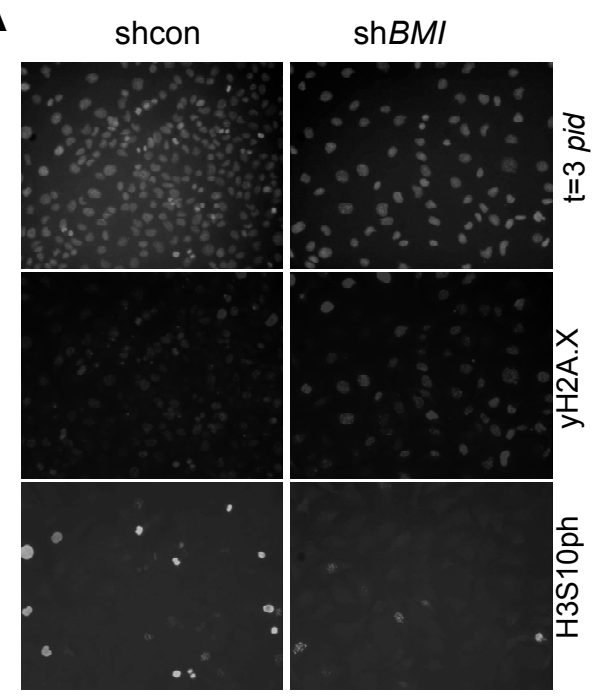

C

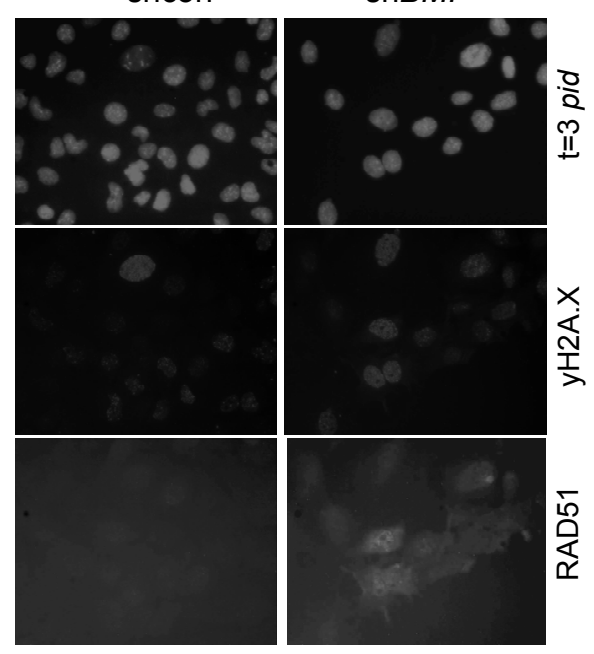

B

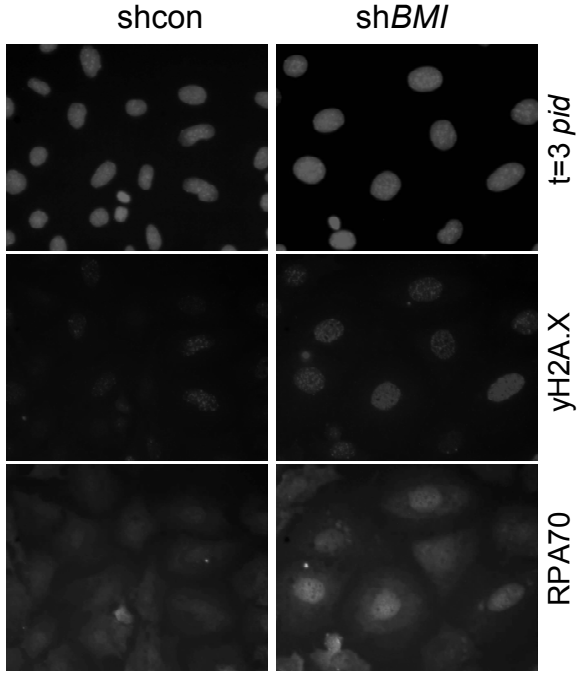

D

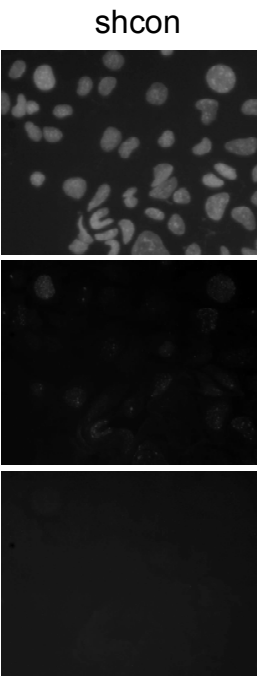

$\operatorname{sh} B M I$

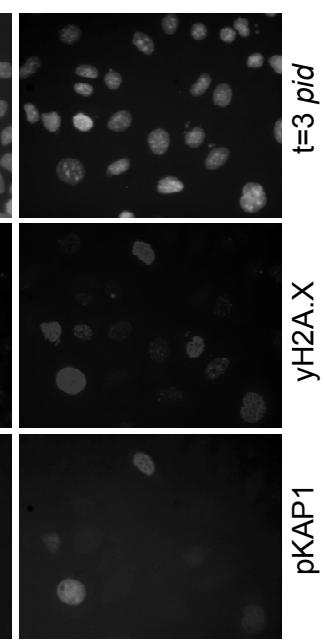

Figure S4. Bmi1-LOF results in activation of DNA damage responses and correlates to proliferative arrest. (A-D) Immunofluorescence analysis for indicated proteins in ATDC5 shControl and shBmi1 at $\mathrm{t}=3$ pid. DAPI is used as nuclear staining. 


\section{Chapter 5}

\begin{tabular}{|c|c|c|c|}
\hline Geneacronym & fold & description & Processesinvolved in \\
\hline Ifif204 & 4,9 & interferon activated gene 204 & tracri///tracri///tracriPol IV// transport //II// DDR ///Diff \\
\hline E130016E03Rik & 4,1 & RIKEN cDNA E130016E03 gene & Dre///DDR \\
\hline Brip1 & 3,5 & BRCA1 interacting protein (erminal helicase 1 & Nucmet///Dre/// tracriPol IV// DDR \\
\hline Fancb & 2,5 & Fanconi anemia, complementation group $B$ & Drel//DDR \\
\hline Rad9 & 2,4 & RAD9 homolog (S. pombe) & CC ///CC checkpoint //DDC ///Dre///DDR///RadR///apop \\
\hline Pttg1 & 2,3 & pituitary tumotransforming 1 & Dmet///Dre///DDR///CC ///Cseg///mit///Cseg///biog \\
\hline Rad51 & 2,2 & RAD51 homolog & homR///Dmet//REP///Dre///DDR///mei///meiR \\
\hline Hspa1b & 2,2 & heat shock protein $1 \mathrm{~B}$ & TELm/// Drel// fold /// antiapop //UPRR//hs /// \\
\hline Xrcc2 & 2,1 & X-ray repair complementing defective repair & Dmet///Dre///Dred//IDR \\
\hline Rad51ap1 & 2,1 & RAD51 associated protein 1 & homR///Dre///Dred//IDDR \\
\hline Ddb2 & 2,0 & damage specific DNA binding protein 2 & Dre/// pyrimidine dimer repair IDDR \\
\hline Exo1 & 1,9 & exonuclease 1 & Nucmet///Dre/// NER///MMR///Dred//DDR///mei \\
\hline $\mathrm{Blm}$ & 1,9 & Bloom syndrome homolog (human) & REP/// Drel//Dred/l \\
\hline Eme1 & 1,8 & essential meiotic endonuclease 1 homolog 1 & Dre///Dred//IDRR \\
\hline Chaf1a & 1,8 & chromatin assembly factor 1 , subunit A (p150) & REP/// Drel// tracri///tracri/// fold /// DDR///CC \\
\hline Sgk & 1,8 & serum/glucocorticoid regulated kinase & kin///apop ///DDR \\
\hline Hspa1a & 1,8 & heat shock protein $1 \mathrm{~A}$ & TELm/// Dre/// fold ///UPR///hs \\
\hline Brca2 & 1,8 & breast cancer 2 & homR///Dre/// chrom///DDR ///S-CC ///mitC///tracri \\
\hline Brca2 & 1,8 & breast cancer 2 & homR///Dre/// chrom///DDR ///S-CC ///mitC///tracri \\
\hline Rad5111 & 1,8 & RAD51like 1 & Dmet//Dre///Dred//DDR \\
\hline Cdc2a & 1,7 & div cycle 2 homolog A & kin/// antiapop ///CC ///mit///G2-C/// div \\
\hline Trex1 & 1,7 & three prime repair exonuclease 1 & REP///Drel//MMR///Dred///DDR \\
\hline Fen1 & 1,7 & flap structure specific endonuclease 1 & REP/// DNA repair \\
\hline Msh3 & 1,6 & mutS homolog 3 & Dmet//Drel//MMR///DDR///somH///somR \\
\hline Gtf2h1 & 1,6 & generaltracrifactor II H, polypeptide 1 & Drel//tracri///tracri/// DDR \\
\hline Clspn & 1,6 & claspin homolog & DRC///Dre///DDR///CC \\
\hline Rad50 & 1,5 & RAD50 homolog & Dmet///Dre///DDR///CC ///mei \\
\hline Mapk1 & 1,5 & mitogen activated protein kinase 1 & kin///DDR///CC ///ST///morf/// kin ///kin /// cytosinemet/// diff \\
\hline Topors & 1,4 & topoisomerase I binding, arginine/serimich & Ubd///apop ///DDR///met/// prol// tracri///trapo \\
\hline Lig1 & 1,4 & ligase I, DNA, ATPdependent & REP/// Dre/// Dred//DDR///CC /// div \\
\hline Gadd45a & 1,4 & growth arrest and DNAdamageinducible 45 alpha & regulation of progression throug $6 \mathrm{C} / / / \mathrm{Pase} / / / \mathrm{DDR} / / / \mathrm{CC} / / / \mathrm{CC}$ arrest \\
\hline Rfc5 & 1,4 & replication factor C (activator 1) 5 & REP/// DNA repair \\
\hline Eef1e1 & 1,4 & eukaryotictral elongation factor 1 epsilon 1 & CC ///Drel// tral///arr/// embrt//apop ///DDR \\
\hline Gt2h2 & 1,4 & generaltracrifactor II H, polypeptide 2 & Drel//tracri///tracri/// DDR///tracri \\
\hline Xab2 & 1,3 & XPA binding protein 2 & blast///Dre///TCR///tracri///Rproc \\
\hline Uvrag & 1,3 & UV radiation resistance associated gene & Dre \\
\hline Fanca & 1,3 & Fanconi anemia, complementation group A & Dre///DDR/// malemei/// Mgor/// Fgonl//prol \\
\hline Bre & 1,3 & brain and reproductive orgaexpressed protein & Ubd//apop///DDR///antiapop \\
\hline Pold1 & 1,3 & polymerase (DNA directed), delta 1, catalytic subunit & S-CC ///Dre///REP///BER///REP \\
\hline Topbp1 & 1,3 & topoisomerase (DNA) II beta binding protein & Dre///DDR///meiR \\
\hline Msh5 & 1,3 & mutS homolog 5 & Dmet//MMR///mei///meil ///syn /// Fgam \\
\hline Hmgb2 & 1,3 & high mobility group box 2 & REP/// REP/// Drel///BER/// chrom/// nuc/// tracri///tracriPol II \\
\hline Parp2 & 1,3 & poly (ADRribose) polymerase family, member 2 & Drel//BER/// ribos \\
\hline Smc3 & 1,2 & structural maintenace of chromosomes 3 & Dmet///Dre///DDR ///CC ///spin///Cseg///SCC///mit///mei///ST///biog///div \\
\hline Smc5 & 1,2 & structural maintenance of chromosomes 5 & Dmet///Dre///Dred//DDR \\
\hline
\end{tabular}

Table S1. Activation of DNA damage response in Bmi1-LOF cultures.

Expression array analysis of DNA damage response genes presented as log fold change. Associated processes based on Genmapp. 


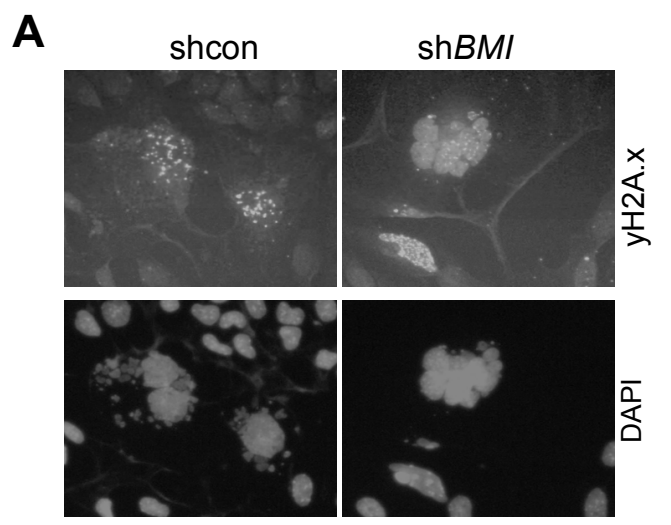

\section{B}
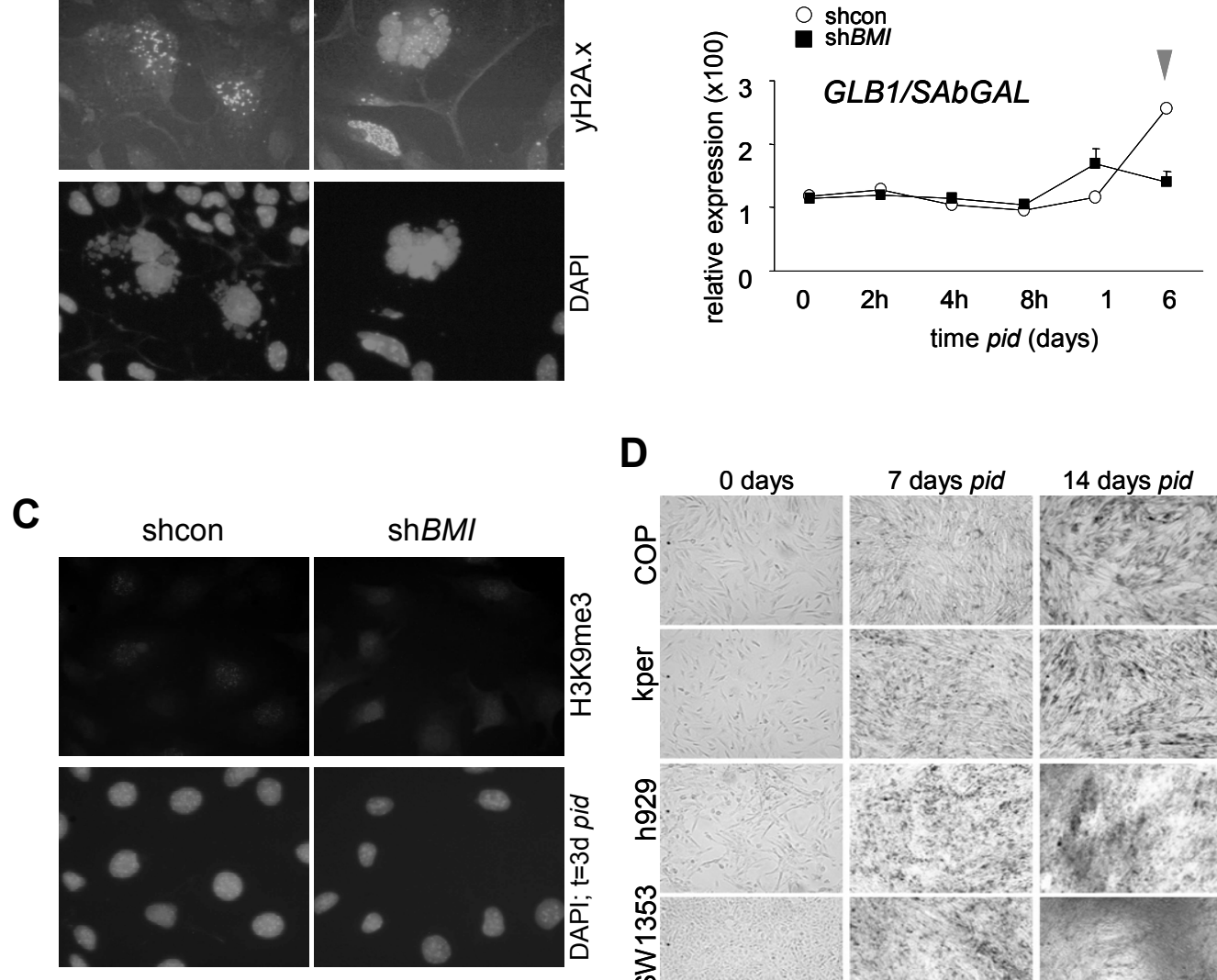

D

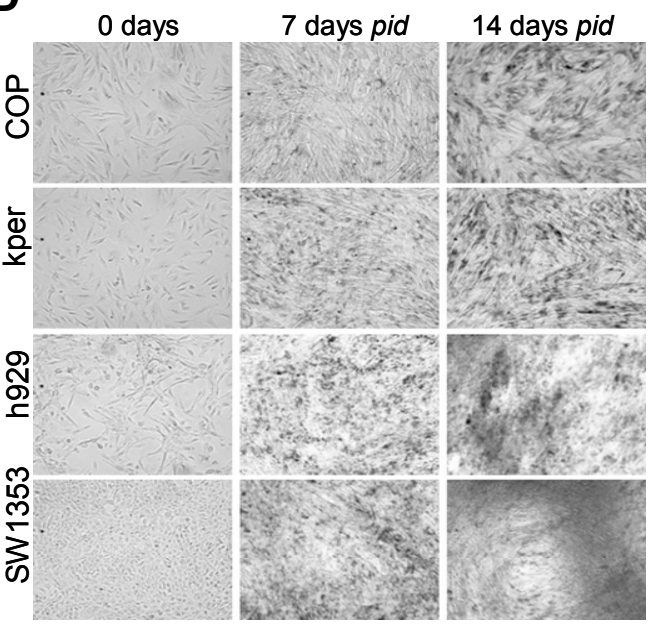

\section{F}

E

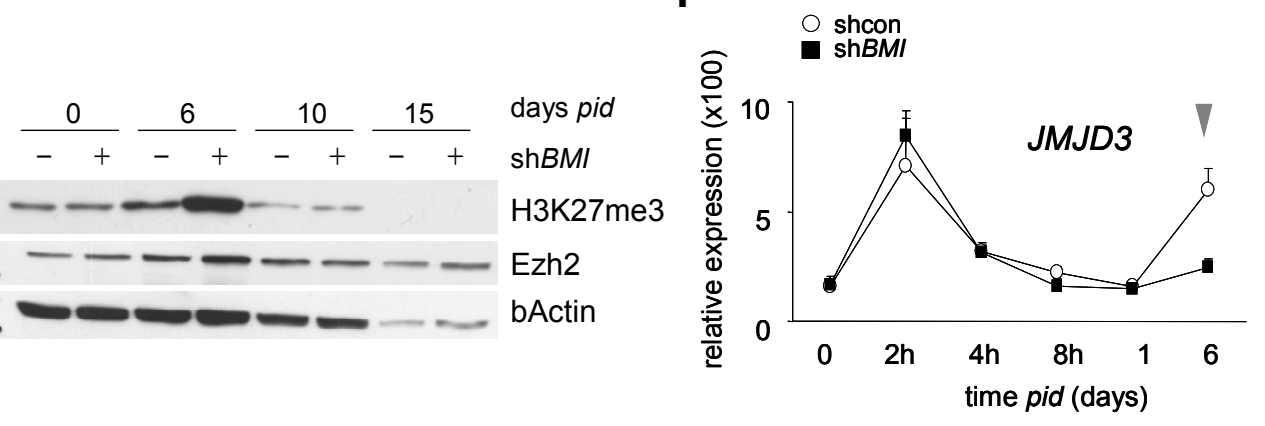



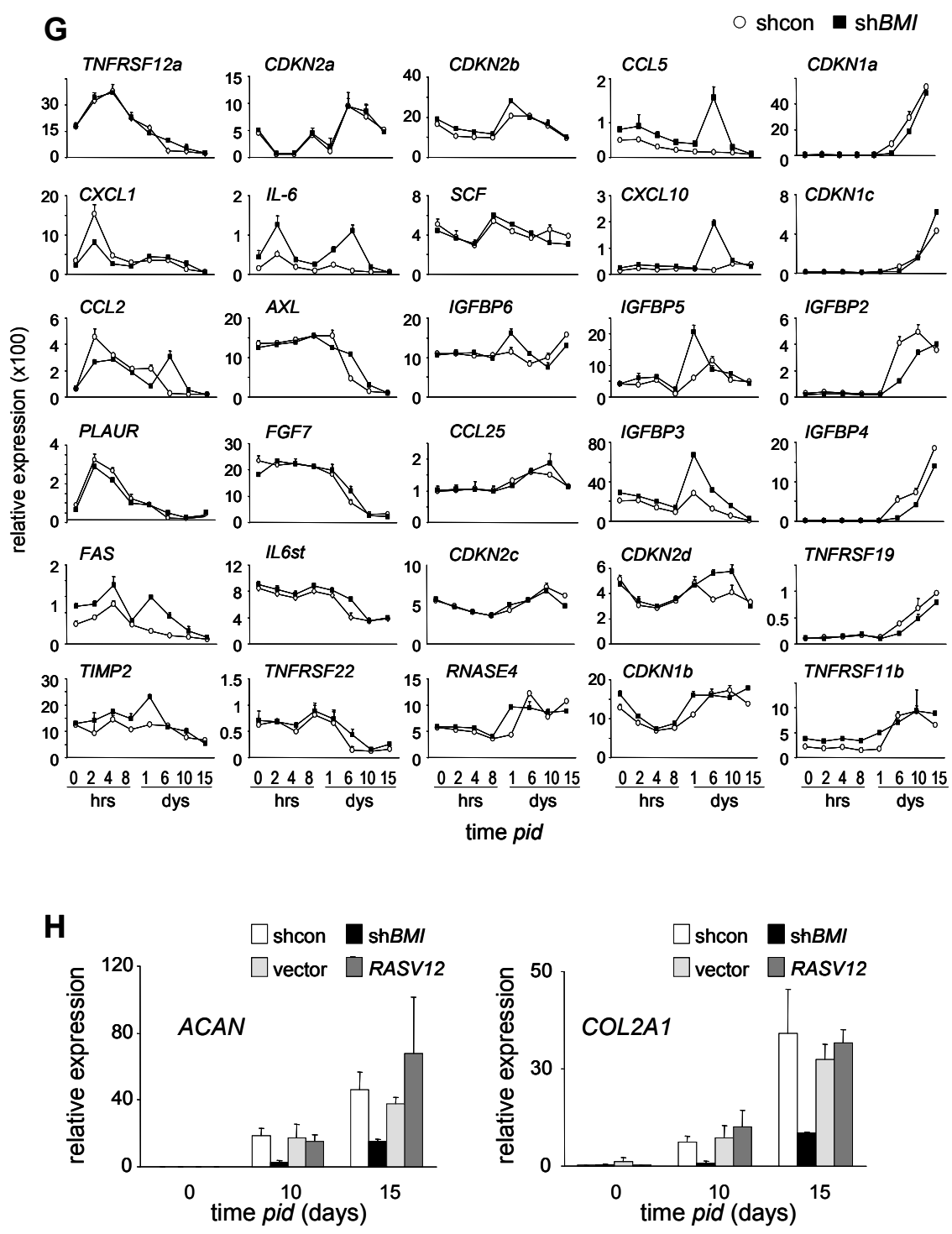

Figure S5. DNA damage and senescence in chondrogenesis.

$(A+C)$ Immunofluorescence analysis in ATDC5 shControl and shBmi1 of $\mathrm{yH} 2 \mathrm{~A} . \mathrm{X}$ and $\mathrm{H} 3 \mathrm{~K} 9 \mathrm{me} 3$ at $\mathrm{t}=3 \mathrm{~d}$ pid. DAPI is use as nuclear staining. (B,F) Expression array analysis of GLB1 and JMJD3 in ATDC5 shControl and shBmi1 during differentiation. (D) Senescence-associated bGalactosidase staining on chondrogenic cell lines. (E) Western blot analysis of H3K27me3 and Ezh2 in ATDC5 shControl and shBmi1 during differentiation. bActin is used as a loading control. (G) Expression array analysis of senescence associated genes in ATDC5 shControl and shBmi1 during differentiation. $(\mathrm{H})$ qRT rtPCR analysis of mRNA expression of chondrogenic markers in ATDC5 expressing shControl, shBmi1, vector or Rasv12 throughout differentiation. 
A

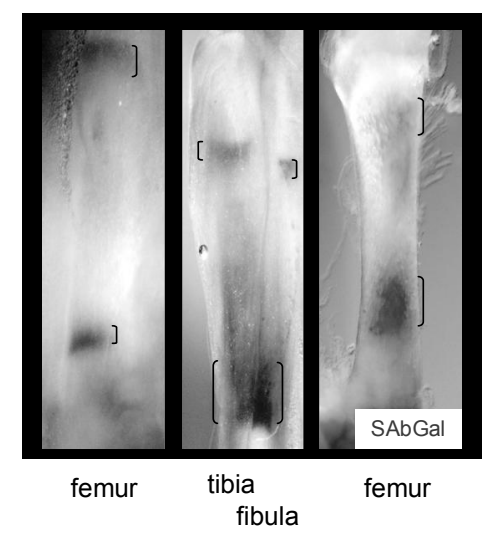

B

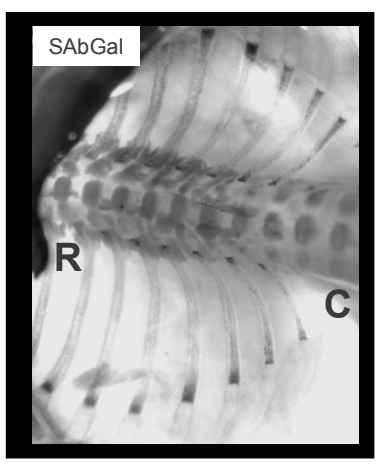

rib cage/spinal column ventral view

\section{C}
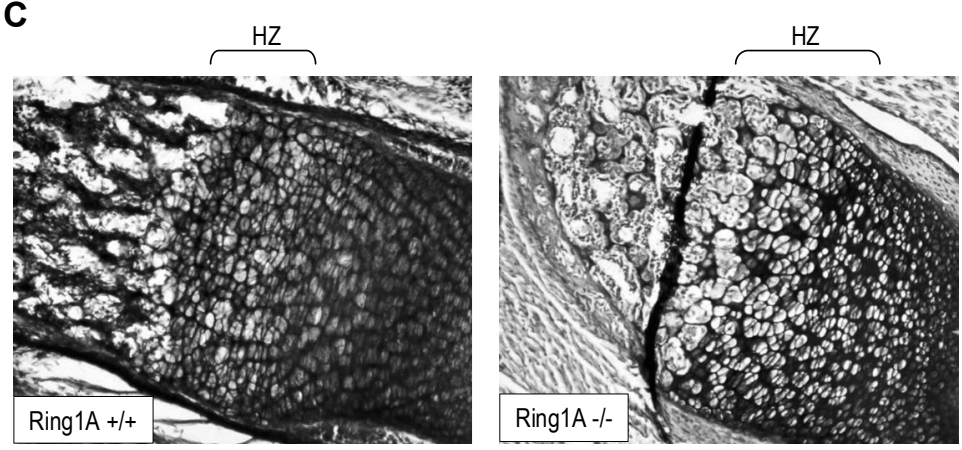

femurs

Figure S6. Growth plates of longitudinal bones are positive for SAbgal.

$(A, B)$ Representative senescence-associated bGalactosidase staining on longitudinal bones and ribcage/spinal column of wild-type mice. (C) Representative pictures of femur growth plates of Ring1a+/+ and Ring1a -/- mice. Brackets indicate hypertrophic zone. 
A

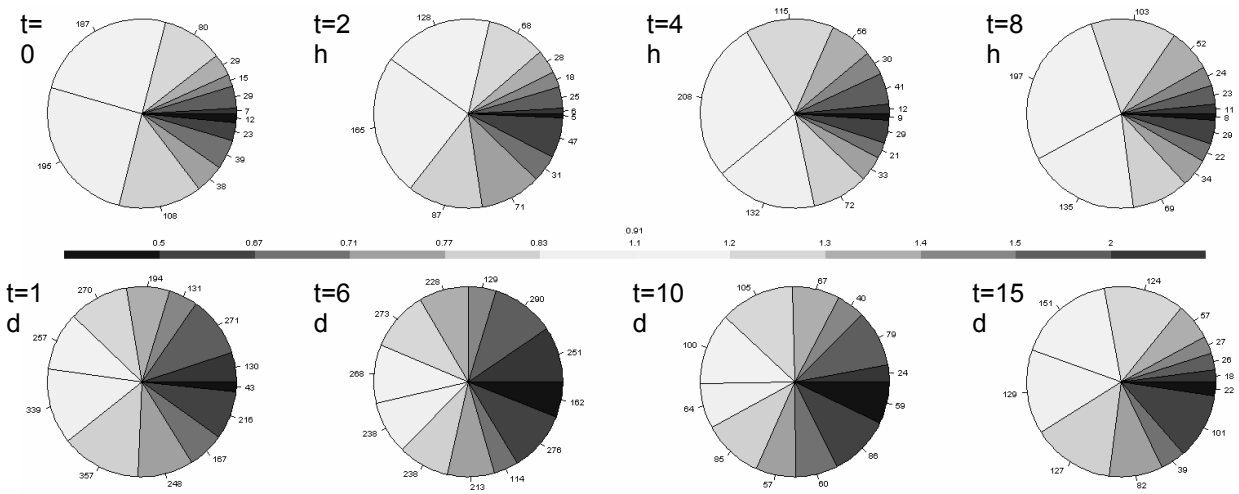

B

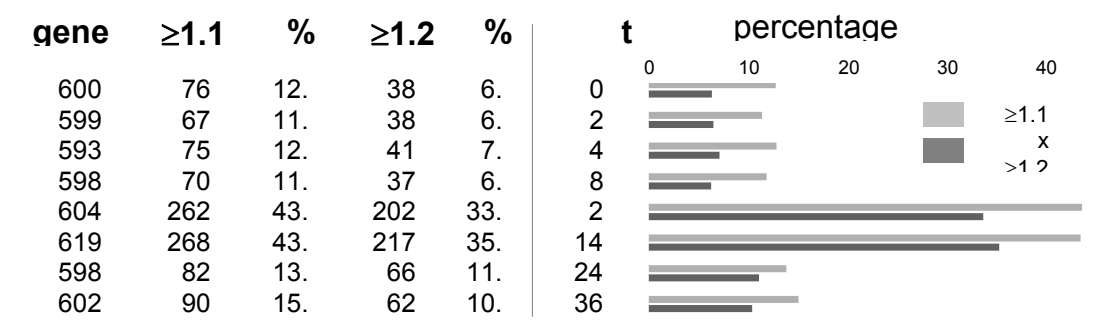

C

upregulated

downregulated
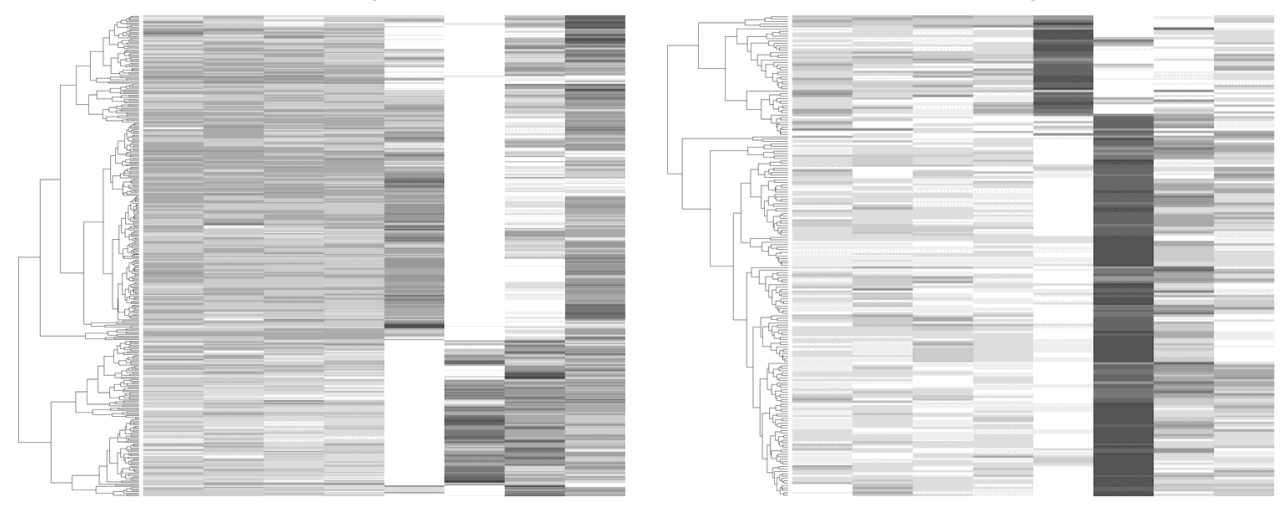
D a) consistently up in $K D$
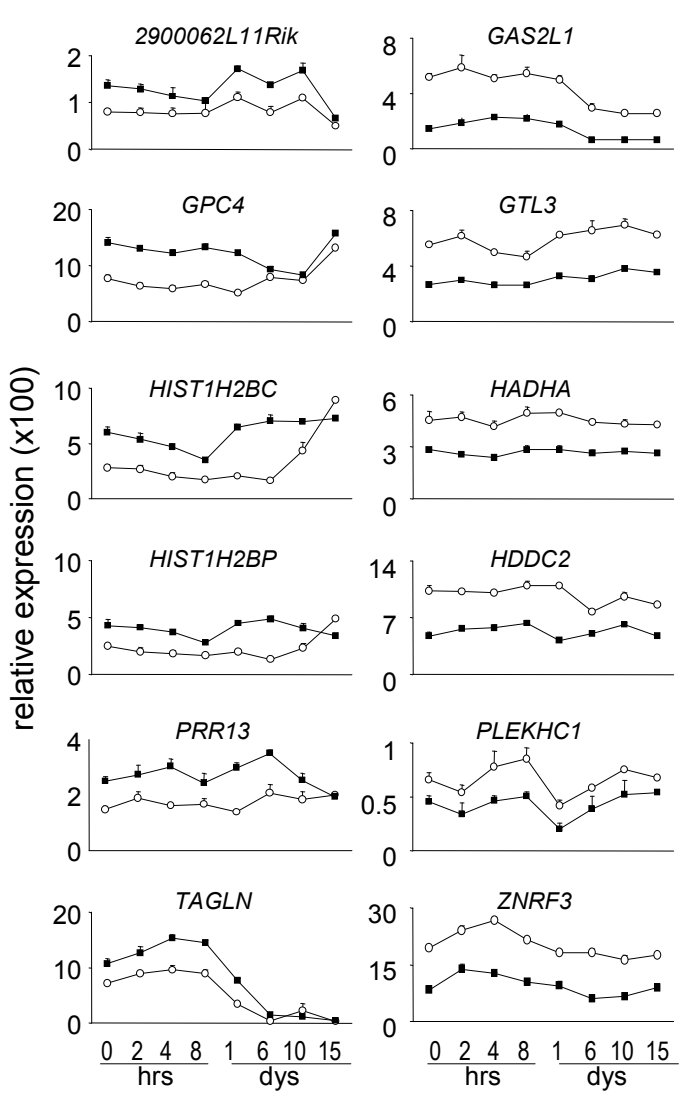

b) consistently down in $\mathrm{KD}$
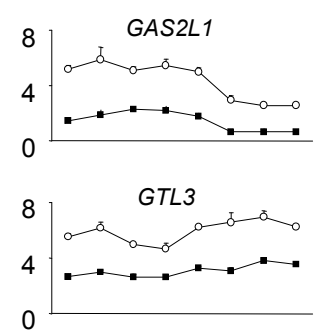

0
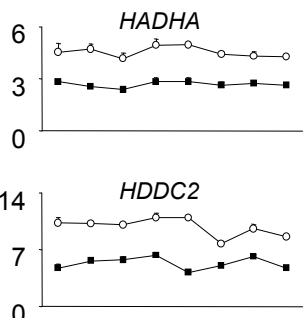

PLEKHC1 c) up - aberrantly induced in $\mathrm{KD}$ d) down - not induced in KD shcon $\mathbf{s h B M I}$ DDEF1
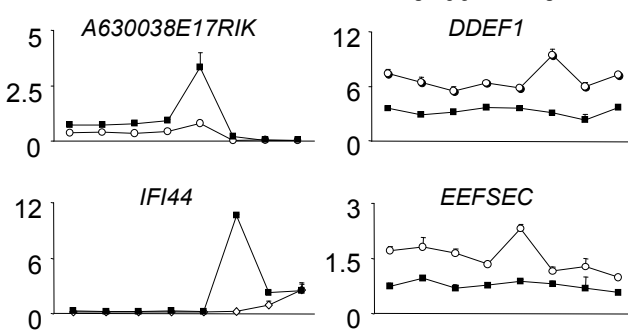

3. EEFSEC
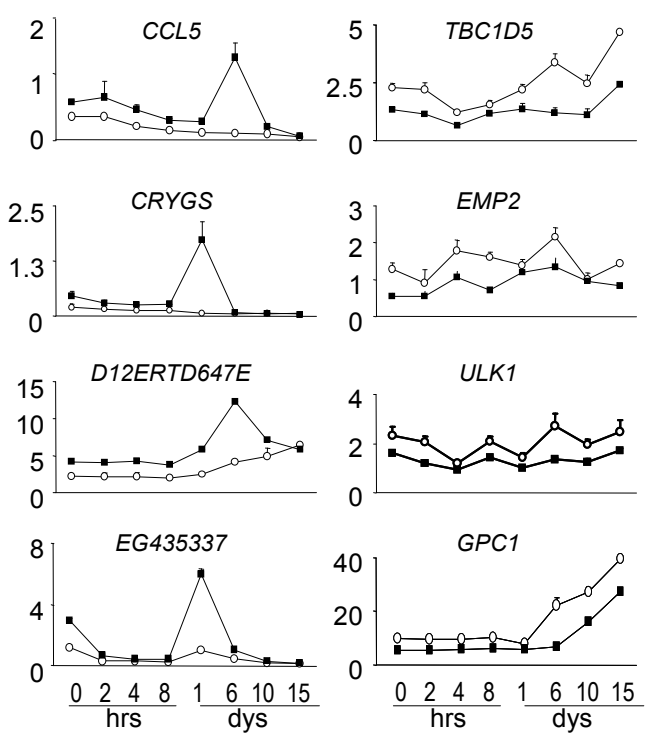

time pid

E

\begin{tabular}{|l|c|c|c|c|c|}
\hline Pathway & $\mathbf{r}$ & $\mathbf{n}$ & total & $\mathbf{\%}$ & $\mathbf{Z}$ \\
\hline Cholesterol Biosynthe sis & 10 & 13 & 15 & 76,9 & 4,66 \\
\hline Cell cycle & 32 & 70 & 88 & 45,7 & 4,62 \\
\hline TGF-beta Receptor Signaling Pathway & 44 & 115 & 149 & 38,3 & 4,03 \\
\hline Androgen Receptor Signaling Pathway & 33 & 84 & 108 & 39,3 & 3,65 \\
\hline Irinotecan Pathway & 7 & 10 & 13 & 70,0 & 3,56 \\
\hline G1 to S cell cycle control & 22 & 51 & 64 & 43,1 & 3,49 \\
\hline Endochondral Ossification & 23 & 56 & 68 & 41,1 & 3,29 \\
\hline TGF Beta Signaling Pathway & 18 & 41 & 52 & 43,9 & 3,24 \\
\hline mRNAprocessing & 102 & 349 & 552 & 29,2 & 3,06 \\
\hline Mm Selenium metabolism/Selenoproteins & 18 & 43 & 49 & 41,9 & 3,00 \\
\hline Apoptosis Modulation by HSP70 & 9 & 17 & 18 & 52,9 & 2,97 \\
\hline TNF-alpha/NF-kB Signaling Pathway & 47 & 143 & 177 & 32,9 & 2,94 \\
\hline DNAReplication & 15 & 36 & 41 & 41,7 & 2,71 \\
\hline Heme Bios ynthesis & 5 & 8 & 9 & 62,5 & 2,68 \\
\hline One Carbon Metabolism & 10 & 23 & 41 & 43,5 & 2,37 \\
\hline Eukaryotic Transcription Initiation & 15 & 39 & 41 & 38,5 & 2,35 \\
\hline Mitochondrial LC-Fatty Acid Beta-Oxidation & 6 & 13 & 16 & 46,2 & 2,01 \\
\hline Apoptosis Mechanisms & 24 & 74 & 86 & 32,4 & 2,00 \\
\hline
\end{tabular}


$\mathbf{F}$

pathways

Mm Selenium metabolism/Selenoproteins

Endochondral Ossification

Oxidative Stress

Osteoblast

GPCRs, Class B Secretin-like

Osteoclast

TGF-beta Receptor Signaling Pathway
Irinotecan Pathway

$\begin{array}{ccccc}(\mathbf{r}) & (\mathbf{n}) & \text { total } & \% & \text { z Score } \\ 4 & 10 & 13 & 40,00 \% & 10,28 \\ 5 & 43 & 49 & 11,63 \% & 5,67 \\ 5 & 56 & 68 & 8,93 \% & 4,77 \\ 2 & 23 & 29 & 8,70 \% & 2,94 \\ 1 & 7 & 11 & 14,29 \% & 2,86 \\ 1 & 10 & 13 & 10,00 \% & 2,28 \\ 1 & 13 & 18 & 7,69 \% & 1,9 \\ 4 & 115 & 149 & 3,48 \% & 1,89\end{array}$

pathways (down)

Irinotecan Pathway

Selenium metabolism/Selenoproteins

Endochondral Ossification

Oxidative Stress

Osteoblast

GPCRs, Class B Secretin-like

$\begin{array}{ccccc}(\mathbf{r}) & (\mathbf{n}) & \text { total } & \% & \text { Z Score } \\ 4 & 10 & 13 & 40 & 10,28 \\ 5 & 43 & 49 & 11,6 & 5,67 \\ 5 & 56 & 68 & 8,9 & 4,77 \\ 2 & 23 & 29 & 8,7 & 2,94 \\ 1 & 7 & 11 & 14,3 & 2,86 \\ 1 & 10 & 13 & 10 & 2,28\end{array}$

pathways

Irinotecan Pathway

Endochondral Ossification

Oxidative Stress

Cytokines and Inflammatory Response

TGF Beta Signaling Pathway

Selenium metabolism/Selenoproteins

Osteoblast

Prostaglandin synthesis and regulation

Adipogenesis

Osteoclast

Complement activation, classical pathway

pathways (up)

Adipogenesis

Prostaglandin Synthesis and Regulation

TGF Beta Signaling Pathway

Cytokines and Inflammatory Response

Oxidative Stress

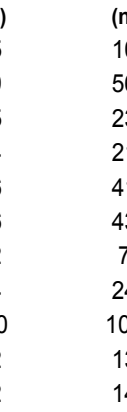

$\begin{array}{cccc}\text { (n) } & \text { total } & \% & \text { Z Score } \\ 10 & 13 & 50 & 7,59 \\ 56 & 68 & 16,1 & 4,79 \\ 23 & 29 & 21,7 & 4,47 \\ 21 & 25 & 19,1 & 3,63 \\ 41 & 52 & 14,6 & 3,61 \\ 43 & 49 & 14 & 3,46 \\ 7 & 11 & 28,6 & 3,4 \\ 24 & 31 & 16,7 & 3,27 \\ 108 & 132 & 9,3 & 2,97 \\ 13 & 18 & 15,4 & 2,16 \\ 14 & 16 & 14,3 & 2,03\end{array}$

$\begin{array}{ccccc}(\mathbf{r}) & (\mathbf{n}) & \text { total } & \% & \text { Z Score } \\ 10 & 108 & 132 & 9,3 & 4,48 \\ 4 & 24 & 31 & 16,7 & 4,38 \\ 5 & 41 & 52 & 12,2 & 3,92 \\ 3 & 21 & 25 & 14,3 & 3,4 \\ 3 & 23 & 29 & 13 & 3,19\end{array}$

Figure S7. Fold change increases during transit amplification

(A) Pie charts of all reporters fulfilling $\mathrm{p}<0.05$, FC $>1.1$ up or down, expression $>^{2} \log (100)$ in either group, for each time point in the experiment. (B) The table indicates for each time point: ${ }^{a}$ the number of reporters reaching the expression limit of ${ }^{2} \log (100)$ in either group; ${ }^{b}$ the number fulfilling significance $(p<0.05)$, FC > 1.1 (respectively 1.2) up or down, and expression above limit; ${ }^{c}$ the percentage of the latter versus the total number of expressed reporters. The figure is a graphical representation of the values in columns ${ }^{c}$. (C) Heatmap of the logratios of all reporters fulfilling $p<0.05, F C>2$ (left) or FC $<0.5$ (right), expression $>{ }^{2} \log (100)$ in either group, at 1 or 6 days pid. Euclidean distance and complete linkage hierarchical clustering were used to cluster and reorder the reporters. (D) Profile analysis of the most extremely de-regulated genes identified in expression arrays (up or down). (E) Biological pathways that are overrepresented based on Pathvisio analysis using as criteria: $p<0.05$ WT vs. Bmi1-KD, FC $>1.2$ up or down WT vs. Bmi1-KD, and average group expression ${ }^{2} \log (100)$ in either group, at 1 or 6 days pid, as compared to all reporters on the array for which the Affymetrix ID (or Unigene ID) could be mapped to a pathway (F) Biological pathways that are overrepresented based on Pathvisio analysis using as criteria: $p<0.05$ WT vs. Bmi1-KD, FC $>1.2$ up or down WT vs. Bmi1-KD, and average group expression ${ }^{2} \log (100)$ in either group, at 1 or 6 days pid, as compared to all reporters on the array for which the Affymetrix ID (or Unigene ID) could be mapped to a pathway. 
$\mathbf{A}$

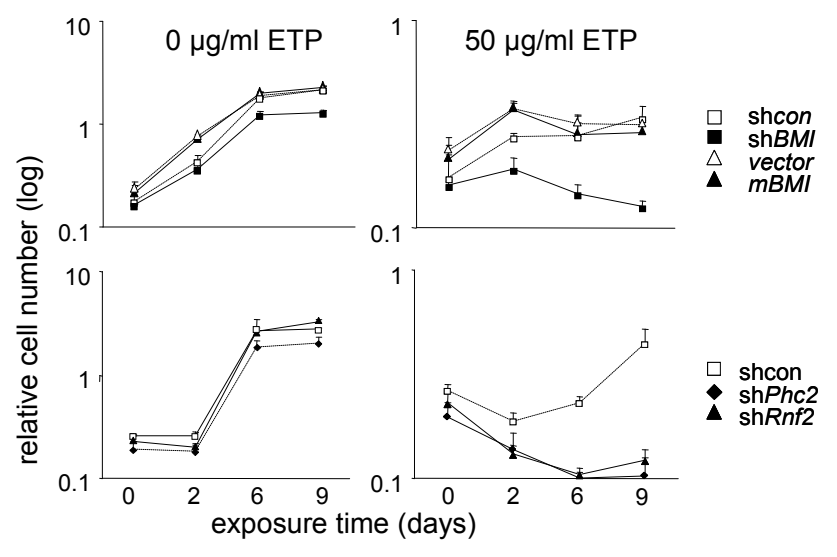

B
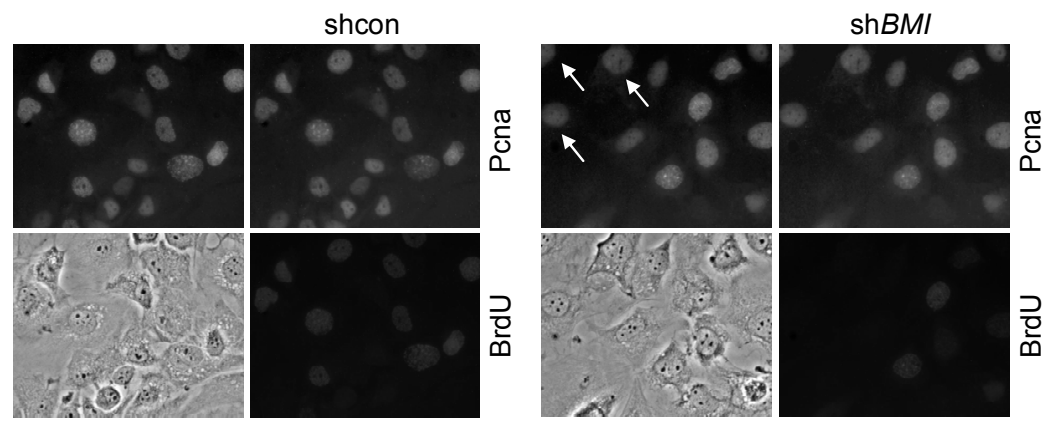

C

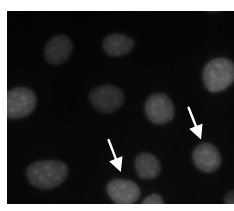

shcon
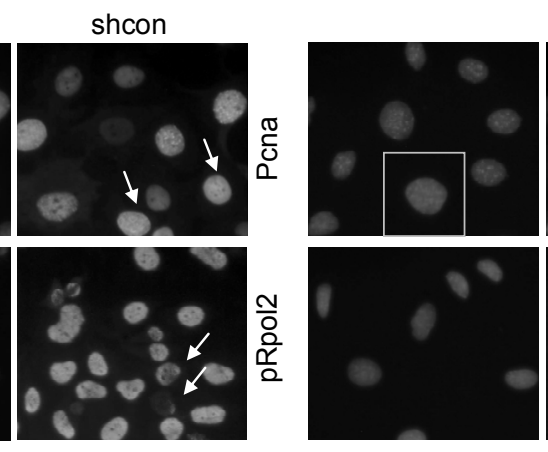

shBMI

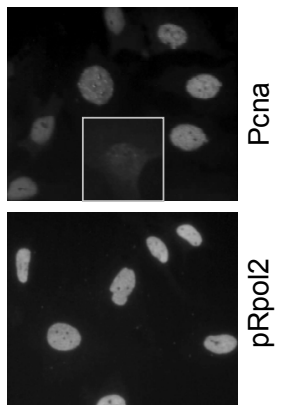

Figure S8. PRC1-LOF cultures are more sensitive to replication stress

(A) ATDC5 cells stably expressing shControl, shBmi1, shPhc2, shRnf2, vector or overexpress mBmi1 are exposed to 0 or $50 \mu \mathrm{g} / \mathrm{ml}$ etoposide for 1 hour, washed and allowed to recover for 9 days. Proliferation determined by Crystal violet incorporation with subsequent extraction and spectrophotometric analysis. (B,C) Co-staining for Pcna and BrdU or Pcna and pRNA polymerase II in ATDC5 shControl or shBmi1 at 2 days pid. 
Chapter 5

Supplemental Table 2

Primers use for qRT rtPCR

$\begin{array}{lll}\text { Cyclophillin } & \text { Forward } & \text { CAAATGCTGGACCAAACACAA } \\ & \text { Reverse } & \text { TTCACCTTCCCAAAGACCACAT } \\ \text { Bmi1 } & \text { Forward } & \text { TGGAGAAGAATGGCCACT } \\ \text { Collagen 2a1 } & \text { Reverse } & \text { GCAAGTTGGCCGAACTCTGT } \\ & \text { Forward } & \text { CAAGAACCCTGCTCGCACTT } \\ \text { Aggrecan } & \text { Reverse } & \text { CCGCTCTTCCACTCGGG } \\ & \text { Forward } & \text { CATGAGAGAGGCAATGGAA } \\ \text { Collagen 10a1 } & \text { Reverse } & \text { TGATCTCGTAGCGATCTTTCTTCT } \\ & \text { Forward } & \text { CATGCCTGATGGCTTCATAAA } \\ & \text { Reverse } & \text { AAGCAGACACGGGCATACCT }\end{array}$




\section{Chapter 6}

\section{Proliferative Capacity Defines Sensitivity to Valproic Acid During Chondrogenesis.}

Frank Spaapen ${ }^{1}$, Vivian Dahlmans ${ }^{1}$, Andy Creemers ${ }^{2}$, Pieter Emans ${ }^{2}$, Tim Welting $^{2}$, J. Willem Voncken ${ }^{1 *}$.

1 Department of Molecular Genetics, GROW - School for Oncology and Developmental Biology,

Maastricht University, Maastricht, The Netherlands;

2 Department of Orthopaedics, GROW - School for Oncology and Developmental Biology,

Maastricht University, Maastricht, The Netherlands

* Corresponding author: J.W. Voncken (w.voncken@gen.unimaas.nl) 


\begin{abstract}
Valproic acid (VPA) is a drug used to treat epilepsy, schizophrenia and other neurological conditions. VPA administration during pregnancy can have severe adverse effects on the unborn child, including neural tube defects and skeletal malformations. During development stem cells undergo lineage commitment and ultimately adopt terminally differentiated states. Epigenetic regulation, i.e. chromatin remodeling, controls gene-environment interactions during development. Although recently VPA was identified as an epigenetic drug, the mechanism(s) of action for VPA remain unclear. VPA has been studied in the context of chondrogenesis, yet published studies report conflicting results. In this study we use the widely used ATDC5 model to study geneenvironment interactions in the context of chondrogenesis and to elucidate effects of VPA treatment on chondrogenic differentiation. We find that VPA treatment severely affects chondrogenesis: ATDC5 fail to induce the transcriptional reprogramming required for chondrogenesis. Further, VPA affects differentiation induced hyperproliferation in a TP53 dependent manner. Relevantly, VPA treatment in ATDC5 cells causes replication stress, DNA damage response and results in a senescence-like response. We show that epigenomic remodeling is essential for proper induction of differentiation programs. Our findings support the notion that VPA treatment can be useful to sensitize rapidly proliferating cells to replication stress, providing therapeutic applications for cancer treatment.
\end{abstract}




\section{Introduction}

Valproic acid (VPA) is a pharmacological compound widely used to effectively treat convulsive disorders, migraine, bipolar disorders and schizophrenia. VPA use during pregnancy, however, causes severe developmental abnormalities, both in humans and various laboratory animals: teratogenic effects in mice include neural tube defects, as anencephaly and spina bifida, and skeletal malformations, predominantly along the anteroposterior (AP) axis ${ }^{1-6}$. The outcome of VPA treatment is cell type dependent: rat embryonic neural progenitor cultures (cerebral cortex or hippocampus), show enhanced neurogenesis, and reduced proliferation, accompanied by increased promoter acetylation and expression of neural transcription factors ${ }^{7,8}$. VPA enhances hepatic and osteogenic differentiation in bone marrow derived and adipose tissue derived stromal stem cell cultures, respectively ${ }^{9,10}$. Conversely, in various cancer cell types VPA is toxic and leads to senescence and cell death by apoptosis or other mechanisms ${ }^{11-14}$. VPA toxicity in embryonic stem cells is manifested in reduced cell viability and a potent inhibition of mesodermal and endodermal development ${ }^{15}$. Also, at non-cytotoxic concentrations VPA causes abnormal cartilage matrix gene expression in the lumbar spine in chick embryos ${ }^{1}$. Although the molecular bases for its effects are poorly understood, experimental studies demonstrated that VPA treatment affects multiple regulatory pathways: Akt activation and consequent glycogen synthase kinase $3 \beta$ inhibition have been reported ${ }^{16}$, besides stimulatory effect on the GABA and NMDA neurotransmitter receptors ${ }^{17}$.

Recently, VPA was shown to directly inhibit histone deacetylases (HDACs) ${ }^{18}$. HDACs play an important role in DNA templated processes, such as transcription and replication, through epigenetic remodeling of chromatin. Epigenetic regulation of chromatin structure is widely studied in the context of cancer and development. In pluripotent embryonic stem cells (ESCs) key developmental regulator genes, including Hox genes, display large stretches of repressionassociated $\mathrm{H} 3 \mathrm{~K} 27 \mathrm{me} 3$ and activating $\mathrm{H} 3 \mathrm{~K} 4 \mathrm{me} 3$ at their promoters. This seemingly conflicting combination of modifications was termed 'bivalency' ${ }^{19}$. Differentiation of ESCs is accompanied by remodeling of the epigenome, to facilitate activation of appropriate lineage specific gene expression, and epigenetic silencing of non-lineage specific genes ${ }^{19}$. Not surprisingly, many mutant epigenetic regulator genes display developmental defects. Polycomb group (PcG) and Trithorax group (trxG) mutant mice, associated with $\mathrm{H} 3 \mathrm{~K} 27 \mathrm{me} 3$ and $\mathrm{H} 3 \mathrm{~K} 4 \mathrm{me} 3$ marks, respectively, are responsible for malformations along the antero-posterior (AP) axis. Skeletal phenotypes correlate with deregulation of expression boundaries within HOX gene clusters, wellknown PcG and trxG target genes ${ }^{20-22}$. Combined, this demonstrates how proper epigenetic regulation is pivotal to proper cellular differentiation.

Surprisingly little is known about the molecular mechanisms by which VPA affects endochondral ossification (formation of an ossified skeleton from a cartilage scaffold). VPA treatment in utero was reported to affect expression of Hox genes and PcG genes in whole mouse embryos ${ }^{3,4,23}$. The 


\section{Chapter 6}

mature ossified skeleton of mammals arises from a cartilage scaffold. However, direct effects of VPA on chondrogenesis remain poorly understood. The present study therefore aimed to further elucidate effects of VPA in development, with a focus on epigenetic deregulation during chondrogenesis. ATDC5 cells are a widely used model to study chondrogenesis. A remarkable feature of ATDC5 cell differentiation is an early, transient hyperproliferative burst upon induction of differentiation. This phase reflects the transit amplification that mesenchymal progenitor cells undergo during development. We assessed the effects of VPA on ATDC5 chondrogenic differentiation and proliferation. Briefly, we find that VPA specifically impedes proliferation of transit amplifying cells in a Trp53(P53)-dependent manner. In addition, VPA completely abolishes chondrogenic marker gene expression, demonstrating a direct and dramatic effect on chondrogenesis. We here discuss these effects in the context of altered epigenetic control.

\section{Materials \& Methods}

\section{Cell culture and VPA treatment}

ATDC5 cells were cultured at $37{ }^{\circ} \mathrm{C}, 5 \% \mathrm{CO} 2,100 \%$ humidity in DMEM/ F-12 supplemented with $5 \%$ fetal calf serum (FCS), antibiotics (100 units/ml penicillin and $100 \mu \mathrm{g} / \mathrm{ml}$ streptomycin), $200 \mathrm{mM}$ L-glutamine on tissue culture plates (Greiner Bio-One). For differentiation experiments cells were seeded at 6400 cells $/ \mathrm{cm}^{2}$ and were allowed to attach overnight. Growth medium was changed for differentiation medium, which includes ITS $\left(10 \mu \mathrm{g} / \mathrm{ml}\right.$ insulin, $10 \mu \mathrm{g} / \mathrm{ml}$ transferrin and $3 \times 10^{-8} \mathrm{M}$ sodium selenite). Differentiation medium was replaced every two days. VPA (Sigma, P4543) was prepared freshly and supplemented to differentiation medium to pharmacologically relevant end concentrations of $0.3,1$ and $3 \mathrm{mM}$ (FDA).

\section{Retroviral infections}

Ecotropic retroviral supernatants were produced by transfection of phoenix cells with calciumphosphate precipitation; 24-48 hours post-transfection, the supernatant was harvested, filtered and used for infection of ATDC5 cells in presence of $4 \mu \mathrm{g} / \mathrm{ml}$ polybrene. Cells were incubated for 12 hours and then allowed to recover for 24 hours on fresh medium before selection pressure was applied. Infected cells were selected with puromycin $(8 \mu \mathrm{g} / \mathrm{ml})$ for 72 hours, before experiments were initiated. Short-hairpin (sh)RNA vector used for RNA interference-mediated knock-down of murine Trp53 was described elsewhere ${ }^{24}$. Short hairpin sequence targeting shGFP was used as reference sh sequence ${ }^{25}$.

\section{Chrystal violet and Alcian blue stainings}

ATDC5 cells were plated in 12-multiwell plates (Greiner Bio-one). At each time point cells were washed twice with phosphate-buffered saline, and fixed for 10 minutes with $3.7 \%$ formaldehyde at room temperature. Next, cells were rinsed 5 times with demiwater. Cells were stained with $0.1 \% \mathrm{X}$ - 
Proliferative Capacity Defines Sensitivity to VPA During Chondrogenesis tal violet or $0.1 \%$ Alcian blue for 30 minutes or overnight, respectively, and washed 5 times with demiwater. X-tal was extracted with $10 \%$ acetic acid and Alcian blue with guanidine/HCL (6M) and absorbance was measured spectrophotometrically at $590 \mathrm{~nm}$ or $645 \mathrm{~nm}$, respectively (Benchmark, Biorad).

\section{Senescence-associated $\beta$-galactosidase (SA-bGal) assay}

Staining was performed according to protocol described by Dimri et al. [26]. Shortly, cells were washed in PBS, fixed for 3-5 min (room temperature) in 2\% Formaldehyde $/ 0.2 \%$ glutaraldehyde, washed, and incubated at $37^{\circ} \mathrm{C}$ (ambient $\mathrm{CO}_{2}$ ) with fresh senescence associated (3-Gal (SA-,3-Gal) stain solution: $1 \mathrm{mg}$ of 5-bromo-4-chloro-3-indolyl P3-Dgalactoside $(\mathrm{X}-\mathrm{Gal}$ ) per $\mathrm{ml}$ (stock $=20 \mathrm{mg}$ of dimethylformamide per $\mathrm{ml}$ ) $/ 40 \mathrm{mM}$ citric acid/sodium phosphate, $\mathrm{pH}$ 6.0/5 $\mathrm{mM}$ potassium ferrocyanide/5 $\mathrm{mM}$ potassium ferricyanide/150 mM NaCl/2 mM MgCl2. SA-bGal activity was measured after 12-16 hours. Staining at $\mathrm{pH} 4.0$ was used as negative control; this detects lysosomal galactosidase activity.

\section{RNA isolation and cDNA synthesis}

Cells were suspended in Tri-reagent, and RNA was extracted according to the protocol of the manufacturer (Sigma). Quantity and purity of the RNA were determined by 260/280 nm absorbance measurements. cDNA was synthesized using iScript ${ }^{\mathrm{TM}} \mathrm{CDNA}$ synthesis kit (Biorad). Primers used are listed in supplemental Table 1.

\section{Western analysis}

Cells were lysed in RIPA buffer containing $5 \mathrm{mM}$ Benzamidine, $5 \mu \mathrm{g} / \mathrm{ml}$ Antipain, $5 \mu \mathrm{g} / \mathrm{ml}$ Leupeptin, $5 \mathrm{\mu g} / \mathrm{ml}$ Aprotinin, $1 \mathrm{mM}$ Sodium Vanadate, $10 \mathrm{mM}$ Sodium Fluoride, $10 \mathrm{mM}$ Pyrophosphate, $10 \mathrm{mM}$-glycerophosphate, $0.5 \mathrm{mM}$ DTT and $1 \mathrm{mM}$ PMSF. Lysates were subjected to 3 freeze-thaw cycles in liquid nitrogen, and to 3 cycles of sonication. After centrifugation for 10 minutes at $13.2 \mathrm{krpm}$, protein concentration was determined using a BCA protein assay kit (Pierce). Equal amounts of protein were boiled in Laemmli buffer and loaded on 9-15\% polyacrylamide gels. Proteins were transferred to polyvinylidene fluoride (PVDF) membranes. After blocking with $5 \%$ non-fat dry milk in PBS containing $0.1 \%$ Tween-20, membranes were incubated $\mathrm{o} / \mathrm{n}$ at $4^{\circ} \mathrm{C}$ with the following antibodies: anti $\beta$-actin Mab (C4, 691001, MP Biomedicals), anti Ezh2 Mab (BD43; coutesy D. Pasin Copenhagen, Denmark), anti Bmi1 Mab (F6; Courtesy M. van Lohuizen; Amsterdam, The Netherlands), anti H3K9Ac Poab (\#06599, Upstate Biotechnology) and anti H4K12Ac Poab (ab1761, Abcam). After extensive washing, membranes were incubated with corresponding horseradish peroxidase conjugated secondary antibodies for $1 \mathrm{~h}$ at room temperature. Signals were detected using enhanced chemoluminescence. 


\section{Immunofluorescence}

Briefly, cells were washed and fixed for $10-15$ minutes in $100 \%$ methanol at $-20^{\circ} \mathrm{C}$ and stored at $4^{\circ} \mathrm{C}$ in $70 \%$ ethanol or used directly for immuno-histochemistry. Cells were permeabilized for $5-10$ minutes in $0.2 \&$ triton- $X$ in PBS. Primary antisera were incubated for 1.5 hours at $37^{\circ} \mathrm{C}, 4-5 \mathrm{x}$ washed in $0.02 \%$ triton-X/PBS and fixed in $2 \%$ formaldehyde/PBS for 10 minutes at room temperature. Cells were washed with PBS and were than incubated with the primary antisera for 1.5 hours at $37^{\circ} \mathrm{C}, 4-5 \mathrm{x}$ washed in $0.02 \%$ triton-X/PBS, and incubated with secondary fluorescently labeled antibodies. All antisera were diluted and incubated in $0.02 \%$ triton- $X$ in PBS. Nuclei were counterstained with 4'-6-Diamidino-2-phenylindole (DAPI) and washed in $0.02 \%$ triton-X

/PBS. The last wash step was in PBS, upon which cells were dehydrated: 1 minute in $70 \%$ ethanol, 2x 1 minute in 100\% ethanol and air-dried. Cells were mounted in Vectashield (Vector Laboratories, Inc. Burlingame, CA) and analyzed using a NIKON TE200 Eclipse fluorescence microscope and photographed using a NIKON DXM1200 digital camare in combination with NIS Elements 3.0 Imaging software. The following antibodied were used: anti $\mathrm{YH} 2 \mathrm{~A} . \mathrm{X}$ Mab JBW301 (Upstate), anti p-Kap1S824 Poab (A300-767A, Bethyl laboratories) and anti H3K9me3 \#07-442 (Upstate).

\section{Cell cycle profiles}

Cells were fixed in cold methanol overnight at $-20^{\circ} \mathrm{C}$, washed and resuspended in PBS containing $100 \mu \mathrm{g} / \mathrm{ml}$ RNAse and $20 \mu \mathrm{g} / \mathrm{ml}$ PI for 2-3 hours at RT in dark environment, immediately prior to FACS analysis. Analysis was performed using a Becton Dickinson FACS machine with CellQuest software.

\section{Results}

\section{VPA inhibits chondrogenic differentiation}

To examine how VPA affects chondrogenesis ATDC5 cells were differentiated in the presence of neuropharmacologically relevant concentrations of VPA (range: 0.3, 1, 3.0 mM; therapeutic plasma concentrations: $0.3 \mathrm{mM}-1 \mathrm{mM}$ (FDA)). Starting cultures with low cell density were exposed to a combination of chondrogenic factors (Insulin, Transferrin, Selenite; ITS) and followed for 2 to 3 weeks. Chondrogenic differentiation was assessed by multiple parameters: chondrogenic marker gene expression, progenitor cell proliferation and alterations in morphological appearance. Under the experimental conditions applied in this study, VPA robustly inhibits differentiation even at the lowest concentration, as measured by realtime rtPCR of chondrogenic markers aggrecan (AGC), collagen type II (COL2A1) and collagen type X (COL10A1) after 10 and 15 days of differentiation 
(Figure 1A-C). Alcian blue staining for cartilage glycosaminoglycans confirmed the differential effects of VPA on low and high density ATDC5 differentiation (see below; Figure 3B).

Differentiating ATDC5 monolayers undergo a phase of hyperproliferation, and form threedimensional cartilage nodules [27]. VPA inhibits hyperproliferation during ATDC5 chondrogenesis in a dose dependent manner (Figure 1D) Consistent with altered cell
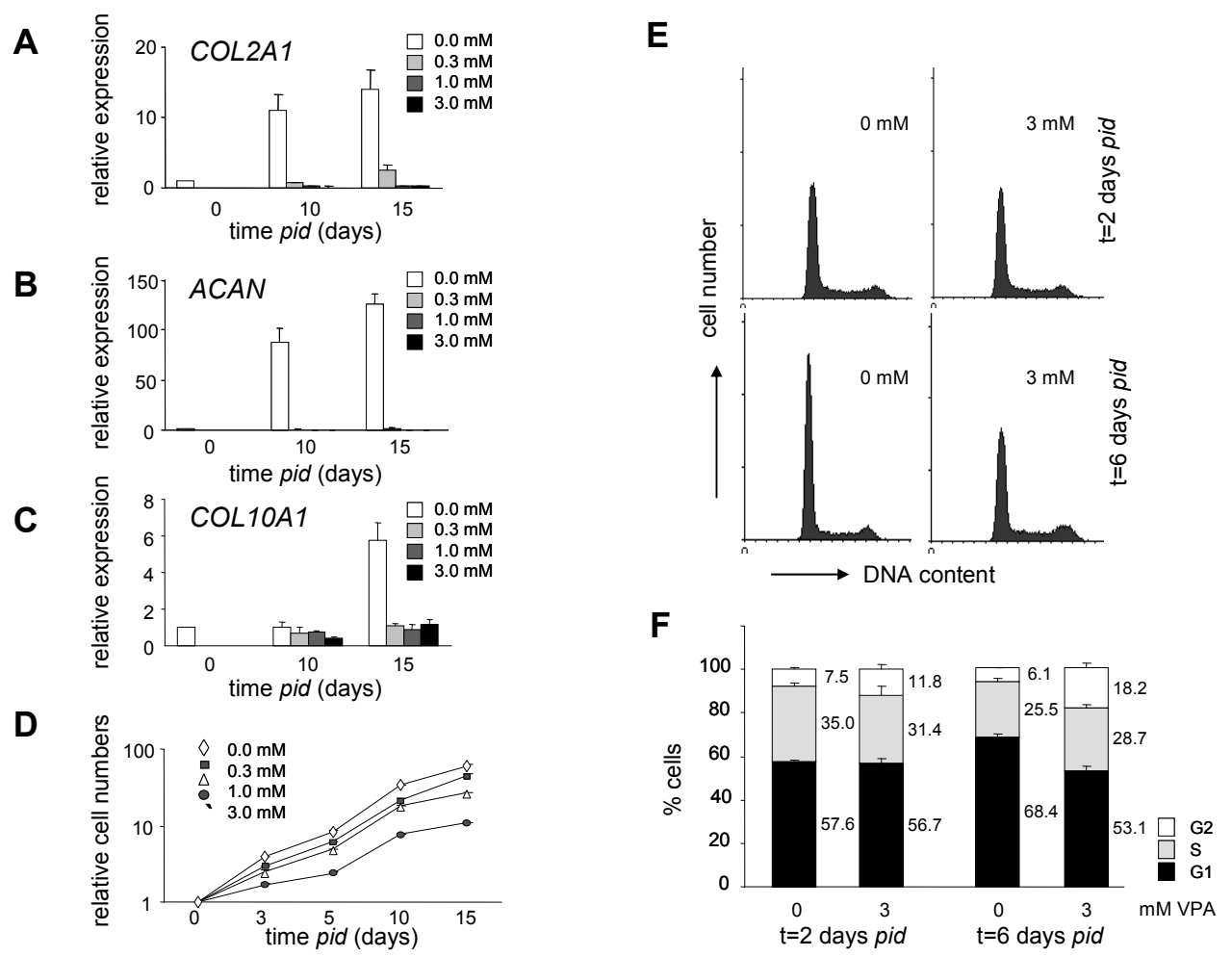

Figure 1. VPA blocks ATDC5 differentiation and proliferation. (A, B, C) qRT rtPCR analysis of chondrogenic markers of VPA treated ATDC5 cells. (D) VPA inhibits proliferation during ATDC5 differentiation. (E) Cell cycle profiles of VPA treated ATDC5 cells at 2 and 6 days of differentiation. $(F)$ Quantification of figure 1E.

cycle kinetics, DNA profiling showed an accumulation of tetraploid cells upon treatment with VPA: at $3.0 \mathrm{mM}$ VPA ATDC5 show an approximately 10-15\% increase in the G2/M peak (Figure 1E,F), mainly at the expense of diploid cells. In parallel with a dramatic decline in cell proliferation, Combined these results demonstrate that VPA blocks chondrogenic transcriptional reprogramming as well as differentiation induced hyperproliferation.

\section{VPA induces senescence in differentiating chondrocytes.}

VPA treatment was previously shown to induce cellular senescence ${ }^{13}$, a cellular phenotype which is associated with irreversible replicative arrest ${ }^{28}$. Senescent cultures often display a large-flat cell morphology accompanied by senescence related marker gene expression profiles. To determine 


\section{Chapter 6}

the adverse effect of VPA on cell cycling, we studied cell morphology. Both ATDC5 and primary human chondrocytes display the typical 'large flat cell' morphology upon exposure to VPA (Figure $2 A)$. Indeed, in good agreement with these morphological changes, staining for senescenceassociated- $\beta$-galactosidase (SA- $\beta$ Gal/Glb1) activity, a marker closely associated with replicative senescence, confirms a dose-dependent induction of senescence by VPA (see below; Figure 3C)). Expression profiling of senescence-associated marker genes further corroborated the senescence phenotype: GLB1 expression is upregulated in response to VPA throughout differentiation compared to control cells (Figure 2B). Chop (Ddit3/Gadd153), a protein involved in the unfolded protein response (UPR) and DNA damage responses, both of which are associated with senescence [29-33], is dramatically upregulated upon treatment with VPA (Figure 2C). Recently, elevated interleukin 6 (IL6) expression was established as a robust marker for senescenceassociated secretory phenotype (SASP; ${ }^{34,35}$ ). In keeping with this, we find that expression of IL6 was dose-dependently increased in differentiating ATDC5 cells upon treatment with VPA (Figure 2D). Finally, also the cell cycle inhibitor p19 arf was dose-dependently upregulated in VPA stimulated ATDC5 cells (Figure 2E), whereas $\mathrm{p} 16^{\mathrm{ink} 4 a}$, expressed from the same locus and associated with senescence, did not respond to VPA treatment (FS, JWV; unpublished data). Immunofluorescent analysis of VPA exposed cultures reveals phosphorylated histone H2A.X ( $\mathrm{YH} 2 \mathrm{~A} . \mathrm{X})$ and phosphorylated Kap1, both markers of early DNA damage responses (Figure 2F,G). Taken together, our findings convincingly show that VPA induces morphological changes, transcriptional reprogramming and DNA damage signaling associated with a senescence-like response during chondrogenesis.

A

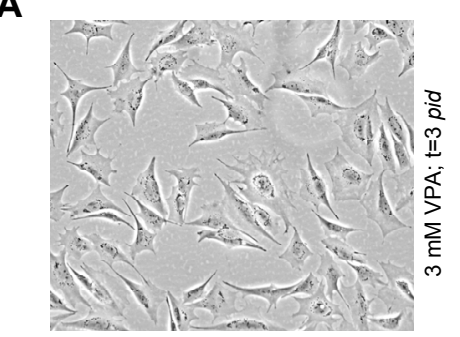

B

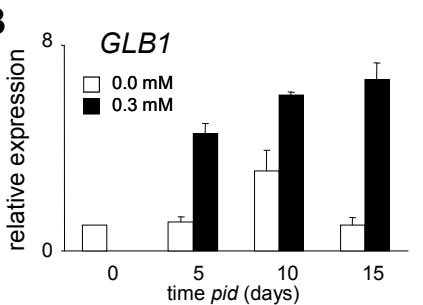

C

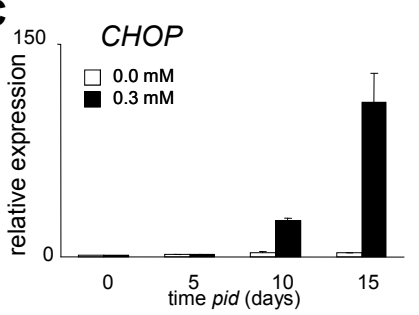

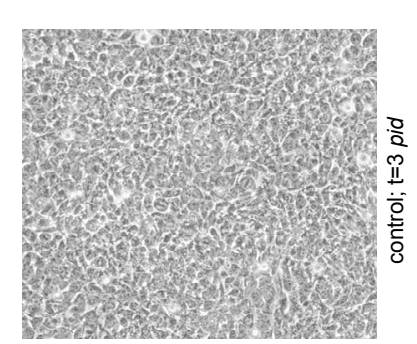

D

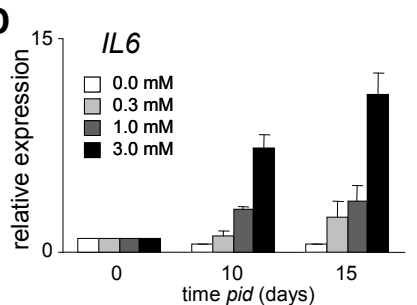

E

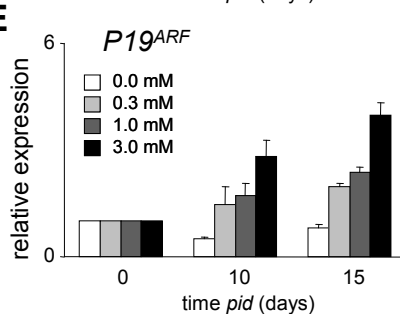

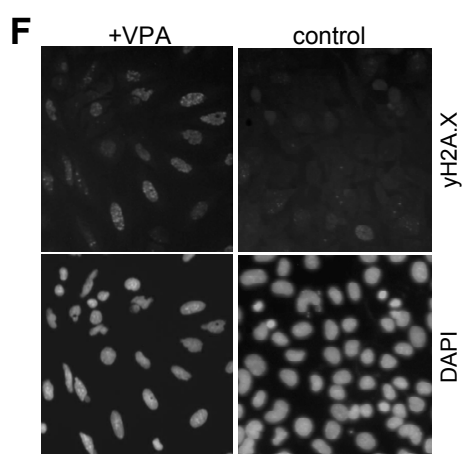

$\mathrm{t}=2$ pid (days)

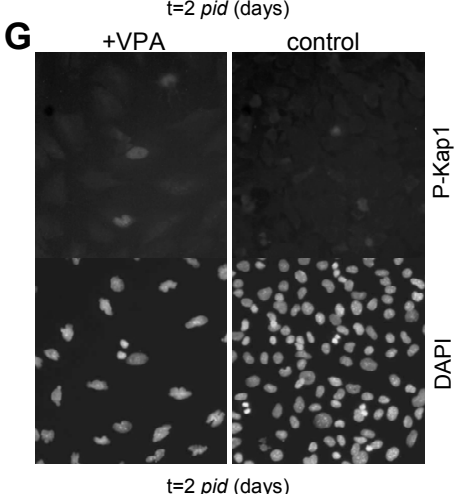

$\mathrm{t}=2$ pid (days) 
Figure 2. VPA induces senescence in differentiating chondrocytes.

(A) VPA induces a proliferative block and large flat cell phenotype. (B-E) qRT rtPCR analysis of genes associated with senescence in VPA treated chondrocytes. $(F, G)$ Chondrocytes treated with VPA $(3 \mathrm{mM})$ accumulate DNA damage, measure by $\mathrm{YH} 2 \mathrm{Ax}(\mathrm{F})$ and $\mathrm{p}-\mathrm{Kap} 1(\mathrm{G})$.

\section{VPA induced inhibition proliferation, but not differentiation, is dependent on TP53}

We next investigated the pathways involved in VPA-induced senescence. In the context of oncogene-induced senescence, activation of a DNA damage responses (DDR) is sufficient to induce senescence, however, inactivation of the DDR, e.g. by ablation of Trp53 or ATM, bypasses proliferative arrest ${ }^{32,33}$. The cell cycle regulators $\operatorname{Trp} 53$ and $\mathrm{p} 19^{\mathrm{ARF}}$ are both causally involved in replicative senescence; P19 ARF blocks MDM2-induced proteolytic degradation of Trp53 ${ }^{36,37}$. To determine whether the effects of VPA on chondrogenic differentiation are dependent on Trp53, we stably expressed a short hairpin (sh)RNA which targets Trp53 mRNA for degradation 24,38. Remarkably, ablation of Trp53 reproducibly enhances proliferation in control cultures (Figure 3A top panel; FS, JWV unpublished data) and induction of chondrogenesis allows for higher production of glycosaminoglycans in absensce of P53 (Figure 3B). Loss of Trp53 function (LOF) rescues proliferation in differentiating ATDC5 cells at low VPA concentration range to a proliferative rate observed in normal chondrocytes (0.3 and $1.0 \mathrm{mM}$ VPA; Figure 3A). VPA was reported to induce abnormal nuclear morphology in cancer cells ${ }^{39}$. We find that VPA also dramatically changes nuclear morphology in chondrogenic cultures at high concentration: nuclei acquire extremely irregular multi-lobed shapes irrespective of Trp53 (Figure 3C; lower panels). We recently discovered that SAbGal is induced as part of normal chondrogenesis (FS, JWV in preparation); as such the effect of VPA on SAbGal induction can only be studied under conditions in which VPA induces an acute proliferative arrest (preceding hyperproliferation). 3 mM VPA activates the typical bGal staining observed in senescent cells ${ }^{26}$. Remarkably, when stained for SAbGal, we find that Trp53-LOF prevents SAbGal activation at the higher VPA concentrations (1.0 and $3.0 \mathrm{mM}$; Figure $3 \mathrm{D}$ ), suggesting that transcriptional activation of or signaling to SAbGal is dependent on Trp53.

Surprisingly, Trp53-LOF does not rescue the defective differentiation of VPA treated ATDC5 cells, as measured by Alcian Blue staining for cartilage matrix glycosaminoglycans (Figure 3B) and quantitative RT/rtPCR of chondrogenic markers (Figure 3E): expression of key chondrogenic regulator genes SOX9 and RUNX2 is differentiatally affected by Trp53 or p19 ${ }^{\text {ARF }}$-LOF: RUNX2 mRNA is clearly suppressed at 7-10 days pid, whereas SOX9 expression appears largely unaffected. Conversely, although Trp53 or p19 ARF -LOF leads to an exaggerated SOX9 and RUNX2 induction at 10 days pid (Figure $3 \mathrm{E}$ ), which correlates with the increased alcian blue staining at this timepont (Figure 3B). Overall, however, Trp53 or p19 $9^{\mathrm{ARF}}$-LOF do not rescue terminal chondrogenic capacity as judged by $A C A N, C O L 2 A$ and $C O L 10 A 1$ expression and glycosaminoglycan staining (Figure 3F,B). We conclude from this combined data that Trp53-dependent proliferative control is an important component of chondrogenic differentiation, but that chondrogenic transcriptome reprofiling is not controlled by Trp53. 


\section{VPA affects epigenetic modifiers and histone modifications}

To study possible effects of VPA on epigenetic regulation during chondrogenesis we examined expression on epigenetic regulators belonging to the Polycomb Group proteins in VPA exposed cultures. A recent study implicated reduced Polycomb Group gene expression in abnormal skeletogenesis of VPA-exposed fetuses in utero ${ }^{23}$. We therefore investigated the effect of VPA on expression of a number of relevant Polycomb genes. Polycomb repressive protein complexes (PRCs) harbor enzymes with intrinsic epigenetic activities, among which histone H3 lysine 27 methyl transferase activity

A
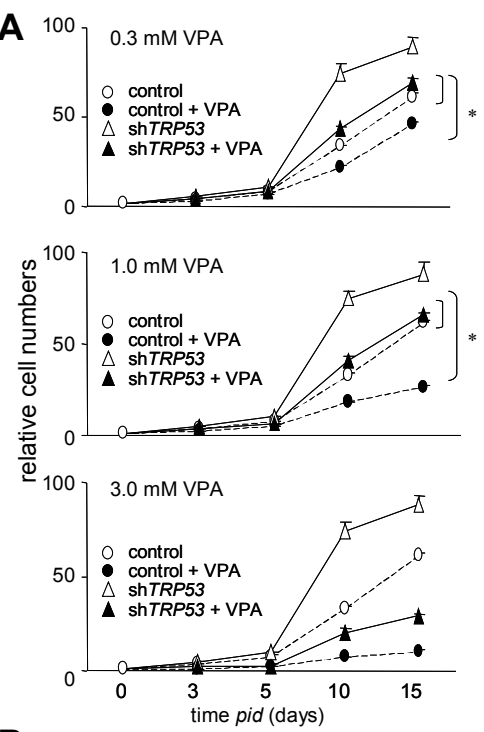

B

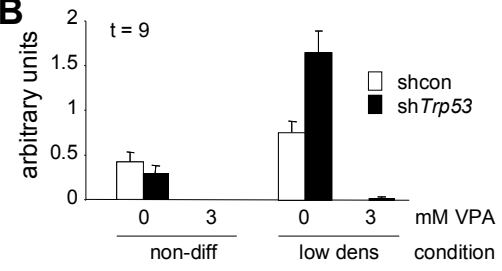

E

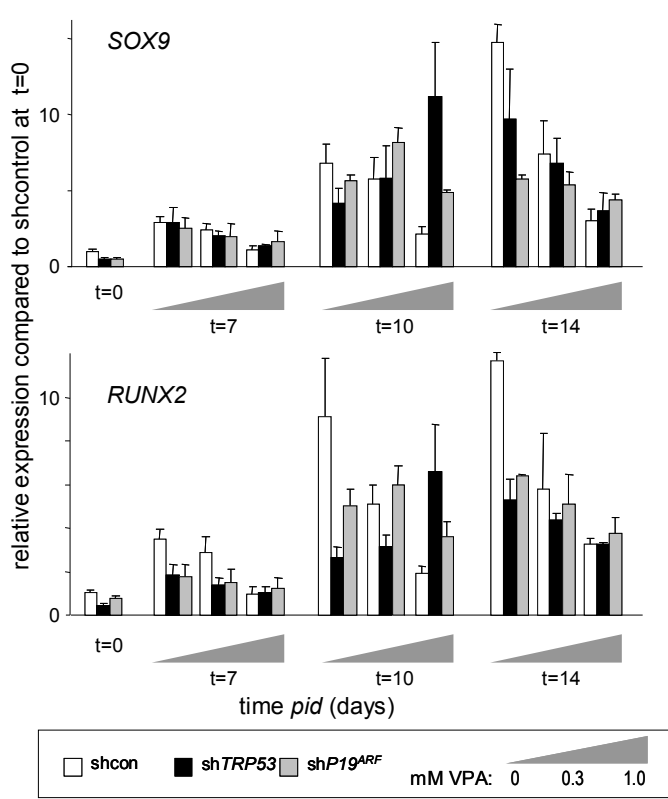

C
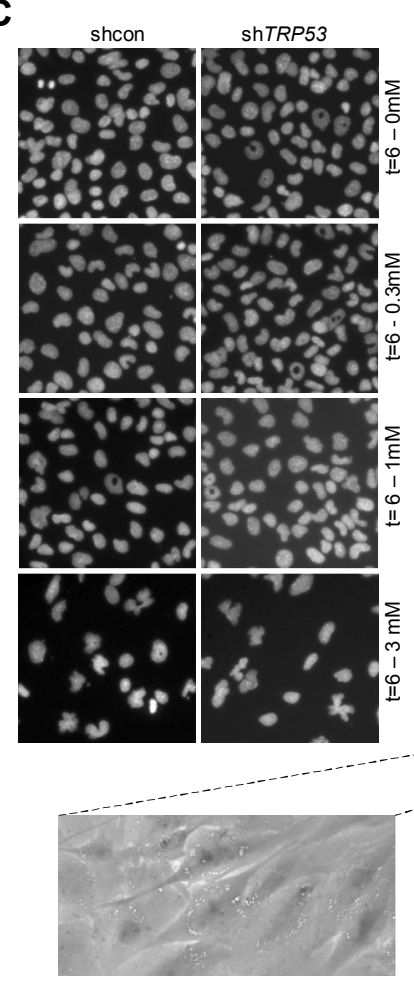

F
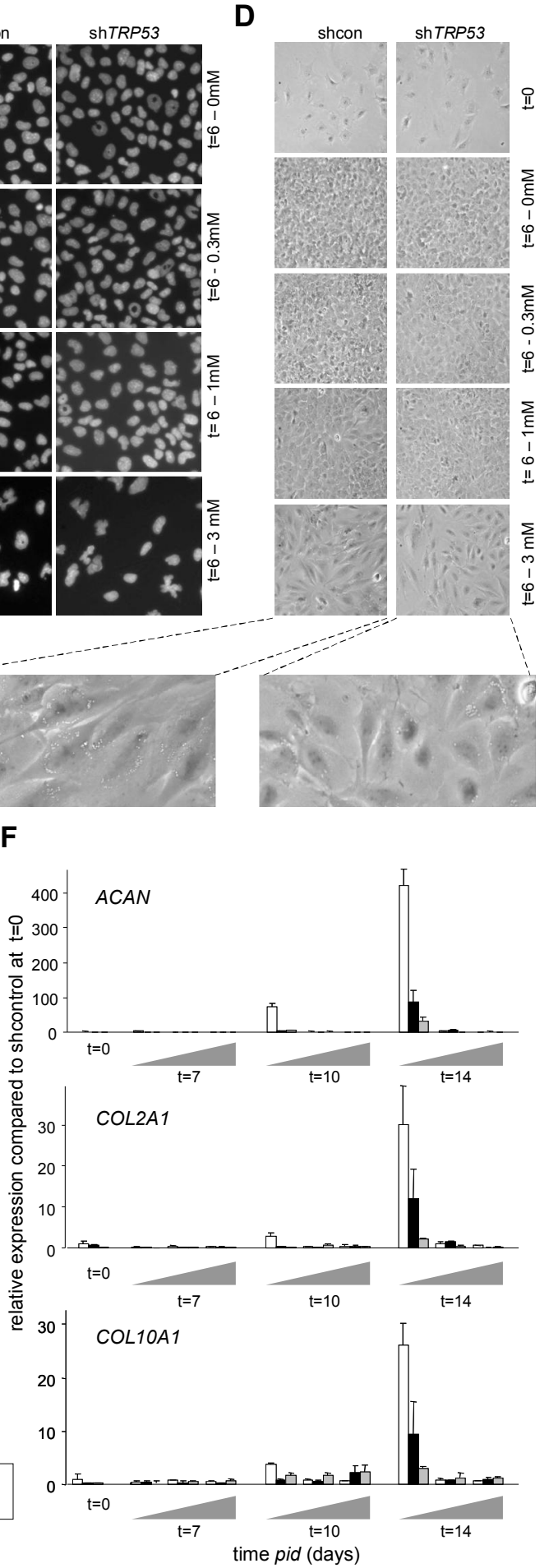
Figure 3. VPA induced inhibition proliferation, but not differentiation, is dependent on TP53.

(A) Expression of shTP53 rescues part of VPA induced proliferation block. (B) Quantification of alcian blue staining from ATDC5 cells stably expressing shControl and shTP53, in VPA treated $(3 \mathrm{mM})$ and non-treated differentiating chondrocytes. (C) Nuclear morphology (DAPI staining) of ATDC5 cells stably expressing shControl and shTP53 treated with VPA $(0,0.3,1$ and $3 \mathrm{mM})$ at day 6 of differentiation. (D) SAbGAL staining on shControl and shTP53 expressing ATDC5 cells treated with VPA $(0,0.3,1$ and $3 \mathrm{mM})$ at day 6 of differentiation. (E,F) qRT rtPCR analysis of chondrogenic markers of VPA treated $(0,0.3$ and $1 \mathrm{mM})$ ATDC5 cells expressing shControl, shTP53 or shP19 ${ }^{\mathrm{ARF}}$.

(EZH2), histone H2A lysine 119 ubiquityl E3 ligase activity (RNF2, BMI1, RING1). Expression analysis of these factors revealed apparent regulation of PRC gene expression through out chondrogenesis (Figure 4A, white bars), however, transcriptional deregulation in the context of VPA exposure failed to show significant reproducible changes in expression or consensus trends among PRC genes (Figure 4A, shaded bars). Of PRC genes examined, VPA only reduces BMI1 expression at 6 days pid, both at the mRNA and protein level (Figure 4A,B).
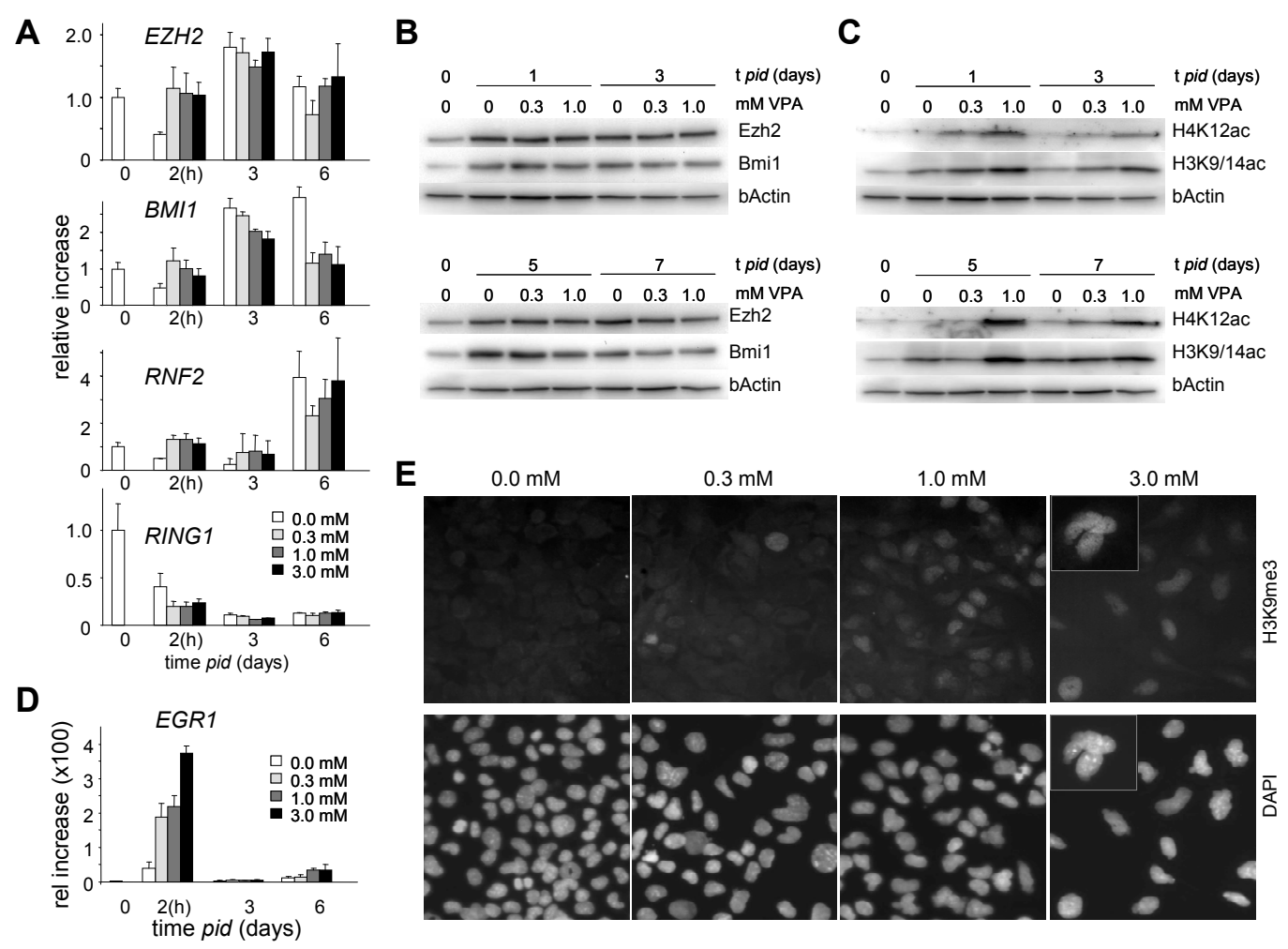

Figure 4. VPA affects epigenetic modifiers and histone modifications.

(A) qRT rtPCR analysis of epigenetic modifiers in ATDC5 treated with VPA $(0,0.3,1$ and $3 \mathrm{mM})$ at $\mathrm{T}=0,2 \mathrm{~h}, 3 \mathrm{~d}$, and $6 \mathrm{~d}$.

(B) ATDC5 cells were differentiated for $0,1,3,5$ and 7 days, treated with VPA (0, 0.3 and $1 \mathrm{mM})$ and analyzed for protein expression of epigenetic modifiers. (C) ATDC5 cells were differentiated for $0,1,3,5$ and 7 days, treated with VPA (0, 0.3 and $1 \mathrm{mM}$ ) and analyzed for expression of histone modifications. (D) qRT rtPCR analysis of Egr1 in ATDC5 with VPA (0, 0.3, 1 and $3 \mathrm{mM}$ ) at T=0, 2h, 3d, and 6d. (E) VPA dose-dependent increase in H3K9me3 levels in ATDC5 and DAPI nuclear staining 
To determine global changes in epigenetic marking as a result of VPA exposure, we analyzed covalent histone modifications throughout chondrogenesis in control cultures $(t=0)$ and compared these to cultures exposed to VPA. Covalent histone modifications were analyzed using specific antisera against acetylated lysine 9 and 14 on histone 3 (H3K9/14ac) or H4K12ac. We find a dose dependent hyperacetylation of histone $\mathrm{H3}$ and 4 in response to VPA (Figure 4C). This global hyperaceylation correlates well with substantially increased early chondrogenic expression of the immediate-early response gene EGR1, which was reported to recruit histone acetyl transferases (HAT; Figure 4D). Finally, H3K9 trimethylation, which is associated with cellular senescence (Lowe), is induced in a dose dependent manner by VPA (Figure 4E). Relevantly, nuclei with altered morphology display very high H3K9me3 levels (Figure 4E inset). Combined these data substantiate the importance of epigenetic modulation early in chondrogenesis and confirm a prominent role for VPA during the early stages of chondrogenic differentiation.

\section{Discussion}

The widely used anticonvulsant and mood-stabilizing drug VPA is known to cause brain damage and birth defects in the fetus. As the molecular mechanisms underlying VPA-induced AP-axis abnormalities remained incompletely understood, we investigated VPA in the context of chondrogenic differentiation. We report here that VPA treatment strongly inhibits chondrogenic differentiation and blocks progenitor proliferation during the hyper-proliferative phase in chondrogenic differentiation. Furthermore, we show that VPA induces a stress response that activates TRP53-dependent proliferative block during differentiation induced hyperreplication.

Chondrogenic differentiation is a multi-phasic process, which is controlled by a variety of transcription factors, growth and differentiation factors. During the earliest phase of chondrogenic differentiation the cells proliferate rapidly, which is critical for formation and extension of the cartilage scaffolds that ultimate will form bony elements of the skeleton. The Sox-family of transcription factors are key regulators of these early phases of differentiation. Eventually, the differentiating chondrocytes withdraw from the cell cycle and become hypertrophic. Toward terminal differentiation chondrocytes excrete a variety of extracellular matrix proteins (i.a. Col2A1; Col10A1; Acan) that will constitute the cartilage matrix. Hypertrophic chondrocytes excrete factors which initiate vascularization and attract osteoblasts. Eventually hypertrophic chondrocytes undergo apoptosis, are replaced by invading osteoblasts, which signals ossification of the cartilage matrix ${ }^{40}$.

Previous reports established axial skeletal transformations in mice exposed to VPA in utero ${ }^{4,23}$. Also in lumbar spines from chick embryos cultured ex vivo VPA was found to affect mRNA 
expression of collagen IX and Aggrecan, and VPA induced cytotoxicity in mesenchymal cells isolated from the chick spine in a dose-dependent manner ${ }^{1}$. Our studies which show a dramatic effect of VPA on chondrogenesis in ATDC5 are in good agreement with these findings. A recent study reported no effects of VPA on ATDC5 chondrogenic differentiation when differentiation was initiated in a high density culture ${ }^{41}$. These differences may be explained by differences in experimental procedure: protocols applied in the current study ${ }^{27}$, induce differentiation in ATDC5 cultures at low density, whereas the reported protocol involved pre-plating of cells and proliferation to confluency, and subsequent induction of ITS-mediated differentiation. The original ATDC5 differentiation protocols revealed a hyperproliferative burst and the subsequent formation of multilayered cartilage nodules ${ }^{27}$, thus allowing detailed analysis of both proliferation dynamics as well as transcriptome reprogramming. Preliminary comparative analysis of these two differentiation protocols shows that initiation of differentiation at high confluency does not lead to induction of hyperproliferation and formation of cartilage nodules (FS,TW,JWV unpublished data), most likely since cells have already ceased cycling at the start of differentiation. Together with our finding reported here, that chondrogenic hyperproliferation is controlled by Trp53, this further supports the notion that hyperproliferation is an essential element during chondrogenesis. Remarkably, Trp53LOF does not rescue the ability of VPA-exposed cells to differentiate. This points to a distinctive role for Trp53 in cell proliferation, rather than differentiation. Indeed, similar observations were made in zebrafish studies recently ${ }^{42}$. These collective observations underscore the notion that transcriptional reprogramming and proliferation are integrated, yet distinctively regulated processes during chondrogenesis.

In addition to apoptosis, senescence is increasingly recognized as a mechanism to protect cells from oncogenic transformation ${ }^{43}$. Ectopic expression of oncogenes, such as RAS and BRAF in normal diploid fibroblasts evokes oncogene induced senescence (OIS) ${ }^{32,44}$. Upon oncogenic activation these cells first undergo a short phase of hyperproliferation, after which replication is markedly reduced and senescence sets in. The origin of replicative senescence is viewed as an inability of cells to keep up with hyperproliferation, which results in DNA damage, stalled replication forks and, importantly, a replication stress-induced DNA damage response (DDR) which triggers DDR dependent OIS; indeed, ablation of DDR (ATM, ATR, Trp53) bypasses aspects of OIS ${ }^{29-33}$. Analogous to oncogenic activation of fibroblasts, ATDC5 differentiation includes a phase of rapid DNA replication. In keeping with activation of a senescence-like response by VPA, we find a number of senescence markers upregulated in VPA exposed cultures: the tumor suppressor p19 $19^{A R F}$ is progressively upregulated in VPA exposed chondrogenic cultures. Consistent with this, ATDC5 cells respond to VPA induced stress by undergoing Trp53-dependent proliferative arrest and senescence. P19ARF is known to stabilize Trp53 and early senescent-associated responses are known to involve both factors. In addition, elevation of Chop, also known as DNA damage inducible transcript-3 (Ddit3)/Gadd153, supports a role for DDR in VPA-induced senescence. Our findings are consistent with recent reports that VPA induces a senescent-like phenotype in 


\section{Chapter 6}

medulloblastomas ${ }^{11}$. In addition, several reports show that VPA induces a proliferative arrest in cancer cells of haematologic origin 12,45 , which may be associated with activation of autophagocytosis, a phenomenon known to be related to ERS and UPR ${ }^{30,44}$. The molecular basis of the VPA-induced DDR is not fully clear. VPA was shown to induce a variety of stresses, e.g. ER stress and production of reactive oxygen species (ROS) ${ }^{46,47}$. However, the mechanisms by which VPA treatment leads to ER stress or ROS production remain elusive. Consistent with the herein reported compromising effect of VPA on the ability of ATDC5 cells to hyper-replicate DNA, others have shown an enhanced sensitivity to the topoisomerase poisons ${ }^{48}$. Finally, we show that H3K9me3 is strongly induced upon VPA exposure, providing an additional link to senescence. Senescent tumor cells show accumulation of $\mathrm{H} 3 \mathrm{~K} 9 \mathrm{me} 3{ }^{49}$. Although the exact role of $\mathrm{H} 3 \mathrm{~K} 9 \mathrm{me} 3$ in OIS is still not clear ${ }^{50}$, a global increase of $\mathrm{H} 3 \mathrm{~K} 9 \mathrm{me} 3$ may be part of a cellular stress response (FS, PP, JWV unpublished data).

Despite lack of experimental evidence for a generally reduced Polycomb function, the effect of VPA on chondrogenesis unlikely affects Polycomb gene expression directly, it is possible that abnormal associated epigenetic activity, like blocked HDAC function, contributes to the skeletal phenotype ${ }^{1,4,51}$. The reduction of the PRC1 protein BMI1 upon VPA exposure is interesting, in that it is consistent with our recent observation that Bmi1 is a prime regulator of chondrogenesis (FS, JWV; in preparation). In addition to possible direct effects on chondrogenesis, Bmi1-LOF was reported to induce senescence ${ }^{52,53}$; as such the VPA-induced response could be in part explained by defective Bmi1 function.

We here report a concentration dependent hyperacetylation of histones $\mathrm{H} 3$ and $\mathrm{H} 4$ during the early phases of chondrogenesis in VPA-exposed cells. These observations are consistent with published reports on the effect of VPA on epigenome-wide histone acetylation in various cells ${ }^{18}$. It has been speculated that the HDAC inhibiting activity of VPA promotes hyperacetylation leaving chromatin in an open configuration and more susceptible to DNA damage during replication ${ }^{48}$. Interestingly, VPA induces a strongly amplified expression of EGR1, an immediate early gene we recently identified as a crucial factor for early epigenomic reprogramming in chondrogenesis (FS, JWV; in preparation). Egr1 recruits CPB/p300 HAT activity to promoters. It is conceivable that this exaggerated EGR1 activation is instrumental in the observed abnormal epigenomic responses. Relevantly, hyperacetylation has been connected to polyploidy, a phenomenon which is strongly associated with senescence ${ }^{8,11,54}$; Egr1, depending on the cellular context, stimulates or reduces proliferation. The data presented here suggest a molecular connection between Egr1 and VPA in the context of early epigenomic remodeling and between VPA and Bmi1 in the regulation of hyperproliferation during chondrogenesis. The dose dependent effects of VPA on chondrogenesis we report are consistent with epigenetic (de)regulation, as epigenetic modulation most often produces hypomorphic (e.g. variegation or gene-dosage effects) rather than all-or-none effects. In light of findings reported by us and others, it is likely that chondrogenesis (and consequently 
skeletogenesis) are specifically sensitive to VPA, because of characteristic biological features associated with endochondral ossification, among which rapid proliferation and hypertrophy.

In conclusion, VPA causes an enhanced susceptibility to replication stress and induces a Trp53dependent replicative block and senescence-like response in hyperproliferating chondrocytes. Our findings have potentially important repercussion for the clinical use of VPA: the success of combination therapy of e.g. both solid and hematological cancers with VPA and topoisomerase inhibitors ${ }^{48,55}$ may directly relate to enhanced sensitization of rapidly dividing cancer cells for replication stress, in analogy to the effects on chondrogenic progenitors we report here. These combined observations should provide important leads for detailed analysis of the molecular basis of the role of VPA on replication and provide relevant possibilities for therapeutic applications in cancer.

\section{Acknowledgements}

The authors received financial support from the European Molecular Biology Organization (Germany) ASTF5-2009; the Dutch Science Organization (ZonMW-NWO) VIDI grant 016.046.362, the National Rheuma Foundation (Reumafonds) grant LLP14 and transnational University of Limburg (tUL) grant to JWV.

\section{References}

1. Basu A, Wezeman FH: Developmental toxicity of valproic acid during embryonic chick vertebral chondrogenesis. Spine (Phila Pa 1976) 2000, 25:2158-2164.

2. Dansky LV, Finnell RH: Parental epilepsy, anticonvulsant drugs, and reproductive outcome: epidemiologic and experimental findings spanning three decades; 2: Human studies. Reprod Toxicol 1991, 5:301-335.

3. Faiella A, Wernig M, Consalez GG, Hostick U, Hofmann C, Hustert E, Boncinelli E, Balling R, Nadeau JH: A mouse model for valproate teratogenicity: parental effects, homeotic transformations, and altered HOX expression. Hum Mol Genet 2000, 9:227-236.

4. Kawanishi CY, Hartig P, Bobseine KL, Schmid J, Cardon M, Massenburg G, Chernoff N: Axial skeletal and Hox expression domain alterations induced by retinoic acid, valproic acid, and bromoxynil during murine development. J Biochem Mol Toxicol 2003, 17:346-356.

5. $\quad$ Perucca E: Birth defects after prenatal exposure to antiepileptic drugs. Lancet Neurol 2005, 4:781786.

6. Samren EB, van Duijn CM, Christiaens GC, Hofman A, Lindhout D: Antiepileptic drug regimens and major congenital abnormalities in the offspring. Ann Neurol 1999, 46:739-746.

7. Jung GA, Yoon JY, Moon BS, Yang DH, Kim HY, Lee SH, Bryja V, Arenas E, Choi KY: Valproic acid induces differentiation and inhibition of proliferation in neural progenitor cells via the beta-cateninRas-ERK-p21Cip/WAF1 pathway. BMC Cell Biol 2008, 9:66.

8. Yu IT, Park JY, Kim SH, Lee JS, Kim YS, Son H: Valproic acid promotes neuronal differentiation by induction of proneural factors in association with H4 acetylation. Neuropharmacology 2009, 56:473480.

9. Chen $Y$, Pan RL, Zhang XL, Shao JZ, Xiang LX, Dong XJ, Zhang GR: Induction of hepatic differentiation of mouse bone marrow stromal stem cells by the histone deacetylase inhibitor VPA. $J$ Cell Mol Med 2008.

10. Cho HH, Park HT, Kim YJ, Bae YC, Suh KT, Jung JS: Induction of osteogenic differentiation of human mesenchymal stem cells by histone deacetylase inhibitors. J Cell Biochem 2005, 96:533542.

11. Li XN, Shu Q, Su JM, Perlaky L, Blaney SM, Lau CC: Valproic acid induces growth arrest, apoptosis, and senescence in medulloblastomas by increasing histone hyperacetylation and regulating expression of p21Cip1, CDK4, and CMYC. Mol Cancer Ther 2005, 4:1912-1922. 
12. Schwartz C, Palissot V, Aouali N, Wack S, Brons NH, Leners B, Bosseler M, Berchem G: Valproic acid induces non-apoptotic cell death mechanisms in multiple myeloma cell lines. Int $J$ Oncol 2007 , 30:573-582.

13. Shabbeer S, Kortenhorst MS, Kachhap S, Galloway N, Rodriguez R, Carducci MA: Multiple Molecular pathways explain the anti-proliferative effect of valproic acid on prostate cancer cells in vitro and in vivo. Prostate 2007, 67:1099-1110.

14. Shen WT, Wong TS, Chung WY, Wong MG, Kebebew E, Duh QY, Clark OH: Valproic acid inhibits growth, induces apoptosis, and modulates apoptosis-regulatory and differentiation gene expression in human thyroid cancer cells. Surgery 2005, 138:979-984; discussion 984-975.

15. Murabe M, Yamauchi J, Fujiwara Y, Hiroyama M, Sanbe A, Tanoue A: A novel embryotoxic estimation method of VPA using ES cells differentiation system. Biochem Biophys Res Commun 2007, 352:164-169.

16. De Sarno P, Li X, Jope RS: Regulation of Akt and glycogen synthase kinase-3 beta phosphorylation by sodium valproate and lithium. Neuropharmacology 2002, 43:1158-1164.

17. Rinaldi T, Kulangara K, Antoniello K, Markram H: Elevated NMDA receptor levels and enhanced postsynaptic long-term potentiation induced by prenatal exposure to valproic acid. Proc Natl Acad Sci U S A 2007, 104:13501-13506.

18. Gottlicher M, Minucci S, Zhu P, Kramer OH, Schimpf A, Giavara S, Sleeman JP, Lo Coco F, Nervi C, Pelicci PG, Heinzel T: Valproic acid defines a novel class of HDAC inhibitors inducing differentiation of transformed cells. Embo J 2001, 20:6969-6978.

19. Bernstein BE, Mikkelsen TS, Xie X, Kamal M, Huebert DJ, Cuff J, Fry B, Meissner A, Wernig M, Plath $\mathrm{K}$, et al: A bivalent chromatin structure marks key developmental genes in embryonic stem cells. Cell 2006, 125:315-326.

20. Akasaka T, Kanno M, Balling R, Mieza MA, Taniguchi M, Koseki H: A role for mel-18, a Polycomb group-related vertebrate gene, during theanteroposterior specification of the axial skeleton. Development 1996, 122:1513-1522.

21. del Mar Lorente M, Marcos-Gutierrez C, Perez C, Schoorlemmer J, Ramirez A, Magin T, Vidal M: Loss- and gain-of-function mutations show a polycomb group function for Ring1A in mice. Development 2000, 127:5093-5100.

22. Hanson RD, Hess JL, Yu BD, Ernst P, van Lohuizen M, Berns A, van der Lugt NM, Shashikant CS, Ruddle FH, Seto M, Korsmeyer SJ: Mammalian Trithorax and polycomb-group homologues are antagonistic regulators of homeotic development. Proc Natl Acad Sci U S A 1999, 96:14372-14377.

23. Okada A, Aoki Y, Kushima K, Kurihara H, Bialer M, Fujiwara M: Polycomb homologs are involved in teratogenicity of valproic acid in mice. Birth Defects Res A Clin Mol Teratol 2004, 70:870-879.

24. He Z, Li J, Zhen C, Feng L, Ding X: Knockdown of p53 by RNAi in ES cells facilitates RA-induced differentiation into muscle cells. Biochem Biophys Res Commun 2005, 335:676-683.

25. Goodarzi AA, Noon AT, Deckbar D, Ziv Y, Shiloh Y, Löbrich M, Jeggo PA: ATM Signaling Facilitates Repair of DNA Double-Strand Breaks Associated with Heterochromatin. Molecular Cell 2008, 31:167-177.

26. Dimri GP, Lee X, Basile G, Acosta M, Scott G, Roskelley C, Medrano EE, Linskens M, Rubelj I, Pereira-Smith $\mathrm{O}$, et al.: A biomarker that identifies senescent human cells in culture and in aging skin in vivo. Proc Natl Acad Sci U S A 1995, 92:9363-9367.

27. Atsumi T, Miwa Y, Kimata K, Ikawa Y: A chondrogenic cell line derived from a differentiating culture of AT805 teratocarcinoma cells. Cell Differ Dev 1990, 30:109-116.

28. Campisi J, d'Adda di Fagagna F: Cellular senescence: when bad things happen to good cells. Nat Rev Mol Cell Biol 2007, 8:729-740.

29. Di Leonardo A, Linke SP, Clarkin K, Wahl GM: DNA damage triggers a prolonged p53-dependent G1 arrest and long-term induction of Cip1 in normal human fibroblasts. Genes Dev 1994, 8:25402551.

30. Wouters BG, Koritzinsky M: Hypoxia signalling through mTOR and the unfolded protein response in cancer. Nat Rev Cancer 2008, 8:851-864.

31. Mallette FA, Gaumont-Leclerc M-F, Ferbeyre G: The DNA damage signaling pathway is a critical mediator of oncogene-induced senescence. Genes Dev 2007, 21:43-48.

32. Di Micco R, Fumagalli M, Cicalese A, Piccinin S, Gasparini P, Luise C, Schurra C, Garre M, Nuciforo PG, Bensimon A, et al: Oncogene-induced senescence is a DNA damage response triggered by DNA hyper-replication. Nature 2006, 444:638-642.

33. Bartkova J, Rezaei N, Liontos M, Karakaidos P, Kletsas D, Issaeva N, Vassiliou LV, Kolettas E, Niforou K, Zoumpourlis VC, et al: Oncogene-induced senescence is part of the tumorigenesis barrier imposed by DNA damage checkpoints. Nature 2006, 444:633-637.

34. Kuilman T, Peeper DS: Senescence-messaging secretome: SMS-ing cellular stress. Nat Rev Cancer 2009, 9:81-94.

35. Fumagalli M, d'Adda di Fagagna F: SASPense and DDRama in cancer and ageing. Nat Cell Biol 2009, 11:921-923. 
36. Honda R, Yasuda $\mathrm{H}$ : Association of p19(ARF) with Mdm2 inhibits ubiquitin ligase activity of Mdm2 for tumor suppressor p53. Embo J 1999, 18:22-27.

37. Kamijo T, Weber JD, Zambetti G, Zindy F, Roussel MF, Sherr CJ: Functional and physical interactions of the ARF tumor suppressor with p53 and Mdm2. Proc Natl Acad Sci U S A 1998, 95:8292-8297.

38. Berns K, Hijmans EM, Mullenders J, Brummelkamp TR, Velds A, Heimerikx M, Kerkhoven RM, Madiredjo M, Nijkamp W, Weigelt B, et al: A large-scale RNAi screen in human cells identifies new components of the p53 pathway. Nature 2004, 428:431-437.

39. Kortenhorst MS, Isharwal S, van Diest PJ, Chowdhury WH, Marlow C, Carducci MA, Rodriguez R, Veltri RW: Valproic acid causes dose- and time-dependent changes in nuclear structure in prostate cancer cells in vitro and in vivo. Mol Cancer Ther 2009, 8:802-808.

40. Zuscik MJ, Hilton MJ, Zhang $X$, Chen D, O'Keefe RJ: Regulation of chondrogenesis and chondrocyte differentiation by stress. $J$ Clin Invest 2008, 118:429-438.

41. Okada A, Shiomi T, Aoki Y, Fujiwara M: Phenytoin stimulates chondrogenic differentiation in mouse clonal chondrogenic EC cells, ATDC5. J Toxicol Sci 2005, 30:145-156.

42. Fischer S, Prykhozhij S, Rau MJ, Neumann CJ: Mutation of zebrafish caf-1b results in $S$ phase arrest, defective differentiation, and p53-mediated apoptosis during organogenesis. Cell Cycle 2007, 6:2962-2969.

43. Courtois-Cox S, Jones SL, Cichowski K: Many roads lead to oncogene-induced senescence. Oncogene 2008, 27:2801-2809.

44. Denoyelle C, Abou-Rjaily G, Bezrookove V, Verhaegen M, Johnson TM, Fullen DR, Pointer JN, Gruber SB, Su LD, Nikiforov MA, et al: Anti-oncogenic role of the endoplasmic reticulum differentially activated by mutations in the MAPK pathway. Nat Cell Biol 2006, 8:1053-1063.

45. Lavelle $D$, Chen $Y H$, Hankewych $M$, DeSimone J: Histone deacetylase inhibitors increase p21(WAF1) and induce apoptosis of human myeloma cell lines independent of decreased IL-6 receptor expression. Am J Hematol 2001, 68:170-178.

46. Bown CD, Wang JF, Chen B, Young LT: Regulation of ER stress proteins by valproate: therapeutic implications. Bipolar Disord 2002, 4:145-151.

47. $\mathrm{Na} L$, Wartenberg $\mathrm{M}$, Nau $\mathrm{H}$, Hescheler J, Sauer $\mathrm{H}$ : Anticonvulsant valproic acid inhibits cardiomyocyte differentiation of embryonic stem cells by increasing intracellular levels of reactive oxygen species. Birth Defects Res A Clin Mol Teratol 2003, 67:174-180.

48. Das CM, Aguilera D, Vasquez H, Prasad P, Zhang M, Wolff JE, Gopalakrishnan V: Valproic acid induces p21 and topoisomerase-II (alpha/beta) expression and synergistically enhances etoposide cytotoxicity in human glioblastoma cell lines. J Neurooncol 2007, 85:159-170.

49. Narita M, Krizhanovsky V, Nunez S, Chicas A, Hearn SA, Myers MP, Lowe SW: A novel role for high-mobility group a proteins in cellular senescence and heterochromatin formation. Cell 2006, 126:503-514.

50. Yamada T, Fischle W, Sugiyama T, Allis CD, Grewal SI: The nucleation and maintenance of heterochromatin by a histone deacetylase in fission yeast. Mol Cell 2005, 20:173-185.

51. Massa V, Cabrera RM, Menegola E, Giavini E, Finnell RH: Valproic acid-induced skeletal malformations: associated gene expression cascades. Pharmacogenet Genomics 2005, 15:787-800.

52. Jacobs JJ, Kieboom K, Marino S, DePinho RA, van Lohuizen M: The oncogene and Polycombgroup gene bmi-1 regulates cell proliferation and senescence through the ink4a locus. Nature 1999, 397:164-168.

53. Itahana K, Zou Y, Itahana Y, Martinez JL, Beausejour C, Jacobs JJ, Van Lohuizen M, Band V, Campisi J, Dimri GP: Control of the replicative life span of human fibroblasts by p16 and the polycomb protein Bmi-1. Mol Cell Biol 2003, 23:389-401.

54. Adamson ED, Yu J, Mustelin T: Co-factors p300 and CBP catch Egr1 in their network. Prostate 2005, 63:407-410.

55. Munster P, Marchion D, Bicaku E, Schmitt M, Lee JH, DeConti R, Simon G, Fishman M, Minton S, Garrett $C$, et al: Phase I trial of histone deacetylase inhibition by valproic acid followed by the topoisomerase II inhibitor epirubicin in advanced solid tumors: a clinical and translational study. $J$ Clin Oncol 2007, 25:1979-1985. 
Chapter 6

\section{Supplemental Table 1 Primers used for qRT rtPCR}

\begin{tabular}{|c|c|c|}
\hline \multirow[t]{2}{*}{ Cyclophillin } & Forward & caaatgctggaccaaacacaa \\
\hline & Reverse & ttcaccttcccaaagaccacat \\
\hline \multirow[t]{2}{*}{ Collagen 2a1 } & Forward & caagaaccctgctcgcactt \\
\hline & Reverse & ccgetcttccactcggg \\
\hline \multirow[t]{2}{*}{ Aggrecan } & Forward & catgagagaggcgaatggaa \\
\hline & Reverse & tgatctcgtagcgatctttcttct \\
\hline \multirow[t]{2}{*}{ Collagen $10 \mathrm{a} 1$} & Forward & catgcctgatggcttcataaa \\
\hline & Reverse & aagcagacacgggcatacct \\
\hline \multirow[t]{2}{*}{ Glb1 } & Forward & gagaaagttcaagacagtggctg \\
\hline & Reverse & ccattgggacacaggataccc \\
\hline \multirow[t]{2}{*}{ II-6 } & Forward & gctaccaaactggatataatcaggaaa \\
\hline & Reverse & cttgttatcttttaagttgttcttcatgtactc \\
\hline \multirow[t]{2}{*}{ Chop } & Forward & gggccaacagaggtcacac \\
\hline & Reverse & cttcatgcgttgcttccca \\
\hline \multirow[t]{2}{*}{ P19ARF } & Forward & aagacggccttgcaggtcat \\
\hline & Reverse & ctagtaccggaggcatcttgga \\
\hline \multirow[t]{2}{*}{ Sox9 } & Forward & agtacccgcacctgcacaac \\
\hline & Reverse & tacttgtagtccgggtggtctttc \\
\hline \multirow[t]{2}{*}{ Runx2 } & Forward & gacgaggcaagagtttcacc \\
\hline & Reverse & ggaccgtccactgtcacttt \\
\hline \multirow[t]{2}{*}{ Ezh2 } & Forward & gaaaaaagatgagacgtccagctc \\
\hline & Reverse & gaggttcaatatttggcttcatctttat \\
\hline \multirow[t]{2}{*}{ Bmi1 } & Forward & tggagaagaatggeccact \\
\hline & Reverse & gcaagttggccgaactctgt \\
\hline \multirow[t]{2}{*}{ Rnf2 } & Forward & tcaggccccatccaactct \\
\hline & Reverse & ggcattgcctgaagtctttatgt \\
\hline \multirow[t]{2}{*}{ Ring1 } & Forward & gccacagtggatcatctctcc \\
\hline & Reverse & tgctgctgcctcctctcc \\
\hline \multirow[t]{2}{*}{ Egr1 } & Forward & agccgagcgaacaaccctat \\
\hline & Reverse & tgtcagaaaaggactctgtggtca \\
\hline
\end{tabular}


Chapter 7

General Discussion 
PcG proteins are epigenetic regulators, first identified in Drosophila melanogaster as regulators of segmental identity. PcG mutant Drosophila and mice show homeotic transformations along the antero-posterior axis, in mice resulting in vertebral transformations ${ }^{1-4}$. The skeletal phenotype of PcG mutant mice has been ascribed to deregulation of HOX genes, classical PcG target genes, but the precise mechanism behind the development of segment identity shifts is currently unknown. Chondrogenesis is a multistep process that forms the cartilage matrix that will eventually ossify to form skeletal elements ${ }^{1}$. We speculated that PcG proteins play a critical role in mediating the epigenomic responses to a changing microenvironment (here inducing chondrogenic differentiation), and thus in cell fate decisions. In this thesis various techniques were used to study chondrogenic differentiation and in particular the role of PcG proteins herein.

We find that a hypoxic response during chondrogenesis is an important part of cellular responses to changing environmental conditions during chondrogenic differentiation (chapter 3 ). In addition, we find that interference with epigenomic remodelling during chondrogenesis severely affects chondrogenic capacity. This aspect was studied by exposure to Valproic acid (VPA), a reported HDAC inhibitor and by functional loss of important epigenetic regulators of the Polycomb Group (PcG) of proteins. Finally we find that induction of the immediate early response gene EGR1 is required for epigenomic remodelling during the early phases of differentiation.

\section{Gene-environment interactions in chondrogenic differentiation}

In a novel in vivo model we show that placing activated mesenchymal stem cells, derived from a periosteal callus (PC), into an induced lesion, repairs the osteochondral defect (Chapter 3 ). Without supplemental addition of growth factors, relevant pathways are activated and this model recapitulates the different stages of enchondral ossification. Lack of vascularization makes cartilage a very hypoxic tissue environment ${ }^{2}$. In line with this notion we observed increased mRNA expression of HIF-1 $\alpha$ and its transcriptional targets VEGF and GAPDH (Chapter 3). Under normoxic conditions HIF-1 $\alpha$ is hydroxylated by prolyl hydroxylases and consequently ubiquitytlated by the von Hippel Lindau gene product VHL, and targeted for proteasomal degradation ${ }^{3}$. Under

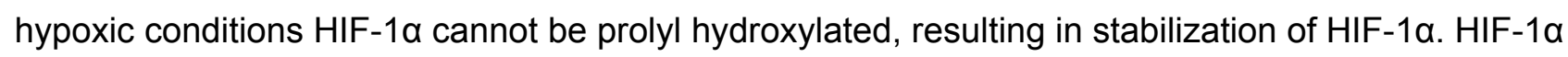
helps cells adapt to a hypoxic environment, by activating downstream targets, involved in e.g. cellular survival, anaerobic metabolism and vascularization. We observed stabilization of the HIF$1 \alpha$ at the protein level in non-hypertrophic chondrocytes. The absence of HIF-1a protein in hypertrophic chondrocytes is in good agreement with the onset of vascularization, and consequent oxygenation in the hypertrophic region of the growth plate.

In this study, we observed a down regulation of BMP-2, -4 , and -7 , while increased levels were found at the protein level, throughout callus formation and osteochondral differentiation (Chapter 3). Studies on BMP proteins in callus formation and osteochondral differentiation are not in agreement; while Yaoita et al. reports that levels BMPs remain unaffected in response to inducing a fracture, Kloen et al. reports a positive staining for BMPs in human callus. Others show an up- 
regulation of BMP-2 24 hours after surgical release of the periosteum ${ }^{4}$. The results in the study presented here, show regulation of BMPs at both the transcriptional as well as post-transcriptional level. Recently, select mRNAs were found to be preferentially translated under hypoxic conditions through increased association of mRNAs with polysomes ${ }^{5}$. Whether BMPs are subject to preferential translation under hypoxia remains to be determined. Combined, these results strongly suggest that the microenvironment, in which the PC is placed, results in activation of pathways relevant to chondrogenic differentiation and to adaptation to the hypoxic surroundings.

\section{Dual role for Egr1 during chondrogenic differentiation}

Murine ATDC5 mesenchymal stem cells activated to undergo chondrogenesis reiterate the welldescribed multi-stage differentiation program, and are widely used to study chondrogenic differentiation. We report for the first time that in response to a changing the microenvironment (i.e. Growth factors in differentiation medium) rapidly and transiently induces EGR1 and other immediate early genes (IEGs). Knockdown of EGR1 in ATDC5 cells severely impedes two different characteristics of ATDC5 chondrogenesis.

Firstly, the ability of Egr1-LOF (loss-of-function) cells to undergo chondrogenic differentiation is severely affected. In line with this, promoters of RUNX2 and SOX9, transcription factors critical for different aspects of chondrogenesis were identified as direct targets of Egr1 (Chapter 4); in the absence of Egr1, activation of these targets is blunted upon induction of differentiation. Egr1 was shown to recruit HAT activity ${ }^{6}$, which is in good agreement with early abnormal global histone acetylation, observed in Egr1-LOF cells. Currently, we do not know whether Egr1 directly activates transcription of these targets, or whether recruitment of HAT activity to relevant promoters creates an epigenomic state permissive of transcriptional activation.

Secondly, ATDC5 cells induced to differentiate undergo a transient phase of rapid progenitor proliferation; Egr1-LOF cells fail to undergo this differentiation induced expansion phase and undergo premature senescence, specifically during the hyperproliferative phase of chondrogenic differentiation (Chapter 5). Senescence is a protective mechanism to prevent oncogenic transformation of cells that accumulate excessive damage. Indeed, loss of Egr1 function results in accumulation of DNA damage and expression of numerous senescence-associated marker genes (Chapter 4). Intriguingly, this proliferative block resembles features of oncogene-induced senescence ${ }^{9-11}$. Expression of oncogenes results in hyperproliferation, and thus hyperreplication, to such an extent that the cells cannot maintain proper regulation of replication ${ }^{7-9}$. Consequently, replication forks stall, and DNA damage accumulates. Next, activated DNA damage responses (DDR) induce a cell cycle arrest in S-phase and irreversible senescence, which are dependent on the DDR ${ }^{7}$.

Our findings position Egr1 at the onset of a proliferative burst. Hence Egr1 fulfils a regulatory role in lineage-specific transcriptional re-programming, as well as in transit progenitor expansion. 


\section{Loss of Egr1-function affects epigenomic remodeling}

Apart from lack of global acetylation, examination of other histone modifications revealed a significantly increased trimethylation of H3K4, H3K9 and H3K27, both in control and Egr1-LOF cultures. In control cells trimethyl levels increase during the phase of transit amplification. In addition, an exaggerated increase in expression of these modifications is observed in Egr1-LOF cultures. These epigenetic trimethylation marks are seemingly in conflict with each other, as for instance $\mathrm{H} 3 \mathrm{~K} 9$ and $\mathrm{H} 3 \mathrm{~K} 27 \mathrm{me} 3$ are associated with transcriptionally repressed/silent DNA, whereas $\mathrm{H} 3 \mathrm{~K} 4 \mathrm{me} 3$ is often found at loci which are actively expressed. The observed increase in global trimethylation correlates with a period of enhanced cell stress and suggest that besides in transcriptional regulation, these modifications are implicated in other, as of yet unkown regulatory processes. It has been reported that the heterochromatin markers HP1 and H3K9me3 both accumulate in distinct nuclear foci during or in response to cellular senescence ${ }^{10}$. These senescence-associated heterochromatin foci (SAHF) appear during replication stress induced senescence. We find that expression of KAP1 precedes the accumulation of H3K9me3 in Egr1LOF cells (Chapter 4). Kap1, a factor known to directly bind HP1, was reported to be required to help resolve DNA damage specifically in heterochromatin ${ }^{11}$. Together, H3K9me3 and Kap1 may be involved in protection against replication induced DNA damage. Also increased levels of H3K4me3 and H3K27me3 have been associated with cellular stress responses, i.e. hypoxia or induction of replication stress (Johnson et al. +FS, PP, JWV; unpublished data). H3K4me3 was reported to be essential for Rag2-mediated $V(D) J$ recombination in $B$ and $T$ cells, and is linked to DDR-induced cellular responses via ING family proteins. In line with this notion, we also find that VPA, a reported HDAC inhibitor, severely affects chondrogenic differentiation. Like loss of Egr1 and PcG function (chapter $5+$ see below) VPA treatment leaves the ATDC5 cells unable to hyperproliferate and activates senescence response. The observed cellular stress responses again correlate with globally enhanced histone trimethylation. Interestingly, VPA treatment affects proliferation, but not on differentiation, in a TP53-dependent manner (Chapter 6). These findings are in agreement with similar observations in zebrafish ${ }^{12}$. Combined these observations suggest that several important processes, including a proliferative response and transcriptomic reprogramming, are part of a coordinated response during chondrogenesis. Our findings support the importance of epigenomic remodelling for chondrogenesis. In addition, they suggest that global trimethylation may be part of a more generalized stress response.

\section{Egr1 initiates early epigenomic reprogramming via activation of PcG proteins}

In silico analysis of Egr1 binding sites predicted PcG proteins as potential target genes. Interestingly, we observed that Egr1-LOF affects expression of Ezh2 and Bmi1, and in line with these findings we were able to immunoprecipitate PcG promoter sequences with specific anti-Egr1 antibodies (Chapter 4). Both Ezh2 and Bmi1 have been associated with cell-cycle regulation, as Ezh2 is a transcriptional target of $E 2 F^{13}$, and Bmi1 transcriptionally regulates the CDKN2/INK4A 
Chapter 7

locus, which encodes the cell-cycle regulators $\mathrm{P} 16^{\mathrm{ARF}}$ and $\mathrm{P} 14^{\mathrm{INK} 4 \mathrm{a}}{ }^{14}$. This suggests that Egr1 is involved in transcriptional activation of PcG genes and that Egr1 thereby recruits epigenetic modulators essential for chondrogenic differentiation. Furthermore, a comparison between published PcG targets and predicted Egr1 consensus binding sites revealed potential common targets. Relevantly, two hours post induction of differentiation (pid) we observe a strong correlation between H3K27me3 marks at loci and the absence of Egr1 binding, and inversely, between the presence of $\mathrm{H} 3 \mathrm{~K} 4 \mathrm{me} 3$ marks and local enrichment of Egr1, suggesting that PRCs regulate accessibility of Egr1 to promoters. Combined, this data reveal a number of potential mechanistic interactions between Egr1 and PRCs.

\section{The PRC1 complex regulates transit amplification and transcriptional reprogramming of chondrogenic progenitors}

Analogously to Egr1-LOF cells, loss of PRC1 function results in failure to properly differentiate. In addition we observe a significant intra-S-phase arrest and induction of senescence. In agreement with the intra-S-phase arrest, BrdU incorporation revealed an inability to properly replicate DNA in Bmi1-kd cells. Under differentiation conditions, PRC1-LOF cells accumulate massive amounts of DNA damage, as measured by phosphorylated H2A.X, Chk2 and Kap1 (Chapter 5). Again, as for loss of Egr1 function, these results show strong similarity to OIS, suggesting that hyperproliferation induced replication stress is the underlying cause of the phenotype observed in PRC-LOF.

All chondrogenic cell lines available showed strong staining for senescence-associated bGalactosidase (SAbGAL). Furthermore, intercalated discs and in growth plates of elongating bones of WT mice show presence of SAbGAL. In good agreement with in vitro findings, RING1AKO mice show an increased hypertrophic zone (HZ) (Chapter 5). Importantly, Runx, an oncogene, reported to induce senescence, is implicated in hypertrophic differentiation. Together, our findings suggest that endochondral ossification may naturally terminate in a senescence-like phenotype. To the best of our knowledge, this is the first report describing an important role for hyperproliferation checkpoints in a naturally occurring differentiation process during development. Thusfar these cellular stress responses (DDR, intra-S arrest and senescence) have mainly been associated with oncogene-induced hyperproliferation and consequential checkpoint activation and cell cycle exit. We performed extensive microarray analysis on Bmi1-kd and control cells, and we found that transcriptional re-profiling in control cells intensifies during transit amplification. Transcriptomicl differences between Bmi1-kd and control cells increase dramatically at this time. Unexpectedly, we observed that equal amounts of genes are up- or down-regulated at 6 days pid in Bmi1-kd cells compared to control cells (Chapter 5). Expression profiling of genes most extremely deregulated revealed a number of different response clusters, suggesting global deregulation of transcriptional responses rather than deregulation of specific relevant processes. Combined these finding illustrate that at the time of proliferative amplification, the transcriptome is heavily reorganized to 
facilitate chondrogenic differentiation, and that PRC1 is critical for regulating these transcriptional changes.

Cells undergoing differentiation require coordinated regulation of DNA-templated processes, among which transcription and replication. Using Immunofluorescence, we studied transcription, replication and the presence of DNA damage in respect to each other. Intriguingly, transcription and replication activity appear to be separated in space and time inside the nucleus, suggesting that the genome is partitioned into regions that are either actively transcribing or actively replicating DNA under normal conditions (Chapter 5). This unexpected finding is in good agreement with an earlier report on dynamic segregation of mRNA and DNA production throughout S-phase ${ }^{15}$. Remarkably, this spatial segregation of transcription and replication is lost in PRC1-kd cultures, suggesting that concerted regulation of the two DNA-templated processes is regulated by PRC1. Moreover, in PRC1-LOF cells, DNA damage occurs at sites of de novo DNA synthesis. The majority of DNA damage in replicating cells, even under normal conditions, probably comes from highly frequent collisions between replication forks and transcription machineries, as both processes move along the same template ${ }^{16-18}$. Our findings suggest that damage in PRC1-LOF cells accumulates as a result of failure to tune replication to transcription and vice versa.

Bmi1-deficient cells are positive for geminin expression and control cells are not (Chapter 5). This inverse correlation between Gmnn and Bmi1/Rnf2 chromatin association during mid-S is in perfect agreement with the recently identified ubiquitin-mediated induction of Gmnn degradation by Bmi1/Rnf2 ${ }^{19}$. Chromatin dissociation of Bmi1/Rnf2 is likely controlled by post-translational modification (PTM). In line with this idea, we recently observed a positive correlation between PRC-phosphorylation, PRC1/chromatin dissociation and Gmnn stabilization under various conditions of cell stress (JWV, YT unpublished observations). Stress signaling induces chromatin dissociation of PRC1 members (Bmi1, Ring and CBX proteins; ${ }^{22}$ ). In contrast, Phc1/Rae28 ${ }^{22}$, through which Gmnn is associated with chromatin, undergoes a nuclear redistribution under these conditions. Taken together, these observations point to $\mathrm{Gmnn}$ as a likely candidate for coordinated control of transcription and replication in the context of PRC1 function.

Based on these findings, we propose a model in which the PRC/geminin interactions maintain a dynamic switch to spatially and temporally segregate transcription and replication: H3K27me3mediated recruitment of the core complex CBX/Rnf2/Bmi1 installs repressive H2AK119ub1 marks, which interfere with transcription initiation, possibly through recruitment of additional PRC (associated) proteins ${ }^{20}$. Local presence of Rnf2/Bmi1 E3 ligase activity would target Gmnn for degradation, relieving a potential replication block. Conversely, loss of Bmi1/Rnf2 stabilizes GMNN, hence blocking replication, yet permitting transcription (see figure 1, chapter 2). 
Chapter 7

\section{Conclusions and future perspectives}

The research in this thesis provides novel insights to mechanisms mediating gene-environment interactions. Changing the environment to induce chondrogenic differentiation rapidly induces a wide array of responses (Chapters 3, 4 and 5). Egr1, while expressed only shortly, activates chondrogenic programs and initiates epigenomic remodeling. Initiating epigenomic remodeling by Egr1 is critical for subsequent transit amplification of chondrogenic progenitors, at least in part via regulation of PRCs (Chapter 4). The importance of epigenomic remodeling during transit amplification is stressed by the findings that the histone deacetylase inhibitor valproic acid results in replicative senescence and a failure to differentiate (Chapter 6). Detailed analysis of PRC function during chondrogenesis revealed novel insights in coordinated regulation of transcription and replication and proposes a role for PRCs therein (Chapter 5). Loss of PRC function results in replication stress, in accumulation of DNA damage and induction of replicative senescence (Chapter 5). Although our studies have increased our fundamental understanding of geneenvironment interactions and uncovered a novel role for PcG proteins in coordinating transcription and replication, many questions remain. May Egr1 binding to DNA define genomic regions with active transcription or replication? Is Egr1 binding restricted to promoter regions? How does Egr1 binding to DNA relate to specific histone modifications, and to PRC occupation? Which genes are regulated by Egr1 or PRCs? Does PRC function in replication timing, and is Egr1 involved? Is enrichment of specific histone modifications and PRC occupation dynamic during S-phase, when transcription and replication are co-ordinately regulated? Are potential targets dynamically regulated in replication timing the genes that accumulate DNA damage in PRC- or Egr1-LOF? How, exactly does geminin contribute to Polycomb biology? Genome wide chromatin immunoprecipitation (ChIP-seq) of Egr1, specific histone modifications and PcG proteins during different stages of differentiation, will be important to map common targets. In combination with microarrays of Egr1-LOF versus control cells and the available Bmi1-LOF microarray data, these data sets will shed light on the importance of regulating these targets, in the context of transcription. Replication timing involves coordinated regulation of transcription and replication (discussed in Chapter 2). This process provides a relevant starting point to study the coordinate regulation of transcription and replication by PRCs. Genome wide ChIPs for active RNA polymerase II, BrdU, geminin and specific histone modifications in different stages of S-phase, as a function of PRC expression will help studying the proposed model (Chapters 2 and 7). Our findings suggest a novel aspect of Polycomb biology during skeletogenesis, and to the importance of proliferation and tissue expansion in positioning along the A-P axis. Further, premature termination of differentiation may account for the phenotype observed in PcG null-mutants.

Overall, our observations provide important connecting points for further research, and contribute to mechanistic understanding concerning the role of PcG proteins in embryogenesis and oncogenesis. Finding answers to the above questions will be of significant importance to enhance our as of yet incomplete understanding of epigenomic regulation in development and disease. 


\section{References}

1. Mackie, E. J., Ahmed, Y. A., Tatarczuch, L., Chen, K. S. \& Mirams, M. Endochondral ossification: how cartilage is converted into bone in the developing skeleton. Int J Biochem Cell Biol 40, 46-62 (2008).

2. Schipani, E. Hypoxia and HIF-1alpha in chondrogenesis. Ann N Y Acad Sci 1068, 66-73 (2006).

3. Lee, J. W., Bae, S. H., Jeong, J. W., Kim, S. H. \& Kim, K. W. Hypoxia-inducible factor (HIF-1)alpha: its protein stability and biological functions. Exp Mol Med 36, 1-12 (2004).

4. Simon, T. M., Van Sickle, D. C., Kunishima, D. H. \& Jackson, D. W. Cambium cell stimulation from surgical release of the periosteum. J Orthop Res 21, 470-80 (2003).

5. Koritzinsky, M. et al. Gene expression during acute and prolonged hypoxia is regulated by distinct mechanisms of translational control. Embo J 25, 1114-25 (2006).

6. Silverman, E. S. et al. cAMP-response-element-binding-protein-binding protein (CBP) and p300 are transcriptional co-activators of early growth response factor-1 (Egr-1). Biochem J 336 ( Pt 1), 183-9 (1998).

7. Bartkova, J. et al. Oncogene-induced senescence is part of the tumorigenesis barrier imposed by DNA damage checkpoints. Nature 444, 633-7 (2006).

8. Di Micco, R. et al. Oncogene-induced senescence is a DNA damage response triggered by DNA hyper-replication. Nature 444, 638-42 (2006).

9. Kilbey, A., Terry, A., Cameron, E. R. \& Neil, J. C. Oncogene-induced senescence: an essential role for Runx. Cell Cycle 7, 2333-40 (2008).

10. Funayama, R. \& Ishikawa, F. Cellular senescence and chromatin structure. Chromosoma 116, 43140 (2007).

11. Goodarzi, A. A. et al. ATM signaling facilitates repair of DNA double-strand breaks associated with heterochromatin. Mol Cell 31, 167-77 (2008).

12. Fischer, S., Prykhozhij, S., Rau, M. J. \& Neumann, C. J. Mutation of zebrafish caf-1b results in $S$ phase arrest, defective differentiation, and p53-mediated apoptosis during organogenesis. Cell Cycle 6, 2962-9 (2007).

13. Attwooll, C. et al. A novel repressive E2F6 complex containing the polycomb group protein, EPC1, that interacts with EZH2 in a proliferation-specific manner. J Biol Chem 280, 1199-208 (2005).

14. Gil, J. \& Peters, G. Regulation of the INK4b-ARF-INK4a tumour suppressor locus: all for one or one for all. Nat Rev Mol Cell Biol 7, 667-77 (2006).

15. Wei, X. et al. Segregation of transcription and replication sites into higher order domains. Science 281, 1502-6 (1998).

16. Brewer, B. J. When polymerases collide: replication and the transcriptional organization of the E. coli chromosome. Cell 53, 679-86 (1988).

17. Mirkin, E. V. \& Mirkin, S. M. Replication fork stalling at natural impediments. Microbiol Mol Biol Rev 71, 13-35 (2007).

18. Rudolph, C. J., Dhillon, P., Moore, T. \& Lloyd, R. G. Avoiding and resolving conflicts between DNA replication and transcription. DNA Repair (Amst) 6, 981-93 (2007).

19. Ohtsubo, M. et al. Polycomb-group complex 1 acts as an E3 ubiquitin ligase for Geminin to sustain hematopoietic stem cell activity. Proc Natl Acad Sci U S A 105, 10396-401 (2008).

20. Buchwald, G. et al. Structure and E3-ligase activity of the Ring-Ring complex of polycomb proteins Bmi1 and Ring1b. Embo J 25, 2465-74 (2006). 
Summary 


\section{Summary}

All genetic information that a cell needs to function is stored in its DNA. Genes are transcribed into mRNA, which is subsequently translated into proteins. When a cell prepares for cell division, the nuclear DNA is duplicated and divided between two daughter cells and all genetic information is maintained. During development, cells acquire specific functions, mediated by gene-environment interactions. Resting stem or progenitor cells are activated by molecules in their direct environment. These signals are transduced to the nucleus, where, depending on the nature of the signal(s), specific genes are activated, resulting in the production of RNA's (mRNA, non-coding RNAs) and proteins which trigger a differentiation program, that ultimately leads to formation of specialized, fully functional cells.

Although most somatic cells in a human body contain identical DNA sequences, the vast variety of cellular phenotypes implies that the information stored in the DNA sequence on its own is not sufficient to explain cell diversity and function. An additional layer of regulation, other then the DNA sequence itself, regulates accessibility of the DNA template. Nuclear DNA is not 'naked' but packed into a complex structure called chromatin. Chromatin predominantly consists of DNA wrapped around histone proteins. Histone proteins can be posttranslationally modified, and via these modifications many different protein complexes can bind and regulate chromatin. Chromatin structure is found in several 'states', ranging form tightly condensed inaccessible chromatin to open and accessible chromatin. Processes like DNA duplication (replication) and gene activation (transcription) are regulated by changes in chromatin structure, via increased or decreased accessibility of the DNA template. Collectively, these processes, that may involve concerted action of chemically modified DNA and proteins and non-coding RNAs, are referred to as epigenetic regulation. Polycomb group proteins $(\mathrm{PcG})$ have been identified as epigenetic regulators of chromatin structure, and are best known as transcriptional regulators of Hox genes. An overview of our current understanding of regulation of replication and transcription by epigenetics in general and by PcG proteins in particular is presented in chapter 2 .

Although insight into Polycomb biology has improved a lot over recent years, mechanisms of action of PcG proteins are far from fully understood. Main questions are What is the mechanism behind PcG-mediated transcriptional repression? Is there perhaps a role of PcG in replication and DNA repair? How are PcG proteins involved in mediating gene-environment interactions? How are PcG proteins regulated themselves? How are PcG proteins targeted to lineage-type specific gene targets?

As PcG proteins are involved in antero-posterior axis formation and many single PcG mutants cause skeletal abnormalities, this thesis aimed at elucidating the role of PcG proteins in one important aspect of enchondral ossification, namely chondrogenic differentiation. 
The formation of bone (enchondral ossification) is a multistep process, in which an extracellular matrix is produced by chondrocytes that is ultimately calcified by osteocytes. Numerous locally produced soluble factors regulate different stages in this process, strongly suggesting that the microenvironment plays an important role in enchondral ossification. Many of these factors are activated in response to hypoxia, and downstream of HIF1a activation, which is consistent with the absence of blood vessels in cartilage. Most of these findings result from in vitro studies, and currently studies on enchondral ossification often are performed on whole embryos or growth plates. Chapter 3 describes the molecular analysis of pathways relevant to enchondral ossification in a novel in vivo model in rabbits. The model makes use of experimentally induced local damage of periosteum, which results in activation of mesenchymal stem cell (MSC)-like activity, and periosteal callus formation. Subsequently the callus is used to functionally restore an experimentally induced articular cartilage lesion. Analysis of mRNA and protein expression in the repaired lesion confirms that the model recapitulates the sequential steps of normal chondrogenic development. In addition, we provide in vivo evidence that during the chondrogenic phase Hif1a is activated, suggesting that the conditions of periosteal callus formation are, at least transiently, hypoxic.

During cellular differentiation cells receive extracellular signals that lead to changes of the transcriptome (all cellular RNAs produced by transcription of DNA), resulting in production of RNAs and proteins needed for a specific cell type to adapt and function. At the onset of this project knowledge on Immediate Early Genes (IEGs) in chondrogenic differentiation was limited. The identification of IEGs as strong responders to a changing microenvironment (inducing chondrogenic differentiation), prompted us to study the role of the IEG Egr1 during ATDC5 chondrogenesis (chapter 4). In a loss-of-function approach, using short hairpin RNAs targeting Egr1 mRNA, Egr1 was found to function in at least two, possibly interconnected, processes: Egr1 is required for transcriptional activation of downstream chondrogenic differentiation pathways and Egr1 controls initiation of crucial epigenome wide reprogramming during chondrogenesis. Additionally, Egr1-LOF cells accumulate DNA damage, activate DNA damage responses, and induce a senescence-like response, which may be related to abnormal epigenetic responses. Relevantly, chapter 4 describes two ways in which Egr1 may connect to PcG biology.

Polycomb repressive complex 1 (PRC1) mutant mice display abnormalities in skeletal development that correlate to defective maintenance of HOX gene expression boundaries, but our understanding of epigenetic mechanisms underlying chondrogenic differentiation is poor. To gain more insight in molecular mechanisms mediated by PRC1 during chondrogenesis, we studied Bmi1 in ATDC5 chondrogenic differentiation (chapter 5). Upon induction of differentiation ATDC5 cells undergo a transient phase of hyperproliferation. Loss of Bmi1 leaves the cells incapable of undergoing this expansion phase. Bmi1-LOF cells accumulate massive amounts of DNA damage, 
arrest in S-phase, and consequently display characteristics of replicative senescence. Accumulation of DNA damage and activation of intra-S-phase checkpoints is associated with replication stress, collisions of replication and transcription machineries. This led us to study global localization of transcription and replication. Interestingly, control cells showed spatially separated regulation of these two DNA-based processes, whereas this coordinated regulation is lost in Bmi1LOF cells during rapid proliferation. Furthermore, DNA damage in Bmi1-deficient cells accumulates at sites of de novo DNA synthesis, suggesting that loss of PRC1 function during hyperproliferation renders the cells unable to deal with increased chromatin stress. The data presented in chapter 5 reveals an important function for PRC1 in orchestrating simultaneous chromatin-associated processes, i.e. DNA replication and transcription, extending PRC1 function as merely transcriptional repressors.

Valproic acid (VPA) is a drug used to treat epilepsy and other neurological conditions. VPA administration during pregnancy can lead to neural tube defects, skeletal transformations and other adverse effects in the unborn child. VPA was designated as an epigenetic drug, but molecular mechanisms of actions are unclear. Treatment of differentiating ATDC5 cells with VPA in therapeutically relevant concentrations completely abrogates the ability of chondrogenic progenitors to differentiate, which is accompanied by severe alterations in global epigenetic modifications. Additionally, VPA affects the differentiation-induced hyperproliferation in a TP53dependent manner. Completely in line with the data presented in chapters 4 and 5, VPA treated cells accumulate replication stress, DNA damage, and induce senescence-like responses, further strengthening the conclusions that epigenomic remodeling is critical for proper differentiation to occur (chapter 6).

Taken together, data in this thesis shows that in response to extracellular differentiation stimuli, epigenomic remodeling allows cells to and tune DNA-templated processes (transcription, replication), under conditions of increased proliferation and RNA and protein production. When epigenomic reprogramming fails, combined stress of uncoordinated transcription and replication stress leaves cells incapable of undergoing chondrogenic differentiation. The significance of the results in this thesis is discussed in chapter 7. 
Samenvatting 


\section{Samenvatting}

Alle genetische informatie die nodig is om een cel te laten functioneren ligt opgeslagen in 'n DNA. Genen kunnen afgeschreven worden in mRNA dat vervolgens kan worden vertaald naar eiwiten. Wanneer een cel zich voorbereidt voor celdeling, wordt het DNA in de celkern gedupliceerd en wordt het verdeeld tussen de twee dochtercellen, waardoor alle genetische informatie behouden blijft. Tijdens ontwikkeling, verkrijgen cellen een specifieke functie. Dit proces wordt gemedieerd door gen-omgevings interacties. Rustende stam of progenitor cellen worden geactiveerd door moleculen in hun directe omgeving. Deze signalen worden doorgegeven naar de celkern waar, afhankelijk van de aard van de signalen, specifieke genen worden geactiveerd, resulterend in de productie van RNA (mRNA, niet coderende RNA's) en eiwitten, die een differentiatie programma kunnen activeren, wat uiteindelijk leidt tot het ontstaan van gespecialiseerde, volledig functionele cellen. Hoewel bijna alle lichaamscellen in een menselijk lichaam identieke DNA sequenties bevat, impliceert de grote variatie aan gespecialiseerde cellen, dat de informatie die in het DNA ligt opgeslagen niet voldoende is om celdiversiteit en functie te verklaren. Een andere vorm van regulatie, naast de DNA sequentie zelf, reguleert toegangelijkheid van DNA. DNA in de kern is ingepakt in een compleze structuur, dat chromatine genoemd wordt. Chromatine bestaat voornamelijk uit DNA dat om histon eiwitten gewikkeld is. Histon eiwitten kunnen posttranslationeel gemodificeerd worden, en via deze modificaties kunnen vele verschillende eiwitcomplexen het chromatine binden en het reguleren. De structuur van chromatine bevindt zich in verschillende staten, variërend van een hechte, gecondenseerde, ontoegangkelijke staat tot een open en toegankelijke staat. Cellulaire processen als DNA duplicatie (replicatie) en gen activatie (transcriptie) worden gereguleerd door veranderingen in chromatine structuur, via verhoogde of verlaagde toegankelijkheid van het DNA. Chromatine structuur wordt onder meer gereguleerd door chemische modificaties van DNA en eiwitten, en niet-coderende RNA. Samen wordt deze vorm van regulatie epigenetica genoemd. Polycomb groep eiwitten (PcG) zijn geindentificeerd als epigenetische regulatoren van chromatine structuur, en zijn vooral bekend als transcriptionele regulatoren van Hox genen. Een overzicht van ons huidige begrip van regulatie van transcriptie en replicatie door epigenetica in het algemeen en specifiek door PcG eiwitten wordt beschreven in Hoofstuk 2.

Hoewel het inzicht in Polycomb biologie flink is toegenomen de afgelopen jaren, worden de mechanismen van PcG functioneren nog maar voor een klein deel begrepen. Er zijn in dit vakgebied verschillende kernvragen. Wat is het mechanisme van PCG gemedieerde transcriptionele repressie? Is er wellicht een rol voor PcG in replicatie en DNA reparatie? Hoe zijn PcG eiwitten betrokken bij het mediëren van gen-omgevings interacties? Hoe worden PcG eiwitten zelf gereguleerd? Hoe worden PcG eiwitten naar celspecifieke genen gestuurd? 
Aangezien PcG eiwitten betrokken zijn bij antero-posterior as vorming en PcG mutante muizen skelet afwijkingen hebben, richt deze thesis zich op het verhelderen van de rol van PcG eiwitten in een belangrijk aspect van enchondrale ossificatie, namelijk chondrogene differentiatie.

De vorming van bot (enchondrale ossificatie) is een meerstaps proces, waarin een extracellulaire matrix gevormd wordt door chondrocyten (kraakbeencellen), die uiteindelijk verkalkt wordt door osteocyten (botcellen). Talloze lokaal geproduceerde oplosbare factoren reguleren de verschillende stadia van deze processen, wat aangeeft dat de directe omgeving een sterke rol speelt in enchondrale ossificatie. Veel van deze factoren worden geactiveerd in reactie op een hypoxische omgeving, en activatie van de transcriptie factor HIF1a, wat consistent is met de afwezigheid van bloedvaten in kraakbeen. Het overgrote deel van deze bevindingen komen voort uit in vitro studies. Daarnaast worden vele studies naar enchondrale ossificatie uitgevoerd op totale embryos of groeiplaten. Hoofdstuk 3 beschrijft de moleculaire analyse van relevante signaleringspaden tijdens enchondrale ossificatie in een nieuw in vivo model in konijnen. Dit model maakt gebruik van een lokaal geinduceerde beschadiging van het periosteum, wat resulteert in de activatie van mesenchymale stem cell (MSC) gelijkende activiteit, en periosteale callusvorming. Vervolgens wordt de callus gebruikt voor een functioneel herstel van een experimenteel geinduceerde articulaire kraakbeen lesie. Analyse van mRNA en eiwit expressie bevestigt dat het model de opvolgende stappen van normale chrondrogene ontwikkeling recapituleert. Daarnaast, bieden we in vivo bewijs voor activatie van HIF1a tijdens de chondrogene fase, wat suggereert dat de omstandigheden van periosteale callusvorming, op zijn minst transient, hypoxisch zijn.

Tijdens cellulaire differentiatie ontvangen cellen extracellulaire signaken die leiden tot veranderingen in het transcriptoom (alle RNA's die tijdens transcriptie van DNA geproduceerd worden), resulterend in productie van specifieke RNA's en eiwitten die een cel nodig heeft om zich aan te passen en een nieuwe functie aan te nemen. Aan het begin van dit project was er nauwelijks kennis van de rol van Immediate Early Genes (IEG's) tijdens chondrogene differentiatie. De ontdekking dat IEG's zeer sterk reageren op een veranderende omgeving, leidde tot het bestuderen van de rol van de IEG Egr1 tijdens chondrogene differentiatie in ATDC5 cellen (hoofdstuk 4). In een verlies van functie aanpak, gebruik makend van geinduceerde afbraak van Egr1 mRNA, bleek Egr1 een tweeledige rol te spelen, die mogelijk verband met elkaar houden. Egr1 is vereist voor transcriptionele activatie van chondrogene differeniatie signaleringspaden, en ook initieert Egr1 cruciale epigenoom wijde herprogrammering tijdens chondrogenese. Daarnaast lopen cellen zonder Egr1 tijdens chondrogenese DNA schade op, activeren ze DNA schade responsen, en induceren ze een verouderingsresponse (senescentie), die vermoedelijk gerelateerd is aan abnormale epigentische responsen. Hoofdstuk 4 beschrijft twee manieren waarin Egr1 vermoedelijk gelinkt aan PcG biologie is. 
Polycomb repressive complex 1 (PRC1) mutante muizen vertonen abnormale skelet ontwikkeling, die correleert met defecte regulatie van Hox gene expressie, maar ons begrip van epigenetische mechanismen die hieraan ten grondslag liggen is beperkt. Om meer inzicht te krijgen in de moleculaire mechanismen die door PRC1 gemedieerd worden tijdens chondrogenese, bestudeerden we Bmi1 in ATDC5 chondrogene differentiatie (hoofdstuk 5). In response op inductie van differentiatie ondergaan ATDC5 cellen een transiente fase van hyperproliferatie. Verlies van Bmi1 functie leidt ertoe dat de cellen deze expansie fase niet kunnen ondergaan. Zonder Bmi1 lopen de cellen enorme hoeveelheden DNA schade op, ondergaan ze een S-fase arrest, en laten ze vervolgens karakteristieken zien van replicatieve senescentie. Opstapeling van DNA schade en activatie van een S-fase arrest wordt geassocieerd met replicatie stress, collissies van replicatie en transcriptie machineriën. Hierom bestudeerden we globale lokalisatie van transcriptie en replicatie. Controle cellen lieten een ruimtelijke scheiding zien van deze twee processen, terwijl deze gecoördineerde regulatie verloren is gegaan in cellen zonder Bmi1, die deze fase van hyperproleratie ondergaan. Daarnaast lopen de Bmi1 deficiënte cellen DNA schade op op posities waar replicatie plaatsvindt, wat suggereert dat cellen met verlies van PRC1 functie tijdens hyperproliferatie niet in staat zijn om met verhoogde chromatin stress om te gaan. De data in hoofdstuk 5 laat zien dat PRC1 een belangrijke rol speelt in het regisseren van chromatine geassocieerde processen, zoals replicatie en transcriptie, wat betekent dat PRC1 niet enkel functioneert als transcriptionele repressor.

Valproaat is (VPA) is een medicijn dat wordt gebruikt om epilepsie en andere neurologische aandoeningen te behandelen. VPA gebruik tijdens zwangerschap kan leiden tot neurale buis defecten, malformaties van het skelet, en andere aandoeningen bij het ongeboren kind. VPA is beschreven als een epigenetisch medicijn, maar de moleculaire effecten zijn onbekend. Behandeling van differentierende ATDC5 cellen met therapeutisch relevante concentraties VPA leidt ertoe dat ATDC5 cellen het vermogen om te differentieren verliezen. Dit gaat gepaard met zeer sterke veranderingen in gobale epigenetische modificaties. Daarnaast, verliezen deze cellen de capaciteit om te hyperproliferen in een TP53 afhankelijke manier. Totaal in lijn met de experimenten beschreven in hoofdstukken 4 en 5, lopen de VPA behandelde cellen replicatie stress en DNA schade op. Eveneens activeren deze cellen senescentie responsen, wat verder bijdraagt aan de conclusies dat epigenomische herprogrammering essentieel is om volledige differentiatie plaats te laten vinden (hoofdstuk 6).

De data in dit proefschrift laat zien dat in response op extracellulaire differentiatie stimuli, epigenomische herprogrammering de cellen in staat stelt om DNA gebaseerde processen (o.a. transcriptie en replicatie) op elkaar af te stemmen tijdens omstandigheden van versnelde proliferatie, en RNA en eiwit productie. Wanneer epigenomische herprogrammering niet goed plaats vindt, verliezen cellen het vermogen om chondrogeen te differentiëren, vanwege replicatie stress. De significatie van de resultaten beschreven in dit proefschrift worden bediscussieerd in hoofdstuk 7. 


\section{Dankwoord}




\section{Dankwoord}

Eindelijk is het dan zover, "het boekje" is af! Terugdenkend aan deze periode realiseer je je pas met hoeveel mensen je hebt samengewerkt. Jullie hebben er mede voor gezorgd dat dit proefschrift tot stand is gekomen, maar ook dat ik altijd met plezier in Maastricht heb gewerkt. Ook zijn er zoveel mensen geweest die interesse en enthousiasme getoond hebben, waardoor het op momenten dat het even tegen zit makkelijker is om toch nog even door te zetten. Daarom wil ik nu graag het woord tot jullie richten.

Allereerst mijn co-promoter en promoter.

Willem, toen ik een aantal jaren terug bij jou binnenstapte en een stortvloed aan informatie kreeg over PcG's realiseerde ik me dat je als vers afgestudeerde eigenlijk pas begint met kennis opdoen. Wat dat betreft kan ik me geen betere leermeester voorstellen. Jouw enthousiasme voor wetenschap en het vermogen om dit op anderen over te brengen is een echte motivatie. Daarnaast heeft jouw brede parate kennis en inzicht in wetenschap ertoe geleid dat het woord serendipiteit (beeldend omschreven door de nederlandse Nobelprijs winnaar Pek van Andel als het zoeken naar een naald in een hooiberg, en eruit komen met een boerenmeid :) verschillende keren de revue passeerde, en we onverwachte data snel konden herkennen en in de juiste context konden plaatsen. De tijd van oogsten is gekomen, en met al de ideeën voor experimenten die we nog hebben, gaan we nog een spannende tijd tegemoet! Willem, ontzettend bedankt!!

Professor Geraedts, beste Joep, uw rol heeft zich vooral achter de schermen afgespeeld, maar dat maakt die rol zeker niet minder belangrijk. Hartelijk bedankt voor uw inzet.

Graag wil ik de beoordelings commissie onder leiding van Prof. Dr. F.C.S. Ramaekers danken voor de kostbare tijd en het beoordelen van dit proefschrift. Ik kijk er naar uit "de degens te kruisen".

Dan de WVO groep! Dankzij jullie zijn de afgelopen jaren omgevlogen! De saamhorigheid werkt motiverend, is bijzonder prettig en zeker ook gezellig. Hanneke (Hannniiieee!), je was een paar maanden eerder begonnen dan ik, waardoor ik veel van je heb kunnen leren in de begin periode. Al die uren op het lab zijn een stuk sneller gegaan door samen met de radio mee te zingen, al ligt onze muzikale interesse behoorlijk ver uit elkaar. Doordat je een paar maanden voor me uit liep, heb ik flink van je kunnen profiteren de laatste tijd, dus veel dank voor de duwtjes in de goede richting! Céline, you were such a pleasant colleague. You found your way back to France, and I wish you and your family all the best! Vivian, rustig en hardwerkend, ben je altijd bereid om vragen te beantwoorden en dingen uit te zoeken. Van 
jouw vermogen om efficient en nauwkeurig een hoop tegelijk te doen, kunnen veel mensen wat leren! Peggy (oftewel Pegsywegsy, ofwel Babbelbox), wat een gezelligheid, altijd wel wat te vertellen. Je gaat inmiddels ook al weer bijna de afsluitende periodein, waar ik je veel succes bij wens. Gelukkig ben ik er nog om je hierbij te steunen. Guus, als student heb ik je alles kunnen vertellen wat ik weet over de ATDC's. Je hebt dit allemaal snel opgepikt, wat er mede toe heeft geleid dat je mocht blijven :P en we samen de verbinding met Orthopedie vormen. Verder Claudia, Raymond (een van weinigen die mijn koffiegebruik kan evenaren...), Juliette, Nard, Iris en Elena bedankt voor jullie meer of mindere bijdrage aan dit proefschrift! Ook alle studenten (Yvette, Marjolein, Rene, Caroline, Martijn, Sjoerd, Joyce, Chantal, Frans, en vele anderen!) die voorbij zijn gekomen wil ik bedanken voor hun bijdrage, zowel voor hun werk op het lab, als het bijdragen aan gezelligheid en het groepsgevoel.

Dan mijn orthopedische vrienden. Samen met Iris namen we de eerste stappen naar een inmiddels zeer hechte samenwerking. De groep is met de jaren flink toegenomen, maar het enthousiasme dat we van jullie kant mogen ontvangen is een constante factor. Tim, met jouw moleculair biologische achtergrond en drive, ben je inmiddels van grote waarde als verbindende factor tussen de twee groepen. Het samen begeleiden van enkele studenten heeft er toch mooi voor gezorgd dat ze goed terecht zijn gekomen (namelijk binnen onze samenwerking!). Don, als partner in crime van het eerste uur gaat er veel dank uit naar jou, uiteraard ook omdat je me na al die jaren aan het tennissen hebt gekregen! Andy, hartstikke bedankt voor bijdragen aan het VPA verhaal! Pieter, al zien we je niet veel vanwege jouw klinische verplichtingen, bedankt dat ik ook mocht bijdragen aan jouw onderzoek. Marjolein, als student ben je begonnen binnen deze samenwerking. Je hebt een hoop werk verricht aan het Egr1 hoofdstuk, en je bent inmiddels een eind op weg met je eigen promotietraject met veel mooie data, veel succes daarbij.

De afgelopen jaren hebben we bij Moleculaire Genetica een hoop mensen zien komen en gaan. De grote variatie in onderzoek op de afdeling heeft ertoe geleid dat er niet veel is samengewerkt met de verschillende groepen. Een voordeel hiervan is wel dat er in de verschillende groepen een diversiteit aan labtechnische kennis is. Hierbij wil ik van de mogelijkheid gebruik maken om alle mensen die hebben bijgedragen te bedanken. Mijn (oud-)kamergenoten Sofia (also for the gezelligheid outside of work!), Debby (Koonen the Barbarian), Susan, Laura, Yeliz en Wino. Marten (voor de bijdragen tijdens de werkbesprekingen en mijn aio commissie bijeenkomsten), Ronit (for critically reviewing the Bmi1 paper and the review), Patrick (voor het 1000 en 1 keer helpen met van alles en nog wat!), Veerle B, Kristiaan en Ellen L, Jana, Menno, Monique, Marjon (zodat ik aan mijn 
maatschappelijke verplichtingen kon voldoen, en er achter kwam dat ik toch niet in de wieg ben gelegd als voetbaltrainer), Danielle $C$ (oa voor de begeleiding tijdens mijn afstuderen bij GRAT), Le (for liking my gehaktballen and the chitchat. See you in London!), Monique, Inge, Chantal, Stijn, Matthijs, Remco, Pieter, Lauran, Nadine, Veerle C, Guillaume, Sander en Petra, Jan, Joost (voor het delen van mijn interesse in AJAX, en altijd een, en dan bedoel ik 1 en hetzelfde, onderwerp, aan te kaarten om te kletsen:), Robert (mijn gezellige nieuwe kamergenoot), Daphna, Ellen D, Ine, Marjo, Nicole, Will, Mohammed, Danielle vd V, Maurice, Wilma, Brigitte, Roy, Olivier en Arjen. Allen bedankt!

Dan zijn er nog een hoop mensen van andere afdelingen. Van Moleculaire Celbiologie, Andrea (for being a good friend, letting me win with squash, and for showing interest), Bert $D$ (je hebt altijd je woordje klaar, of het nou gaat om bijhand te zijn, of om technische adviezen), Mieke en Bert S. voor techische adviezen en BrdU antilichamen. Verder, de mensen van Maastro en Pulmulogie voor hun bijdragen in de werkbesprekingen en journalclubs, waardoor we een breder blik op wetenschap kregen dan alleen PcG eiwitjes. Brad, Marianne, Jan, Roland, Kasper, Twan, Chantal, Ludwig, Michael, Maud, Sherry, Ashferd, Kim, Kim, Natasja en Younan van Maastro, en van Pulmunologie natuurlijk Jos (ook voor de nodige uurtjes zweten in de squashzaal en de wetenschappelijke praatjes tijdens het uitpuffen) en Ramon onder andere voor het deelnemen in de beoordelingscommissie.

Vrienden en familie. De afgelopen jaren hebben veel mensen interesse getoond in mij en mijn onderzoeken. Aangezien dit teveel mensen zijn om iedereen persoonlijk te noemen: allen bedankt! Toch wil ik een aantal mensen persoonlijk noemen. Uiteraard mijn paranymfen, Joris en Eric. We gaan inmiddels een lange tijd terug. We hebben erg veel meegemaakt, en het is toch mooi om te zien dat we sinds de Einsteinstraat alle drie zo'n eind gekomen zijn. Ik ben er trots op dat jullie mij straks bij de verdediging als paranymf zullen bijstaan! Carmen, had je ooit kunnen denken dat er, toen je Eric leerde kennen, nog een verrassing in het pakket zat?? Je bent de afgelopen jaren een goeie vriendin geworden, en ik wil je ook bedanken dat Eric en ik de kans krijgen om onze vriendschap ten volle te benutten. Tom en Gemma, helaas kunnen jullie niet bij mijn promotie zijn, maar het goede werk dat jullie nu doen in Colombia is een inspiratie.

En tenslotte, pap en mam. Tja, woorden zijn niet genoeg om mijn dankbaarheid te uiten. De stabiele thuisbasis heeft Tom en mij veel gebracht. Niet alleen een goede jeugd, maar ook de mogelijkheid om ons op allerlei terreinen te ontwikkelen, qua hobby's en opleiding, maar zeker ook als mens. Hoewel de afstand af en toe wat groot is zijn we de afgelopen jaren nog meer naar elkaar toe gegroeid, en zonder jullie steun en vertrouwen had ik het nooit gered. Pap en mam, deze is voor jullie! 
Curriculum Vitae 
Frank Spaapen werd geboren op 7 februari 1978 te Haarlem en groeide op in Vogelenzang. In 1990 rondde hij het VWO af op het Sancta Maria te Haarlem. In datzelfde jaar begon hij de studie Gezondheidswetenschappen aan de universiteit te Maastricht. Na gekozen te hebben voor het moleculair biologisch traject, voerde hij een korte stage uit op de afdeling Moleculaire Celbiologie onder supervisie van Bert Schutte. Tijdens deze stage werd klieving van cytokeratines tijdens inductie van apoptose bestudeerd. Tijdens zijn afstudeerstage aan de afdeling Gezondheidsrisico analyse en toxicologie werkte bij aan studies naar de genotoxische effecten van carcinogenen in humane longepitheelcellen. Deze stage was onder begeleiding van Prof. Dr. F.J. van Schooten en Danielle Curfs, en werd met succes afgerond waardoor hij in februari 2003 zijn diploma mocht ontvangen. Direct aansluitend op het afstuderen begon hij zijn werkzaamheden in de onderzoeksgroep van Dr. J.W. Voncken in de capaciteitsgroep Moleculaire Genetica aan de Universiteit Maastricht waar zijn interesse in moleculaire biologie zeer goed van pas kwam. Na een aantal maanden als analyst te hebben gewerkt, begon hij zijn promotieonderzoek naar de rol van PcG eiwitten tijdens chondrogene differentiatie. Na het afronden van het promotieonderzoek blijft hij werkzaam in de onderzoeksgroep van Dr. J.W. Voncken waar hij zich richt op de rol van PcG eiwitten tijdens replicatie timing. Door de toekenning van een EMBO fellowship wordt hij in staat gesteld om bekend te raken met de technieken om replicatie timing te bestuderen onder begeleiding van Dr. V. Azuara op het Imperial College te Londen. 


\section{List of publications}


Pieter J. Emans, Lodewijk W. van Rhijn, Tim J.M. Welting, Andy Cremers, Nina Wijnands, Frank Spaapen, J. Willem Voncken, V. Prasad Shastri. Autologous Engineering of Cartilage. In press, PNAS. (IF: 9.38)

Remels AHV, Langen RCJ, Gosker HR, Russell AP, Spaapen F, Voncken JW, Schrauwen P, Schols AMWJ. PPAR-y inhibits NF-kB-dependent transcriptional activation in skeletal muscle. Am J Physiol Endocrinol. (IF: 3.324)

Pieter J. Emans, Frank Spaapen, Don A.M. Surtel, Keryn M. Reilly, Andy Cremers, Lodewijk W. van Rhijn, Sjoerd K. Bulstra, Jan Willem Voncken, Roel Kuijer; A novel in vivo model to study endochondral bone formation; HIF-1alpha activation and BMP expression. Bone. 2007 Feb; 40(2):409-18. (IF: 4.145)

Spaapen F, Eijssen L, Welting T, Salvaing J, Prickaerts P, Dahlmans V, Surtel DAM, Koseki H, Vidal M, Takihara Y, Wouters B, Voncken JW.

Polycomb Repressive Complex 1 Coordinates Enhanced Replication and Transcription in Transit Amplifying Chondrogenic Progenitors. In preparation

Frank Spaapen, Guus van den Akker, Marjolein Caron, Peggy Prickaerts, Hanneke Niessen, Vivian Dahlmans, Don A.M. Surtel, Yvette Paulis, Finja Schweizer, Celine Rofel, Tim Welting, Lars Eijssen, Jan Willem Voncken. The immediate early gene EGR1 controls epigenetic reprogramming through Polycomb Group proteins during chondrogenesis. In preparation

Frank Spaapen, Vivian Dahlmans, Andy Creemers, Pieter Emans, Tim Welting, J. Willem Voncken.

Proliferative Capacity Defines Sensitivity to Valproic Acid During Chondrogenesis. In preparation

F. Spaapen, Y. Takihara, H. Brock, J.W. Voncken. Replicating DNA at Top Speed- Chromatin at Risk. (New tales from aging bones). Review, in preparation

Niessen HEC, Prickaerts P, Salvaing J, Spaapen F, Dahlmans V, Geijselaers V, Bartels S, Takihara Y, Hansen K, Wouters B, Bracken AP, Rapp UR and Voncken JW. MK3 controls proliferation by an epigenetic switch-mechanism involving Polycomb function. Submitted.

Niessen HEC, Dahlmans V,Prickaerts P, Spaapen F, Geijselaers C, Lee S-C, Wouters BG, Demmers $\mathrm{J}$ and Voncken JW. Cell stress-induced association of Polycomb Repressive Complex 1 with the chromatin factor KAP1. In preparation. 
Abbreviations 


\begin{tabular}{|c|c|}
\hline ACAN & Aggrecan \\
\hline AP & Antero-posterior \\
\hline ATM & Ataxia telangiectasia mutated \\
\hline ATR & Ataxia telangiectasia mutated- and Rad3-related \\
\hline BARR & Barren \\
\hline BMI1 & B-cell-specific Moloney murine leukemia virus integration site 1 \\
\hline BMP & Bone morphogenetic protein \\
\hline BrdU & 5-bromo-2-deoxyuridine \\
\hline CBX & Chromobox protein \\
\hline ChIP & Chromatin immunoprecipitation \\
\hline Chk & Checkpoint kinase \\
\hline Col10a1 & Collagen 10a1 \\
\hline Col2a1 & Collagen 2a1 \\
\hline CRM & Cramped \\
\hline CryGS & yCrystalline \\
\hline DAPI & 4',6-diamidino-2-phenylindole \\
\hline DDR & DNA Damage Response \\
\hline DNMTs & DNA methyltransferases \\
\hline DSB & Double strand break \\
\hline DUB & Deubiquitylase \\
\hline e.g. & Exempli gratia \\
\hline $\mathrm{ECM}$ & Extracellular matrix \\
\hline EDR & Early developmental regulator \\
\hline Egr & Early growth response \\
\hline ERSS & Endoplasmatic reticulum stress signaling \\
\hline ESCs & Embryonal stem cells \\
\hline ETP & Enhancer of Trithorax and Polycomb \\
\hline ETP & Etoposide \\
\hline $\mathrm{EZH} 2$ & Enhancer of Zeste homologue \\
\hline FC & Fold change \\
\hline FDA & Food and Drug Administration \\
\hline GMNN & Geminin \\
\hline GTF & General transcription factor \\
\hline $\mathrm{H} 2 \mathrm{~A}$ & Histone 2A \\
\hline $\mathrm{H} 2 \mathrm{AX}$ & Histone $2 A x$ \\
\hline H3K27me3 & Histone 3 lysine 27 trimethylation \\
\hline H3K4 & Histone 3 lysine 4 \\
\hline H3K9me3 & Histone 3 lysine 9 trimethylation \\
\hline H3S10 & Histone 3 serine 10 \\
\hline H3S28 & Histone 3 serine 28 \\
\hline HAT & Histone acetyltransferase \\
\hline HDAC & Histone deacetylase \\
\hline HIF1a & Hypoxia inducible factor $1 \alpha$ \\
\hline HKMT & Histone lysine methyltransferase \\
\hline HMT & Histone methyltransferase \\
\hline $\mathrm{HZ}$ & Hypertrophic zone \\
\hline i.a. & Inter alia \\
\hline i.e. & id est \\
\hline IEG & Immediate early gene \\
\hline Ihh & Indian hedgehog \\
\hline Kap1 & KRAB-associated protein 1 \\
\hline KD & Knockdown \\
\hline LCR & Locus control region \\
\hline LOF & Loss of function \\
\hline MAPK & Mitogen activated protein kinase \\
\hline MCM & Mini chromosome maintenance \\
\hline
\end{tabular}




\begin{tabular}{ll} 
MSC & Mesenchymal stem cell \\
NSC & Neuronal stem cell \\
OIS & Oncogene induced senescence \\
ORC & Origin recognition complex \\
PC & Polycomb \\
PC & Perioseal callus \\
PCG & Polycomb Group \\
PCNA & Proliferating cell nuclear antigen \\
PH & Polyhomeotic \\
PHC & Polyhomeotic-like \\
PIC & Pre-initiation complex \\
PID & Post induction of differentiation \\
PO & Postoperative \\
POSTN & Periostin \\
PRC & Polycomb repressive complex \\
PRE & Polycomb responsive element \\
pre-RC & pre-Recognition complex \\
PTHrP & Parathyroid related protein \\
PTM & Posttranslational modification \\
RING & Really interesting new gene \\
RNF2 & Ring finger protein \\
ROS & Reactive oxygen species \\
RPOLII & RNA polymerase II \\
SAHF & Senescence associated heterochromatin formation \\
SASP & Senescence associated secretory pheontype \\
SA $\beta$ Gal & Senescence associated $\beta$ galactosidase \\
SCMH & Sexcombs on midleg homologue \\
shRNA & Short hairpin RNA \\
siRNA & Small interfering RNA \\
SUZ12 & Suppressor of Zeste 12 \\
TA & transit amplification \\
TBP & TATA-box binding protein \\
TFIIA & Transcription factor IIA \\
TFIIB & Transcription factor IIB \\
TFIID & Transcription factor IID \\
TGF $\beta$ & Transformation growth factor $\beta$ \\
Topo2 & Topoisomerase 2 \\
TRX & Trithorax \\
UPR & Unfolded protein response \\
VEGF & Vascular endothelial growth factor \\
VPA & Valproic acid \\
WT & Wildtype \\
\hline &
\end{tabular}


Abbreviations 
Colour pictures 


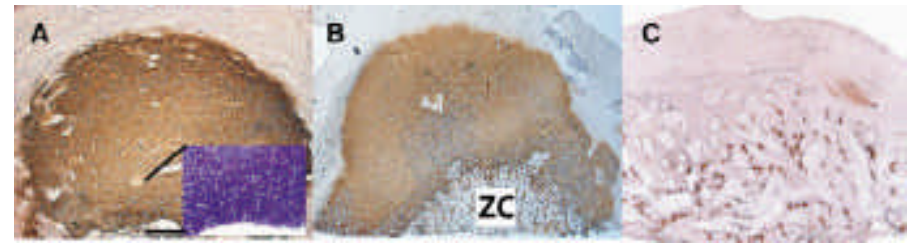

Fig.3.2 Collagen Type II in periosteal reactive tissue (callus)

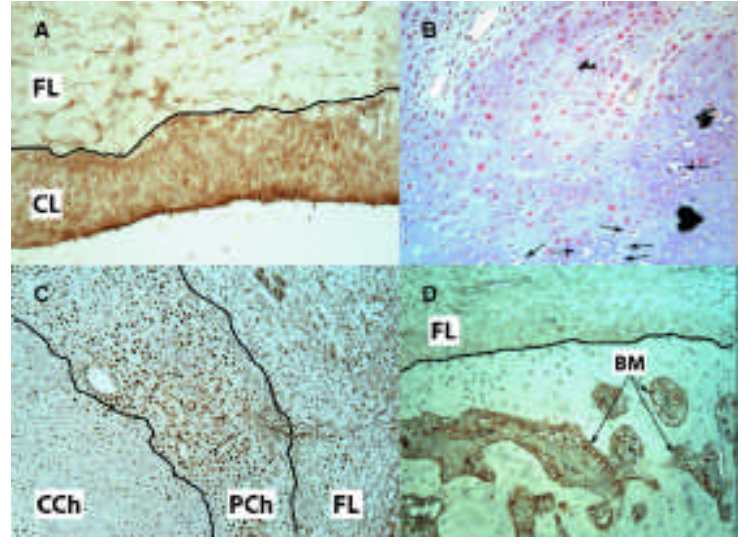

Fig. 3.3 Periostin/POSTN expression in periosteum

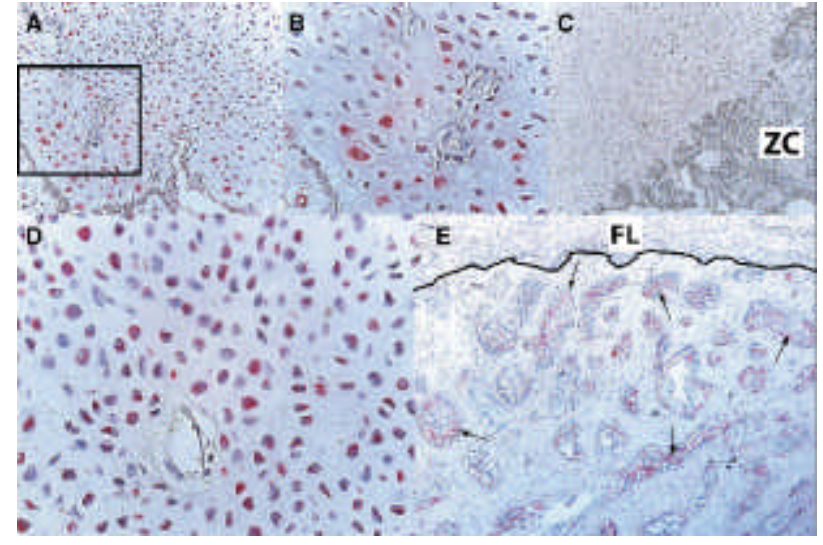

Fig 3.4 BMP-4 expression in periosteal callus
C
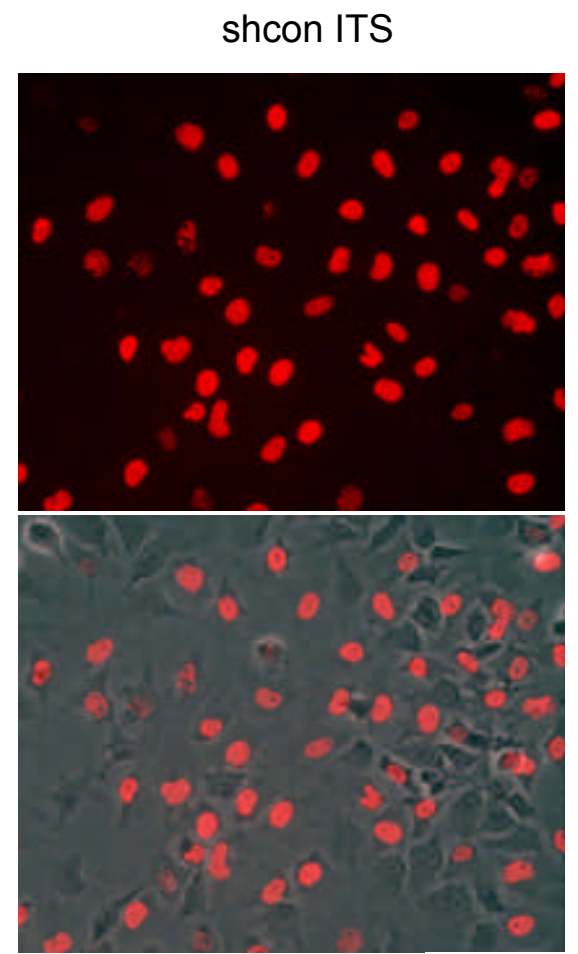

$\mathrm{t}=2$ pid (days)
shEGR1 ITS

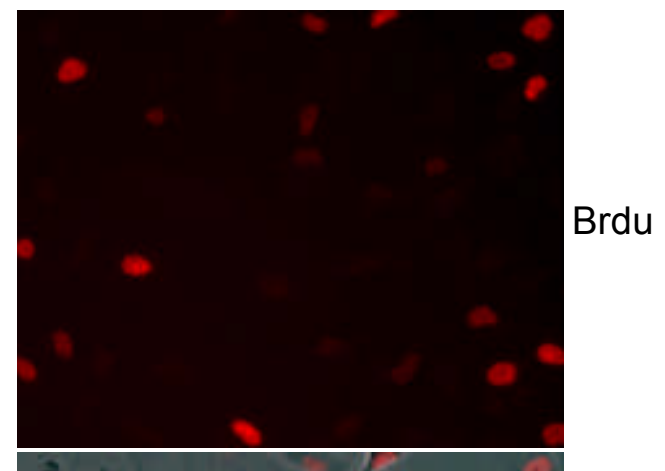

Merge phase

contrast

Fig 4.3 Egr1 is required for proper replication during chondrogenic replication 


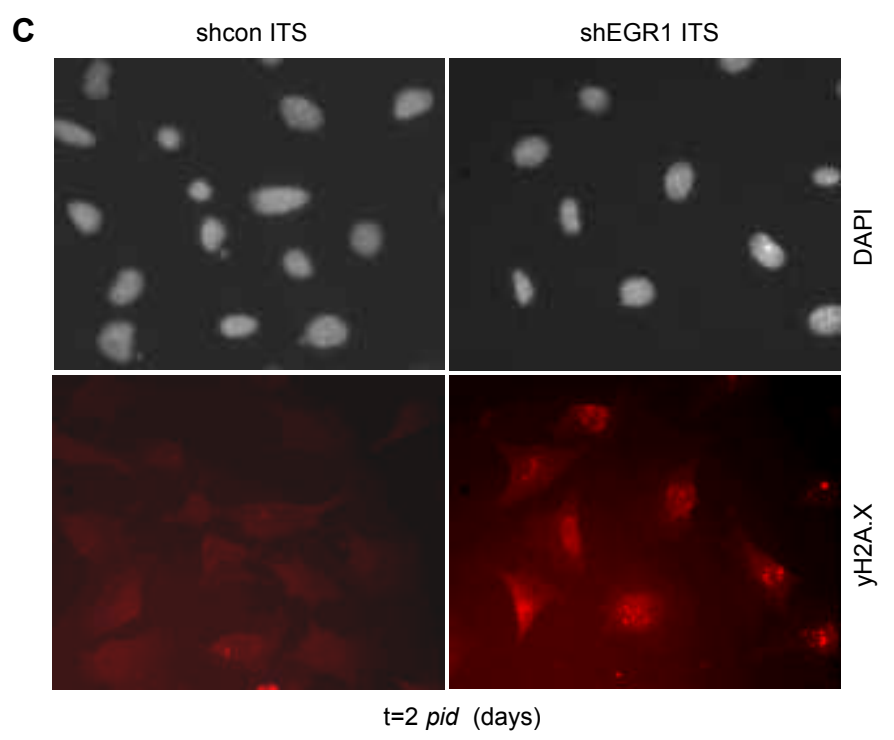

Fig 4.4c. Egr1-LOF accumulate DNA damage

C

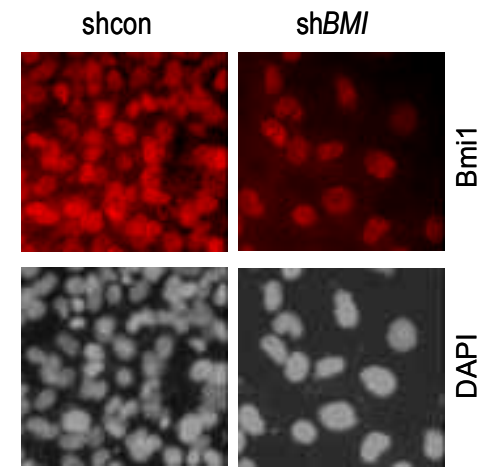

Fig 5.1c Bmi1 expression

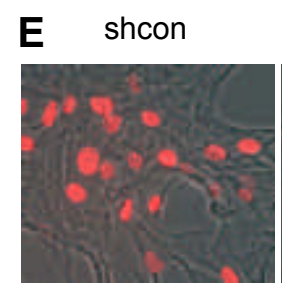

$\operatorname{sh} B M I$

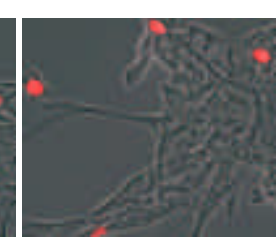

$\frac{\pi}{\sqrt{2}} \cdot \frac{\pi}{0}$

Fig 5.2e BrdU staining

\section{G}
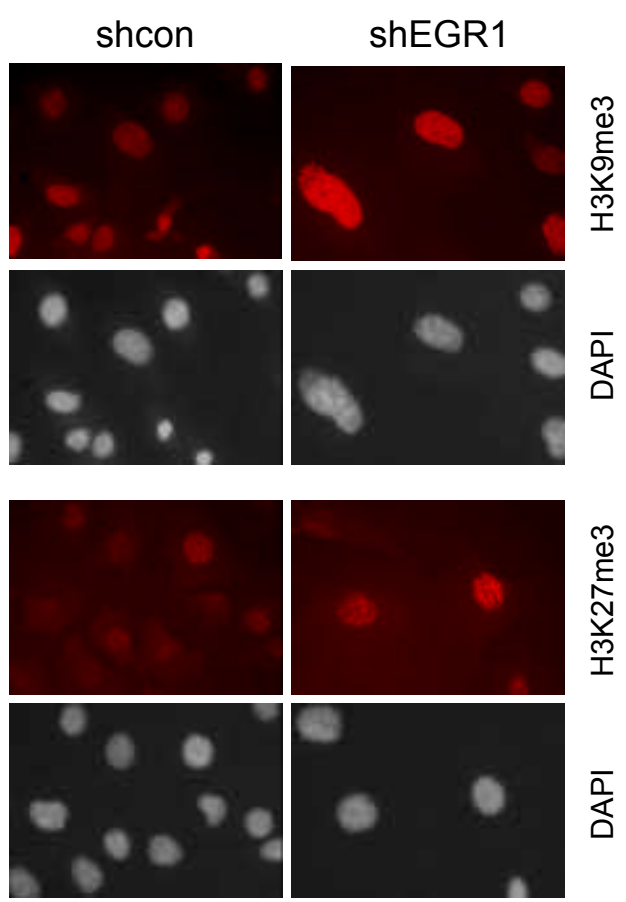

Fig $4.5 \mathrm{~g}$. Global histone modifications are affected in Egr1-LOF cells

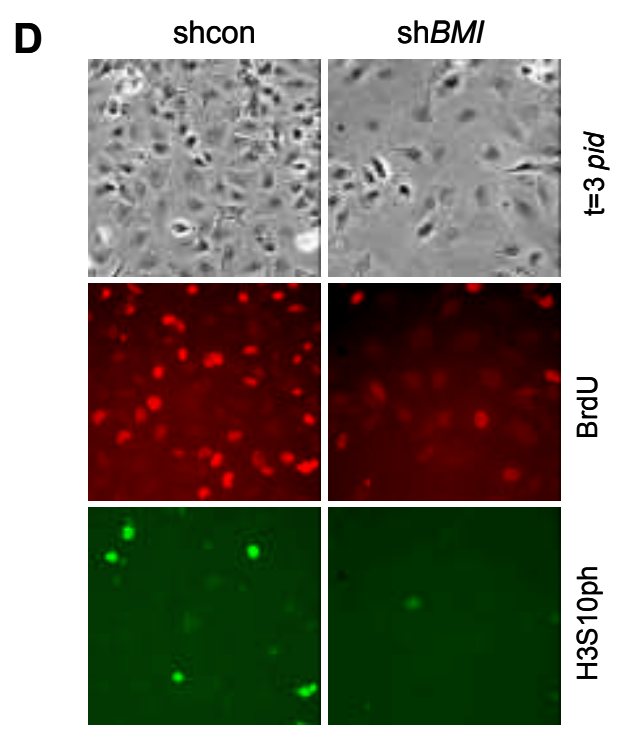

Fig 5.2d BrdU and H3S10ph staining 

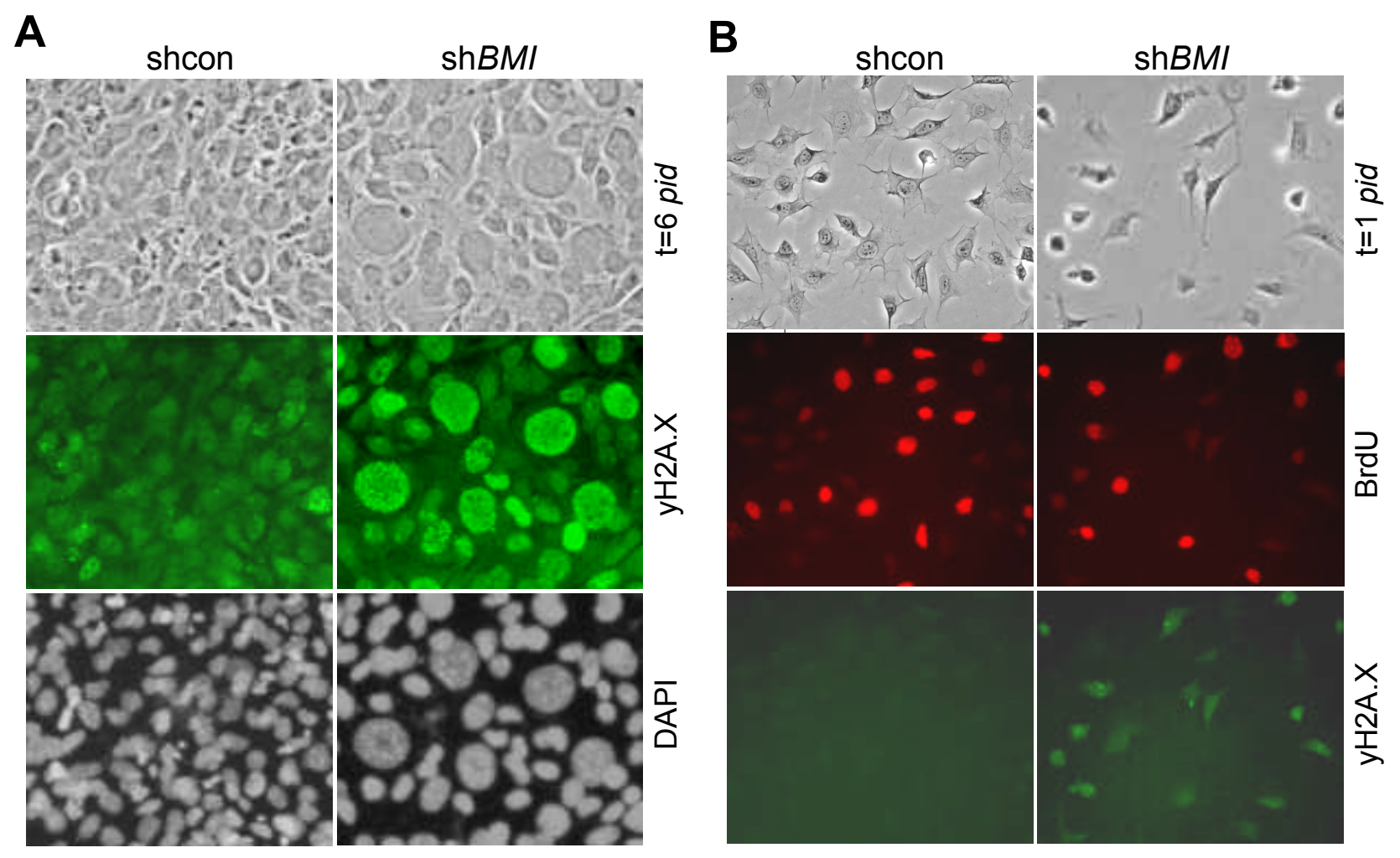

\section{D}

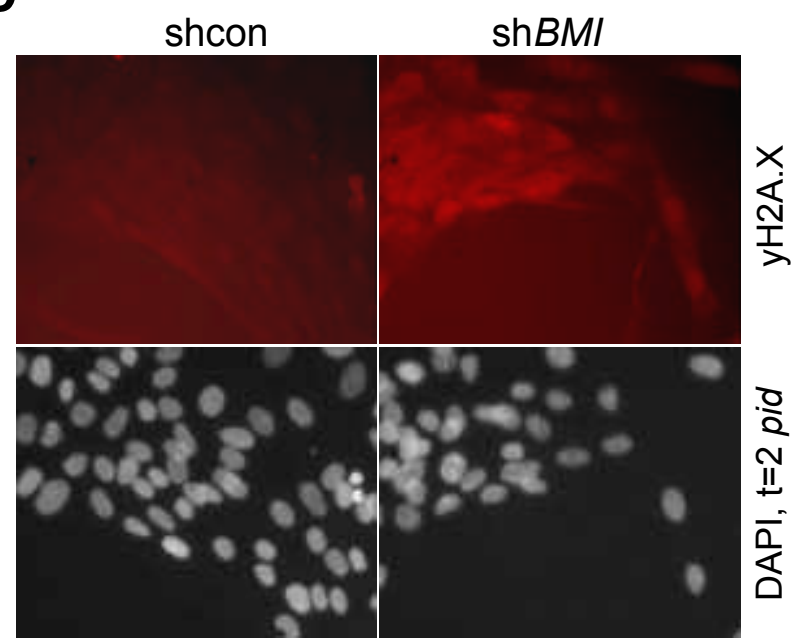

Figure 5.3. ?H2Ax and BrdU staining in $\operatorname{ATDC5}(A, B)$ and human primary chondrocytes (D). 

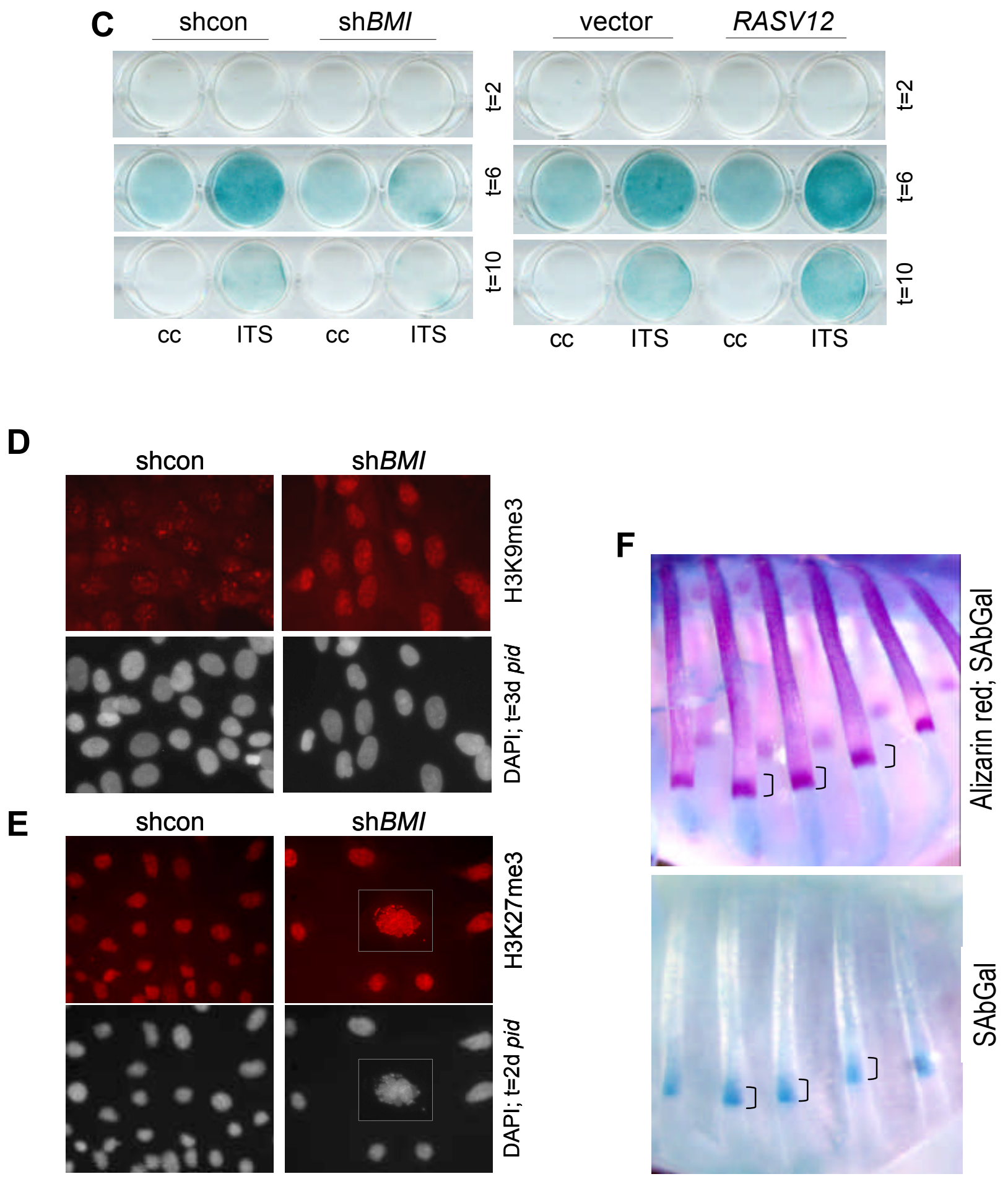

Fig. 5.4 D,E Abnormal staining of histone modifications

Fig. 5.4 C, F Senescence staining ATDC5 cells and ribcage of wildtype mice 

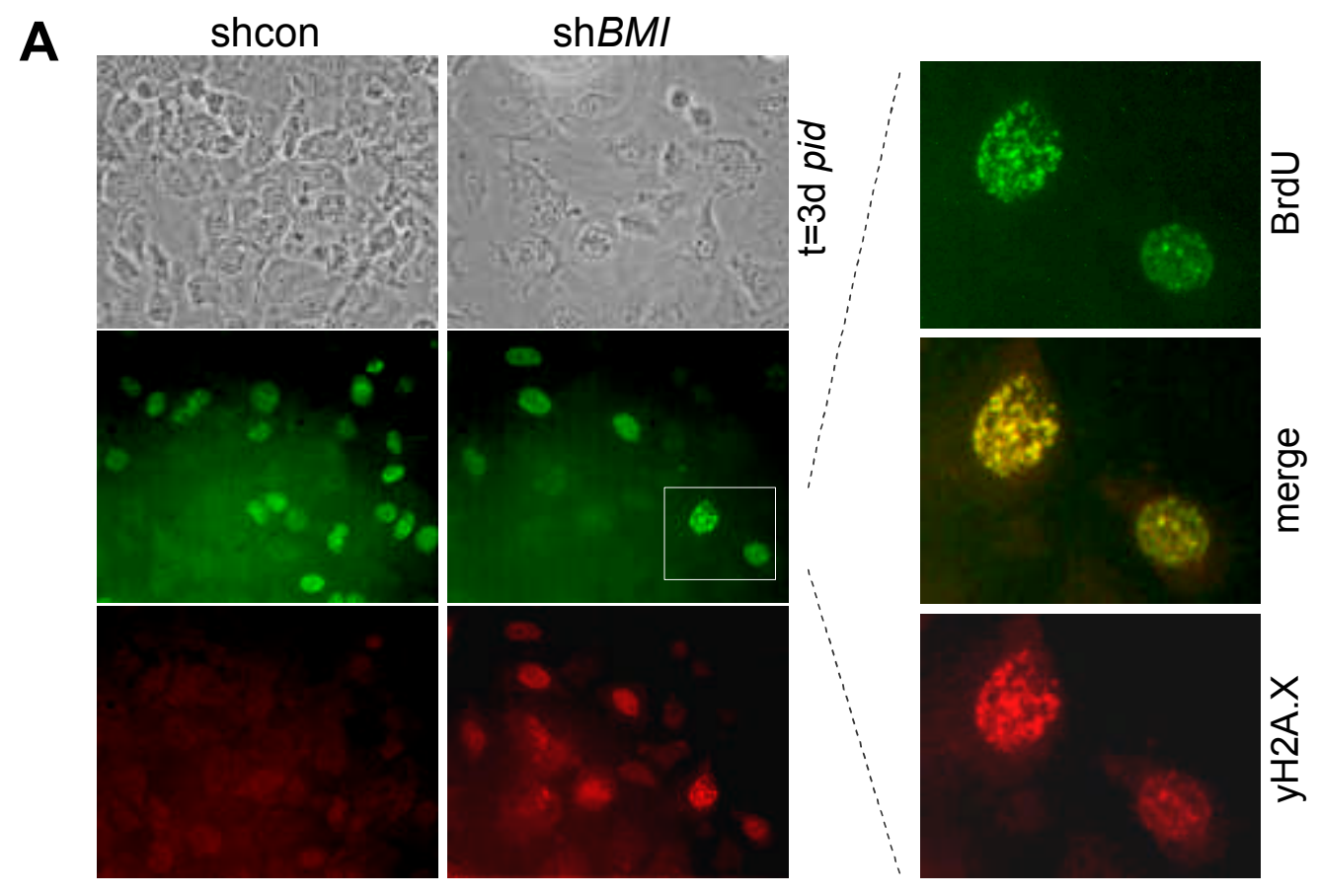

B
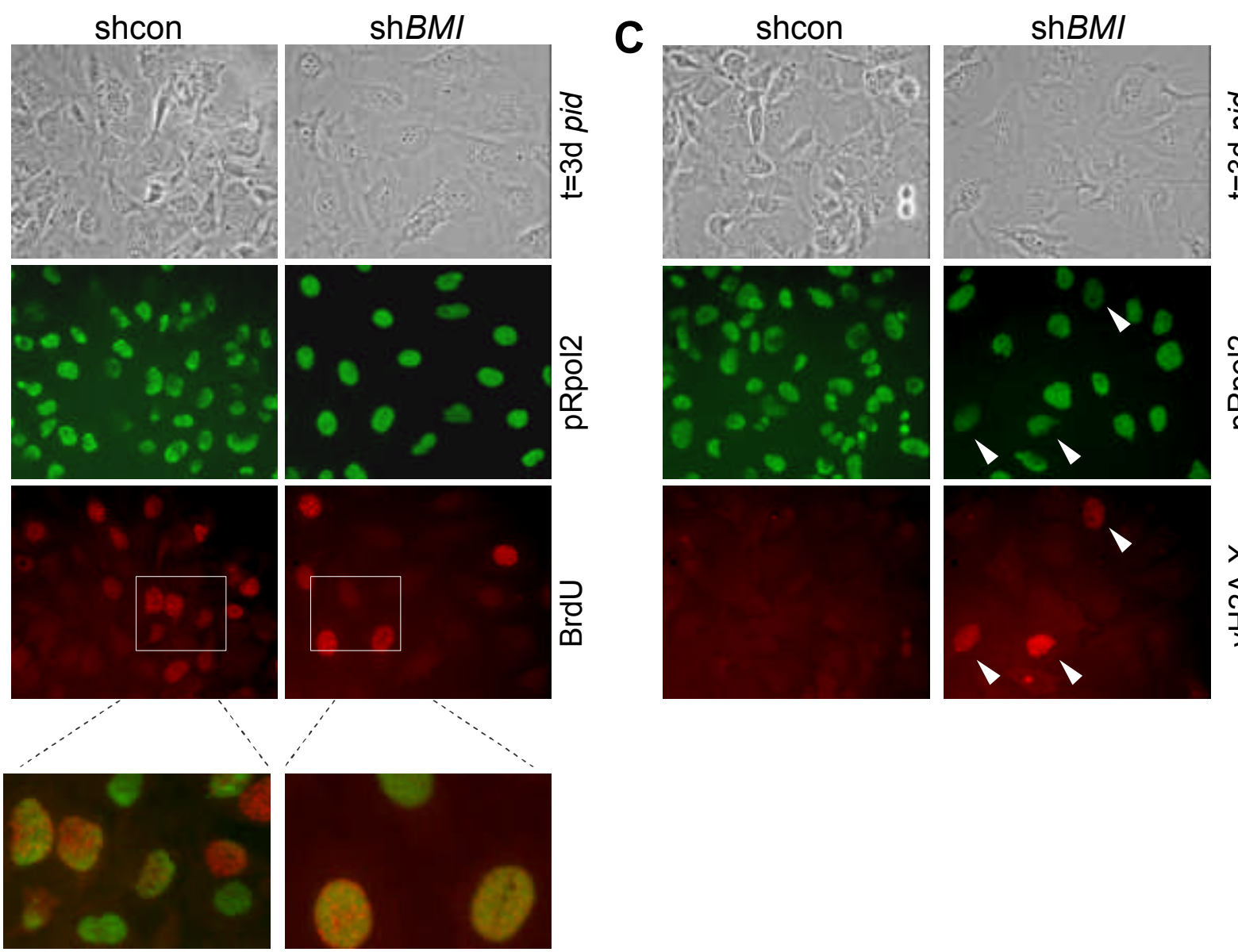

$\frac{7}{2}$
0
II
11
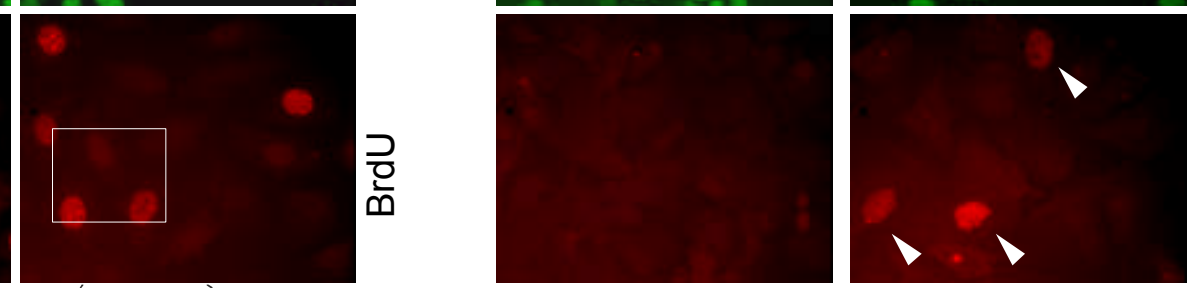

$\frac{N}{\frac{0}{0}}$

Figure 5.6. Accumulation of DNA damage at sites of deregulated replication and transcription. 
A

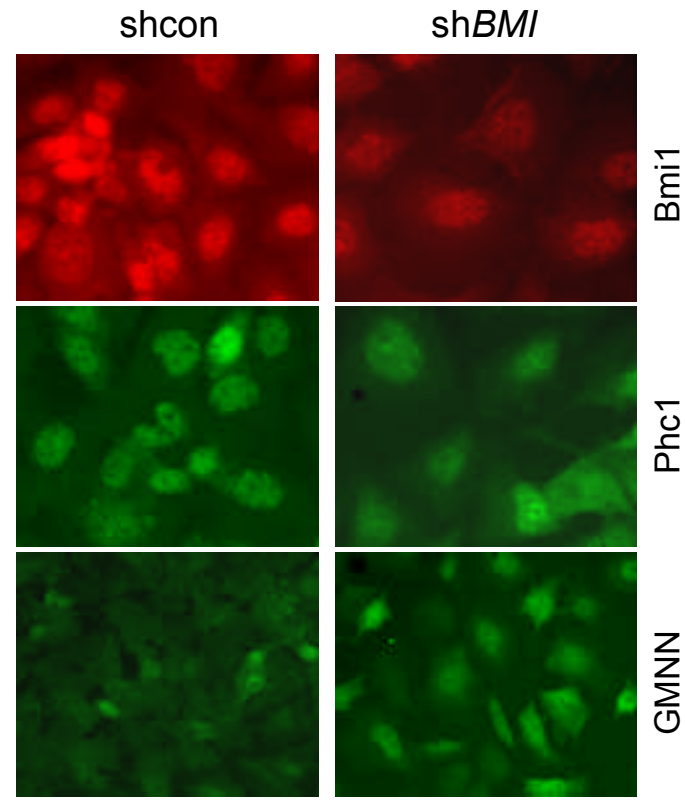

B
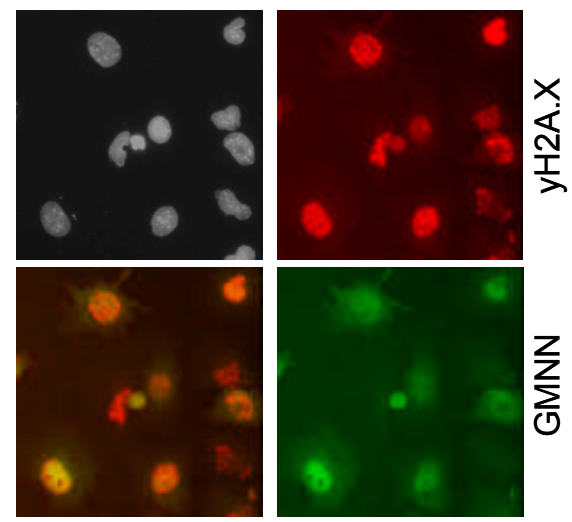

Figure 5.7. IF analysis of Bmi1, Phc1, Geminin and ?H2Ax in ATDC5 shControl and shBmi1.
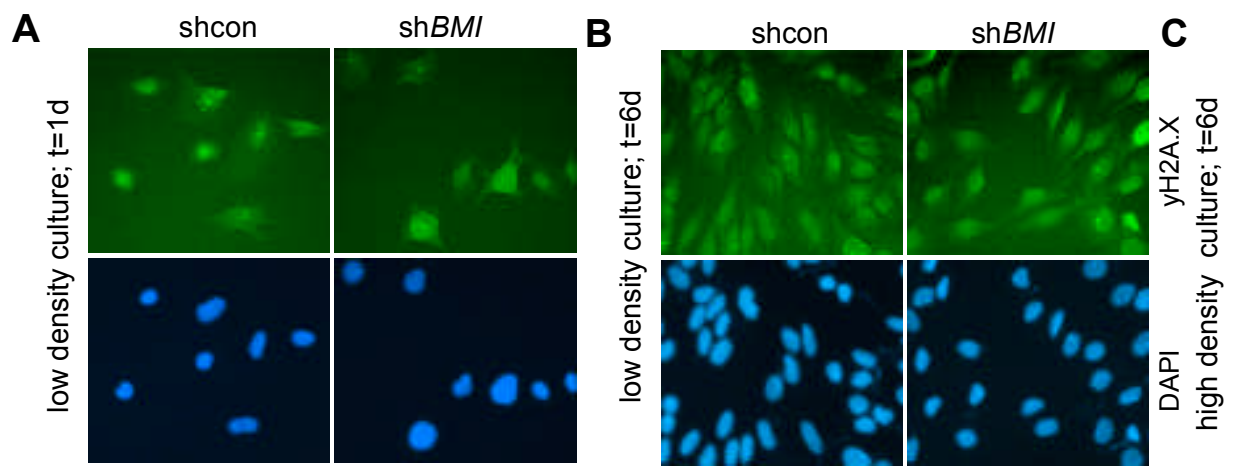

shcon shBMI

Figure 5.S3. Accumulation of DNA damage in shBmi1 cells.

A

shcon

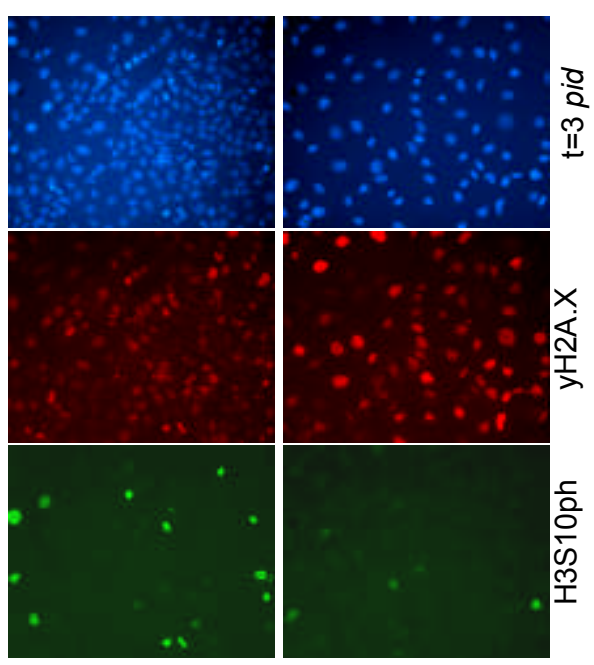

B

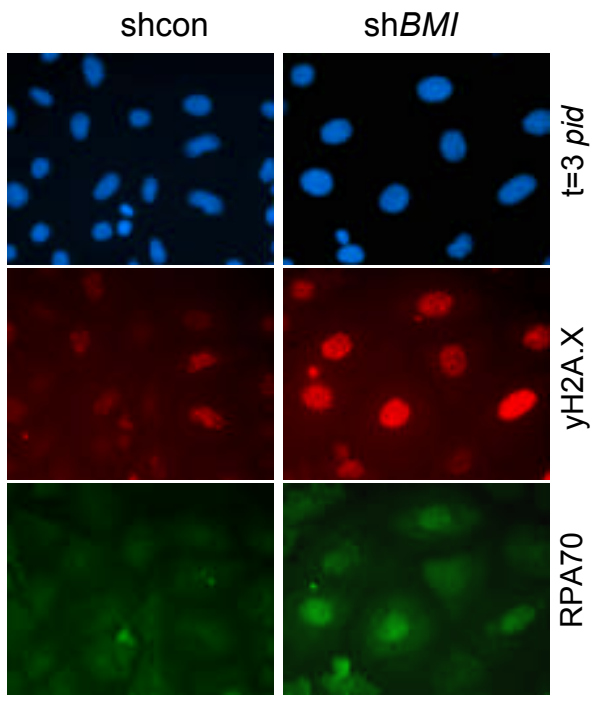

Figure 5.S4. Bmi1-LOF results in DNA damage responses and a proliferative arrest 
C

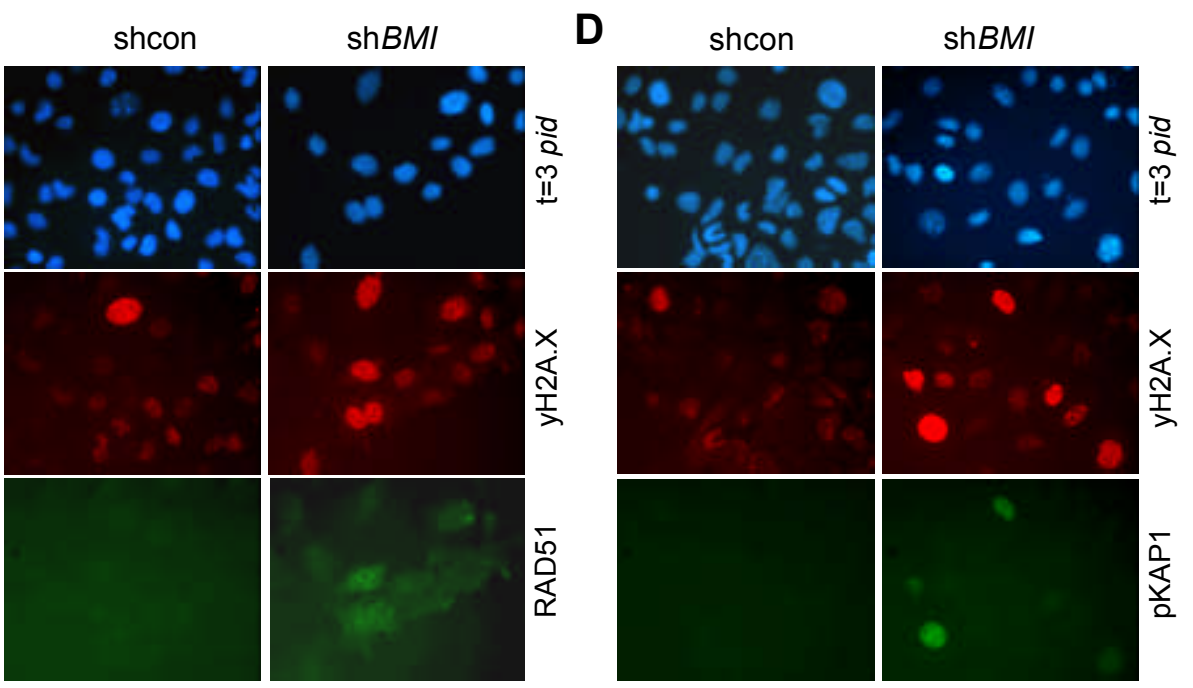

Figure 5.S4 continued. Bmi1-LOF results in DNA damage responses and a proliferative arrest

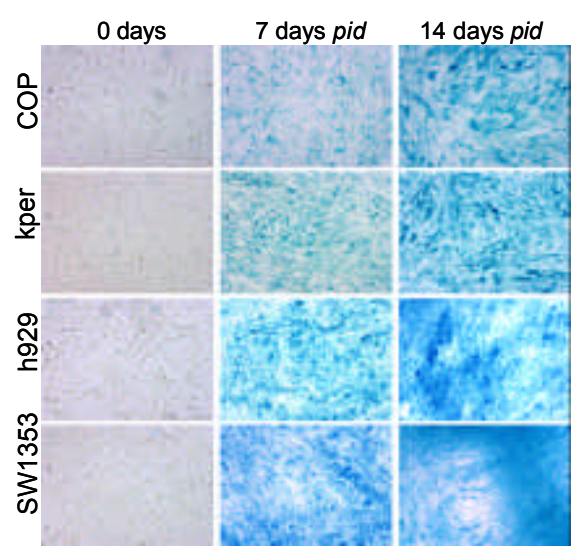

Fig. 5.S5D. Senescence during chondrogenesis
A

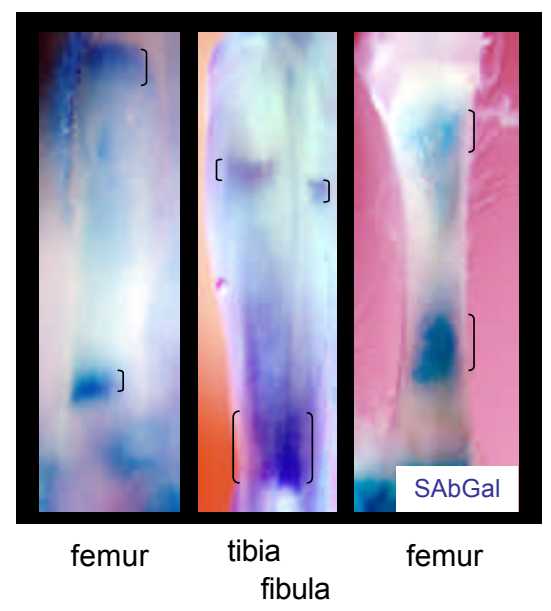

B

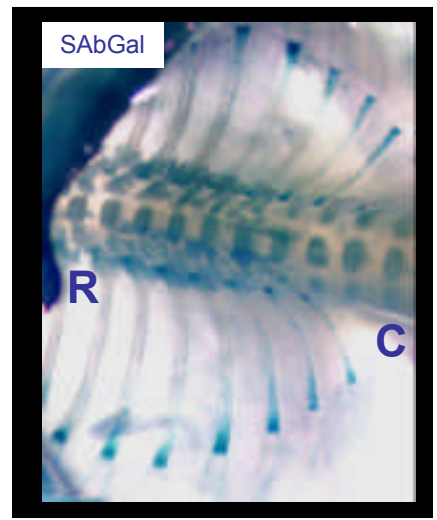

rib cage/spinal column ventral view

Fig. 5.S6 A,B Senescence staining on longitudinal bones ribcage/spinal column of wild type mice 
B

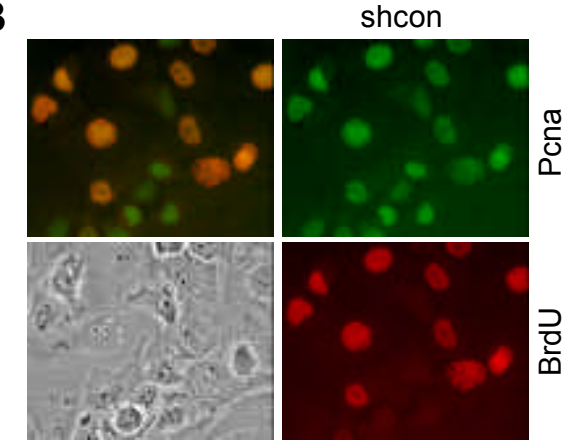

C
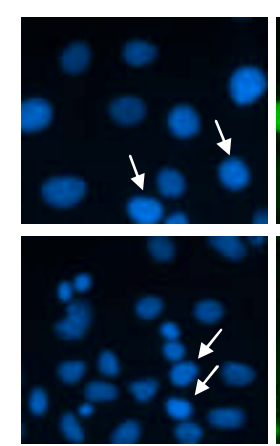

shcon
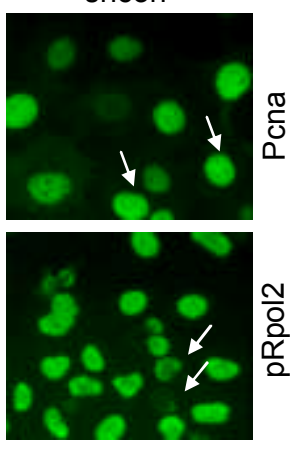

$\operatorname{sh} B M I$

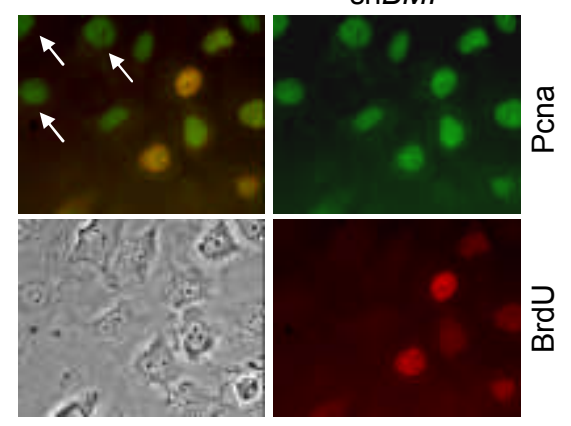

$\operatorname{sh} B M I$
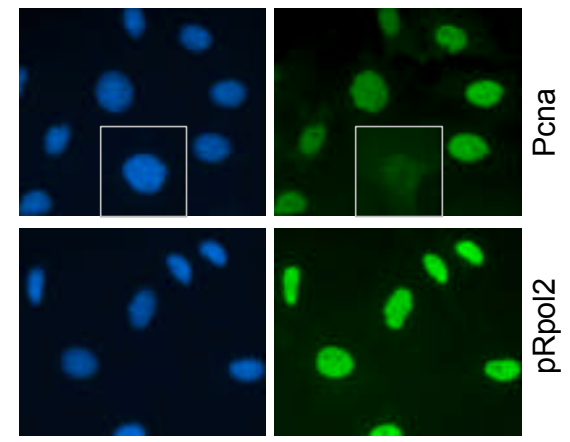

Fig 5.S8. IF staining for PCNA BrdU and pRPOL2 in ATDC5 shControl and shBmi1 at 2d pid.

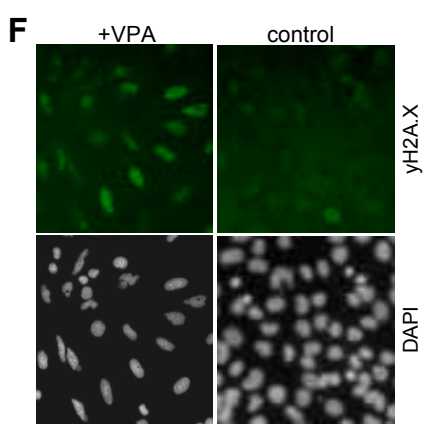

$\mathrm{t}=2$ pid (days)

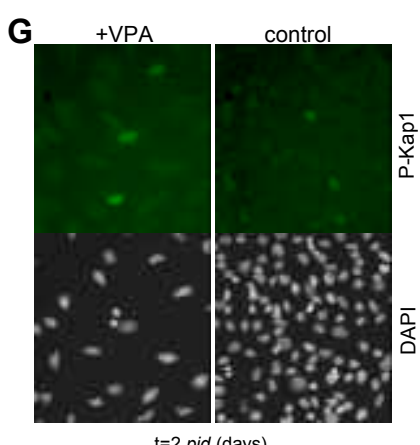

$\mathrm{t}=2$ pid (days)

Fig. 6.2. VPA induces DNA damage responses

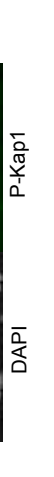

D

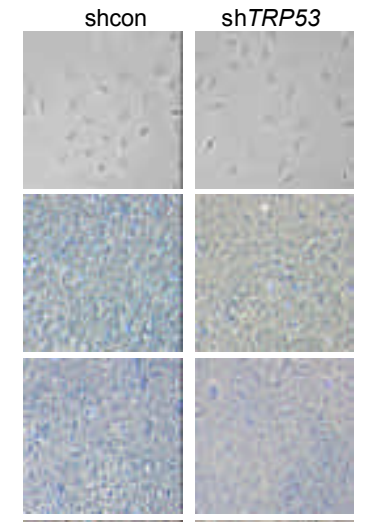

II

$\sum_{\varepsilon}$
$\vdots$
0

$\sum_{\substack{\mathrm{m} \\ 0}}^{1}$

ì
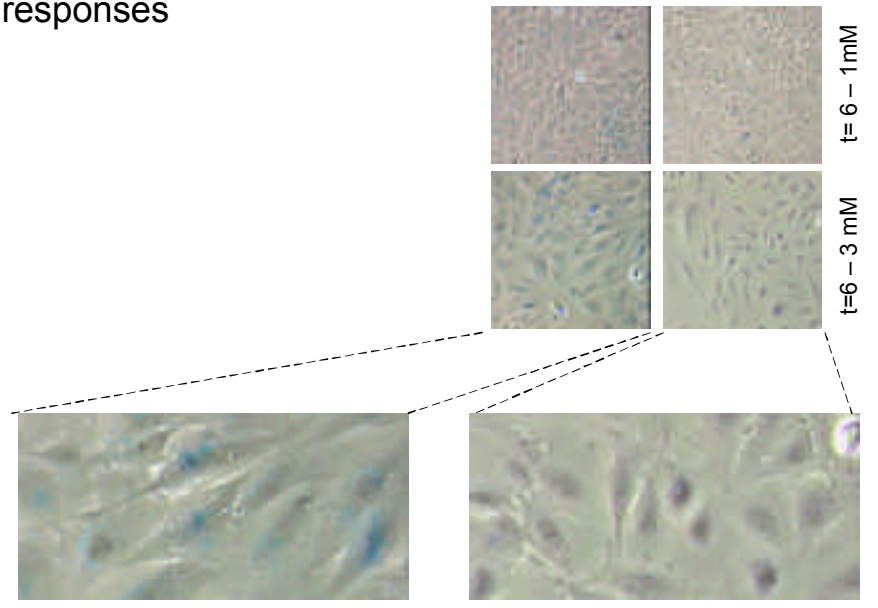

Fig. 6.3. VPA induces senescence 